\title{
Healthcare-seeking behaviour for sexually transmitted infection testing in New Zealand: A mixed methods study
}

by

Hayley Joanna Denison

\author{
A thesis \\ submitted to the Victoria University of Wellington \\ in fulfilment of the requirements for the degree of \\ Doctor of Philosophy \\ Victoria University of Wellington \\ Te Whare Wānanga o te Ūpoko o te Ika a Māui \\ 2017
}







\section{Abstract}

Sexually transmitted infections (STIs) are a global public health problem. Sequelae for infected individuals can be serious and STIs impose a substantial financial burden on healthcare systems. Duration of infection is one factor influencing transmission rates, and is modifiable through secondary prevention methods, namely 'test and treat'. For this approach to be effective, at-risk individuals must choose to present for testing. New Zealand provides a useful case-study to investigate healthcare-seeking behaviour for STI testing, as incidence rates of common STIs are especially high.

The aims of this thesis were to quantify healthcare-seeking behaviour for STI symptoms and assess the risk of transmission in this period, to identify the barriers to STI testing, to understand the personal drivers for getting an STI test, to examine how STI knowledge is associated with testing behaviour, and finally, to collate and critically evaluate the published evidence regarding the incidence of a lesser known sequela of STI, reactive arthritis. This thesis took a mixed method approach, employing both qualitative and quantitative methods to address the research aims.

The results showed that delays in healthcare-seeking for STI symptoms were common among patients attending an inner-city Sexual Health Clinic (SHC). Almost half of people with symptoms waited longer than seven days to seek healthcare, although there were no identified predictors of delayed healthcare-seeking. Around a third of people with symptoms continued to have sex after they first thought they may need to seek healthcare. Among these individuals, infrequent condom use was reported more by those who had sex with existing sexual partners than by those who had sex with new partners. Having sex while symptomatic was statistically significantly associated with delaying seeking healthcare for more than seven days (odds ratio $(\mathrm{OR})=3.25,95 \%$ CI $1.225-8.623, p=0.018$ ).

Analysis of qualitative interview data revealed three types of barriers to testing. These were personal (underestimating risk, perceiving STIs as not serious, fear of invasive procedure, self-consciousness in genital examination and being too busy), structural (financial cost of test and clinician attributes and attitude) and social (concern of being stigmatised). 
This work also revealed several drivers for testing including crisis, partners, clinicians, routines, and previous knowledge. Knowledge of the incidence, asymptomatic nature and sequelae of STIs featured prominently in the explanations of those who undertook routine testing. However, at the same time, many of the participants felt they did not have a good knowledge base and that their school-based sex education had been lacking.

STI knowledge was investigated further using quantitative methodology. Levels of STI knowledge were generally good and did not differ between a Student Health Service population and an SHC population. Individuals who had tested before had significantly better knowledge than those who were attending for testing for the first time $(\mathrm{U}=10089.500, \mathrm{Z}=$ 4.684, $p<0.001)$. In addition, total knowledge score was an independent predictor of having had a previous test $(\mathrm{OR}=1.436,95 \%$ CI 1.217-1.694, $p<0.001)$.

Reactive arthritis can be triggered by STI, thus STI screening patients who present with reactive arthritis has the potential to identify undiagnosed infection. This thesis provides the first assessment of the international literature regarding the incidence of reactive arthritis after STI. The systematic review found only three published studies which had prospectively examined the incidence of reactive arthritis after STI. The studies reported an incidence of reactive arthritis after STI of $3.0 \%$ to $8.1 \%$ and were found to be of low to moderate quality.

In conclusion, this thesis provides healthcare service providers, policy makers and clinicians with data to inform practice and public health interventions aimed at improving healthcareseeking behaviour for STI testing. It illustrates that delayed healthcare-seeking for STI symptoms is a common behaviour in New Zealand and could potentially be contributing to STI transmission and downstream burden on the health system. This work provides evidence of the drivers of STI testing that can be promoted, and the barriers that need to be removed. Specifically, improving STI knowledge may positively impact on testing rates. Lastly, this research indicates that there is a need for more studies assessing the incidence of reactive arthritis after an STI. 


\section{Acknowledgements}

Three years ago I arrived in Wellington to begin my $\mathrm{PhD}$, knowing very few people. Along the way I have had the privilege of meeting and working with some incredibly smart and talented individuals. This $\mathrm{PhD}$ is a culmination of lots of peoples' hard work and support, for which I am extremely grateful.

Firstly, I would like to thank my PhD supervisor, Elaine Dennison, for her endless support and guidance during this process. Thank you for your belief in me and for always making time for me, despite the distance and time-zone differences. I am in awe of your strength and determination and your ability to get even the most seemingly impossible of tasks done. I couldn't have had a better primary supervisor and I will be forever indebted to you for all that you have done for me. Here's to many more years of research together.

Also thanks to my secondary supervisor, Annemarie Jutel, for introducing me to a new world of research and for challenging me to step out of my comfort zone and think deeper and wider. You took me on as a student from a different discipline without really knowing me, and so I thank you for taking that gamble and for your continued faith in me.

To Rebecca Grainger, you were one of the first academics I met in New Zealand and you have made time for me since the beginning of the project. I am so grateful for your knowledge, advice and guidance, and for being someone who can look at the whole picture and connect all of the dots.

To Collette Bromhead, the Queen of Chlamydia, the Goddess of Gonorrhoea, and the High Priestess of HPV. Thank you for your intellect, your pragmatism, and of course your humour. I have learnt so much from you and consider myself very lucky to have you as a colleague and a friend.

Also thank you to John Miller and Phil Lester for being my doctoral committee and providing valuable input into the design and direction of the study.

I am indebted to the study participants, without whom this research would not have been possible. Thank you especially to the twenty-four individuals who allowed me to interview 
them and who shared their stories and feelings with me. My wish is for this work to be used to make things better for everyone. I am also grateful for the financial support provided by the Victoria Doctoral Scholarship and the Wellington Medical Research Foundation.

Many other individuals have contributed to this work and have been wonderful colleagues and friends to me in the process. Thanks to Jane Kennedy and colleagues at the Cuba Street Sexual Health Clinic for your enthusiasm and support for the project. And to the Student Health Services at Victoria, who put up with my interference in their usual practice for rather longer than either of us had hoped. A big thanks to Hansa Patel for all your help with data entry and Beth Curtis for being a fabulous second reviewer for the systematic review. My thanks also go to Sandra Taylor, for being my go-to person for any problems, and an eternal source of positivity. I am grateful to the VUW library staff for their ability to source almost any publication I requested, you do a sterling job. To Jeroen, Hils and colleagues at CPHR, thank you for befriending a lonely epidemiologist when I really needed it. I look forward to working with you in the next chapter of my career. Thanks also to my fellow PhD students at both VUW and CPHR, for chats, lunches, and the sharing of chocolate, and for your friendship and camaraderie. There are too many of you to name individually, but you know who you are.

I am lucky to have the most wonderful friends, both in the UK and in New Zealand. Debs, my lovely, your unwavering support and encouragement has been a true source of strength for me. Thank you for always being there. Alison, I am so grateful for your friendship, you always make yourself available to chat to me and bring me back to earth, even when you were just about to get married! To my second families: Jan, Paul, Jamie and William, and Lin, Stuart, Helen and Louise, thank you for your endless stream of love and encouragement. Louise, your smile is never far from my mind, we all miss you so much.

Moving to New Zealand was initially quite scary, but has been made so much easier by the wonderful people I have met here. Anita and Aidan (and Solomon), Laura and Tim, Adam and Aga, Ian and Jasmine, Chris and Dave, Tim and Francesca; you may not have realised it, but looking forward to spending evenings and weekends with you has kept me going through long hours at the computer. I'm excited for many more adventures with you all. 
I want to say a huge thank you to my wonderful family, who have made all of this possible. To my mum and dad, who cultivated my interest in science and in the world around me from the very beginning; I am eternally grateful. Your support has enabled me to go for my dreams and take risks, as I know you will always be there to catch me if I fall. You are the most wonderful parents and I love you with all of my heart. To Nicole, my sister and my best friend. Thank you for being the rock that keeps me grounded, and for being able to make me laugh no matter what else is going on. I miss you so, so much. To Iain, my brother-in-law, you don't know how grateful I am for your existence. As well as being a lovely friend, you are an amazing husband to my little sister. Thank you also to both of you for bringing my gorgeous nephew, Cody, into this world. His picture sits on my desk and his smiling face has lightened many a tough day. It breaks my heart not to be near you all, and I thank you for your grace and encouragement in my pursuit of my dreams.

I also want to acknowledge the influence of my grandparents, Brenda and Carl Denison, James Davidson, and my wonderful “Grammy”, Lena Davidson. Always in my heart and my thoughts.

And last, but certainly not least, Mark. I could not have done this without you. Your unwavering support and understanding still amazes me. Thanks for making the past three years so much fun and so full of love, and for putting up with me during the difficult points of the $\mathrm{PhD}$. Thank you also for being a chauffeur, cook and housekeeper during the crazy last few months, and for being the reminder that there is more to life than work. If there's one thing this $\mathrm{PhD}$ has given me, it is you, and for that I am deeply thankful. 


\section{Statement of authorship}

I hearby declare that this thesis is my own work and that all sources quoted, paraphrased or otherwise referred to, have been properly acknowledged in the bibliography.

Chapters 4, 5 and 7 of this thesis have been written as individual research papers, and consequently there is some repetition in the introduction and methods sections of those chapters (particularly in Chapters 4 and 5). They have also been written in first person plural. Chapters 4 and 5 have been submitted to international journals and are under review at the time of thesis submission. Chapter 7 has been published by Clinical Rheumatology and is presented in published form in this thesis with only the necessary formatting changes made. In all cases, the manuscripts were written by myself.

The submitted manuscripts from Chapters 4 and 5 include my $\mathrm{PhD}$ supervisors as authors, who contributed to this work by assisting with the design of the project, the interpretation of findings, and the editing of final versions of the papers. The published manuscript (Chapter 7) also includes my PhD supervisors for the same reasons, and two external colleagues who acted as reviewers for the systematic review. Chapters 3 and 6 are not yet submitted for publication but are in the process of being turned into manuscripts. When submitted, these manuscripts will have my PhD supervisors as authors along with a statistician, Lisa Woods, who offered advice with regards to the statistical analysis.

While assisted and supported by my co-authors, my contribution was the greatest. I formulated the concept for the thesis and each individual chapter, designed the studies, prepared the ethics applications, coordinated recruitment and data collection, carried out all of the data cleaning and analysis, and prepared the chapters. 


\section{Table of contents}

Abstract

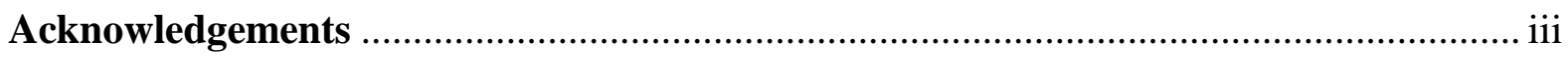

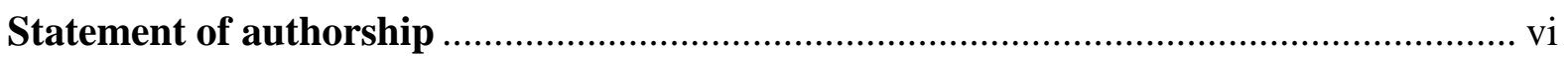

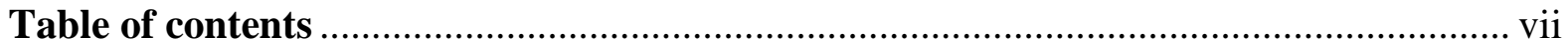

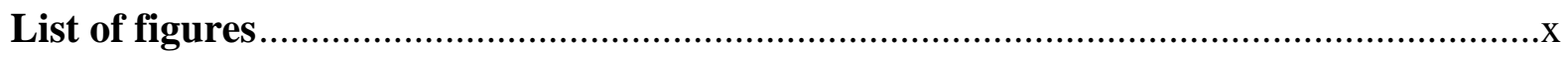

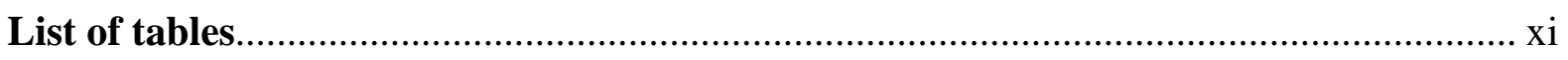

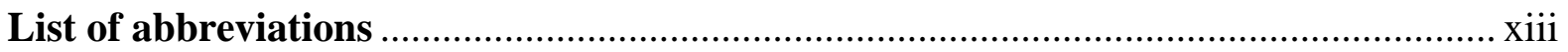

Chapter 1 Introduction, aims and structure of the thesis ..............................................

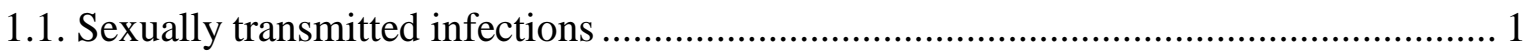

1.2. Sexually transmitted infection prevention............................................................... 3

1.3. New Zealand as a research context …………………........................................... 5

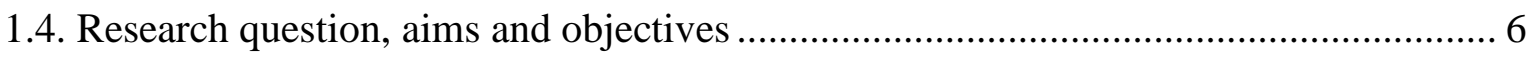

1.5. Explanation of research method approach .............................................................. 7

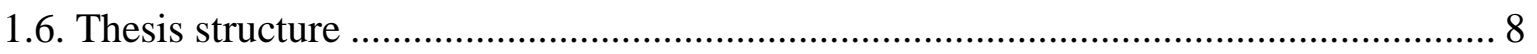

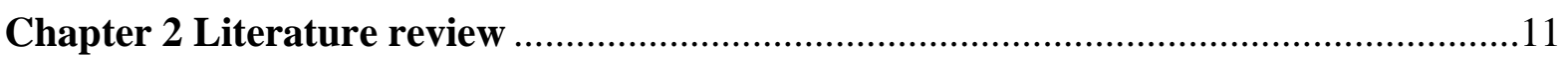

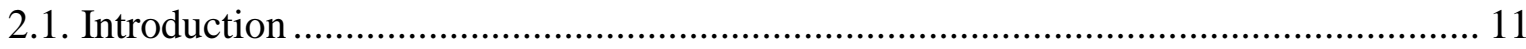

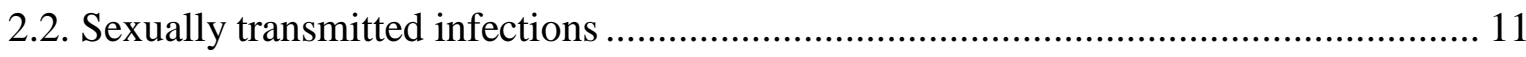

2.3. Burden of disease and factors that increase STI risk .................................................. 29

2.4. Factors that influence the reproductive rate of infection.............................................. 48

2.5. Healthcare-seeking behaviour for STI testing and the factors that may influence it ... 53

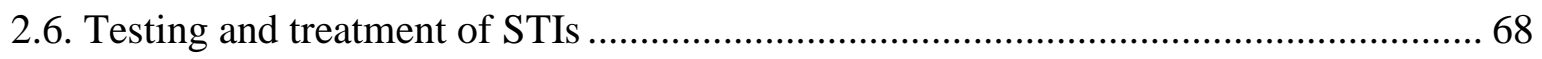

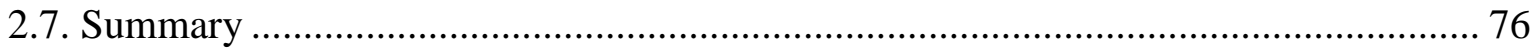

Chapter 3 Healthcare-seeking behaviour of people with sexually transmitted infection symptoms attending an urban Sexual Health Clinic ........................................................79

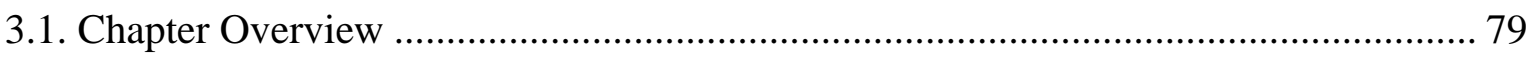

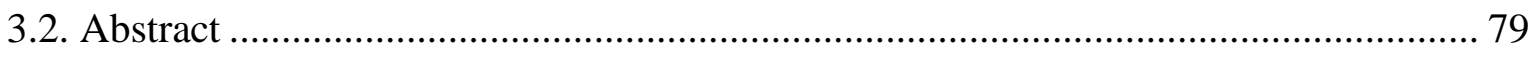

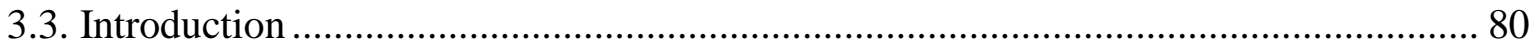

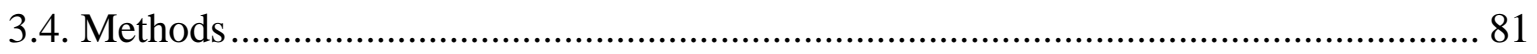




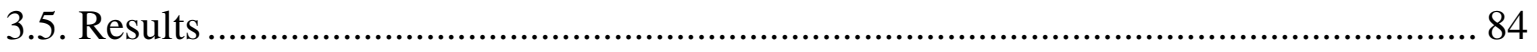

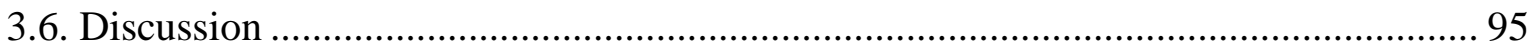

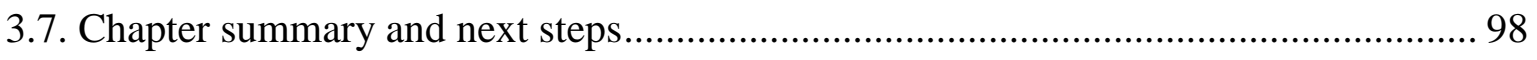

Chapter 4 Barriers to sexually transmitted infection testing in New Zealand: a

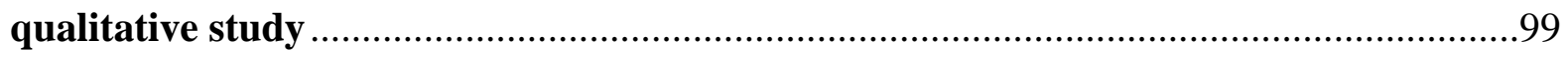

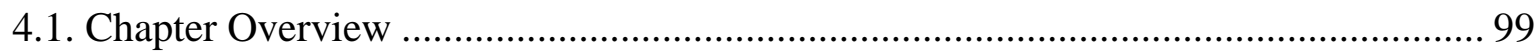

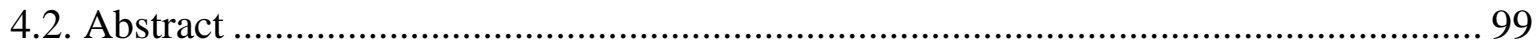

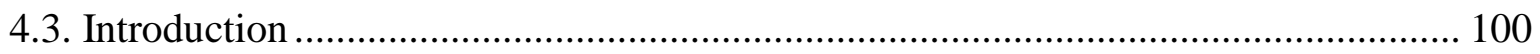

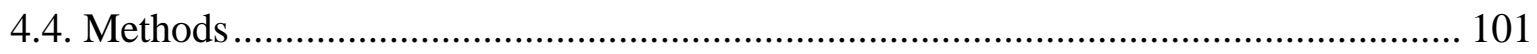

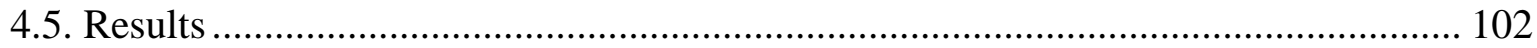

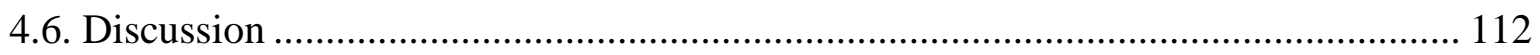

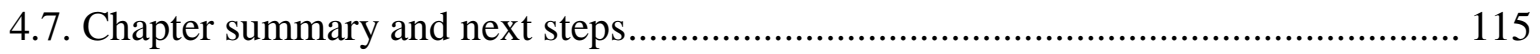

Chapter 5 "It's like brushing your teeth... only every few months": University students'

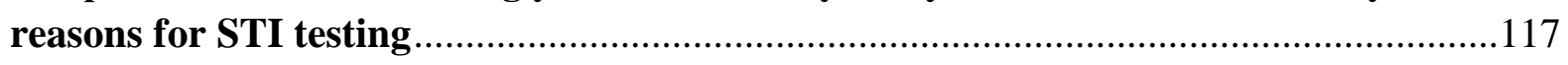

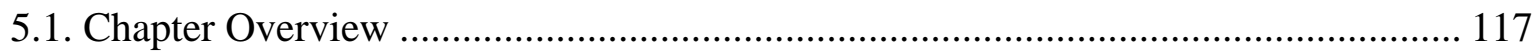

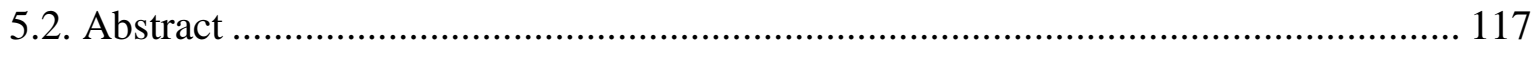

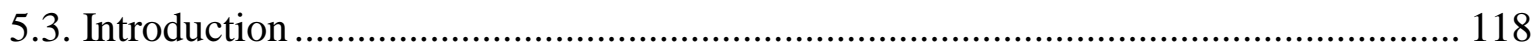

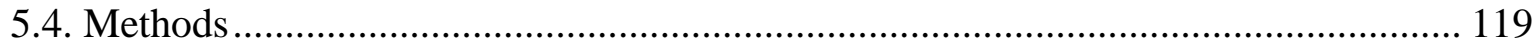

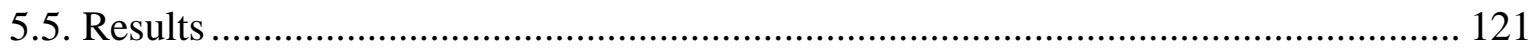

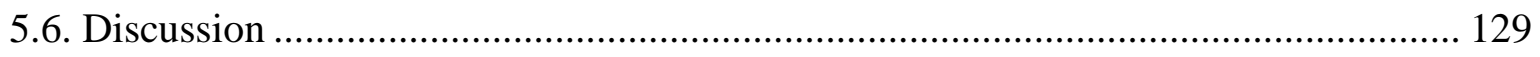

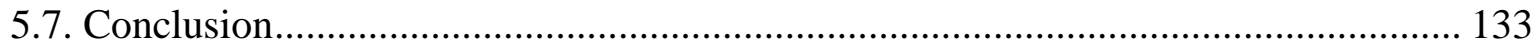

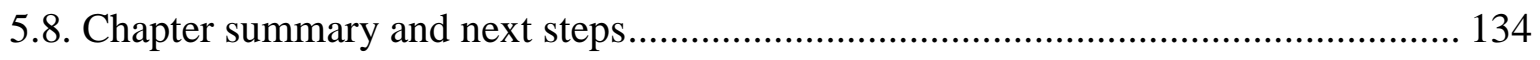

Chapter 6 STI knowledge and its association with STI testing behaviour .....................135

6.1. Chapter Overview …………………………………........................................ 135

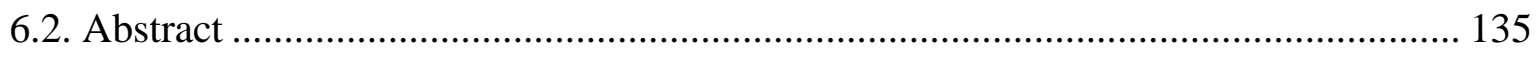

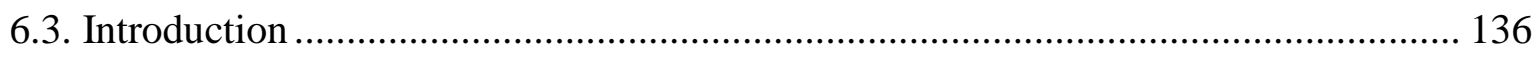

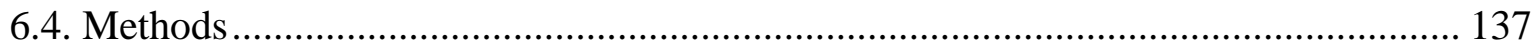

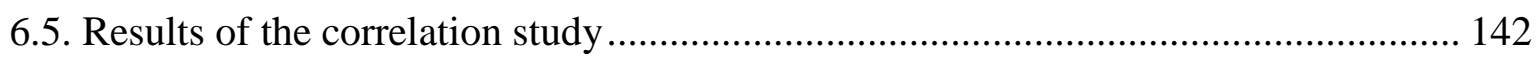

6.6. Results of the main study …………….............................................................. 143

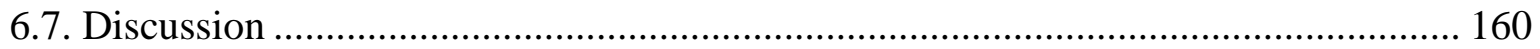

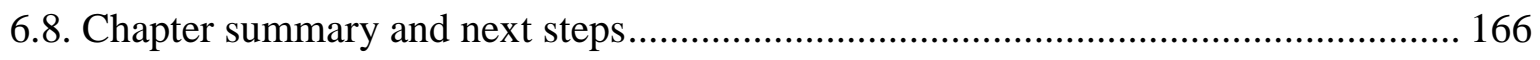


Chapter 7 The incidence of sexually acquired reactive arthritis: a systematic literature review

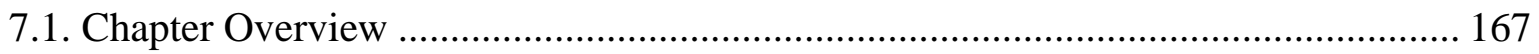

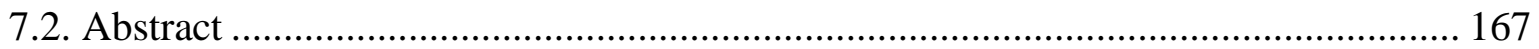

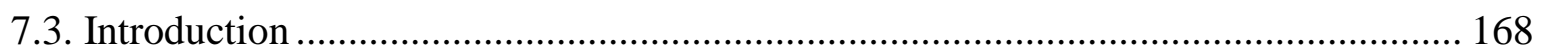

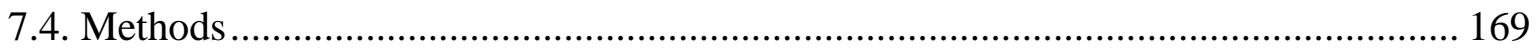

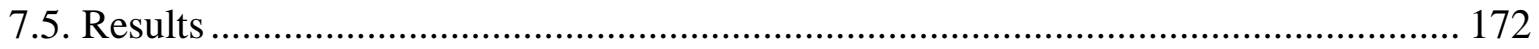

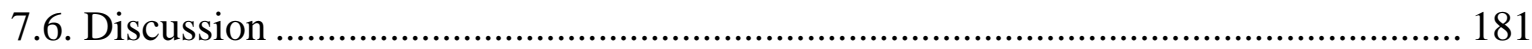

7.7. Chapter summary and next steps....................................................................... 185

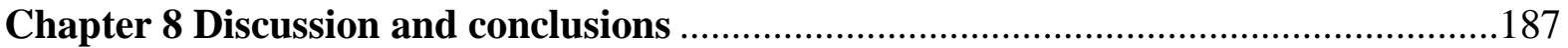

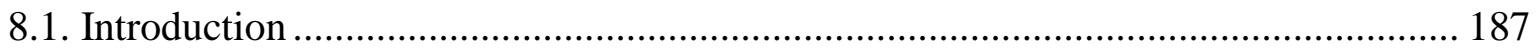

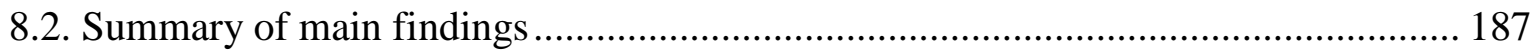

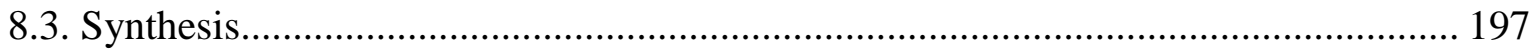

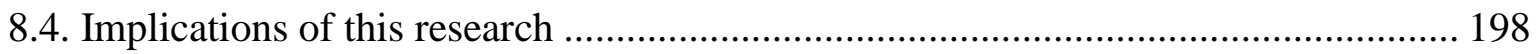

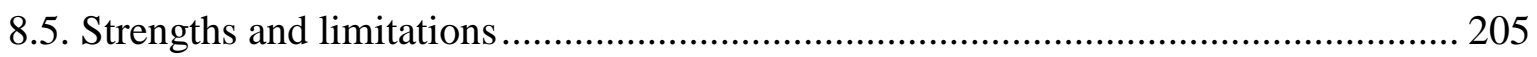

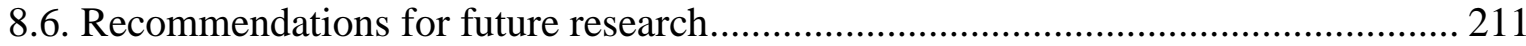

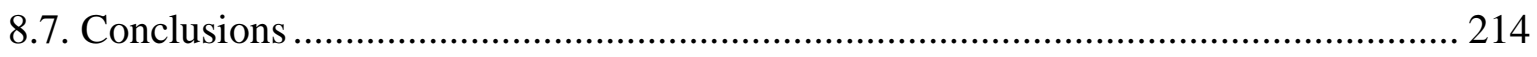

Appendix A. Questionnaire and detailed explanation of questionnaire items .......... 217

Appendix B. Developing a recruitment methodology: Results of a focus group study 225

Appendix C. Supplementary tables for Chapter 6 ……………………………..... 239

Appendix D. Ethical approval confirmations ………………………………........... 243 


\section{List of figures}

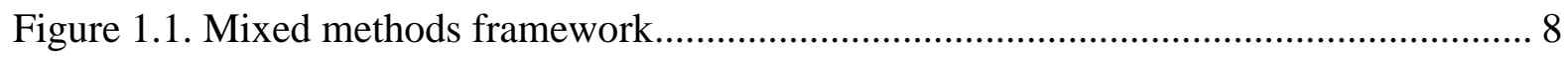

Figure 2.1. Chlamydia (blue) replicating inside vesicle within a cell ............................... 14

Figure 2.2. Neisseria gonorrhoeae bacteria contained within phagocytic leukocytes ........... 15

Figure 2.3. Lesions of secondary syphilis rash on a forearm.......................................... 19

Figure 2.4. Human immunodeficiency viruses infecting a cell ....................................... 20

Figure 2.5. National chlamydia rate per 100,000 population over time …........................... 33

Figure 2.6. National gonorrhoea rate per 100,000 population over time............................. 33

Figure 2.7. Rates of chlamydia per 100,000 population for four developed countries in 2014

Figure 2.8. Rates of gonorrhoea per 100,000 population for four developed countries in 2014

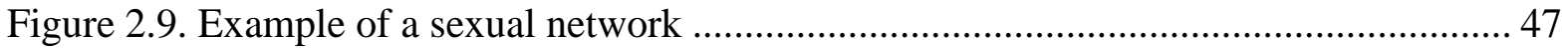

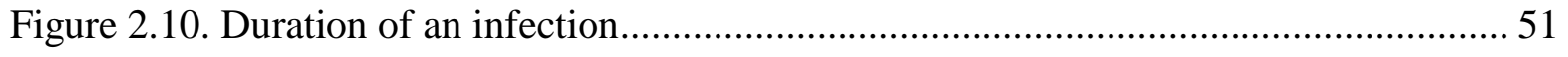

Figure 3.1. Histogram showing the distribution of delay behaviour ................................. 87

Figure 6.1. Histogram showing frequencies of total knowledge scores for both samples

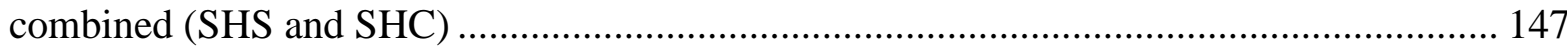

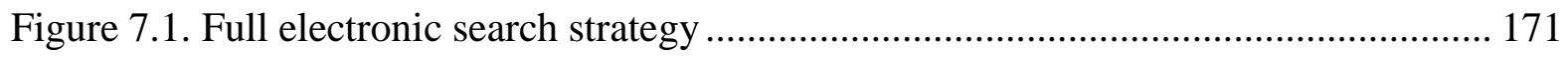

Figure 7.2. Flow diagram depicting the identification and selection of relevant papers ..... 174

Figure 7.3. Screening questionnaire suggested for use in studies of the incidence of SARA, adapted from Townes et al. 2008 . 


\section{List of tables}

Table 2.1. Common sexually transmitted infections 13

Table 2.2. Estimated global prevalence of four STIs (95\% uncertainty interval shown in

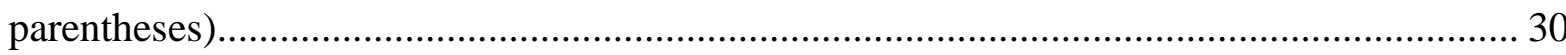

Table 2.3. Published studies assessing time to presentation for STI symptoms................... 57

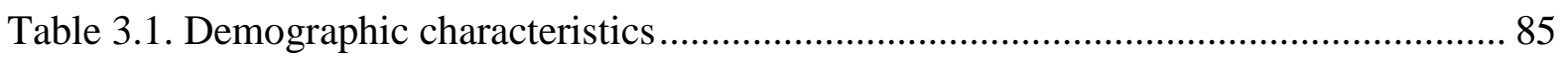

Table 3.2. Reasons for attending for current test, previous testing behaviour and previous

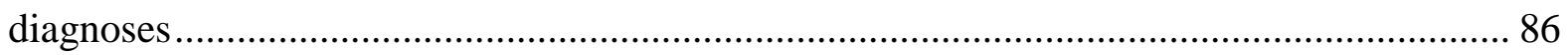

Table 3.3. Healthcare-seeking and sexual behaviour of symptomatic respondents $(n=91) . .88$

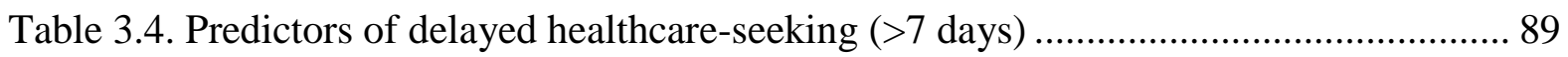

Table 3.5. Sexual behaviour since symptom onset and contacting healthcare services ........ 91

Table 3.6. Predictors of positive diagnosis at current visit ............................................ 93

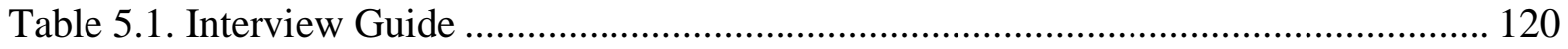

Table 5.2. Demographic profile of participants ......................................................... 122

Table 6.1. Mean, median, minimum and maximum values for the STD-K questionnaire for each scoring level of the 7-item knowledge questionnaire.

Table 6.2. Comparison of demographic characteristics and testing history of those attending

Student Health Services and those attending the Sexual Health Clinic 145

Table 6.3. Comparison of sexual behaviours of those attending Student Health Services and those attending the Sexual Health Clinic ................................................................. 146

Table 6.4. Comparison of correct response to individual knowledge questions between SHS

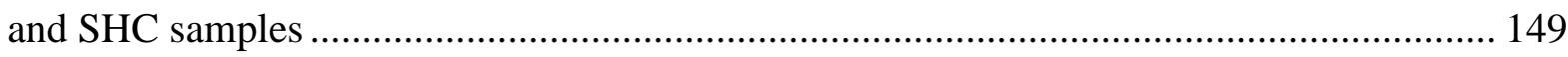

Table 6.5. Difference in knowledge by sociodemographic and sexual behaviour characteristics. 151

Table 6.6. The effect of sociodemographic variables on total knowledge score for the whole sample $(n=546)$ 153

Table 6.7. Difference in knowledge by testing history..... 154

Table 6.8. Predictors of having had a previous STI test $(n=322)$. 155

Table 6.9. Relationship between self-assessed knowledge and measured knowledge 156

Table 6.10. The effect of campaign awareness on STI knowledge with adjustment for demographic characteristics $(n=528)$. 158 
Table 6.11. The effect of campaign awareness on previous STI testing after adjustment for

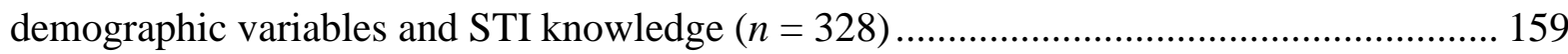

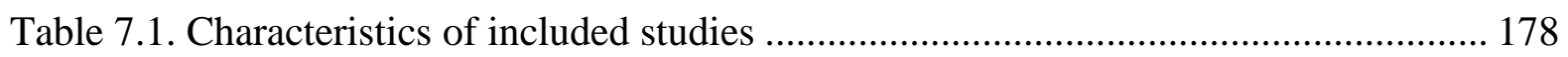

Table 7.2. ReA diagnosis methodology of included studies............................................... 179

Table 7.3. Risk of bias assessment using RTI Item Bank tool ............................................ 180

\section{Tables in appendices}

Table AB.1. Factors which may influence taking part in a questionnaire about STIs ......... 230

Table AB.2. Personal motivators for take part in research studies ........................................ 232

Table AB.3. Confidentiality considerations................................................................... 233

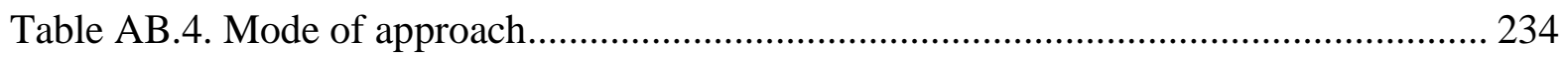

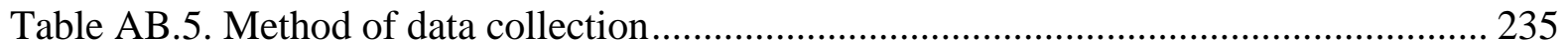

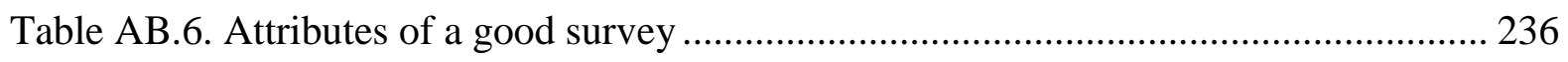

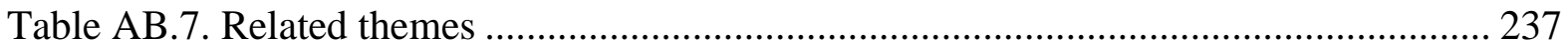

Table AC.1. Difference in knowledge by sociodemographic and sexual behaviour

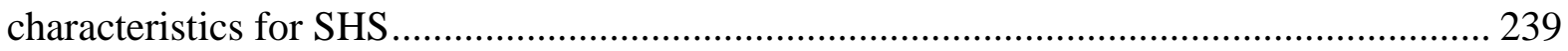

Table AC.2. Difference in knowledge by sociodemographic and sexual behaviour

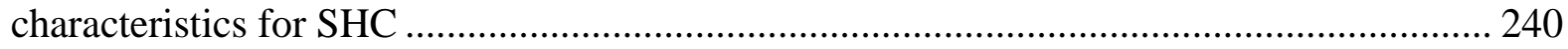

Table AC.3. The effect of sociodemographic variables on total knowledge score for the SHS

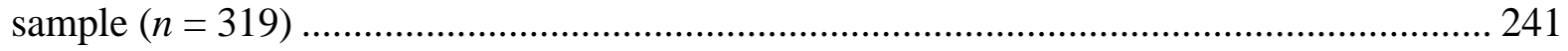

Table AC.4. The effect of sociodemographic variables on total knowledge score for the SHC

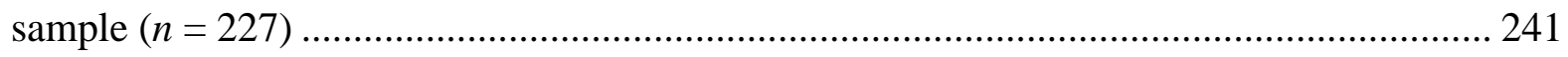

Table AC.5. Ordinal regression of total knowledge score with sexual behaviour characteristics added into the model, for both samples combined $(n=331)$. 


\section{List of abbreviations}

\begin{tabular}{ll} 
AIDS & acquired immune deficiency syndrome \\
ART & antiretroviral therapy \\
AUDIT-C & Alcohol Use Disorders Identification Test - Consumption questions \\
BV & bacterial vaginosis \\
CDC & Centers for Disease Control and Prevention \\
CI & confidence interval \\
CME & continuing medical education \\
DHB & district health board \\
DNA & deoxyribonucleic acid \\
ELISA & enzyme-linked immunosorbent assay \\
ESR & Institute of Environmental Science and Research Ltd \\
ESSG & European Spondyloarthropathy Study Group \\
EVD & Ebola virus disease \\
FET & Fisher's exact test \\
FHC & Fitz-Hugh-Curtis syndrome \\
FPC & Family Planning Clinic \\
GP & general practitioner \\
GUM & genitourinary medicine \\
HIV & human immunodeficiency virus \\
HLA-B27 & human leukocyte antigen B27 \\
HPV & human papillomavirus \\
HrHPV & high risk human papillomavirus \\
HSV & herpes simplex virus \\
LGBTIQ & lesbian, gay, bisexual, transgender, intersex and questioning \\
\hline
\end{tabular}




$\begin{array}{ll}\text { MeSH } & \text { Medical Subject Headings } \\ \text { MSM } & \text { men who have sex with men } \\ \text { MSW } & \text { men who have sex with women } \\ \text { NAAT } & \text { nucleic acid amplification test } \\ \text { NCSP } & \text { National Chlamydia Screening Programme } \\ \text { NZAF } & \text { New Zealand AIDS Foundation } \\ \text { Natsal } & \text { National Surveys of Sexual Attitudes and Lifestyles } \\ \text { OR } & \text { odds ratio } \\ \text { PID } & \text { pelvic inflammatory disease } \\ \text { PPV } & \text { positive predictive value } \\ \text { R0 } & \text { basic reproductive number } \\ \text { RCT } & \text { randomised controlled trial } \\ \text { ReA } & \text { reactive arthritis } \\ \text { RNA } & \text { ribonucleic acid } \\ \text { SARA } & \text { sexually acquired reactive arthritis } \\ \text { SD } & \text { standard deviation } \\ \text { SHC } & \text { sexual health clinic } \\ \text { SHS } & \text { Student Health Services } \\ \text { STD-KQ } & \text { Sexually Transmitted Diseases Knowledge Questionnaire } \\ \text { STI } & \text { sexually transmitted infection } \\ \text { UK } & \text { United Kingdom } \\ \text { USA } & \text { United States of America } \\ \text { WHO } & \text { World Health Organization } \\ \text { WSM } & \text { Women who have sex with men } \\ \text { Women who have sex with women } \\ \text { WSW }\end{array}$




\section{Chapter 1 Introduction, aims and structure of the thesis}

\subsection{Sexually transmitted infections}

Sexually transmitted infections (STIs) cause significant morbidity and mortality worldwide. STIs can lead to serious sequelae including pelvic inflammatory disease (PID), infertility, adverse pregnancy outcomes, rheumatological complications, cancer, organ damage and death (Singh and Romanowski, 1999, zur Hausen, 1999, Bosch et al., 2002, Idahl et al., 2004, Hamdulay et al., 2006, Haggerty et al., 2010, Poorolajal et al., 2016). These health outcomes present a significant financial burden to healthcare systems as well as considerable physical and emotional suffering for infected individuals (Melville et al., 2003, Piercy, 2006a).

STIs have afflicted humans for as long as records exist; descriptions of gonorrhoea and syphilis can be found in ancient texts from around the world (Oriel, 1994, Tampa et al., 2014, Morgan and Decker, 2016). The modern era has seen the rise of chlamydia and the human immunodeficiency virus (HIV), and there are continually new infections emerging as STIs, a recent example being Zika Virus (Musso and Gubler, 2016). Although progress has been made in terms of treatments, (Cruciani et al., 2014, Pau and George, 2014, Moi et al., 2015), and more recently, vaccine development (Harper et al., 2004, Harper et al., 2006, Cutts et al., 2007), we are a long way from successfully controlling or eradicating these pathogens.

STIs are recognised as a serious public health issue by many international health agencies, research organisations and national governments. For example, The World Health Organization (WHO) has developed three global health sector strategies, endorsed by the sixty-ninth World Health Assembly in May 2016, which provide a framework for action to tackle HIV, viral hepatitis, and STIs over the period 2016 to 2021 (World Health Organization, 2016a). The Guttmacher Institute and The Lancet have recently announced that they are establishing a Commission which aims to define priorities and produce a set of evidence-based recommendations for sexual and reproductive health and rights (Starrs, 
2015). Many individual countries also have their own guidelines and policies regarding STIs and sexual health (European Centre for Disease Prevention and Control, 2014).

Despite the interest and substantive motivations for tackling this issue, incidence rates of STIs continue to be high. The WHO estimates that in 2012 there were 131 million new cases of chlamydia, 78 million of gonorrhoea, 143 million of trichomoniasis, and 6 million of syphilis, all of which are curable infections (Newman et al., 2015). There are a number of reasons for these high incidence rates, including the asymptomatic nature of STIs resulting in people spreading infection unknowingly, and a lack of access to testing and treatment in developing countries. Research into STIs and STI prevention has also been hindered, preventing progress. One reason for this is that STIs are stigmatised infections, associated with deviant behaviour and dubious morality. Their taboo nature has prevented open discussion and coordinated confrontation of the problem. For example, when the first British National Survey of Sexual Attitudes and Lifestyles (Natsal) was planned in 1989, the then British government, led by Margaret Thatcher, vetoed public funding of the study stating that it was too intrusive (Maddox, 1989, Wellings et al., 1990). It is argued that this politicising of sexual health research continues today with the 2016 United Nations Sustainable Development Goals (SDGs) taking a narrow view of sexual and reproductive health and rights, and omitting specific mention of STIs (Starrs, 2015).

A further issue impacting on progress with regards to STIs is the perceived dichotomy between HIV and other STIs which has left STI research with much less funding and attention (Steen et al., 2009). As explained in a recent editorial in The Lancet Infectious Diseases (Sept 2016):

"Over the past few decades, most sexually transmitted infections (STIs) have been viewed as the poor relations of HIV and, more recently, viral hepatitis. STIs have been paid little attention and accorded only peripheral interest because the safe-sex messages related to HIV have been assumed sufficient to keep them under control. However, this lack of focus beyond those directly involved with the field is leading the health-care community perilously close to disaster." 
Another perceived dichotomy that may also have undermined efforts to successfully understand and control STIs is that of research disciplines. Traditionally research methodologies have been split between quantitative and qualitative, and until recently, these have generally been viewed as mutually exclusive approaches in the pursuit of knowledge. Over the past two to three decades these methodologies have been brought together in multi and mixed method studies, adding depth and breadth to our understanding of various public health issues. This is especially pertinent to STI research, as STIs are spread by sexual contact meaning they are firmly located within, and influenced by, social and cultural structures. Therefore, research into STI prevention needs to be aware of and incorporate evaluation of these influences so that resulting knowledge is applicable to the complex realworld setting.

\subsection{Sexually transmitted infection prevention}

STI control strategies can be broadly grouped into two categories, primary and secondary prevention. Although these prevention efforts take different approaches, established epidemiological wisdom suggests that both systems need to be employed in order to successfully bring STI transmission under control. Primary prevention aims to prevent new cases of infection and is the first-line of defence against the spread of STIs. The mainstay of primary prevention is condom promotion and distribution (European Centre for Disease Prevention and Control, 2016). However, condom use is less than adequate in many populations (Reece et al., 2010, Fennell, 2014) and there is evidence of condom-fatigue in recent years, potentially due in part to improved therapies for HIV (Beltzer et al., 2013). Secondary prevention refers to preventing onwards transmission of infection by identifying existing infections and either removing them from the population by curative treatment or managing incurable infections to minimise risk of transmission (European Centre for Disease Prevention and Control, 2016). In order to identify existing infection, individuals at risk of STIs need to present for testing, which can be challenging because STIs do not always produce clinical signs and symptoms. 
Another mode of secondary prevention is screening, either in the form of systematic general population screening or opportunistic recommendation to people attending healthcare services (Low et al., 2016). However, the prevalence of most STIs, other than Chlamydia trachomatis, are too low for screening to be effective (Fifer and Ison, 2014). Even in the case of $C$. trachomatis, the evidence for the effectiveness of screening on transmission or reproductive tract morbidity is far from conclusive (Low et al., 2016). There are also potential harms involved in screening, such as the possibility of causing anxiety in screened individuals (Mills et al., 2006) and the potential of false positives, which is increased in populations in which C. trachomatis is less common (Hocking et al., 2013). Although opportunistic testing is recommended in many countries, there are few which have an organised screening programme. Therefore, encouraging people at risk of STIs to present for testing is a key priority. In order to facilitate this behaviour, we need to have a comprehensive understanding of healthcare-seeking behaviour for STI testing. Particularly important research questions relate to current patterns of behaviour, barriers to testing, motivations for testing, the systems of lay knowledge which affect testing behaviour, and the pathways to care.

Many studies have examined delay behaviour in healthcare-seeking for STI (Leenaars, 1993, Sawyer and Moss, 1993, Pitts et al., 2000, Malek et al., 2013, Mercer et al., 2013a), but far fewer have actually assessed whether this delay behaviour poses a significant transmission risk. Without knowing whether people continue to have sex while delaying seeking healthcare, and what the patterning of partner-choice and condom-usage is, we cannot determine whether this is an important target for intervention. Although there is a large amount of research investigating the barriers to testing, much less has focussed on the reasons for testing (Yeung et al., 2015). Both aspects need to be understood in order to develop methods to improve testing rates. To date, STI knowledge has mainly been assessed in relation to primary prevention strategies such as condom use (Kellock et al., 1999, Diclemente et al., 2002, Burazeri et al., 2004, Mmbaga et al., 2008, Greaves et al., 2009). There have been few studies investigating the association of STI knowledge with healthcare-seeking behaviour, representing an important gap in understanding. 
An alternative trigger to seek healthcare is experiencing symptoms of sequelae, rather than symptoms of the initial infection itself. This provides an opportunity for uncovering undiagnosed infection in people who may not have sought STI testing themselves, but present to healthcare providers via another pathway. To maximise the potential benefit in terms of reduction of transmission, the earlier the infection is diagnosed, the better. One health outcome of STI which often occurs in the first few weeks after infection exposure is reactive arthritis (ReA) (Hamdulay et al., 2006). Ensuring those who present to healthcare providers with symptoms of ReA are subsequently tested for STIs has the potential to remove undiagnosed STI from the population, as well as relieve rheumatological symptoms for the individual. However, what is not known is how many people with STI this sequela affects. To date, there has been no systematic review assessing the incidence of ReA after STI.

\subsection{New Zealand as a research context}

STI incidence in New Zealand is high. The 2014 national rate of diagnosed chlamydia infection was 869 per 100,000 population for women and 375 per 100,000 population for men, which is 1.4 to 1.7 times higher than rates reported from Australia, the USA and the UK (ESR Annual Surveillance Report 2014). However, little is known about healthcare-seeking behaviour for STI in New Zealand. To the author's knowledge, there have been no published studies assessing the length of time a person with symptoms suggestive of an STI waits before seeking healthcare in New Zealand, or about the potential transmission risk in this period. In addition, there have been no published studies investigating the reasons people test or the barriers to testing in the past decade, despite the rates of common STIs being consistently high. There also seems to have been no studies assessing whether STI knowledge is associated with STI testing in a New Zealand population. As a result of this lack of New Zealand-specific data, our current understanding of healthcare-seeking behaviour for STI testing relies on overseas data. Due to STIs being an issue strongly influenced by social norms and structures, and New Zealand having a unique ethnic, cultural and social make-up, the assumption that this information is translatable may not be valid. 
Research is urgently required to understand the healthcare-seeking behaviours of New Zealanders with regards to STI testing.

This research is timely because the opportunities for clinician-initiated testing among women are about to become less frequent in New Zealand. The context of a cervical screening consultation lends itself well to broaching the topic of sexual health and it is simple to take a cervical swab at the same time as conducting the cervical smear test. However, the minimum age for smear tests is soon due to increase from 20 years to 25 years of age when the National Cervical Screening Programme changes its protocol to primary human papillomavirus (HPV) testing in 2018 (Primary HPV Screening, 2016). As a consequence, there will be less opportunity for clinicians to offer a screen to those most at risk for STIs (females under 25 years old). Therefore, the need to understand patient-driven motivations and barriers to testing is paramount at this time, and this thesis aims to contribute to that understanding.

\subsection{Research question, aims and objectives}

This thesis is guided by the principal research question: When do people seek healthcare for STI testing in New Zealand and what are the influencing factors involved?

The aims and objectives of this research are to:

i. Provide a background literature review on STIs and healthcare-seeking behaviour, focussing on research relevant to this thesis (Chapter 2)

ii. Quantify the time to presentation for STI testing in people with STI symptoms, identify factors associated with delayed healthcare-seeking, and assess transmission risk in consequence of this behaviour (Chapter 3)

iii. Identify the barriers which prevent or delay people presenting for an STI test (Chapter 4)

iv. Understand the personal reasons for getting an STI test (Chapter 5)

v. Develop a short STI knowledge measure to assess the level of STI knowledge among New Zealanders and relate it to STI testing behaviour (Chapter 6) 
vi. Conduct a systematic review to collate and critically evaluate the published evidence regarding the incidence of reactive arthritis after STI (Chapter 7)

\subsection{Explanation of research method approach}

Mixed methods research describes the process of collecting, analysing and integrating quantitative and qualitative data (Creswell et al., 2004). Mixed methods research is ideal for investigating complex processes and systems in health and healthcare (Fetters et al., 2013). Several prominent researchers have called for studies using a combination of quantitative and qualitative methods to better understand healthcare-seeking behaviour (Ward et al., 1997, Zakher and Kang, 2008, Mapp et al., 2016). A mixed methods approach was chosen as the most appropriate for this research, as some objectives require population data to assess proportions and associations and other objectives need more in-depth data regarding individual experience, beliefs and attitudes. Mixed methods research allows multidimensional insights into symptom experiences and healthcare-seeking whilst offsetting weaknesses of quantitative and qualitative methods (Mapp et al., 2016). This type of research is labour intensive as it involves multiple stages of data collection and analysis (Creswell et al., 2004). However, both types of data are necessary to fulfil the aims and objectives of this study.

There are many approaches to conducting mixed methods research. This work took the form of a three-phase sequential approach, with focus group qualitative work being conducted first (Appendix B), followed by quantitative data collection, followed by qualitative interviews (Figure 1.1). Integration took place at several points: the results of the focus groups directly informed the recruitment protocol for the quantitative data collection; quantitative phase participants formed the sample from which qualitative interview participants were selected; and both quantitative and qualitative data were used to draw overall conclusions in this thesis (Figure 1.1). 


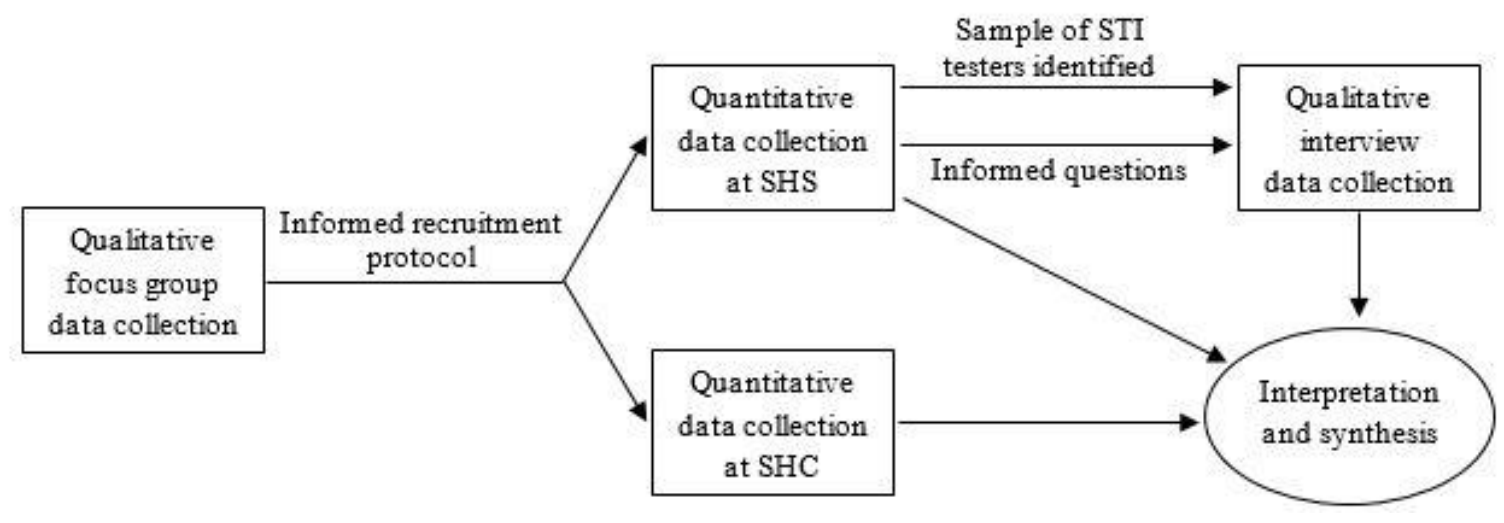

Figure 1.1. Mixed methods framework

\subsection{Thesis structure}

\section{Chapter 1 - Introduction, aims and outline of the thesis}

This chapter gives a brief introduction to the thesis, situating its motivation and aims, and includes a description of the thesis structure.

\section{Chapter 2 - Literature review}

This chapter provides context and background for the thesis by reviewing STIs and their sequelae, as well as STI risk factors. Existing knowledge about healthcare-seeking behaviour from both qualitative and quantitative research disciplines is examined, and an overview of STI testing and treatment paradigms is given. 
Chapter 3 - Healthcare-seeking behaviour of people with STI symptoms attending an urban Sexual Health Clinic (manuscript in preparation for submission. Results from this chapter were presented as an oral presentation at the Sexual and Reproductive Health and Rights Conference Aotearoa New Zealand, Wellington, November 2016)

This chapter describes a study $(n=239)$ which: (i) examines reasons for STI testing among people attending a sexual health clinic; (ii) assesses time to presentation for STI testing in people with STI symptoms; (iii) explores factors associated with delayed healthcare-seeking; and (iv) assesses transmission risk in the period between the onset of symptoms and seeking healthcare.

Chapter 4 - Barriers to sexually transmitted infection testing in New Zealand: a qualitative study (Denison et al. - manuscript under journal review)

This chapter describes the results of qualitative interviews with 24 New Zealand university students about the barriers to STI testing, and these are compared with barriers from studies internationally.

Chapter 5 - "It's like brushing your teeth": University students' reasons for STI testing (Denison et al. - manuscript under journal review. Results from this chapter were presented as an oral presentation at the Sexual and Reproductive Health and Rights Conference Aotearoa New Zealand, Wellington, November 2016)

In this chapter, data from the qualitative interviews $(n=24)$ are used to investigate the reasons people at risk of STIs seek testing. It details important drivers for STI testing and uses illustrative quotes to highlight these.

Chapter 6 - STI knowledge and its association with STI testing behaviour (manuscript in preparation for submission. Results from this chapter were presented as a poster at the World STI \& HIV Congress, Brisbane, September 2015) 
This chapter describes a study $(n=582)$ which: (i) compares the level of STI knowledge between university students and sexual health clinic attendees; (ii) investigates factors associated with STI knowledge; (iii) examines the association between STI knowledge and STI testing history; (iv) compares self-assessed STI knowledge level to measured knowledge level; and (v) investigates the association between campaign awareness and STI knowledge level.

Chapter 7 - The incidence of sexually acquired reactive arthritis: a systematic literature review (This chapter has been published -Denison et al. Clinical Rheumatology, 2016; 35(11): 2639-2648. Results were also delivered as an oral presentation at the New Zealand Rheumatology Association Annual Meeting, Napier, August 2016, and a poster presentation at the American College of Rheumatology Annual Meeting, Washington, November 2016)

This chapter uses systematic literature review methodology to collate and critically evaluate the published evidence regarding the incidence of reactive arthritis after STI, with a view to understand how common it is as a potential pathway to care.

\section{Chapter 8-Discussion and conclusions}

Chapter 8 summarises the main findings of the thesis, discusses the strengths and limitations of the research presented, outlines the public health implications and makes recommendations for future research priorities.

\section{Appendices}

The appendices include additional information relating to the methods and results of the thesis, including the questionnaire, details about development of the recruitment protocol through focus group discussions, supplementary tables for Chapter 6, and the ethical approval documentation. 


\section{Chapter 2 Literature review}

\subsection{Introduction}

This chapter provides background and context for the subsequent chapters of this thesis. After an overview of STIs and their associated sequelae (section 2.2), there follows a description of STI risk factors and incidence rates both internationally and in New Zealand (section 2.3). The chapter also reviews the factors which influence the spread of an STI in a population, with a particular focus on the duration of infection (section 2.4). The review then goes on to highlight the importance of healthcare-seeking behaviour in reducing the duration of infection and thus curtailing the spread of STIs, with literature concerning delay behaviour and the factors influencing healthcare-seeking behaviour also reviewed (section 2.5). Finally, a brief overview of STI testing processes and treatment regimens is presented (section 2.6).

\subsection{Sexually transmitted infections}

This section comprises an introduction to sexual health and to STIs in particular, including descriptions of the most common infections as well as a brief overview of some emerging STIs. STI sequelae are explored including a synopsis of ReA following infection. The asymptomatic nature of many STIs is also discussed as it is highly relevant to healthcareseeking behaviour for STI testing.

\subsubsection{Sexual health}

Sexual health is an important part of human physical and emotional wellbeing. Most people are sexually active for at least some part of their lives making sexual health relevant to most of the world's population (Fryar et al., 2007, Mercer et al., 2013c, Rissel et al., 2014). Sexual health has gained attention from public health practitioners and health service providers because of its contribution towards overall health and wellbeing (World Health Organization, 2015). 
The World Health Organization (WHO) defines sexual health as

“...a state of physical, emotional, mental and social wellbeing in relation to sexuality; it is not merely the absence of disease, dysfunction or infirmity. Sexual health requires a positive and respectful approach to sexuality and sexual relationships, as well as the possibility of having pleasurable and safe sexual experiences, free of coercion, discrimination and violence. For sexual health to be attained and maintained, the sexual rights of all persons must be respected, protected and fulfilled." (World Health Organization, 2002)

From a public health standpoint, this definition shows that the term 'sexual health' encompasses a range of public health and clinical issues. STIs are one such issue which can impact on the four domains of wellbeing mentioned in the WHO definition: physical; emotional; mental; and social, and are the subject of this thesis.

\subsubsection{Types of sexually transmitted infections}

STIs are defined by the WHO as infections that are spread primarily through person-toperson sexual contact. There are more than 30 different sexually transmissible bacteria, viruses, and parasites (World Health Organization, 2013). The most common conditions that STIs cause are chlamydia, gonorrhoea, syphilis, trichomoniasis, genital herpes, genital warts, HIV infection and hepatitis A, B, C and D. Other infections which affect the genital area, such as candidiasis and bacterial vaginosis (BV), can be aggravated by sexual activity but are not considered STIs, so will not form part of this thesis. See Table 2.1 for STI information including the causative agent, type, infection site, symptoms, and incubation time. 
Table 2.1. Common sexually transmitted infections

\begin{tabular}{|c|c|c|c|c|c|}
\hline STI & Causative agent & $\begin{array}{l}\text { Type of } \\
\text { infection }\end{array}$ & Infection site(s) & Symptoms & $\begin{array}{l}\text { Incubation } \\
\text { time }\end{array}$ \\
\hline Chlamydia & Chlamydia trachomatis & Bacterial & $\begin{array}{l}\text { Cervix, fallopian tubes, } \\
\text { pharynx, rectum, male urethra }\end{array}$ & $\begin{array}{l}\text { Discharge, intermenstrual bleeding, vulvovaginitis, cervicitis, } \\
\text { urethritis, epididymitis, epididymo-orchitis, proctitis, prostatitis }\end{array}$ & 7 to 21 days \\
\hline Genital herpes & Herpes simplex virus & Viral & $\begin{array}{l}\text { Labia minora, urethral meatus, } \\
\text { vaginal introitus, penis shaft } \\
\text { and glans, perineum, rectum }\end{array}$ & Lesions, labial oedema, swelling, dysuria, proctitis & 2 to 12 days \\
\hline Genital warts & $\begin{array}{l}\text { Human papillomavirus } \\
\text { (low risk types) }^{\alpha}\end{array}$ & Viral & $\begin{array}{l}\text { Cervix, vulva, vaginal wall, } \\
\text { penis, scrotum, perineum, } \\
\text { rectum, thighs, pubic area }\end{array}$ & Condylomata acuminata (genital warts) & $\begin{array}{l}3 \text { weeks to } 8 \\
\text { months }\end{array}$ \\
\hline Gonorrhoea & Neisseria gonorrhoeae & Bacterial & $\begin{array}{l}\text { Urethra, rectum, pharynx, } \\
\text { conjunctiva, endocervix }\end{array}$ & $\begin{array}{l}\text { Discharge, vulvovaginitis, pharyngitis, swollen glans, inguinal } \\
\text { lymphangitis }\end{array}$ & 2 to 5 days \\
\hline Syphilis & Treponema pallidum & Bacterial & $\begin{array}{l}\text { Initial infection sites: vagina, } \\
\text { urethra, mouth, rectum }\end{array}$ & $\begin{array}{l}\text { Primary stage: chancre. Secondary stage: skin rashes, } \\
\text { mucous membrane lesions, and others. Late stage: neurologic } \\
\text { symptoms, paralysis, gradual blindness, and others }\end{array}$ & 10 to 90 days \\
\hline $\begin{array}{l}\text { Non-specific } \\
\text { urethritis }\end{array}$ & $\begin{array}{l}\text { Inflammation of the urethra } \\
\text { where the cause is } \\
\text { unknown. May include } \\
\text { adenovirus, mycoplasma } \\
\text { genitalium, ureaplasma } \\
\text { species and others }\end{array}$ & $\mathrm{n} / \mathrm{a}$ & Urethra & Discharge, dysuria, urinary frequency and urgency, urethritis & $\mathrm{n} / \mathrm{a}$ \\
\hline Trichomoniasis & Trichomonas vaginalis & Protozoal & $\begin{array}{l}\text { Vulva, vagina, endocervix, } \\
\text { urethra, rectum }\end{array}$ & $\begin{array}{l}\text { Discharge, dysuria, urethritis, vulvovaginitis, epididymitis, } \\
\text { prostatitis }\end{array}$ & 4 to 28 days \\
\hline Hepatitis & $\begin{array}{l}\text { Hepatitis } \mathrm{A}, \mathrm{B}, \mathrm{C} \text { and } \mathrm{D} \\
\text { virus }\end{array}$ & Viral & Liver & $\begin{array}{l}\text { Fatigue, nausea, yellowing of skin and eyes, blood disorders, } \\
\text { fever, cirrhosis, liver cancer }\end{array}$ & $\begin{array}{l}\text { Varies by strain } \\
(2 \text { to } 20 \text { weeks })\end{array}$ \\
\hline HIV and AIDS & $\begin{array}{l}\text { Human Immunodeficiency } \\
\text { Virus }\end{array}$ & Viral & CD4 $\mathrm{T}$ cells in the blood & $\begin{array}{l}\text { Initial flu-like symptoms, followed by long clinical latency } \\
\text { period, progressing to AIDS (if left untreated) which may } \\
\text { include rapid weight loss, chronic fatigue, neurologic disorders } \\
\text { and opportunistic infections }\end{array}$ & $\begin{array}{l}\text { Antibodies: } 2 \\
\text { weeks to } 6 \\
\text { months. AIDS: } 3 \\
\text { to } 11 \text { years }\end{array}$ \\
\hline
\end{tabular}

${ }^{\alpha}$ High risk types of HPV can cause cervical intraepithelial neoplasia, squamous cell carcinoma, and adenocarcinoma and usual incubation time is 1 to $20+$ years This table was compiled using information from Blaxhult et al., 1990, Petrin et al., 1998, Brook, 2002, Fatahzadeh and Schwartz, 2007, Juckett and HartmanAdams, 2010, New Zealand Guideline for the Management of Gonorrhoea, 2014, Syphilis - CDC Fact Sheet, 2014, Kissinger, 2015 , Koren and Decker, 2016. 


\subsubsection{Common STIs}

Chlamydia is caused by Chlamydia trachomatis, an obligate, intracellular, non-motile, gram-negative bacterium (Figure 2.1). The name comes from the Greek word "chlamys", meaning "cloak", as C. trachomatis was originally thought to be a protozoan pathogen that cloaked the nucleus of infected host cells. This cloak has since been discovered to be a cytoplasmic vesicle with many individual organisms inside (Byrne, 2003). There are more than 18 known serological variants (serovars) of $C$. trachomatis; serovars $\mathrm{D}$ to $\mathrm{K}$ are primarily responsible for urogenital infections and L1, L2 and L3 can cause lymphogranuloma venereum (infection of lymphatics and lymph nodes as well as anorectum) (Jaramillo-Rangel et al., 2012, Basu et al., 2015). C. trachomatis is the most prevalent bacterial cause of STIs in the world (World Health Organization, 2013).

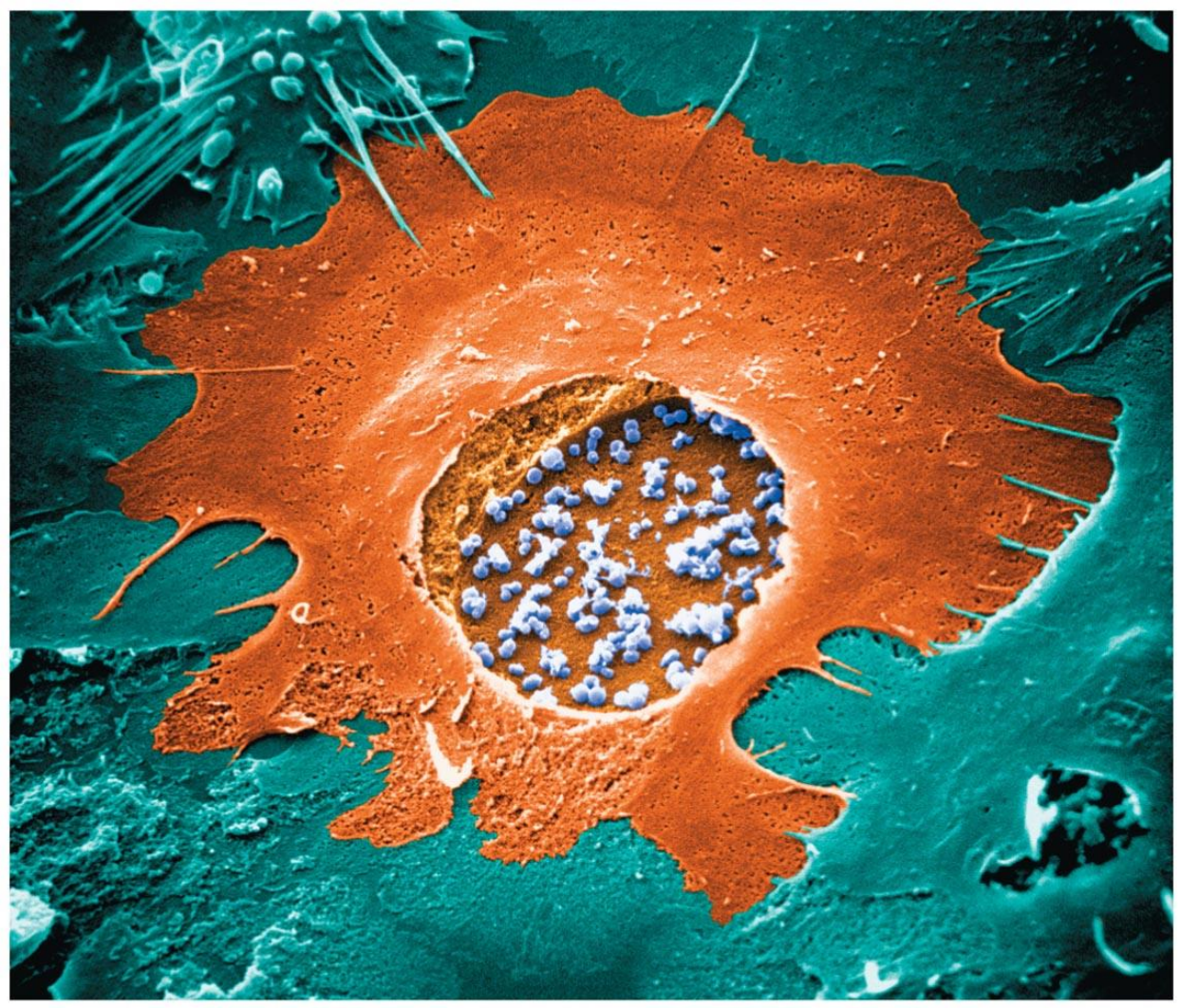

Figure 2.1. Chlamydia (blue) replicating inside vesicle within a cell

Tortora, G J., Funke, B R., Case, C I., Microbiology: an introduction, Global Edition, 1st, O2015. Reprinted by permission of Pearson Education, Inc., New York, New York. 
Gonorrhoea is caused by Neisseria gonorrhoeae, a non-motile, non-spore-forming, intracellular, Gram-negative diplococcus (Figure 2.2). Gonorrhoea has been long-recognised as a human pathogen with biblical references dating back to the Old Testament of the Bible (Oriel, 1994, Morgan and Decker, 2016). It is the second most common bacterial STI after chlamydia (NZSHS, 2014). $N$. gonorrhoeae tends to infect the lower genitourinary tract mucosa, although there is a small risk that the infection may ascend to the upper genital tract in women causing complications such as PID and ectopic pregnancy (Skerlev and CulavKoscak, 2014). In rare cases, haematogenous spread of $N$. gonorrhoeae can lead to disseminated gonococcal infection involving the musculoskeletal system (septic arthritis), cardiovascular system and the skin (NZSHS, 2014). Of serious concern is the increasing $N$. gonorrhoeae resistance to multiple classes of antimicrobials over the past three decades (Cole et al., 2014, Unemo and Shafer, 2014) (see section 2.6.2.1 for more detailed information about antimicrobial resistance).

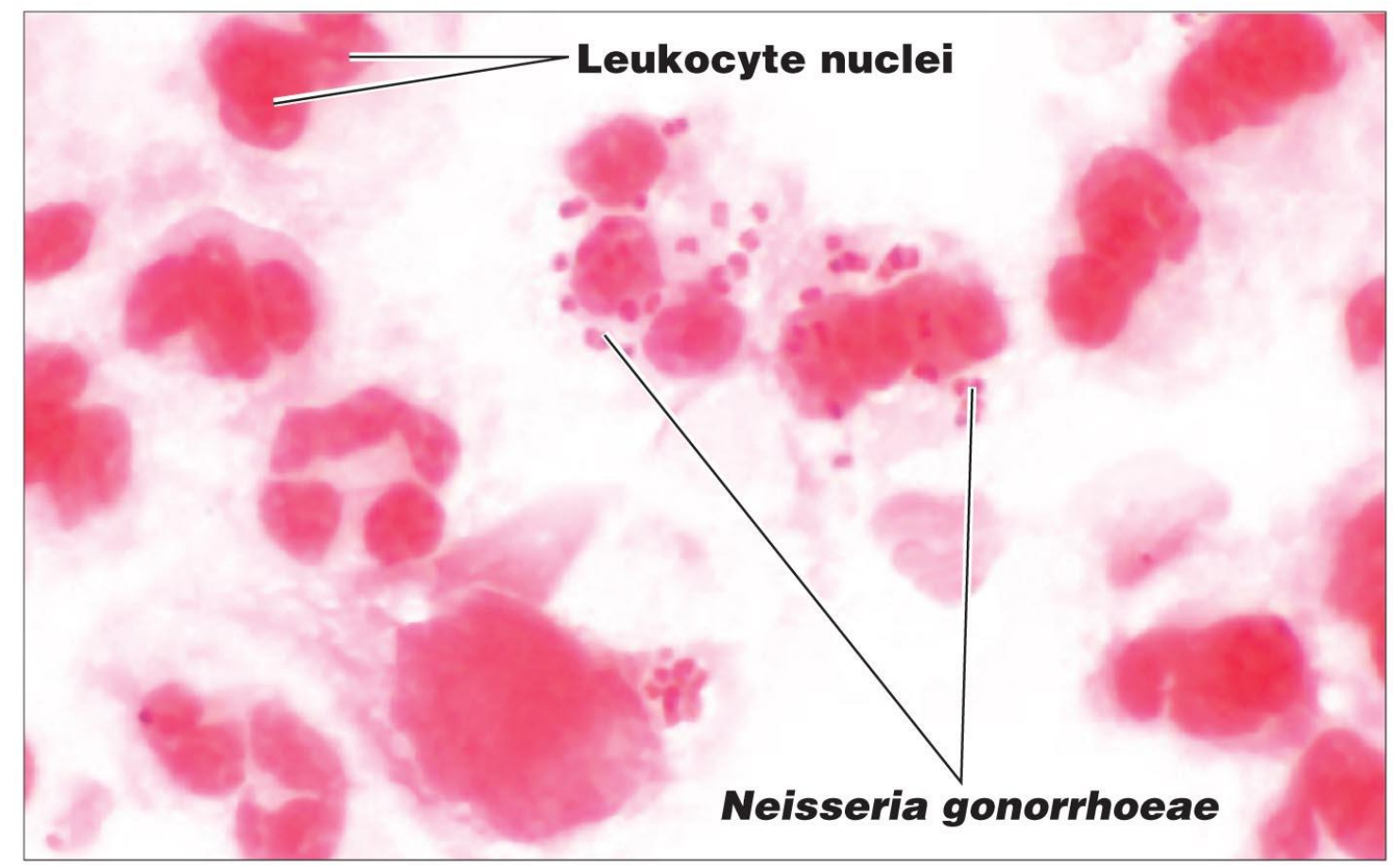

Figure 2.2. Neisseria gonorrhoeae bacteria contained within phagocytic leukocytes Tortora, G J., Funke, B R., Case, C l., Microbiology: an introduction, Global Edition, 1st, (2015. Reprinted by permission of Pearson Education, Inc., New York, New York. 
Human papillomavirus (HPV) is a non-enveloped double stranded DNA virus that causes a variety of conditions including common warts, condylomata (anogenital warts) and multiple malignancies involving the squamous epithelium (Hutter and Decker, 2016). HPV is responsible for the majority of cervical cancers (Cutts et al., 2007), and also a large proportion of penile cancer, vaginal/vulvar cancer, anal cancer, and oropharyngeal cancers. There are over 100 known strains of HPV, around half affect the anogenital areas and at least 13 strains are considered to be oncogenic by the WHO (World Health Organization, 2016b, Hutter and Decker, 2016). The majority of cervical cancers are caused by types 16 and 18 (Durst et al., 1983, Boshart et al., 1984, Bosch et al., 1995). HPV is transmitted through direct skin-to-skin contact, although infection is most often spread through penetrative vaginal or anal intercourse (Braaten and Laufer, 2008).

HPV is the most common STI worldwide, with prevalence figures ranging from $1 \%$ to $73 \%$ quoted in the literature (Tarkowski et al., 2004, Dunne et al., 2006, Dunne et al., 2007, Shikary et al., 2009, Howell-Jones et al., 2012, Steinau et al., 2014). Even the larger figures likely underestimate the cumulative lifetime prevalence of HPV as most infections do not persist (Shikary et al., 2009). Most infections are asymptomatic and self-limiting (Ho et al., 1998). In a study of men with HPV, $2.3 \%$ of genital HPV infections progressed to condylomata (anogenital warts) (Sudenga et al., 2016). Annually, around 500,000 women are diagnosed with cervical cancer worldwide, although rates have decreased markedly over the past couple of decades due to the effectiveness of cervical screening programmes (Vaccarella et al., 2014). However, the incidence of other HPV-associated cancers has increased (Hutter and Decker, 2016).

In 2006, a quadrivalent HPV vaccine was licensed in several countries (Cutts et al., 2007). This is designed to protect against HPV 16 and 18, as well as the low-risk genotypes 6 and 11, which cause $90 \%$ of genital warts. The vaccine has be shown to have more than $90 \%$ protection against persistent infection due to genotypes 16 or 18 in women who received 3 doses of HPV vaccine and have no evidence of past or current infection (Cutts et al., 2007). A 9-valent vaccine has since been developed and was licenced for use in the USA in 2014 (Petrosky et al., 2015). There is no protective effect of these vaccines among women who 
have already been infected with the HPV genotypes they contain. Therefore, it is preferable to vaccinate before sexual debut, making preadolescents the primary target age group.

Genital herpes is a relatively common infection caused by the herpes simplex virus (HSV). HSV are double stranded, enveloped DNA viruses of the Herpesviridae Family (Russell and Crawford, 1963, Garland and Steben, 2014). Genital herpes can be caused by either of two subtypes of the virus, herpes simplex virus type 1 (HSV-1) and herpes simplex virus type 2 (HSV-2). HSV-2 is more commonly associated with genital infection and HSV-1 is more common in orolabial infection (cold sore) (Nahmias and Dowdle, 1968, Koren and Decker, 2016), although both viral subtypes can affect both areas (Langenberg et al., 1999, Garland and Steben, 2014).

HSV is spread via direct contact and infects squamous cells where viral replication takes place. This primary infection may be asymptomatic or result in the vesicular lesions that characterise this infection. Irrespective of clinical symptomatology, after primary infection, HSV can persist within neuronal cell bodies resulting in lifelong latent infection (Fatahzadeh and Schwartz, 2007). HSV may remain dormant or be reactivated to cause recurrent outbreaks of lesions, although the severity and frequency tends to lessen over time (Koren and Decker, 2016). Reactivation may also lead to asymptomatic viral shedding in the absence of clinical disease. Therefore HSV can be transmitted to a sexual partner even when there is an absence of symptoms, although risk of transmission is highest during outbreak periods (Garland and Steben, 2014). As symptoms may be subtle in nature, a significant proportion of patients with genital herpes fail to recognise their symptoms as clinical signs of HSV infection, and so do not seek healthcare (Koren and Decker, 2016).

Trichomoniasis is an infection caused by the protozoan parasite Trichomonas vaginalis. The individual organism is $10-20 \mu \mathrm{m}$ long and 2-14 $\mu \mathrm{m}$ wide and infects the female lower genital tract and the male urethra and prostate (Kissinger, 2015). T. vaginalis infection is associated with reproductive morbidity including low birth weight, preterm delivery, and PID (Silver et al., 2014). In addition, T. vaginalis infection has been shown to be an independent risk factor for HIV acquisition (Hughes et al., 2012, Meites et al., 2015). 
T. vaginalis is more prevalent than C. trachomatis, $N$. gonorrhoeae, and syphilis combined (Kissinger, 2015). However, trichomoniasis is thought to be widely underdiagnosed due to a lack of routine testing, the low sensitivity of a commonly used diagnostic technique (wet mount microscopy), and because the symptoms of trichomoniasis are often sub-clinical or mimic those of other STIs (Petrin et al., 1998, Van der Pol, 2007). Nucleic acid amplification tests (NAATs) and point-of-care tests are now available for T. vaginalis, which will allow for more sensitive testing and screening of both women and men at risk for infection (Meites et al., 2015).

Syphilis is caused by the spirochete bacterium Treponema pallidum and can cause long-term complications if not adequately treated (Centers for Disease Control and Prevention, 2014). Syphilis was first recognized as an STI in the 1400s (Hsiao et al., 2016). Writings from this period suggest that the disease was much more severe than today, with higher and more rapid mortality and was more easily spread, possibly because it was a novel disease and the population had no immunity against it (Frith, 2012).

Syphilis is categorized into different stages of disease based on clinical findings and presentation (Centers for Disease Control and Prevention, 2014). Primary syphilis typically presents with a syphilitic sore, known as a chancre, at the site of infection. This is usually a single sore, but there may be multiple chancres and these typically last a few weeks before healing spontaneously without treatment. Secondary syphilis can present in a variety of ways, and may involve skin rashes (Figure 2.3), mucocutaneous lesions (sores in the mouth, vagina, or anus), swollen lymph nodes, fever, fatigue, weight loss, headaches and hair loss (Eickhoff and Decker, 2016). Without treatment, and after the symptoms of primary and secondary syphilis have resolved, the infection may progress to either latent or tertiary syphilis. Latent syphilis is defined by the absence of clinical manifestations and may last for years to decades. Tertiary syphilis develops in around $15 \%$ of people who have not been treated and can present in a variety of ways (Brown and Frank, 2003, Centers for Disease Control and Prevention, 2014). The disease may damage the internal organs, including the brain, nerves, eyes, heart, blood vessels, liver, bones, and joints. This damage is irreversible and can lead to mental illness, dementia, neurologic deficits including blindness/deafness, heart disease, and even death (Centers for Disease Control and Prevention, 2014). Its wide-ranging effects 
mean that syphilis has been called 'The Great Pretender' as its symptoms can look like many other diseases (Hopkins et al., 2001).

Incidence of and deaths from syphilis dropped rapidly after introduction of penicillin treatment in 1943, however recent years have seen a re-emergence of syphilis mainly affecting men who have sex with men (MSM) and HIV infected individuals (Bremer et al., 2012, Savage et al., 2012, Health Protection Report, 2013). These alarming rises have been partly attributed to changing sexual behaviour (Simms et al., 2005, Savage et al., 2012, Health Protection Report, 2013).

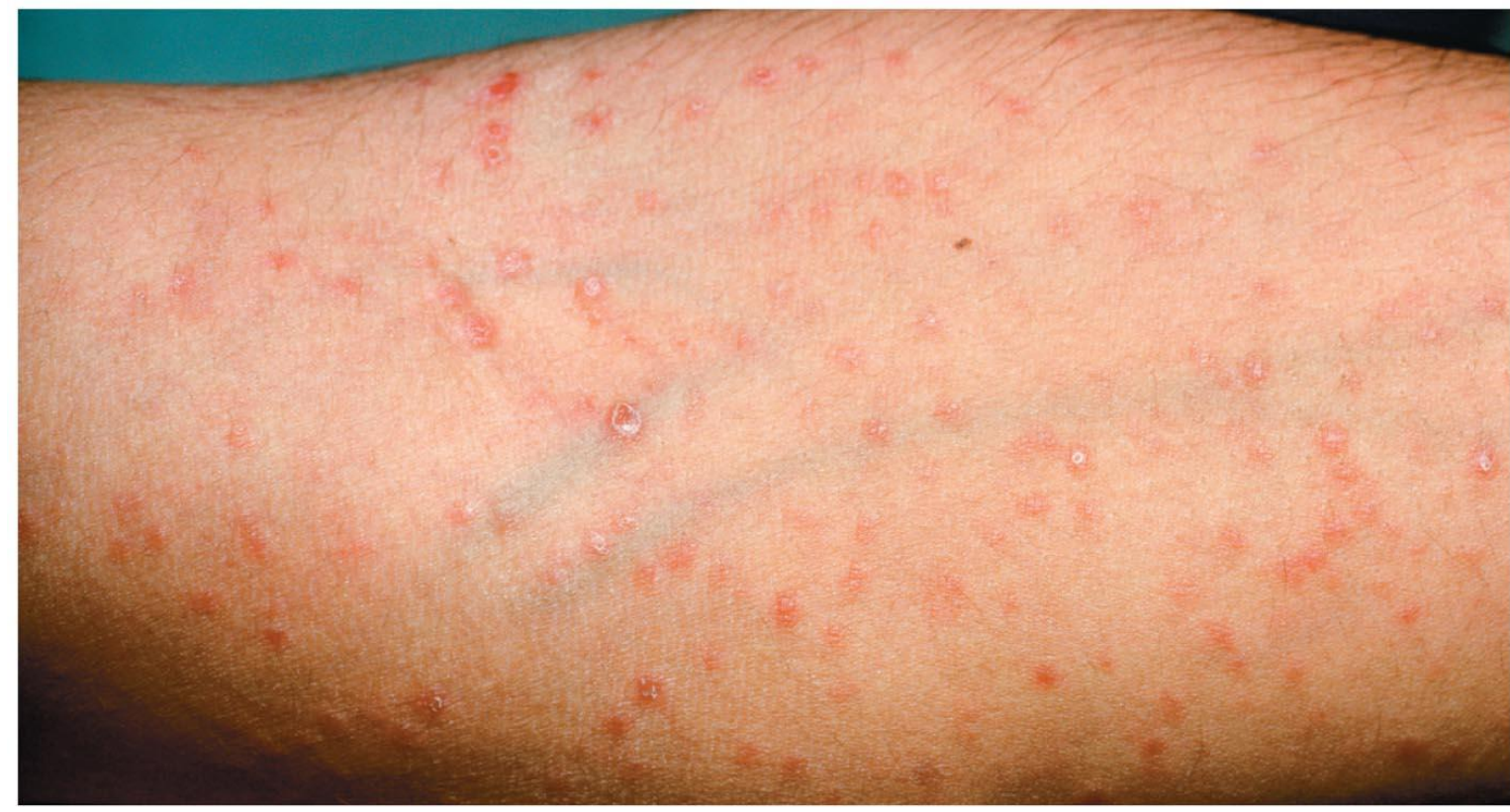

Figure 2.3. Lesions of secondary syphilis rash on a forearm

Tortora, G J., Funke, B R., Case, C l., Microbiology: an introduction, Global Edition, 1st, C2015. Reprinted by permission of Pearson Education, Inc., New York, New York.

Human immunodeficiency virus (HIV) is a retrovirus composed of two copies of positive single-stranded RNA enclosed by a conical capsid of viral protein, housed within a protein matrix and viral envelope (Ganser-Pornillos et al., 2012). HIV infects vital cells in the human immune system such as CD4 T cells, macrophages, and dendritic cells (Figure 2.4), and integrates itself into the cellular DNA (Popovic et al., 1984, Lewis et al., 1992). Once 
integrated, the virus becomes latent, avoiding detection by the body's immune system, or is transcribed, producing new RNA genomes and viral proteins that are packaged and released from the cell as new virus particles, which can then go on to infect more host cells (Manavi, 2006). As HIV has a long clinical latency period (estimated as three to eleven years if left untreated) it may go unrecognised, which is why regular testing of those at risk of acquiring HIV is so important (Blaxhult et al., 1990).

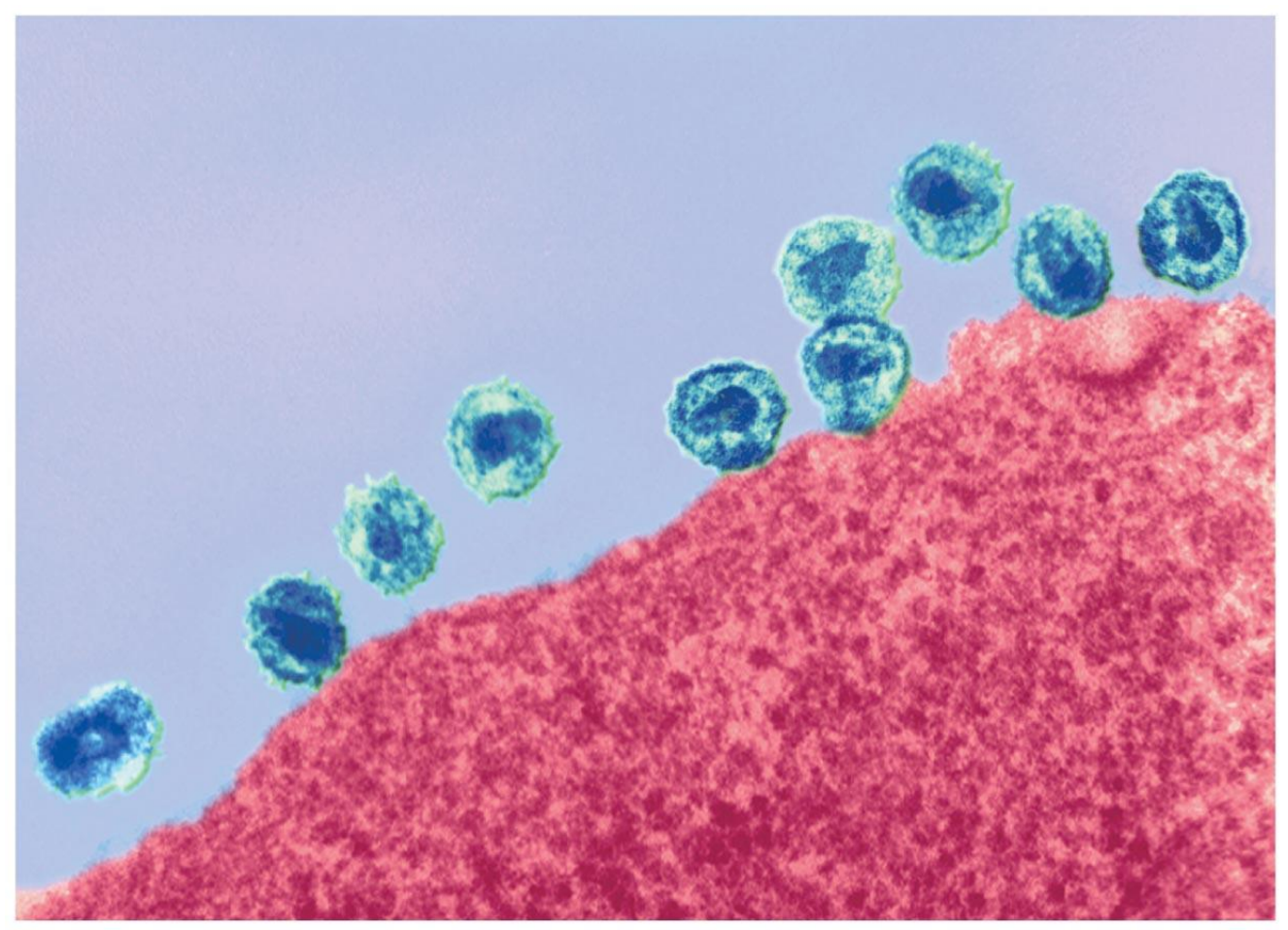

Figure 2.4. Human immunodeficiency viruses infecting a cell

Tortora, G J., Funke, B R., Case, C l., Microbiology: an introduction, Global Edition, 1st, (C2015. Reprinted by permission of Pearson Education, Inc., New York, New York.

HIV destroys CD4 T cells via a number of mechanisms, and if left untreated leads to low levels of these vital immune cells (Manavi, 2006). This makes the body more susceptible to opportunistic infections and malignancies, and when these begin to occur the disease is considered to have progressed to acquired immune deficiency syndrome (AIDS) (Selik et al., 
2014). Opportunistic infections commonly seen in those with AIDS include shingles, candida esophagitis, kaposi's sarcome, non-hodgkins lymphoma and tuberculosis (Selik et al., 2014).

The group most heavily impacted by the sexual transmission of HIV is MSM, in whom the first cases were identified. In every region where data are available, MSM have a higher prevalence of HIV infection than the general population (Beyrer et al., 2012). This is due to several critical factors including the high biological efficiency of HIV transmission through receptive anal intercourse, the sexual partnering dynamics seen in MSM communities, the endemic HIV prevalence in this group, and the sexual role-reversal ability of MSM (Hughes and Saxton, 2015). Other core-groups with a high prevalence of HIV are sex workers, and also intravenous drug users due to the transmission of HIV through needle-sharing (Aral, 2006).

HIV is often diagnosed in the presence of another STI (Ward and Ronn, 2010), this is partly because people who are infected with another STI also tend to be at increased risk of HIV infection due to sexual behaviour, and vice versa (Aral, 2006). Beyond this correlation resulting from common risk behaviours, STIs and HIV may facilitate each other's transmission (Rottingen et al., 2001, Aral, 2006, Ward and Ronn, 2010). There are several plausible biological mechanisms for this. To start with, HIV attacks the host immune system, leaving the individual vulnerable to other infections including STIs (Lazenby, 2012). Conversely, STIs cause inflammation in the genital tract which leads to the recruitment of CD4 T cells (Mlisana et al., 2012), and as these are the host cell of choice for HIV their high concentration facilitates HIV replication in the early stages of infection (Masson et al., 2015). STIs which disrupt the epithelial surface of the genital tract may also increase acquisition of HIV through facilitating access to target cells under the epithelial surface (Ward and Ronn, 2010). In addition, ulcers in both partners, caused by STIs such as HSV, may facilitate blood to blood contact and thereby transmission of HIV (Ward and Ronn, 2010). Effective management of STIs, therefore, may help to reduce the incidence of new HIV infections, however trial evidence has not shown promising results (Ward and Ronn, 2010). 


\subsubsection{Emerging STIs}

An emerging STI is one that has appeared in a population for the first time, or that existed previously but is rapidly increasing in incidence or geographic range, or is an infection that was previously unknown to be sexually transmitted. Recent examples of emerging STIs include Ebola and Zika.

Ebola virus disease (EVD) first appeared in 1976 (Ebola virus infections, 1977, World Health Organization, 1978b, World Health Organization, 1978a) with only small outbreaks occurring until March 2014 when a large outbreak began across multiple countries in West Africa (Fischer and Wohl, 2016). At the time of writing, more than 28,000 people have been infected, with a 40\% mortality rate (Centers of Disease Control and Prevention, 2016). In March 2015, a woman in Liberia received a laboratory-confirmed diagnosis of EVD. Her only potential exposure was unprotected vaginal intercourse with a male survivor of EVD (Christie et al., 2015, Deen et al., 2015). Further investigation found Ebola virus RNA in the survivor's semen 199 days after the onset of his symptoms, with a genetic sequence that matched the sequence from the case patient (where sequencing coverage had been obtained) (Christie et al., 2015). This prompted the WHO and the Centers for Disease Control and Prevention (CDC) to revise their guidelines regarding the length of time that survivors of EVD should avoid unprotected sexual activity (Deen et al., 2015). Subsequently, a pilot study was set-up to investigate the persistence and viability of Ebola virus in the semen of male survivors of EVD (Deen et al., 2015). The results of the study demonstrated that Ebola virus RNA could be detected in the semen of $49 \%$ of the men, and that it could persist to at least nine months after the onset of symptoms. Since the initial case, at least one other occurrence of probable sexually transmission has been reported (Diallo et al., 2016). More research into the sexual transmission of Ebola is required in order to provide better recommendations as to the period of time male EVD survivors should abstain from unprotected sex. There has also been no research into sexual transmission risk in female survivors (Fischer and Wohl, 2016).

Zika virus has been a focus of recent global attention and widespread panic. Zika is a mosquito-borne flavivirus (Barzon et al., 2016), first isolated in 1947 from a rhesus monkey caught in the canopy of Zika Forest in Uganda (Dick et al., 1952). Following this, a small 
number of outbreaks occurred, mainly in the Pacific Islands (Musso and Gubler, 2016). In March 2015, Zika emerged in the Americas and was associated with severe neurological conditions including microcephaly in neonates in Brazil (Musso and Gubler, 2016). Although the main mode of transmission of Zika virus is via mosquito bite, several reports indicate the probable sexual transmission of Zika (Foy et al., 2011, D'Ortenzio et al., 2016, Deckard et al., 2016, McCarthy, 2016, Musso and Gubler, 2016). Infectious Zika virus has been recovered in semen up to 24 days after symptom onset (D'Ortenzio et al., 2016), and viral RNA has been detected for over six months after onset (Barzon et al., 2016, Nicastri et al., 2016). There is also evidence that the viral load may be higher in semen than in blood or urine (Mansuy et al., 2016). The evidence of sexual transmission suggests a mode of interhuman transmission that could be contributing to the spread of Zika observed in the Americas (Musso and Gubler, 2016), although it is unlikely to initiate or sustain an outbreak alone (Gao et al., 2016). Further research into the sexual transmission of Zika is needed in order to direct control efforts to contain the spread of the infection.

\subsubsection{Symptomatic and asymptomatic infection}

Symptoms of an STI may include discharge from the penis or vagina, genital itching or irritation, genital lesions, painful urination (dysuria), pelvic pain and pain during sexual intercourse (see Table 2.1). However, many STIs either do not produce acute symptoms or clinical signs of disease, or do not produce symptoms sufficiently severe for an infected individual to notice (Detels et al., 2011, Dielissen et al., 2013). Asymptomatic infection is more common in women than in men (Malhotra et al., 2013). For chlamydia, often termed 'the silent infection', the literature tends to quote values of approximately $25 \%$ to $50 \%$ of male cases and $70 \%$ to $80 \%$ of female cases being asymptomatic (Malhotra et al., 2013, Davies et al., 2014a). However, these rates may be an underestimate; the National Longitudinal Study of Adolescent Health Study collected data prospectively from 14,322 U.S adolescents and followed them into adulthood. Of the participants who tested positive for chlamydial infection, $95 \%$ did not report symptoms in the 24 hours preceding specimen collection (Miller et al., 2004).

Given the rates of asymptomatic infection, it is not surprising that many STIs go unrecognised. This has serious consequences for infected individuals, as an organism may 
still be causing damage internally in the absence of noticeable symptoms, which can lead to PID and other complications. On a population level, asymptomatic infection has significant implications because asymptomatic infection can be transmitted unknowingly. The proportion of asymptomatic versus symptomatic infection suggests there is a potentially huge population of untreated individuals unknowingly transmitting STI organisms to new partners.

\subsubsection{Sequelae}

STIs can have serious health outcomes, some of which have been mentioned in section 2.2.3. Some sequelae are specific to particular STIs but others can potentially be caused by a range of infections; these will be discussed in more detail in the following sections.

\subsubsection{Pelvic inflammatory disease}

The term pelvic inflammatory disease (PID) encompasses a range of upper genital tract inflammatory disorders in women which result from the intracannicular ascending of microorganisms from the lower reproductive tract (vagina, cervix and vulva) into the upper genital tract (fallopian tubes, ovary and uterus). Several different types of bacteria can cause PID, including C. trachomatis, $N$. gonorrhoeae and genital mycoplasma species. Short-term sequelae include the development of tubo-ovarian abscesses, inflammation of the liver and the appendix. Long-term sequelae include ectopic pregnancy, chronic pelvic pain, and tubal infertility due to scarring (Westrom et al., 1992). PID is also associated with Fitz-HughCurtis syndrome (FHC), which causes fibrous adhesions between the liver and the diaphragm (Stajano, 1920, Curtis, 1930, Fitz-Hugh, 1934), although FHC can also present in men indicating it can occur in the absence of PID (Lopez-Zeno et al., 1985, Nardini et al., 2015). The length of time that PID is left untreated appears to modify the risk of developing complications. A study by Hillis and colleagues (1993) found that women who delayed seeking care for PID were three times more likely to experience infertility or ectopic pregnancy than women who sought care promptly.

It is difficult to accurately determine the proportion of people infected with an STI that go on to develop PID because of the ethical implications inherent in conducting a prospective study where treatment is withheld. A few studies have looked at the short-term natural history between testing and treatment and found that PID occurrence in this interval (median 2 weeks) ranged from $2 \%$ to $4.5 \%$ (Parks et al., 1997, Geisler et al., 2008). Other studies, such 
as the POPI (prevention of pelvic infection) trial, have randomised large numbers of women to screening and treatment or to no screening (Oakeshott et al., 2010). Price et al. (2013) synthesised data from both types of studies in their review and found that the probability that an episode of chlamydia would cause clinical PID was 0.16 (95\% CI, 0.06-0.25).

Retrospective studies using routinely collected data have also been conducted. In a study of 43,715 women in Uppsala, Sweden, the cumulative incidence of PID by age 35 years diagnosed in hospital was $5.6 \%$ in women who had ever tested positive for chlamydia and $4.0 \%$ in those with negative test results (Low et al., 2006). The resulting hazard ratio was 1.27 (95\% CI, 1.04-1.55) for those testing positive compared with those who tested negative after adjustment for potential confounders (Low et al., 2006). A similar study was conducted more recently among 74,000 women in Manitoba, Canada, where diagnoses made in primary care were included in addition to those made in hospital settings (Davies et al., 2014b). This study found an adjusted hazard ratio of 1.55 (95\% CI, 1.43-1.70) in women who tested positive compared with those who tested negative (Davies et al., 2014b). The larger hazard ratio is probably due to the inclusion of less severe cases of PID that had not warranted hospitalisation. Not all women will be at equal risk of PID following infection. Studies have generally found that younger age increases the risk of PID, as does having more sexual partners and experiencing repeated infections (Davies et al., 2014b, Davies et al., 2016, Hay et al., 2016).

\subsubsection{Infertility in men}

STIs play an etiologic role in male infertility also. Bacterial pathogens including $C$. trachomatis, Ureaplasma urealyticum and mycoplasma hominis have been associated with reduced sperm quality (Mazzoli et al., 2010), decreased concentration and motility (Mazzoli et al., 2010, Sellami et al., 2014) and increased apoptosis (Eley et al., 2005a). Some viral STIs have also been implicated, including HPV, hepatitis B, hepatitis C, and HIV (Gimenes et al., 2014). However, studies to date show conflicting results, and whether or not the effects on semen have a direct detrimental effect on fertility is still unclear (Keck et al., 1998, Eley et al., 2005b, Abusarah et al., 2013, Mackern-Oberti et al., 2013, Sellami et al., 2014) It has also been suggested that subclinical infections may activate an immune response that either 
destroys or hinders sperm (Gonzales et al., 2004, Eley et al., 2005b, Jaramillo-Rangel et al., 2012).

\subsubsection{Adverse pregnancy outcomes related to STIS}

Miscarriage is one of the most common adverse pregnancy outcomes worldwide. It is defined as the spontaneous loss of a pregnancy during the first 24 weeks of gestation (though there is some variation in the literature as to the gestational cut-off for miscarriage versus stillbirth). Infection is just one of a number of factors that may cause or contribute to a miscarriage. A review investigating the association of high-risk infections with miscarriage was recently conducted (Giakoumelou et al., 2016). The authors concluded that syphilis and HIV were associated with miscarriage, but there was conflicting evidence for C. trachomatis, HPV, HSV-1 and HSV-2, and HPV. Even for infections where a causative role is established, the underlying molecular cause of miscarriage is still unknown.

Stillbirth is the term used here for spontaneous loss of a pregnancy after 24 weeks of gestation. Maternal infections are thought to be important causes of stillbirth, accounting for half of stillbirths in low- and middle-income and 10-25\% in high-income countries (Goldenberg et al., 2010). Syphilis has been shown to play a role in stillbirths and some studies have also implicated chlamydial infection (Gencay et al., 2000, Mardh, 2002, Goldenberg et al., 2010, Liu et al., 2013, Adachi et al., 2016).

Preterm delivery has been identified as the single most important cause of perinatal morbidity and mortality, accounting for $27 \%$ of the nearly four million reported annual neonatal deaths (Adachi et al., 2016). Preterm delivery is a multi-factorial process which leads to the premature rupture of membranes (World Health Organization, 2012a). Genital tract infections may be important contributing factors, with some suggesting they may contribute to as many as $40 \%$ of preterm birth cases (Pararas et al., 2006). Reviews have found that $C$. trachomatis infection during pregnancy is associated with an increased risk of preterm delivery (Silva et al., 2011, Adachi et al., 2016), and there is also evidence that HIV and $N$. gonorrhoeae may be associated (Johnson et al., 2011, Waight et al., 2013, Xiao et al., 2015). 
Low birth weight at full-term delivery suggests there has been some intrauterine growth restriction, and is associated with long-term health consequences (Barker et al., 1989, Barker, 1995, Whincup et al., 2008). Several STIs have been implicated as a cause of low birth weight, for example, the review article by Silva et al. (2011) found that chlamydial infection during pregnancy was associated with an increased risk of low birth weight (RR 1.52, 95\% CI 1.24-1.87). In Australia, a prospective study of pregnant women in an urban Indigenous community found that low birth weight was associated with the presence of any STI during the pregnancy; this appeared to be largely due to the presence of infectious syphilis (Panaretto et al., 2006).

In addition to these adverse pregnancy outcomes, STI organisms can be passed from mother to child during vaginal delivery causing eye infections, blindness, pneumonia, and in the case of HSV, herpes neonatorum (Rogstad and Omer, 2014, James and Kimberlin, 2015).

\subsubsection{Reactive arthritis}

Reactive arthritis $(\operatorname{ReA})$ is an inflammatory arthritis of the joints or periarticular tissues brought about by an infection at a distant site in the body (Hamdulay et al., 2006). ReA belongs to the group of spondyloarthritides, which also includes ankylosing spondylitis (AS), psoriatic arthritis, arthritis in inflammatory bowel disease and undifferentiated spondyloarthritis (Dougados and Baeten, 2011, Rudwaleit et al., 2011). It can cause pain, stiffness and swelling, and occasionally extra-articular symptoms such as conjunctivitis and oral lesions, including mucosal ulcers. ReA typically occurs in young adults and can affect both men and women (Yu, 2013) though is reported to occur more frequently in men (Carlin et al., 2014). There seems to be a genetic predisposition to ReA, with the HLA-B27 gene being more common in patients with spondylarthritis including ReA (Yu, 2013). In some, but not all studies, the HLA-B27 gene is also associated with increased severity and chronicity of ReA (Pope et al., 2007). HLA-B27 is more common among white individuals, which in part explains the higher incidence of ReA in this group (Reveille et al., 2012).

ReA usually develops one to four weeks after an acute infection, and often causes only mild symptoms which remit spontaneously (Hamdulay et al., 2006). However, up to 18\% of those affected develop a chronic arthritis where symptoms persist for longer than six months (Hannu et al., 2006). Although not life-threatening, this condition has a significant disease 
burden (Gibney et al., 2014) especially as ReA primarily affects a working age population, meaning even a transient disruption of an individual's life is a personal and economic problem.

It is reported that ReA can be triggered by a wide spectrum of microbial agents including bacteria as well as viruses and parasites (Ozgul et al., 2006, Espinoza and Garcia-Valladares, 2013). Data from retrospective studies of ReA patients suggest that the most frequent microbial triggers are those that infect the gastrointestinal or genitourinary tracts (Hamdulay et al., 2006). ReA resulting from an STI has been termed Sexually Acquired Reactive Arthritis, or 'SARA'. C. trachomatis, the most common bacterial STI in New Zealand, is proposed to be the most common cause of ReA (Keat et al., 1987, Kvien et al., 1994, Morris and Inman, 2012, Singh and Karrar, 2014). Other STIs including N. gonorrhoeae (distinct from its role in septic gonococcal arthritis) and Ureaplasma urealyticum are also implicated (Carlin et al., 2014). ReA is commonly reported in the setting of HIV infection, yet it is not known whether HIV itself causes ReA or whether the elevated rates reported in HIV-positive individuals are because their immunocompromised status makes them more vulnerable to opportunistic infections which may then trigger ReA. In addition, the risky sexual behaviours associated with HIV acquisition put this population at higher risk of acquiring other STIs which may trigger ReA. A recent review of the association between HIV and ReA found that the epidemiological data were too complex and variable to draw clear conclusions about the relationship between HIV infection and ReA (Lawson and Walker-Bone, 2012). Despite the high prevalence of STIs such as chlamydia, the incidence rate of ReA after STI has not been well studied.

\subsubsection{Psychological impact of diagnosis}

In addition to physical sequelae, being diagnosed with an STI can cause significant psychological distress (Melville et al., 2003, Mills et al., 2006, Piercy, 2006a). The social construction that having an STI indicates a person has engaged in immoral behaviour can cause feelings of shame and self-stigma. People who have been diagnosed with an STI often report a strong sense of bodily contamination, stating that they feel 'dirty' (Gotz et al., 2005, Piercy, 2006a). This feeling may not go away until retesting has occurred and the individual has received a negative test result (Piercy, 2006b). 


\subsection{Burden of disease and factors that increase STI risk}

To place this thesis in context, the incidence and prevalence of STIs globally, and more specifically in New Zealand, are explored. Trends over recent years are also described and potential explanations for these suggested. In addition, this section considers the various risk factors for STI, including demographic characteristics, risk behaviours and societal determinants.

\subsubsection{Rates of STIs}

The true incidence and prevalence of STIs are not known because many infections are asymptomatic and remain undiagnosed. Large-scale cross-sectional population studies are one way to estimate prevalence, however there are many challenges to achieving a truly representative sample, most notably responder bias. In addition, the majority of chlamydia and gonorrhoea cases included in cross-sectional studies and surveillance statistics are vaginal and urethral, because these are the usual sites swabbed in asymptomatic testing. However, it is likely that extra-genital infections are being missed. One study recently reported that the rate of rectal gonorrhoea among women who report anal intercourse may be similar to that of urogenital infections (Javanbakht et al., 2012).

The WHO has regularly produced estimates of the global and regional prevalence of four curable STIs (chlamydia, gonorrhoea, trichomoniasis and syphilis), based upon literature reviews of prevalence data, since 1995 (Gerbase et al., 1998, World Health Organization, 2001, World Health Organization, 2011b, World Health Organization, 2012c, Newman et al., 2015). The 2012 global estimates of STI prevalence and incidence are the latest to be published and are based on data collected between 2005 and 2012 (Newman et al., 2015). The estimated pooled prevalence for chlamydia, gonorrhoea, trichomoniasis and syphilis are presented in Table 2.2. These figures are only estimates however, as many assumptions and adjustments had to be made where data was lacking and there was high heterogeneity in methodology between studies. In addition, STI co-infection was not taken into account and the analysis was restricted to urogenital infections only. 
Table 2.2. Estimated global prevalence of four STIs (95\% uncertainty interval shown in parentheses)

\begin{tabular}{lcccc}
\hline & Chlamydia & Gonorrhoea & Trichomoniasis & Syphilis \\
\hline Female & $4.2 \%(3.7-4.7 \%)$ & $0.8 \%(0.6-1.0 \%)$ & $5 \%(4.0-6.4 \%)$ & $0.5 \%(0.4-0.6 \%)$ \\
Male & $2.7 \%(2.0-3.6 \%)$ & $0.6 \%(0.4-0.9 \%)$ & $0.6 \%(0.4-0.8 \%)$ & $0.5 \%(0.3-0.7 \%)$ \\
\hline
\end{tabular}

New Zealand lacks general population prevalence studies (Morgan, 2013). However, data are available for chlamydia infection from convenience samples and sub-populations such as the military, clinic attendees and pregnant women (Cole et al., 2001, Lawton et al., 2004, Sparrow et al., 2007). A study of under 25 year olds attending Family Planning Clinics (FPCs) in Wellington found a prevalence of $8 \%$ when they offered chlamydia urine testing to all attendees across a six month period in 2004 to $2005(\mathrm{n}=2533)$ (Sparrow et al., 2007). A positive result was more likely in those with a history of partner change, in those who didn't always use condoms, or in Māori and Pacific peoples. In a study conducted at Victoria University of Wellington in 2003, the prevalence of undiagnosed chlamydia among women attending the Student Health Services for any reason was found to be 2.7\%, and was associated with previous STI, non-European ethnicity, and irregular use of condoms ( $\mathrm{n}=$ 715) (Baker et al., 2005). The heterogeneity of these studies and of the populations of interest limits their comparison to each other, and also to the estimated global rates as calculated in the 2012 WHO publication.

The WHO publication (Newman et al., 2015) also estimated incidence rates from the prevalence rates identified in their study. The estimated global incidence rate for chlamydia was 38 per 1,000 in women and 33 per 1,000 in men, for gonorrhoea the rate was 19 per 1,000 in women, and 24 per 1,000 in men, for trichomoniasis 38 per 1,000 in women and 40 per 1,000 in men, and for syphilis 1.5 per 1,000 in women and 1.5 per 1,000 in men. These incidence rates translated into 357 million new infections in adults that year, or an average of nearly one million new infections per day. The 2012 estimate of the number of new infections was lower than the 2005 and 2008 estimates (448 and 498, respectively). However 
the epidemiological and statistical methods used have changed over time so differences in the estimates between reports cannot be interpreted as trends over time.

Surveillance data can give some indication of country-specific trends. Direct comparison between countries is difficult because surveillance methods differ by country depending on reporting policies, healthcare infrastructure, and available resources. Assessing trends over time even within the same country is also challenging due to developments in surveillance techniques causing data between different years to be non-equivalent. Short-term time-trend analysis is more robust than long-term, as there will be fewer changes in methodology over shorter time periods. Recent data suggest that some infections may be on the increase in developed countries. The CDC in the USA reported that the incidence of chlamydia, gonorrhoea and syphilis increased by $2.8 \%, 5.1 \%$ and $15.1 \%$ respectively in 2014 compared to 2013 (Centers for Disease Control and Prevention, 2015). Similar rises in the rates of gonorrhoea and syphilis have been seen in the UK, although the incidence of chlamydia has been reducing since 2013 (Health Protection Report Volume 10 Number 22, 2016). In Australia, chlamydia cases increased steadily between 2006 and 2011 and have since remained stable, however gonorrhoea cases continue to rise. The rate of diagnosis of infectious syphilis cases has tripled for men, but remains low for women (The Kirby Institute. Annual Surveillance Report, 2016). The recent increase in rates of gonorrhoea and syphilis seem to be largely due to increases in incidence among MSM. It has been suggested that this change is because of more frequent risky sexual behaviour among MSM, potentially due in part to improved therapies for HIV (Beltzer et al., 2013).

To date, STIs have not been mandatorily notifiable in New Zealand, although this is about to change for some STIs. Routine surveillance in New Zealand has been based on voluntary reporting from clinics and participating laboratories and data are analysed and reported by The Institute of Environmental Science and Research (ESR). Only recently, in 2013, did all the regional health service provider organisations (the District Health Boards, or DHBs) and laboratories in New Zealand provide STI surveillance data for a full year. Over the past few years, changes have been made to enhance and improve the surveillance of STIs in New Zealand. These improvements have allowed the reporting of population-based rates of chlamydia and gonorrhoea, for analyses comparing different population groups, and for the 
removal of duplicates where one person has multiple positive specimens per accession. However, these changes mean that direct comparison of incidence rates across time is not valid. Recently, a Health Protection Amendment Bill has been passed by parliament which makes HIV, gonorrhoea, and syphilis notifiable on a non-identified basis, along with AIDS. Chlamydia is noticeably absent. The Bill came into effect in January 2017 and will substantially strengthen the New Zealand surveillance system, providing more robust and accurate data for the included infections.

Based on national laboratory data, the estimated national rate of chlamydia (based on all DHBs where data was available) was stable between 2009 and 2011 but has decreased since 2012 (Figure 2.5) (ESR Annual Surveillance Report 2014). Similarly to other countries, the estimated national gonorrhoea rate increased from 2010 to 2012, though decreased in 2013 and again in 2014 (Figure 2.6). Some of the decrease seen in 2013 is likely to be attributable to the new data processing methods introduced by ESR that year. The 2014 national rate of chlamydia was 869 per 100,000 population for women and 375 per 100,000 population for men (ESR Annual Surveillance Report 2014). When restricting the analysis to those between 15 and 24 years old, the rate was 2990 per 100,000. For gonorrhoea, the 2014 national rate was 62 per 100,000 population for women and 77 per 100,000 population for men, and when restricting to 15 to 25 year olds, the rate was 278 per 100,000 population. Although it is important to remember that these figures are probably an underestimate as they are based on the number of diagnosed cases, which are only the tip of the iceberg, as many infections go undiagnosed. 


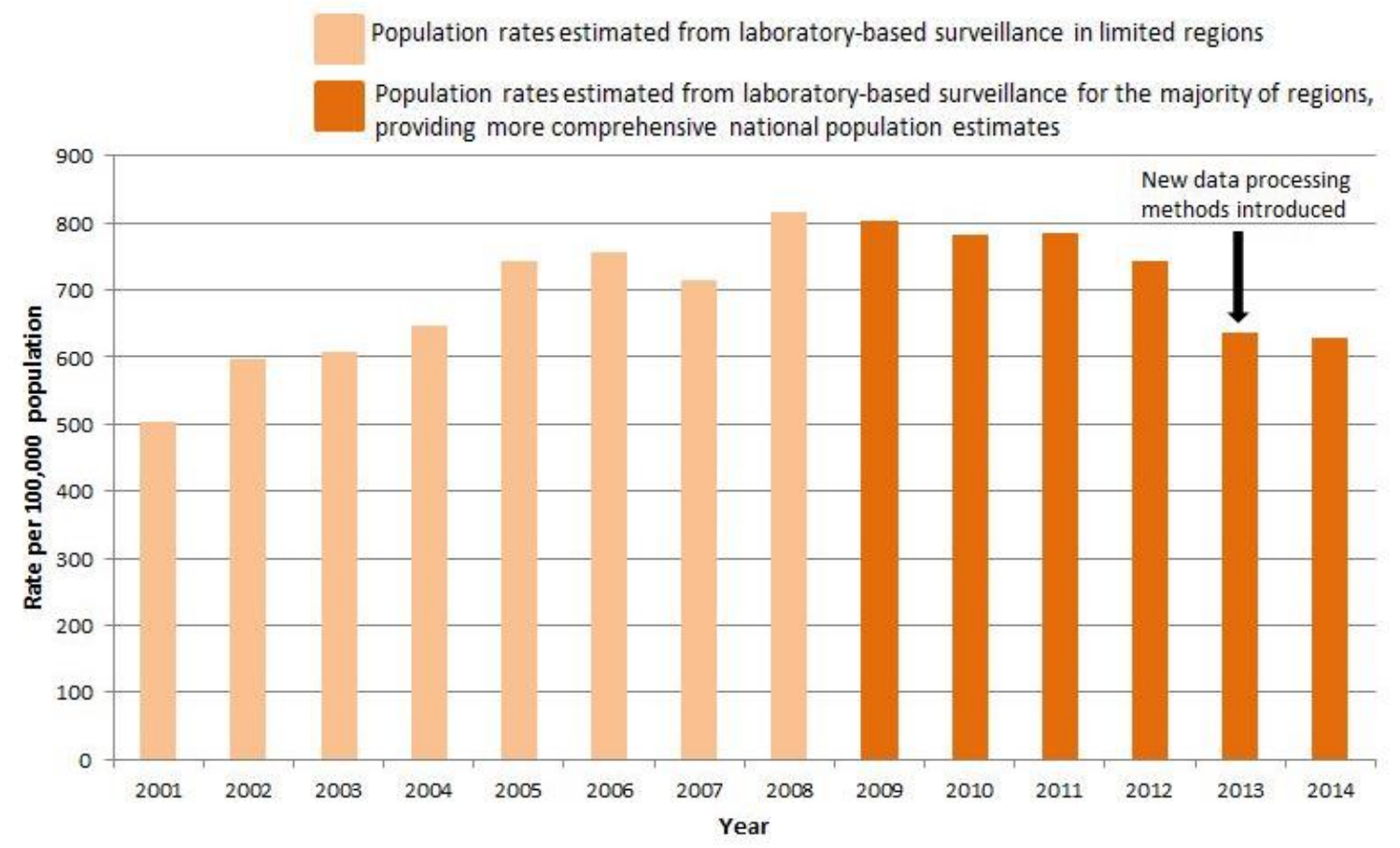

Figure 2.5. National chlamydia rate per 100,000 population over time Produced using data from The Institute of Environmental Science and Research

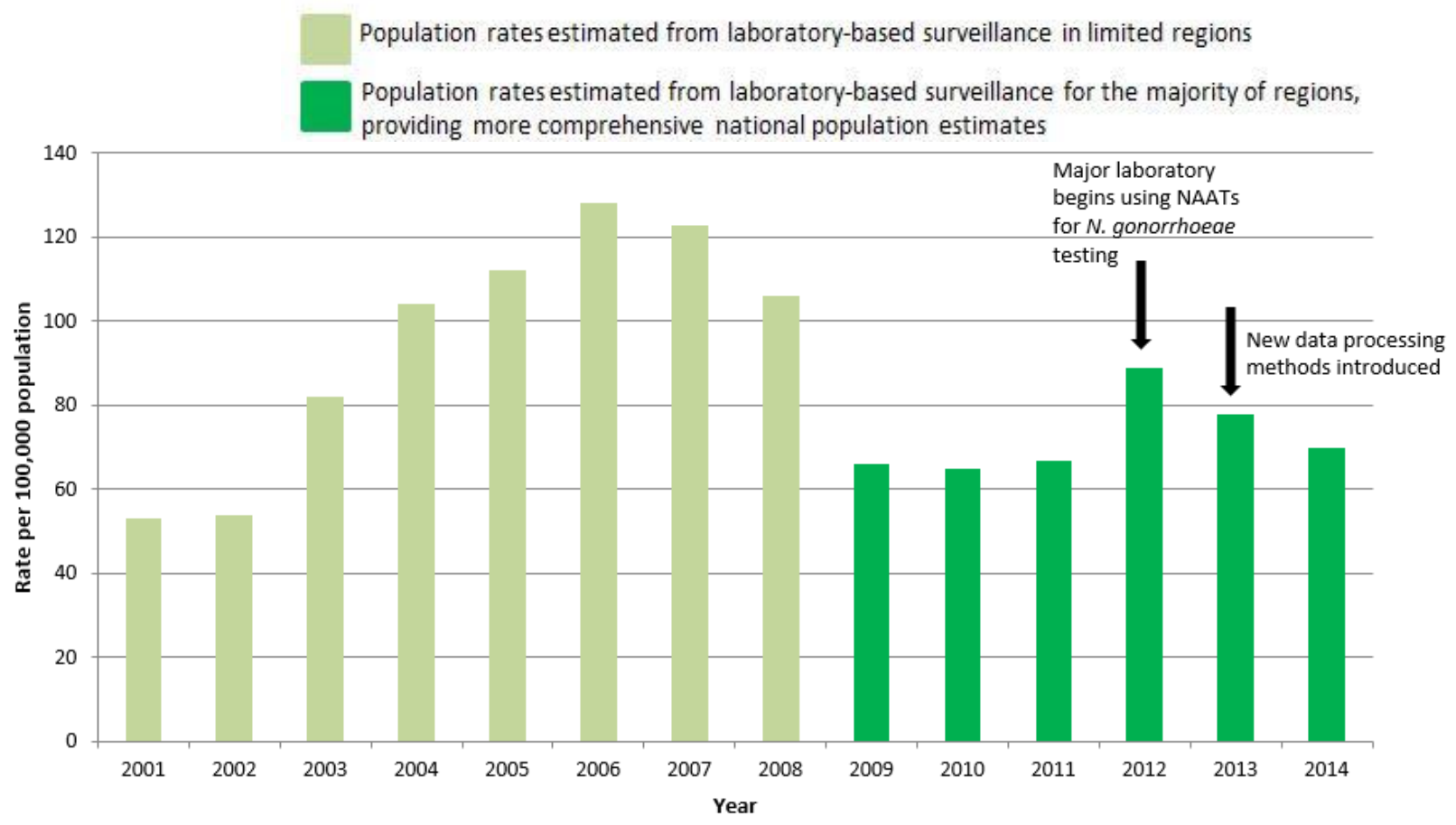

Figure 2.6. National gonorrhoea rate per 100,000 population over time Produced using data from The Institute of Environmental Science and Research 
Increases in incidence and prevalence figures for chlamydia and gonorrhoea may be partly due to expanded use of more sensitive diagnostic tests for the detection of their etiologic organisms. Laboratory diagnosis was traditionally performed by cell culture or antigen detection for C. trachomatis, and culture for N. gonorrhoeae. More recently, NAATs have become widely used; these tests have a greater sensitivity in both symptomatic and asymptomatic infection than traditional methods and so have improved the ability to detect C. trachomatis and N. gonorrhoeae infections (Van Dyck et al., 2001, BASHH, 2012). Therefore it is likely that they will have some effect on reported incidence rates. In New Zealand, a study examined the influences on testing and detection trends of C. trachomatis and N. gonorrhoeae in Waikato, a region in the upper North Island, during 1998 to 2006 (Morgan, 2008). At the time, NAAT was being used at two of three laboratories in the region, with the third continuing to process a substantial number of $C$. trachomatis tests using non-NAAT techniques until the end of 2004. The data did not show a clear impact on the positivity rate from the introduction of more sensitive chlamydia NAAT testing at the third laboratory. The Ministry of Health in New Zealand also reported that between 1995 and 2000 when NAATs were being introduced for $C$. trachomatis testing, that the number of chlamydia cases had also risen dramatically at sexual health clinics (SHCs) not using NAATs, suggesting they are not entirely responsible for the rise (Ministry of Health. Sexual and Reproductive Health, 2003). NAATs were introduced for $N$. gonorrhoeae testing in New Zealand between 2009 and 2013. Increases in regional gonorrhoea rates are evident in the surveillance data after the main or sole testing laboratory in that region changed to using NAAT. This suggests that the introduction of NAATS has had an effect on the number of cases detected and is at least partially responsible for the increase in incidence rates seen over the past few years in New Zealand.

Syphilis rates in New Zealand are monitored by clinic-based reporting. From 2010 to 2014, the number of syphilis cases reported by SHCs increased by $16.7 \%$ (from 120 to 140 cases) (ESR Annual Surveillance Report 2014). Enhanced surveillance of infectious syphilis, which collects information on sexual behaviour and other possible risk factors, suggests that the increase in cases is driven by transmission amongst MSM (ESR Annual Surveillance Report 2014) which reflects the situation being reported internationally (Bremer et al., 2012, The 
Kirby Institute. Annual Surveillance Report, 2016, Health Protection Report Volume 10 Number 22, 2016).

National surveillance of HIV and AIDS is conducted by the AIDS Epidemiology Group at the Dunedin School of Medicine, University of Otago. In 2015, 224 people (205 men, 18 women, and one transgender woman) were diagnosed with HIV in New Zealand (AIDS Epidemiology Group, 2016). Of these, 39 had previously been diagnosed overseas. This is the highest number of new cases diagnosed in New Zealand in a single year, though the infection may have occurred a number of years prior to diagnosis so this figure does not represent an infection incidence rate. This rise is driven by increasing numbers of HIV diagnoses in MSM. Overall, the number of MSM infected with HIV in New Zealand has been rising for the past four years, whereas there has been a downward trend in HIV diagnoses among heterosexual men and women (AIDS Epidemiology Group, 2016). The HIV/AIDS epidemic in New Zealand meets the UNAIDS/WHO criteria for a 'low-level' epidemic, as infection is largely confined to individuals with higher risk behaviour, and HIV prevalence has not consistently exceeded five percent in any defined sub-population (AIDS Epidemiology Group, 2012).

Country comparison is problematic due to the differing methodologies used in data collection and analysis. However, a crude comparison of the published figures from 2014 (ESR Annual Surveillance Report 2014, The Kirby Institute. Annual Surveillance Report, 2015, Centers for Disease Control and Prevention, 2015, Public Health England, 2016) indicates that the rate of chlamydia in New Zealand is 1.4 to 1.7 times higher than rates reported from Australia, the USA and England (Figure 2.7) but the rate of gonorrhoea is similar to rates observed in Australia and the UK and around two-thirds of that in the USA (Figure 2.8). 


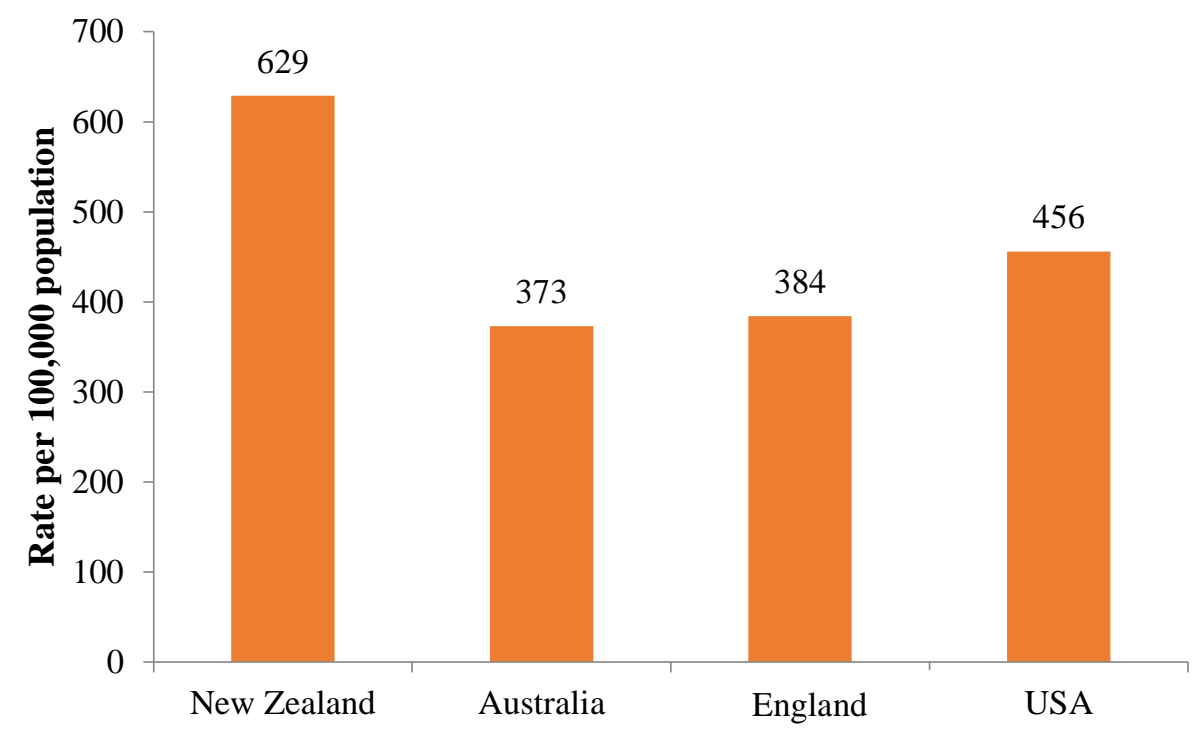

Figure 2.7. Rates of chlamydia per 100,000 population for four developed countries in 2014

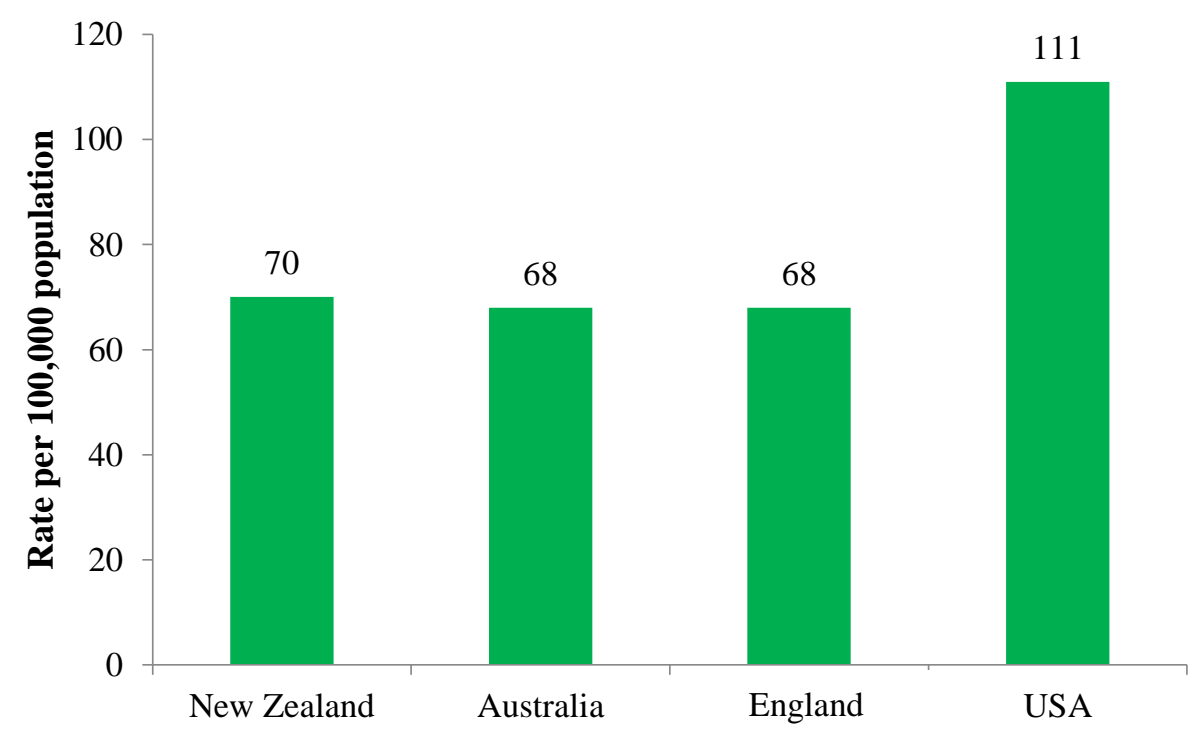

Figure 2.8. Rates of gonorrhoea per 100,000 population for four developed countries in 2014 
As incidence rates are dependent on testing rates, it may be that New Zealanders test more than people in other countries. For example, a cross-national study reported that although New Zealand appeared to have a higher rate of chlamydia than Denmark and Sweden from national incidence data, when the number of tests was considered, the total positive results per 100 tests was similar for the three countries (Bender et al., 2011). ESR considered testing rates in the 2014 STI annual surveillance report, and stated that New Zealand testing rates were higher than those reported in Australia but lower than testing rates for England (ESR Annual Surveillance Report 2014), suggesting that the higher rates of chlamydia in New Zealand are not entirely attributable to higher rates of testing. However, this is not a straightforward comparison, as the National Chlamydia Screening Programme (NCSP) in England may lead to larger numbers of lower risk individuals being tested than in New Zealand. It is also important to bear in mind that the methodology for calculating these rates varies by country.

Testing coverage rates were able to be calculated within the New Zealand surveillance data for the first time in 2014 in the high-risk age groups (15 to 29 years) by removing repeated tests for each unique individual (ESR Annual Surveillance Report 2014). This analysis indicated that less than $10 \%$ of males but between $23 \%$ and $38 \%$ of females in these age groups had at least one annual test. The report concluded that these testing rates were still almost all below the level mathematical modelling has suggested is required to decrease chlamydia prevalence (annual testing of $30 \%$ to $40 \%$ of all people under 25 years) (Regan et al., 2008, ESR Annual Surveillance Report 2014) indicating that more work needs to be done to encourage individuals to seek testing.

\subsubsection{Risk factors for STI}

Several factors are associated with a higher risk of contracting an STI. Some of these are personal characteristics (age, gender, ethnicity etc.) and some are behaviours. These factors are not distinct from each other; some risky behaviours are more common in certain populations, meaning there are complex relationships between risk factors which interact to determine a person's individual risk. 


\subsubsection{Populations most at risk of infection}

Age - Young people are disproportionately affected by STIs, with those under 25 years old typically having the highest rates of infection for many STIs excluding HIV and syphilis (Dielissen et al., 2013, Slater and Robinson, 2014). This trend is internationally consistent (Chinsembu, 2009, Detels et al., 2011, Dielissen et al., 2013). In the USA, the CDC STI Surveillance Report revealed that $65 \%$ of chlamydia cases and $53 \%$ of gonorrhoea cases in 2014 were among young people aged 15 to 24 (Centers for Disease Control and Prevention, 2015). A similar story is seen in the UK; the 2015 statistics from Public Health England (PHE) show that among heterosexuals diagnosed in SHCs, 62\% with chlamydia, 52\% with gonorrhoea, $51 \%$ with genital warts, and $41 \%$ with genital herpes were aged 15 to 24 years (Health Protection Report Volume 10 Number 22, 2016). The surveillance data from New Zealand illustrates the same trend, with the 15 to 24 year old age group also accounting for $67 \%$ of chlamydia and 57\% of gonorrhoea cases (ESR Annual Surveillance Report 2014).

This pattern is attributable to several factors. First, there are biological reasons that young females are more vulnerable to STIs than older women. In adolescence, the genital tract is not fully matured which means the vaginal epithelium and cervix have not begun to secrete mucous and the vaginal $\mathrm{pH}$ is high. Both of these factors reduce the vagina's defences against infection (Slater and Robinson, 2014). Cervical ectopy (where the favoured cell type of $C$. trachomatis serovars D to $\mathrm{K}$, the columnar epithelial cells, are exposed due to increased oestrogen) is also common in young females and has been shown to be associated with chlamydia infection (Lee et al., 2006). In addition, young people tend to demonstrate higher risk sexual behaviours. For example, they tend to change sexual partners more frequently (Slater and Robinson, 2014) and may be less well equipped to negotiate condom use than older, more experienced men and women. The combination of biological and sociological factors results in a higher risk of STI among young people. More detail about risky behaviours is included later in this section.

Gender - Overall, and especially at younger ages, women are far more likely to be diagnosed with an STI (Chinsembu, 2009, Detels et al., 2011, Dielissen et al., 2013). For example, in New Zealand there were more than twice the number of laboratory-diagnosed cases of chlamydia in females than in males in 2014 (ESR Annual Surveillance Report 2014). Again, 
this trend is seen all over the world (Detels et al., 2011) and is attributable to several reasons. Firstly, women are more susceptible than men to STIs due to their genital anatomy. The thin mucous membrane covering the vagina easily allows bacteria and viruses to pass compared to the thick skin of a penis (Anderson et al., 2014). The vagina is also an ideal environment for bacterial growth (Larsen and Monif, 2001). In addition, male ejaculate generally constitutes a higher volume than female secretions during sexual intercourse, meaning the amount of potentially infected material transmitted from partner to partner is larger from a male than from a female (Ottesen et al., 1987, Owen and Katz, 2005). Male ejaculate is also retained in the vagina for a period of time after unprotected non-withdrawal sex, whereas female secretions remain on the surface of the penis and are thus exposed to air which limits the survival of many STI organisms (Keeling and Rohani, 2008). It has also been suggested that hormonal changes during a woman's menstrual cycle may affect the local immune system in the female reproductive tract, making it easier for an organism to establish an infection (Venkatesh and Cu-Uvin, 2014, Wira et al., 2014).

It is possible that the higher rates of STI diagnoses observed in women may be partly attributable to higher rates of testing among women (Morgan and Haar, 2008, Cuffe et al., 2016). Screening programmes and opportunistic testing tend to target women because of the higher morbidity associated with STI in women, and also because of the perceived higher prevalence of infection (Low and Egger, 2002). It may also be partly down to women's increased willingness to obtain care generally, putting her in a position for opportunistic testing (Dickson et al., 1998, Low and Egger, 2002, Geisler et al., 2006). However, men are less likely to be asymptomatic when they have an STI (Malhotra et al., 2013, Davies et al., 2014a), meaning they are more likely to be aware of an infection if they have contracted one. Therefore it is unlikely that the higher rates seen in women are entirely due to higher rates of testing.

Ethnicity - Ethnicity appears to be associated with vulnerability to STIs: indigenous or marginalised ethnic groups typically experience the highest burden of infection within a country. This is seen in Australia where Indigenous (Aboriginal and Torres Strait Islander) people make up $2.5 \%$ of Australia's population, yet data from recent research studies suggests they account for $9 \%$ of chlamydia diagnoses each year (O'Connor et al., 2014). 
James Ward and colleagues have researched the incidence of STIs in Australian Indigenous communities (Silver et al., 2015), reported on discrepancy in positivity rates between Indigenous and non-Indigenous men and women (Graham et al., 2012, O'Connor et al., 2014), and investigated the correlates of STI diagnosis in Indigenous populations (Ward et al., 2016). This work has highlighted the disproportionate burden of disease for Indigenous people in Australia and has led to two randomised controlled trials (RCTs) to improve sexual health in Indigenous communities (Ward et al., 2013, Graham et al., 2015). The results of the first trial were promising, with the sexual health quality improvement program increasing rates of testing and positive diagnoses (Graham et al., 2015).

In the USA, the national rates among American Indians and Alaska Natives for chlamydia, gonorrhoea, and syphilis are two to six times the comparable rates for non-Hispanic white people (Winscott et al., 2010, Centers for Disease Control and Prevention, 2015). AfricanAmerican individuals have also been shown to be disproportionately affected (Centers for Disease Control and Prevention, 2015). Similarly, in Canada, rates of chlamydia, gonorrhoea and HIV are highest among Aboriginal populations (Steenbeek et al., 2006), although there appears to be a lack of sexual health research into the extent and determinants of this situation in Canada. In the UK, black Caribbean and black African adults have been shown to be more likely to receive an STI diagnosis (Fenton et al., 2005b, Gerressu et al., 2012).

The situation in New Zealand is much the same with Māori and Pacific peoples having higher rates of common STIs (Connor et al., 1997, Lo et al., 2002, Azariah and Perkins, 2007, Ekeroma et al., 2012, Rose et al., 2012, ESR Annual Surveillance Report 2014). In 2014, the highest estimated national rate of chlamydia in males was seen in the Māori ethnic group (735 per 100,000, 1933 cases), followed by Pacific peoples (695 per 100,000, 955 cases) and the Middle Eastern/Latin American/African group (501 per 100,000, 115 cases) (ESR Annual Surveillance Report 2014). The highest rate of chlamydia in females was also reported in the Māori ethnic group (2468 per 100,000, 6870 cases) followed by Pacific peoples (1953 per 100,000, 2738 cases) and European or Other (486 per 100,000, 7089 cases) ethnic groups (ESR Annual Surveillance Report 2014). This is an important issue, as inequities in health between indigenous New Zealanders and Pākehā (a term widely used in New Zealand to describe non-Māori/people from overseas, usually those of European 
descent) are common and may stem from and contribute to the ongoing harm of colonisation (Bramley et al., 2005, Baker et al., 2012). However, little research has been undertaken exploring the sexual health of these ethnic groups and as such, the reasons behind the discrepancy in incidence rates are unknown. Possible factors could include lack of equal access to services (Ward et al., 2013), sexual networking or socioeconomic factors. The latter is discussed in the following paragraphs.

Societal determinants - Sexual partnerships are formed in the context of social networks, so the social environment needs to be considered when studying sexual risk. Political, social and economic circumstances influence behaviour and thus STI risk of an individual. Previous studies have identified societal factors that have been connected to a heightened level of risky sexual behaviour and/or STI diagnosis, for example: poverty (Sales et al., 2014, Khan et al., 2015) and intimate partner or community violence (Brady, 2006, Senn et al., 2016).

On a general population level, inequities are evident, with the most marginalised of communities being the most vulnerable to STI acquisition (Aral, 2002). Internationally, low socioeconomic status has been shown to be associated with a higher risk of STI, as reviewed by Crichton et al. (2015). A study using laboratory data in an urban region of New Zealand linked to census records found that higher social deprivation as assessed by NZ Dep2001 Index of Deprivation was predictive of chlamydia infection (OR 1.66, 95\% CI $1.55-1.77$ ) (Rose et al., 2012). This suggests that the inequalities in STI risk reported internationally also occur within New Zealand.

Understanding the social determinants of STIs is vital to designing and implementing more effective prevention programs (Hogben and Leichliter, 2008). There is growing recognition that approaches which modify social norms, institutions, laws, policies and economics have the power to transform social structures and environments and reduce STI vulnerability (Gupta et al., 2008, Auerbach, 2009, Centers for Disease Control and Prevention, 2010, Sharpe et al., 2010). These approaches aim to address factors affecting individual behaviour, rather than targeting the behaviour itself (Centers for Disease Control and Prevention, 2010). Examples include the repeal of anti-sodomy laws in India to decriminalize homosexual behaviour, and the economic empowerment of women in sub-Saharan Africa to reduce dependence on men (Auerbach, 2009). In developed countries such as New Zealand, tackling 
poverty and social segregation is likely to have a significant impact on STI rates and disparities (Hogben and Leichliter, 2008).

\subsubsection{Risk behaviours}

In the context of this thesis, risky sexual behaviour is defined as behaviour related to sexual conduct that puts a person at increased risk of contracting or transmitting an STI. It has been demonstrated that people who display risky sexual behaviour are more likely to contract an STI than those with lower risk behaviour (Fenton et al., 2001b, Warner et al., 2006, Jorgensen et al., 2015, Scott et al., 2015). Risky sexual behaviours include having sex without a condom (Warner et al., 2006), having higher numbers of sexual partners (Fatusi and Wang, 2009, Scott et al., 2015), having partners in quick succession (Zhan et al., 2012, Jorgensen et al., 2015), or having concurrent sexual partners (defined as having two or more sexual partnerships that overlap in time) (Morris and Kretzschmar, 1995, Nelson et al., 2007).

Condoms protect individuals from STI acquisition by providing a barrier between the genitals of sexual partners that is impermeable to STI organisms. Condoms may fit over the penis (male condom) or be inserted into the vagina (female condom), although the latter are much less used. However, condoms do not cover the entire genital region meaning they are not as effective at preventing acquisition of STIs which are transmitted by skin to skin contact, such as genital herpes and genital warts (HPV). Attitudes towards condoms vary and research has found that many people dislike using condoms due to the perceived interference with sexual pleasure (Fennell, 2014, Higgins and Wang, 2015). Other reasons for inconsistent or no condom use may include lack of confidence in how to use condoms (Wong et al., 2013), or issues with negotiating use, especially in relationships with a significant power imbalance and intimate partner violence (Bergmann and Stockman, 2015, Nesoff et al., 2016).

Although condom non-use has often been shown to be associated with STI diagnosis in cross-sectional studies (Warner et al., 2006), the number of prospective studies assessing the effectiveness of condoms in preventing STIs is far fewer (Holmes et al., 2004). An expert panel was convened in 2000 to evaluate the peer-reviewed published evidence on the effectiveness of latex condoms in preventing STIs during vaginal intercourse (Hitchcock, 
2001). They concluded that consistent condom use reduced the risk of HIV transmission from men to women and women to men, as well as the risk of transmission of gonorrhoea from women to men. However, they found there was insufficient evidence from prospective studies to determine the effectiveness of condoms in preventing gonorrhoea and chlamydia from men to women or in preventing syphilis, chancroid, trichomoniasis, genital herpes or HPV infection in men or women. Following the subsequent publication of several prospective studies assessing condom efficiency, an updated review was undertaken (Holmes et al., 2004). The review found that condom use was associated with protection of men and women against gonorrhoea, chlamydia, syphilis and genital herpes, but there was no evidence that condoms were effective in preventing HPV. Limited data on the effectiveness of female condoms at preventing STI transmission are available, and studies have tended to focus on acceptability (Vijayakumar et al., 2006, Gallo et al., 2012).

STI acquisition is independently associated with increasing numbers of sexual partners (Fergusson et al., 2006). In Natsal 2 for example, only $0.6 \%$ of men and $1.8 \%$ of women with none or one partner during the past five years reported having been diagnosed with an STI, whereas $11.8 \%$ of men and $19.0 \%$ of women who reported having 10 or more partners during this period were diagnosed with an STI (Fenton et al., 2005a). However, it is likely that this is in part due to people with a larger number of sexual partners being more likely to attend for testing. In New Zealand, sera collected from participants in the Dunedin Multidisciplinary Health and Development Study were analysed for past $C$. trachomatis infection by Pgp3 (an immunodominant C. trachomatis antigen) antibody assay. Pgp3 antibody was correlated with increasing numbers of sexual partners, self-reported chlamydia infection, and younger age at sexual debut (Horner et al., 2016).

Temporal dimensions are important in STI epidemiology; gaps between partnerships and concurrency of partnerships are risk factors for the acquisition and transmission of STIs (Jorgensen et al., 2015). Gap length is an important determinant because if the gap between partnerships for an infected individual is shorter than the remaining infectious period, there is possibility of transmission to the new partner. A study of 2,203 genitourinary medicine (GUM) clinic patients in England in 2009 found that the median number of days between partners were short at just 14 and 24 days for men and women respectively (Mercer et al., 
2013b). In over half of the gaps, condoms were used inconsistently with one or both partners, and in one-quarter, condoms were never used with either partner. Short gap lengths have been found to be associated with STI diagnoses in various populations (Kraut-Becher and Aral, 2003, Zhan et al., 2012, Jorgensen et al., 2015).

Although STI acquisition can occur in the context of serial monogamy, earlier partners in the sequence are not exposed to infections that the index case acquires from subsequent partners. In contrast, with concurrent partnerships, all partners may be exposed to an infection acquired from any partner, therefore concurrency of sexual partnerships is a significant driver of STI spread (Morris and Kretzschmar, 1995, Morgan, 2013). This has been observed in several studies, for example, in a recent Danish cross-sectional study a strong association was found between concurrent partnerships $(\mathrm{OR}=12.5,95 \% \mathrm{CI}=7.7-20.4)$ and having a verified $C$. trachomatis infection (Jorgensen et al., 2015).

In addition, the sexual orientation behaviour of an individual affects their risk of acquiring an STI. The term 'sexual orientation behaviour' is used, rather than 'sexuality', because sexuality is a term used to describe a wide range of factors related to sex, not just to refer to which gender(s) an individual has sex with. In addition, it is the behaviour which is important in this context, as it is behaviour, not self-identity, which is related to STI risk, and the two may not always correspond (Smith et al., 2003b). It is well documented that MSM are more at risk of STIs than men who only have sex with women (MSW) (Fenton et al., 2005a, Mayer, 2011, NZSHS, 2015d). This is due to several factors. Biologically, the thin anorectal muscosal surface is highly susceptible to infection (Janeway et al., 2001, Hughes and Saxton, 2015). MSM tend to have longer periods of partnership acquisition, higher numbers of sexual partners and a higher prevalence of partnership concurrency (Glick et al., 2012).

Sociologically, homophobia and stigmatisation may discourage stable relationships or increase stress on relationships, which may in turn lead to more casual, anonymous and opportunistic contacts (Doyle and Molix, 2015). In addition, there is no contraceptive reason to use barrier protection for same-sex partnerships, which may result in lower condom use.

\subsubsection{Factors associated with high risk behaviours}

There is a large body of research investigating the association between alcohol use and sexual risk behaviour (Weinhardt and Carey, 2000, Cooper, 2002, Shuper et al., 2010, Boden 
et al., 2011, Deogan et al., 2012, Rehm et al., 2012, Connor et al., 2013). Data from Natsal 1 and 2 demonstrate an association between heavy drinking and higher number of sexual partners, as well as with having unprotected sex with two or more partners in the past year (Aicken et al., 2011b). Similarly, a population-based study of 100,790 14 to 16 years olds in Finland reported that the likelihood of engaging in sexual intercourse, having unprotected sex and/or having multiple sexual partners increased with the frequency of alcohol use and the frequency of drunkenness-related drinking (Lavikainen et al., 2009). In New Zealand, a study of 2,921 students from across six universities found that the reported number of partners in the past 12 months was related to heavy alcohol use as assessed by the first three questions of the Alcohol Use Disorders Identification Test (AUDIT-C) questionnaire (Connor et al., 2013). However, condom use at last sex was not associated with AUDIT-C scores. Research has tended to focus on adolescents (Lavikainen et al., 2009, Deogan et al., 2012, Snipes and Benotsch, 2013), but there is evidence that this relationship occurs in older ages too (Connor et al., 2015).

As risk behaviours tend to cluster (Meader et al., 2016) it is difficult to untangle whether alcohol use directly causes individuals to engage in high-risk sexual behaviours through pharmacological effects, through expectations about how alcohol affects sexual behaviour (self-fulfilling prophecies), or whether there is confounding due to personality factors such as compulsivity, risk-taking or sensation seeking (Shuper et al., 2010, Rehm et al., 2012, Connor et al., 2013). Risky sexual behaviour puts individuals at risk for acquiring STIs and there is evidence that alcohol use is associated with STI and HIV acquisition (Cook and Clark, 2005, Baliunas et al., 2010). A New Zealand study used longitudinal data from the Christchurch Health and Development Study (CHDS) to investigate the possible underlying cause (Boden et al., 2011). The authors used conditional fixed-effects regression models to control for the effects of non-observed fixed confounding factors that may be correlated with both alcohol use and STI diagnosis, and found that an association between alcohol use and STI diagnosis remained. This lends support to the argument that the link between alcohol and STI diagnosis is due to pharmacological effects rather than underlying personality factors such as compulsivity, risk-taking or sensation seeking. Some studies have taken an RCT approach, by randomising alcohol consumption and measuring the impact on the selfperceived intent to engage in unprotected sex. A systematic review of this type of evidence 
was undertaken by Rehm et al. (2012), which concluded that blood alcohol concentration is an independent risk factor for the intention to engage in unprotected sex. However, the review only assessed evidence from studies of young adults in North American college and community settings, making the generalisability of results to other age groups and populations uncertain.

Illicit drug use has also been associated with sexual risk behaviour and STI diagnosis (Boyer et al., 1999, Forrest et al., 2010, Spauwen et al., 2015, Hegazi et al., 2016), but like alcohol, the causality in this relationship has been difficult to establish (Melendez-Torres and Bourne, 2016). Sex while using recreational drugs use may be coincidental, or recreational drugs may be intentionally combined with sex in order to facilitate or enhance the sexual encounter (Bourne et al., 2015). The latter has been termed 'chemsex'. Research in many countries suggests that MSM use stimulant drugs more commonly than non-MSM and that use is increasing, especially with the rise in popularity of substances such as gammahydroxybutyric acid (GHB), gamma-butyrolactone (GBL), and crystallised methamphetamine (Office for National Statistics, 2014, Bourne et al., 2015). Although prevalence of this behaviour is still relatively low, especially outside of urban areas, it has serious implications for both sexual and general health (McCall et al., 2015, Hegazi et al., 2016).

\subsubsection{Network dynamics}

In addition to personal sexual risk behaviours, sexual partners' behaviours are also critical determinants of exposure to infection. For example, the number of previous and concurrent partners a sexual partner has, and the risk status of those partners, also matters. In this way, the sexual risk of an individual is affected by the network within which their sexual partnerships place them (see Figure 2.9). 


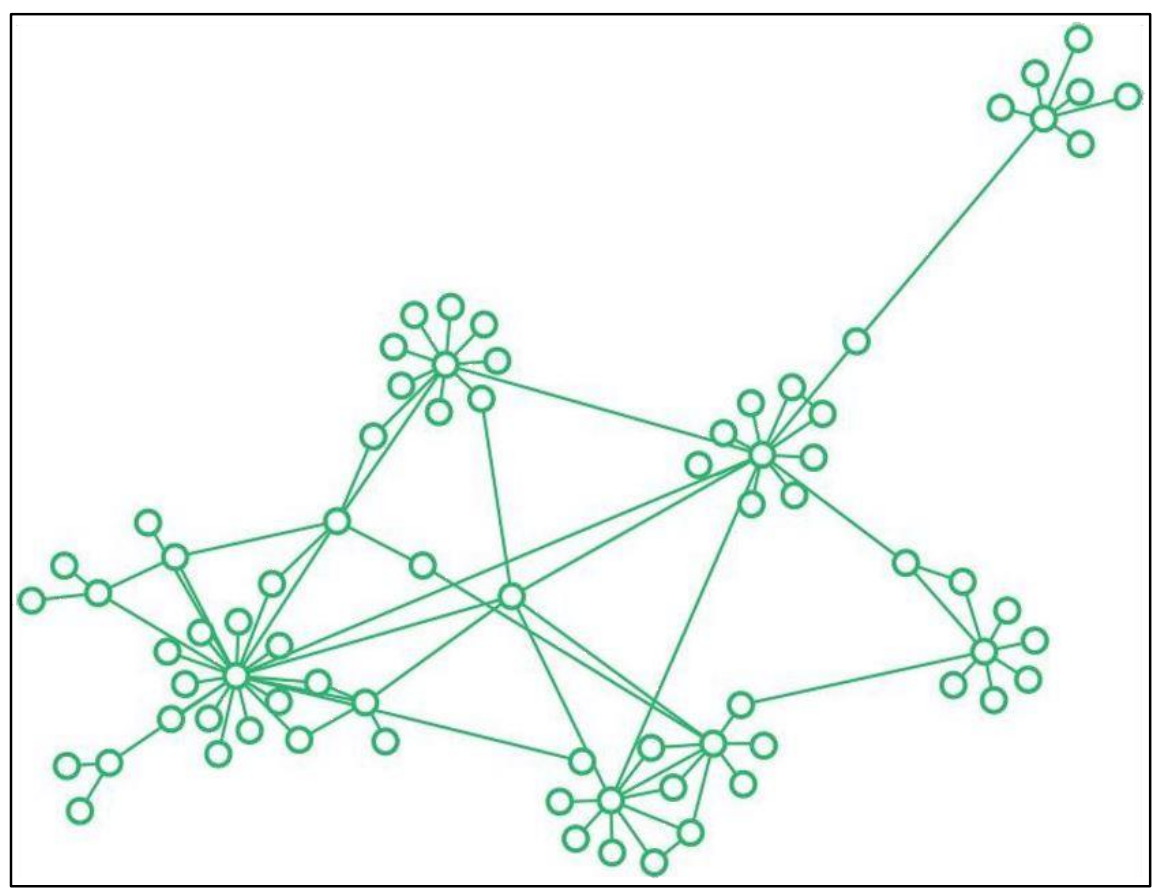

Figure 2.9. Example of a sexual network Reproduced with permission from $\mathbf{N}$ Masuda and P Holme.

Social network research approaches have demonstrated the importance of 'core groups' in the spread of STIs (Yorke et al., 1978, Aral, 2002). Core groups are sub-groups of the population that are small in size but have high STI rates and contribute disproportionately to the transmission of STIs, although definitions vary (Thomas and Tucker, 1996, Gesink et al., 2011). Individuals in core groups tend to have large numbers of sexual partners, therefore STIs are transmitted more often and become endemic within the core group more quickly (Jolly et al., 2001). People who have sex with members of core groups and members of the general population serve as a 'bridge population' and facilitate the spread of STIs from core groups to the general population and vice versa (Mercer et al., 2009). Examples of core groups include drug users, truck drivers and MSM (Aral, 2006), although a definition based on increased transmission of infection includes anyone with a high number of sexual partners within a given time period (Humblet et al., 2003). Understanding the networking of members of core groups and bridge populations is important because it has key implications for infection control and management. Due to individuals' changes in sexual behaviour over time, core groups have a constantly changing membership, adding to the complexity of this objective (Stigum et al., 1997, Humblet et al., 2003). 
Another feature of network dynamics is the pattern of mixing which individuals demonstrate. Assortative mixing is where individuals form sexual partnerships with individuals of similar risk status. The converse pattern is disassortative mixing, where individuals form sexual partnerships with individuals of dissimilar risk status. This factor may explain some of the discrepancies in STI rates between ethnicities, as introduced earlier in this section. Studies from the USA have demonstrated that African Americans are more likely to choose sexual partners with dissimilar risk status than white people, who exhibit more assortative sexual mixing (Laumann and Youm, 1999). However African Americans also demonstrate assortative mixing in terms of limiting sexual partnerships from outside of that ethnic group. This then leads to high rates of STI transmission limited to within the African American network.

\subsection{Factors that influence the reproductive rate of infection}

This section introduces the epidemiological model of the basic reproductive number, including descriptions of the various parameters. It explains why reducing the duration of infection is a key aspect of STI control and the focus of this thesis.

\subsubsection{Basic reproductive number}

The rate that an infection spreads in a population can be understood using an epidemiological model of the basic reproductive number, known as $\mathrm{R}_{0}$ (Macdonald, 1952, Garnett, 2002, Smith et al., 2012). The basic reproductive number is essentially the number of secondary cases which a single infectious individual case will produce in a completely susceptible population (Dietz, 1993). The standard model defines $\mathrm{R}_{0}$ as a product of the efficiency of transmission $(\beta)$, the average number of new sexual partners in the population $(c)$, and the average duration of infectiousness $(D)$ :

$$
\mathrm{R}_{0}=\beta c D
$$

If $\mathrm{R}_{0}$ is less than 1 , each existing infection will cause on average less than one new infection, meaning the number of new infections will decline until disappearance of the organism (Dietz, 1993). $\mathrm{R}_{0}$ is not a fixed number for any infection, as it can change depending on 
human behaviour (Dietz, 1993). Epidemiological and public health interventions targeting human behaviour are essentially attempting to alter the factors in the equation in order to cause $\mathrm{R}_{0}$ to be less than 1 .

This formulation of $\mathrm{R}_{0}$ is based on a hypothetical, randomly mixed homogeneous population. However, real mixing patterns are likely to be more complex due to variations in host behaviour such as assortative and disasortative mixing, as discussed in section 2.3.2.4. (Siettos and Russo, 2013). These and other factors will affect $\mathrm{R}_{0}$, therefore this equation must be viewed as a simplified model. However, it is an appropriate starting point to understand transmission dynamics and where to focus control efforts. The various factors in the equation are discussed in the following subsections.

\subsubsection{Transmissibility of infection ( $\beta$ )}

The efficiency of transmission is essentially the probability of transmission of infection from an infected individual to a susceptible person. There must be an exchange of body fluids in order to transmit most STIs, although some organisms such as HSV and HPV can be transmitted by skin-to-skin contact (World Health Organization, 2013, Hutter and Decker, 2016, Koren and Decker, 2016). This is because many STIs do not survive for long outside of the human body (Keeling and Rohani, 2008). It is also possible for some STIs to be transmitted from a pregnant woman to her unborn child during vaginal delivery (vertical transmission), and for blood-borne STIs to be transmitted to infants through breast milk or from person to person through the sharing of needles or needle stick injuries (Bartlett, 1993, World Health Organization, 2013). However, this thesis is concerned with testing for infections that have been acquired sexually, so these alternative transmission routes will not be discussed.

Transmissibility rates through sexual intercourse have been estimated for a range of STIs using data from long-term couples (Lycke et al., 1980, Hernandez et al., 2008, Hochberg et al., 2015) and from modelling studies (Davies et al., 2014a). However, there are no reliable studies of transmission rates following single episodes of sexual exposure for many STIs other than HSV (Stanaway et al., 2012) and HIV (Boily et al., 2009, Baggaley et al., 2010). What we can conclude from the studies that have been done is that transmission tends to be more efficient from the penis to the vagina or rectum rather than vice versa for infections that 
require the exchange of body fluids (due to retention of infected ejaculate) (Aral, 2006). It can also be concluded that the probability of transmission is much higher for bacterial STIs than for other STIs, and gonorrhoea is more infective than chlamydia (Aral, 2006).

The transmissibility of an STI may also be influenced by co-infection with other STIs. The high co-infection rates observed (Connor et al., 1997, Ward and Ronn, 2010, Mlisana et al., 2012, Li et al., 2016) are partly due to continuous practice of sexual risk behaviours, but there is also evidence of biological influences, as previously discussed in section 2.2.3.

\subsubsection{Number of sexual contacts (c)}

It is intuitive that the more partners an individual has while they are infected with an STI, the more people they can potentially pass the infection on to. Highly sexually-active members of the population contribute disproportionately to the spread of infection because they are more at risk for both contracting and transmitting infections (Ashby and Gupta, 2013). However, the number of sex acts is also important. Mathematical modelling has suggested that individuals who have a just a few sexual partners but a large number of sex acts per partner may also play a significant role in the transmission of STIs (Nordvik and Liljeros, 2006). In addition, the number of sex acts may not be constant across sexual partners. If the number of sex acts per sexual partner is not constant, each contact between susceptible and infected individuals does not have the same transmission probability, so affects the overall rate of transmission within the population.

The most recent national sexual behaviour survey in the UK, Natsal 3, found that the median lifetime number of sexual partners for the whole study population $(\mathrm{n}=15,162)$ was six (Mercer et al., 2013c). There has not been a national survey of sexual behaviour in New Zealand since 1991 (Paul et al., 1995), so the sexual behaviour of New Zealand's general population is not known. However, a 2009 survey of students across six New Zealand universities found that the median lifetime number of sexual partners among New Zealand university students was three (Psutka et al., 2012). This aligned with the data from the 16 to 24 year old age-group in Natsal 3 (median =3). However, aggregating the data is not particularly useful as there will be large variation in the number of partners within the population. The New Zealand university study found that although the median number of partners in the past 12 months was just one, around $25 \%$ of the students reported three or 
more partners during this time (Psutka et al., 2012). Interestingly, the study found that the use of a condom at last sex became less likely as the number of sexual partners increased $(p=$ 0.042). Of particular importance is the observation that MSM typically have higher numbers of partners than other sexual preference groups, which contributes to their increased risk for STI (Levin et al., 2009, Glick et al., 2012).

\subsubsection{Duration of infection (D)}

The length of time between infection and treatment or natural clearance determines the duration of infectiousness (Figure 2.10). When a pathogen enters a host, this is termed the 'exposure moment'. The pathogen moves to the preferred tissues or cells after entering the body and will begin multiplying or replicating; at this stage it is considered an infection. The period between exposure and infectiousness is termed the 'latent period' (note this is different to 'clinical latency', a term used to describe when a virus is subclinical but may be transmitted), and the period between exposure and the onset of clinical signs and symptoms is termed the 'incubation period' (see Table 2.1 for the incubation periods of some common STIs) (Gail and Benichou, 2000). A host may become infectious with or without the presence of symptoms.

\section{Diagnosis and treatment effective}

or

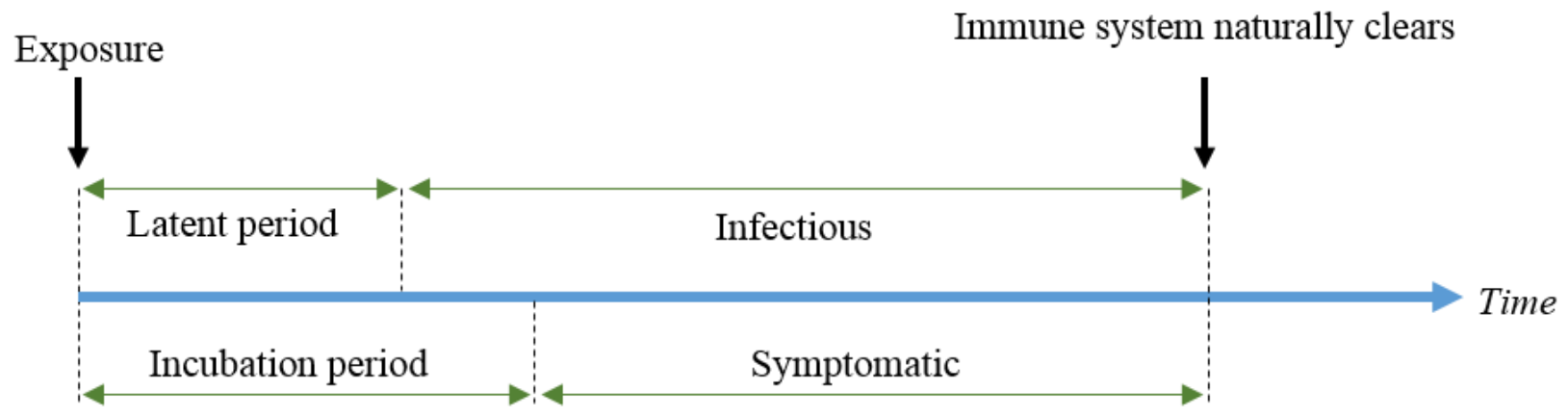

Figure 2.10. Duration of an infection 
It is difficult to assess the natural history of infection in humans due to the ethical implications of leaving a treatable condition untreated. Assessing the natural history of an STI was the objective of the now infamous Tuskegee syphilis study in the USA, where hundreds of men were left untreated and uninformed about their infection status for up to 40 years (White, 2000). This led to the creation of the National Research Act (1974) in the United States and ultimately the scientific practice of requiring prior approval for human studies from ethical review boards (Centers for Disease Control and Prevention, 2013b). That said, Morre et al. (2002) prospectively followed 30 asymptomatic $C$. trachomatis-positive women for one year and found the person/time clearance rate of infection was $4.9 \%$ per month. Another study assessed Danish male recruits at enrolment for C. trachomatis infection and then followed them up five to eight months later (van den Brule et al., 2002). Of the nine men with a baseline positive sample who had not been treated for the infection, only one had cleared the infection. Molano et al. (2005) retrospectively used clinical specimens and questionnaire data from a study examining the natural course of HPV infection for a study of the natural history of $C$. trachomatis. Women whose sample from the first visit was positive for $C$. trachomatis and who were asymptomatic were included in the analysis $(\mathrm{n}=82)$, and the results from their samples taken at follow-up visits used to determine length of infection (median time of follow-up was 5.7 years). Approximately $46 \%$ of the infections were persistent at one year, $18 \%$ at two years, and $6 \%$ at four years of follow-up. However, the infection status of the women's sexual partners was not known, meaning that repeated infection from an untreated sexual partner might have biased the results in such a way that the duration of infection was increased.

Some studies have derived duration of infection from the prevalence of an infection divided by the incidence within the same population or region. This was the method used by Chow et al. (2016) in their systematic review of the duration of gonorrhoea and chlamydia infection among MSM. They found that gonorrhoea had a shorter duration of infection (114-138 days at the pharynx and 346 days at the rectum) than chlamydia (667 days at the pharynx and 579 days at the rectum).

As established in the epidemiological model of the basic reproductive number $\left(\mathrm{R}_{0}\right)$, the duration of infectiousness is an important component of transmission dynamics. Therefore, 
reducing the duration of infectiousness is a key goal of STI control efforts. One way to do this is to reduce the time between infection and treatment by encouraging individuals to seek STI testing in a timely manner so that any infection may be diagnosed and treated accordingly. This is important not only for curable STIs, but also for incurable viral STIs such as HSV and HIV, as antiviral therapies can reduce the pathogenicity of an infection by reducing viral load, thus reducing duration of infectiousness (Pau and George, 2014).

\subsection{Healthcare-seeking behaviour for STI testing and the factors that may influence it}

In this section, healthcare-seeking behaviour is defined and discussed with a focus on delay behaviour. Both quantitative and qualitative literature is reviewed to identify what is known about healthcare-seeking behaviour for STI and where further research is required. Factors that may influence healthcare-seeking behaviour are highlighted, including STI knowledge.

\subsubsection{Healthcare-seeking behaviour}

There are many definitions of healthcare-seeking behaviour in the literature (Fortenberry, 1997, Cornally and McCarthy, 2011, Ingabire et al., 2016). For the purposes of this thesis, healthcare-seeking behaviour is defined as the series of actions that people adopt to access and utilise formal healthcare in relation to their health concerns. The term 'healthcareseeking behaviour' is used to focus on the utilisation of the healthcare system. This is distinct from the more general term 'health-seeking behaviour' which has been used to describe how people make 'healthy choices' in their lifestyle behaviours, which may include their use of medical care and treatment (MacKian, 2001). The latter has its roots in psychology, and many social cognition models have been developed to predict possible behaviour patterns. One of the most widely applied is the 'health belief model' (Rosenstock, 1974), other approaches have included the 'theory of planned behaviour' (Ajzen, 1991). These models have been used to demonstrate the complexity of influences on an individual's behaviour at a given time and place. However, they have also been criticised for their inherent assumptions that humans are rational decision-makers who systematically review available information 
and form behaviour intentions from the information they receive (MacKian, 2001, Sniehotta et al., 2014). They also focus on the individual rather than take into account the social environment (Cochran and Mays, 1993). While some of these models may offer insight into healthcare-seeking for STI testing, their use is beyond the scope of this thesis which is focused on quantifying the temporal dimension of healthcare-seeking behaviour for STI testing and uncovering factors associated with action or delay.

Healthcare-seeking is a complex research topic, and there are additional complexities associated with STIs as they are stigmatised and sensitive conditions (Mapp et al., 2016). A better understanding of STI-related healthcare-seeking behaviour has been recognised as a key objective in STI control by major health funders and organisations. For example, the WHO has listed healthcare-seeking behaviour as an area that requires enhanced research to help improve service delivery in the draft Global Strategy for STIs 2016-2021 (under Strategic Direction 5, innovation for acceleration) (World Health Organization, 2016a). The Strategy stresses the need to engage individuals in care as early as possible.

\subsubsection{Time to presentation}

It should not be assumed that those with symptoms will seek healthcare immediately. Delay behaviour refers to the time spent between perceiving physical symptoms potentially attributable to an STI and health care utilisation (Leenaars, 1993). For an individual, early diagnosis of STIs is the best opportunity for effective medical treatment and support. At a population level, the importance of delay behaviour in terms of disease control depends on what additional potential for STI transmission is generated by delay. If an infected person has unprotected sex with a new partner, there is a risk they will pass on the STI. A study in UK GUM clinics found that around half of patients surveyed had continued to engage in sexual activity before attending clinic, despite being symptomatic (Mercer et al., 2007). A total of $4.2 \%$ of the sample reported unprotected sex with at least one new partner since symptoms had begun. A retrospective review of GUM clinic case notes by Sanmani et al. (2008), also in the UK, found that none of the symptomatic patients in their study reported unprotected sex with a new partner after booking their appointment, although it is possible that social desirability bias (the tendency of respondents to answer questions in a manner that will be viewed favourably by others) may have influenced results in this study. 
Previous literature assessing time to presentation for STI symptoms is summarised in Table 2.3. Some studies reported the median length of time to presentation, some the proportion of people who delayed, and some studies reported both. The majority of studies defined delay as waiting longer than 7 days to seek healthcare after the onset of symptoms. The median length of time to presentation varied considerably between studies, ranging from 3 days (Mercer et al., 2012) to 30 (Pitts et al., 2000, Thi Thu et al., 2007) days, although studies were from a range of populations so cultural and social factors would have been different. The studies are also from different years, with the earliest being published in 1993 (Leenaars, 1993, Sawyer and Moss, 1993) and the latest in 2013 (Malek et al., 2013, Mercer et al., 2013a). Despite these differences, there are some key messages that can be derived. Firstly, delay in seeking healthcare for STI symptoms is common in many countries. Secondly, many people (typically $30-60 \%$ ) continue to engage in sexual activity after the onset of symptoms. It also appears that more women than men have sex while symptomatic. In addition, in studies which found a gender difference in length of delay, women typically waited longer to seek healthcare than men (Leenaars, 1993, Moses et al., 1994, Choi et al., 1999). Even though several studies investigated whether participants continued to be sexually active while symptomatic, very few studies statistically analysed the association between delay behaviour and sex while symptomatic. Two studies found that people who delayed seeking care were more likely to have sex while experiencing symptoms (Moses et al., 1994, Thi Thu et al., 2007). Conversely, Irwin et al. (1999) found that attending healthcare quicker was associated with sexual activity while symptomatic.

Many studies have looked at factors associated with delayed healthcare-seeking, with contradictory results. For example, Meyer-Weitz et al. (2000), Liu et al. (2002) and Thi Thu et al. (2007) found that those with a lower level of education were more likely to delay seeking healthcare for symptoms, whereas Hook et al. (1997) found those with a lower education were actually less likely to delay, and Irwin et al. (1997) and Leenaars (1993) found no association between education level and delay behaviour. Contradictory associations were also found for age, ethnicity, employment status, income and whether an individual had had an STI before (See Table 2.3). 
Lastly, the literature review summarised in Table 2.3 shows that time to presentation for STI symptoms has not been assessed in New Zealand other than a small study $(n=66)$ of patients with genital warts at the Auckland Sexual Health Service (Ireland et al., 2005). This study did not assess whether patients continued to have sex while symptomatic. Therefore, it is not known if New Zealanders delay seeking healthcare for more general STI symptoms and what the risk for onwards transmission is during this time period. 
Table 2.3. Published studies assessing time to presentation for STI symptoms

\begin{tabular}{|c|c|c|c|c|c|c|c|c|}
\hline $\begin{array}{l}\text { First author } \\
\text { (year) }\end{array}$ & Country & $\begin{array}{l}\text { Study } \\
\text { population }\end{array}$ & Study size & $\begin{array}{l}\text { Response } \\
\text { rate }\end{array}$ & $\begin{array}{l}\text { Delay in } \\
\text { seeking } \\
\text { healthcare }\end{array}$ & $\begin{array}{l}\text { Predictors of, or } \\
\text { associations with, delayed } \\
\text { healthcare-seeking }\end{array}$ & Transmission risk & $\begin{array}{l}\text { Transmission } \\
\text { risk in } \\
\text { relation to } \\
\text { time to } \\
\text { presentation }\end{array}$ \\
\hline $\begin{array}{l}\text { Akinnawo } \\
\text { (1997) }\end{array}$ & Nigeria & $\begin{array}{l}\text { STI patients } \\
\text { selected from } \\
\text { urban health } \\
\text { institutions }\end{array}$ & 41 & $\begin{array}{l}\text { Information } \\
\text { not } \\
\text { provided }\end{array}$ & $\begin{array}{l}19.5 \% \\
\text { delayed } \\
\text { seeking care } \\
\text { for }>7 \text { days }\end{array}$ & Not assessed & Not assessed & Not assessed \\
\hline $\begin{array}{l}\text { Cassell } \\
(2003)\end{array}$ & UK & $\begin{array}{l}\text { New patients at } \\
\text { an outer } \\
\text { London GUM } \\
\text { clinic }\end{array}$ & 721 & $\begin{array}{l}752 / 1084 \\
(69.4 \%)\end{array}$ & $\begin{array}{l}\text { Median of } 7 \\
\text { days }\end{array}$ & $\begin{array}{l}\text { This paper focused on } \\
\text { whether attendees had } \\
\text { previously visited their GP. } \\
\text { Those who had seen their } \\
\text { GP reported a longer } \\
\text { duration of symptoms than } \\
\text { those attending GUM } \\
\text { directly }\end{array}$ & Not assessed & Not assessed \\
\hline $\begin{array}{l}\text { Choi } \\
(1999)\end{array}$ & China & $\begin{array}{l}\text { Symptomatic } \\
\text { STI patients } \\
\text { attending STI } \\
\text { clinics in } \\
\text { Guangzhou and } \\
\text { Shenzhen }\end{array}$ & 939 & $\begin{array}{l}\text { Information } \\
\text { not } \\
\text { provided }\end{array}$ & $\begin{array}{l}27 \% \text { delayed } \\
\text { seeking care } \\
\text { for }>2 \text { weeks }\end{array}$ & $\begin{array}{l}\text { Women were more likely } \\
\text { to delay than men. Among } \\
\text { men, those previously } \\
\text { seeking treatment, } \\
\text { experiencing no urethral } \\
\text { discharge, or paying for } \\
\text { sex were more likely to } \\
\text { delay }\end{array}$ & Not assessed & Not assessed \\
\hline $\begin{array}{l}\text { Faxelid } \\
(1994)\end{array}$ & Zambia & $\begin{array}{l}\text { Patients with an } \\
\text { STI attending } \\
\text { two outpatient } \\
\text { clinics }\end{array}$ & 100 & $\begin{array}{l}100 / 103 \\
(97.1 \%)\end{array}$ & $\begin{array}{l}\text { Median of } 14 \\
\text { days for } \\
\text { women, } 5 \\
\text { days for men }\end{array}$ & $\begin{array}{l}\text { No difference in time to } \\
\text { presentation between those } \\
\text { who knew and did not } \\
\text { know STI-related } \\
\text { symptoms }\end{array}$ & Not assessed & Not assessed \\
\hline
\end{tabular}




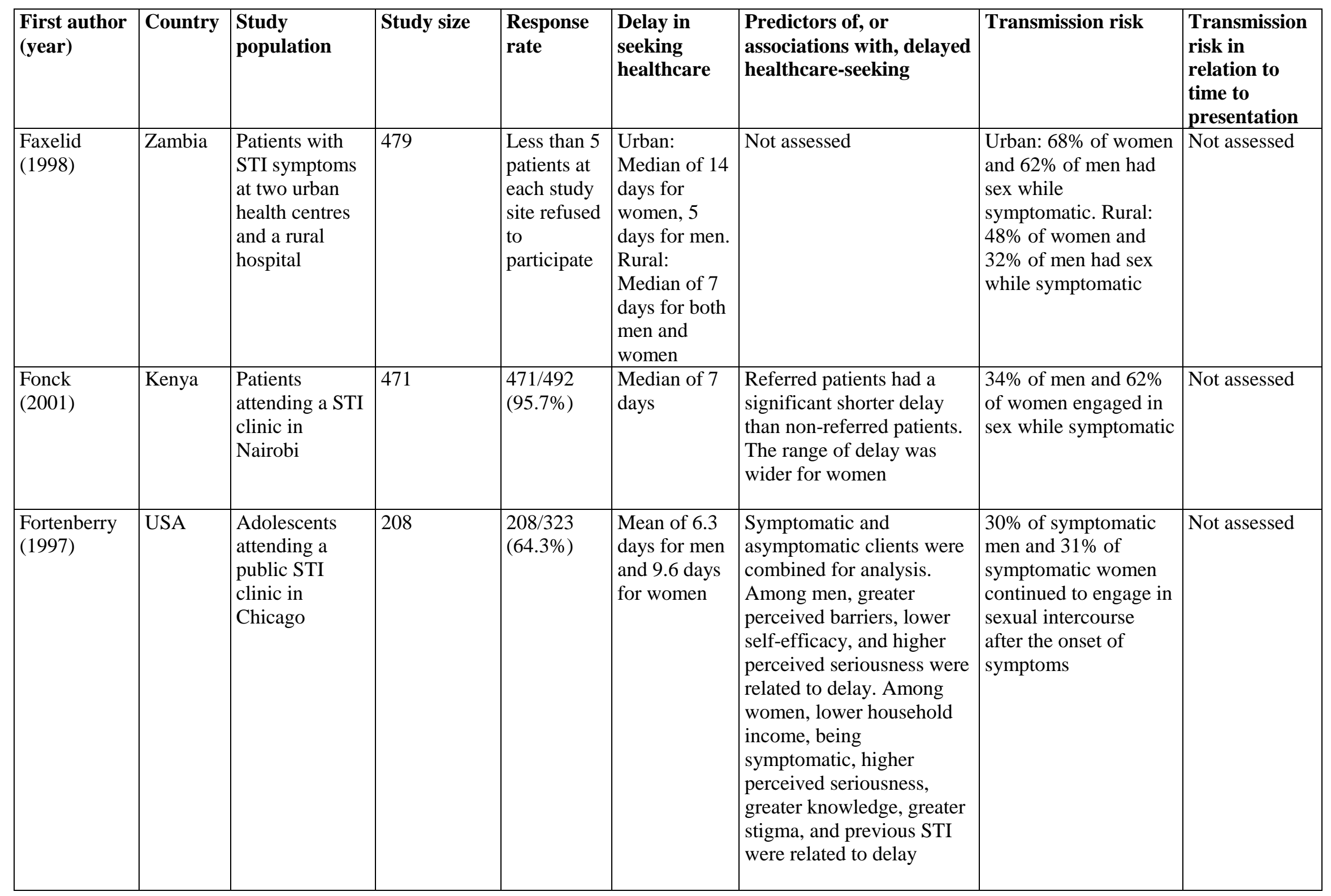




\begin{tabular}{|c|c|c|c|c|c|c|c|c|}
\hline $\begin{array}{l}\text { First author } \\
\text { (year) }\end{array}$ & Country & $\begin{array}{l}\text { Study } \\
\text { population }\end{array}$ & Study size & $\begin{array}{l}\text { Response } \\
\text { rate }\end{array}$ & $\begin{array}{l}\text { Delay in } \\
\text { seeking } \\
\text { healthcare }\end{array}$ & $\begin{array}{l}\text { Predictors of, or } \\
\text { associations with, delayed } \\
\text { healthcare-seeking }\end{array}$ & Transmission risk & $\begin{array}{l}\text { Transmission } \\
\text { risk in } \\
\text { relation to } \\
\text { time to } \\
\text { presentation }\end{array}$ \\
\hline $\begin{array}{l}\text { Gott } \\
(1999)\end{array}$ & UK & $\begin{array}{l}\text { New clinic } \\
\text { attendees aged } \\
50 \text { years or over } \\
\text { at three clinics } \\
\text { in England }\end{array}$ & 224 & $\begin{array}{l}224 / 274 \\
(81.7 \%)\end{array}$ & $\begin{array}{l}43.8 \% \text { waited } \\
>2 \text { weeks }\end{array}$ & $\begin{array}{l}\text { Those who delayed were } \\
\text { more likely to have tested } \\
\text { for HIV and to report they } \\
\text { had received enough } \\
\text { information about STIs. } \\
\text { Age was associated with } \\
\text { delay behaviour (those }>60 \\
\text { yrs more likely to delay for } \\
>2 \text { weeks). No associations } \\
\text { with gender, STI diagnosis, } \\
\text { marital status or previous } \\
\text { attendance }\end{array}$ & Not assessed & Not assessed \\
\hline $\begin{array}{l}\text { Hook } \\
(1997)\end{array}$ & USA & $\begin{array}{l}\text { Clients } \\
\text { attending five } \\
\text { publically } \\
\text { funded urban } \\
\text { STI clinics in } \\
\text { America }\end{array}$ & $\begin{array}{l}2590(1621 \\
\text { with } \\
\text { symptoms })\end{array}$ & $\begin{array}{l}\text { Approx. } \\
15 \% \text { of all } \\
\text { patients } \\
\text { attending } \\
\text { clinics }\end{array}$ & $\begin{array}{l}\text { Median of } 5 \\
\text { days for men } \\
\text { and } 7 \text { days } \\
\text { for women }\end{array}$ & $\begin{array}{l}\text { In men, those }>34 \text { yrs, of } \\
\text { black ethnicity, or with } \\
\text { lower education were less } \\
\text { likely to delay. In women, } \\
\text { those who were of white or } \\
\text { Filipino/Pacific ethnicity } \\
\text { were more likely to delay, } \\
\text { and black women less } \\
\text { likely. In both men and } \\
\text { women, delay was } \\
\text { associated with STI } \\
\text { organism }\end{array}$ & Not assessed & Not assessed \\
\hline $\begin{array}{l}\text { Ireland } \\
(2005)\end{array}$ & $\begin{array}{l}\text { New } \\
\text { Zealand }\end{array}$ & $\begin{array}{l}\text { Patients being } \\
\text { treated for } \\
\text { genital warts at } \\
\text { the Auckland } \\
\text { Sexual Health } \\
\text { Service }\end{array}$ & 66 & $\begin{array}{l}\text { Information } \\
\text { not } \\
\text { provided }\end{array}$ & $\begin{array}{l}61 \% \text { sought } \\
\text { treatment } \\
\text { within } 6 \\
\text { weeks, } 13 \% \\
\text { delayed for } \\
>1 \text { year }\end{array}$ & $\begin{array}{l}\text { Depression was associated } \\
\text { with longer delay }\end{array}$ & Not assessed & Not assessed \\
\hline
\end{tabular}




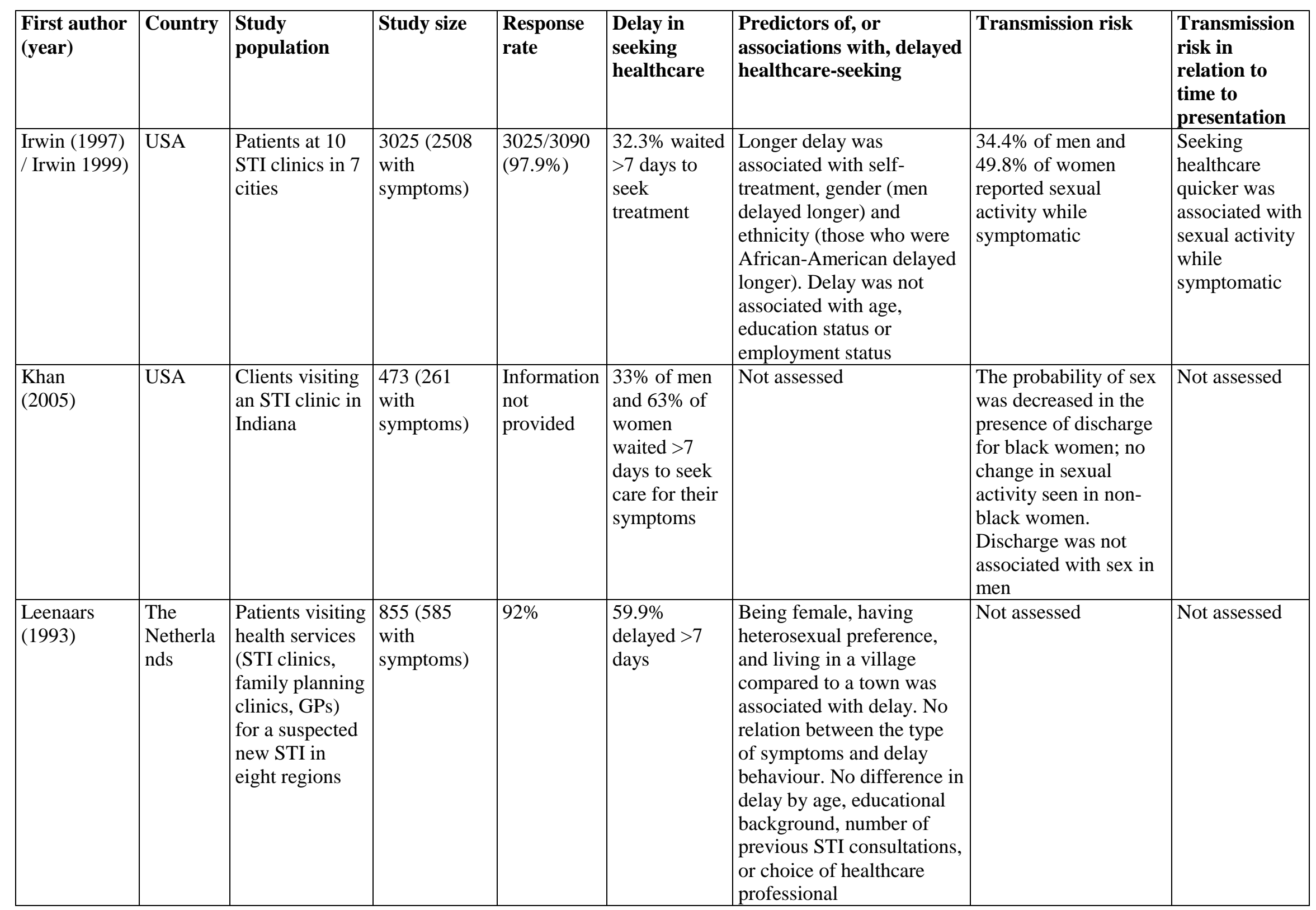




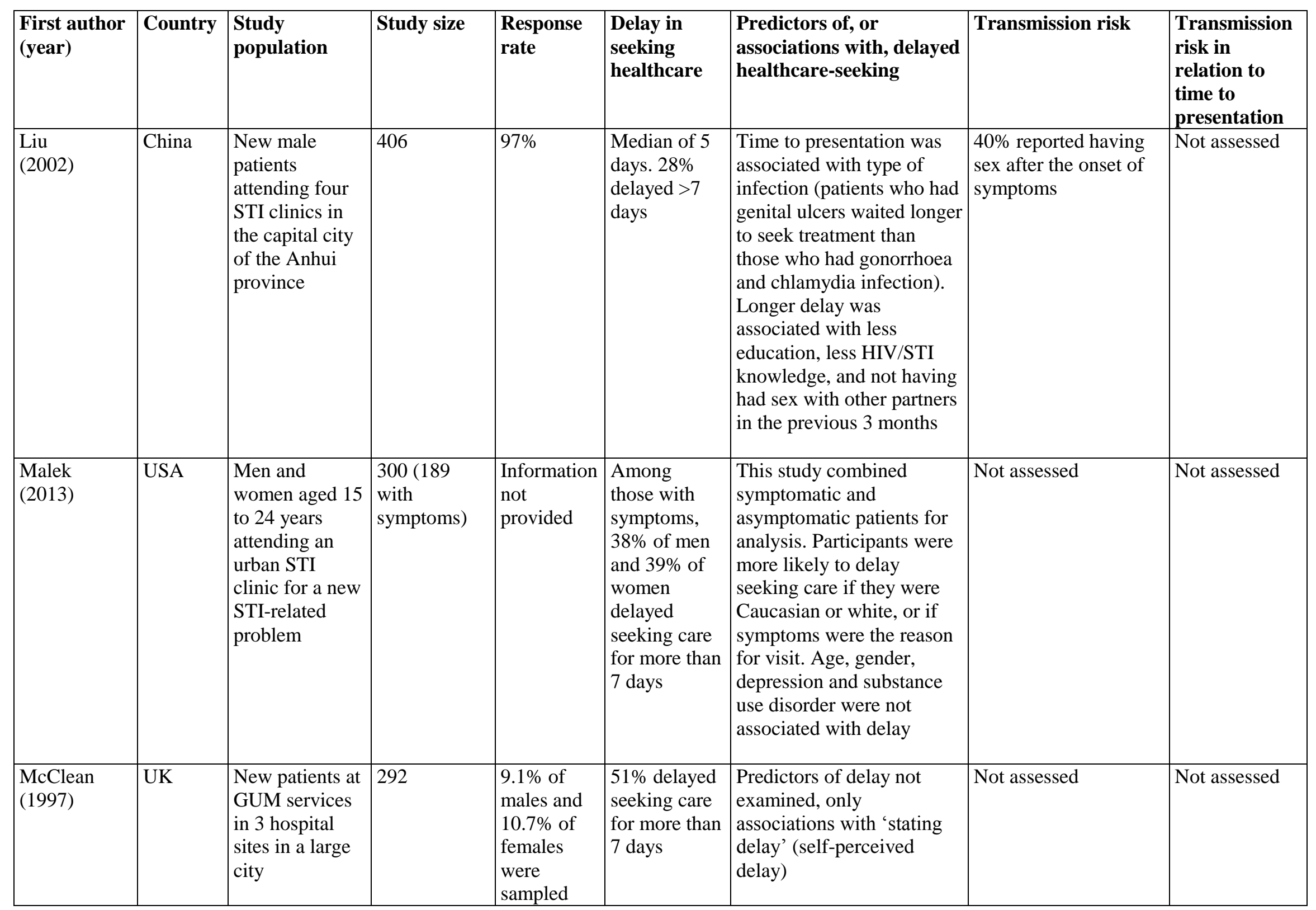




\begin{tabular}{|c|c|c|c|c|c|c|c|c|}
\hline $\begin{array}{l}\text { First author } \\
\text { (year) }\end{array}$ & Country & \begin{tabular}{|l|} 
Study \\
population
\end{tabular} & Study size & $\begin{array}{l}\text { Response } \\
\text { rate }\end{array}$ & \begin{tabular}{|l|} 
Delay in \\
seeking \\
healthcare
\end{tabular} & \begin{tabular}{|l|} 
Predictors of, or \\
associations with, delayed \\
healthcare-seeking
\end{tabular} & Transmission risk & $\begin{array}{l}\text { Transmission } \\
\text { risk in } \\
\text { relation to } \\
\text { time to } \\
\text { presentation }\end{array}$ \\
\hline $\begin{array}{l}\text { Mercer } \\
(2012)^{\mathrm{a}}\end{array}$ & UK & $\begin{array}{l}\text { New patients } \\
\text { attending } 4 \\
\text { GUM clinics } \\
\text { across England }\end{array}$ & $\begin{array}{l}1504(626 \\
\text { with } \\
\text { symptoms })\end{array}$ & $\begin{array}{l}\text { Range } \\
24.9 \%- \\
76.1 \%\end{array}$ & $\begin{array}{l}\text { Median of } 3 \\
\text { days }\end{array}$ & Not assessed & $\begin{array}{l}25.2 \% \text { of men and } \\
38.3 \% \text { of women } \\
\text { continued to have sex } \\
\text { before attending the } \\
\text { GUM clinic } \\
\end{array}$ & Not assessed \\
\hline $\begin{array}{l}\text { Meyer-Weitz } \\
(2000)\end{array}$ & $\begin{array}{l}\text { South } \\
\text { Africa }\end{array}$ & $\begin{array}{l}\text { Men and } \\
\text { women } \\
\text { attending two } \\
\text { STI clinics in } \\
\text { Cape Town } \\
\text { who had } \\
\text { symptoms }\end{array}$ & 1482 & $\begin{array}{l}1505 / 1535 \\
(98 \%)\end{array}$ & $\begin{array}{l}41 \% \text { waited } \\
>7 \text { days to } \\
\text { seek health } \\
\text { care }\end{array}$ & \begin{tabular}{|l|} 
Female gender, lower level \\
of education, unemployed, \\
no STI history, self-treated, \\
having friends who delayed \\
seeking treatment, not \\
talking to partners about \\
their STI, misconceptions \\
regarding cause of STIs, \\
and perceiving STIs not to \\
be serious were associated \\
with delay. No relation \\
between age and delay
\end{tabular} & Not assessed & Not assessed \\
\hline
\end{tabular}




\begin{tabular}{|c|c|c|c|c|c|c|c|c|}
\hline $\begin{array}{l}\text { First author } \\
\text { (year) }\end{array}$ & Country & \begin{tabular}{|l|} 
Study \\
population
\end{tabular} & Study size & $\begin{array}{l}\text { Response } \\
\text { rate }\end{array}$ & \begin{tabular}{|l|} 
Delay in \\
seeking \\
healthcare
\end{tabular} & $\begin{array}{l}\text { Predictors of, or } \\
\text { associations with, delayed } \\
\text { healthcare-seeking }\end{array}$ & Transmission risk & $\begin{array}{l}\text { Transmission } \\
\text { risk in } \\
\text { relation to } \\
\text { time to } \\
\text { presentation }\end{array}$ \\
\hline $\begin{array}{l}\text { Sawyer } \\
(1993)\end{array}$ & USA & $\begin{array}{l}\text { New patients } \\
\text { attending a } \\
\text { men's clinic at } \\
\text { a large public } \\
\text { university } \\
\end{array}$ & 66 & $100 \%$ & $\begin{array}{l}69.7 \% \text { waited } \\
>7 \text { days to } \\
\text { seek health } \\
\text { care }\end{array}$ & Not assessed & Not assessed & Not assessed \\
\hline $\begin{array}{l}\text { Thi Thu } \\
\text { (2007) }\end{array}$ & Vietnam & $\begin{array}{l}\text { Women } \\
\text { attending a } \\
\text { clinic for an } \\
\text { STI check-up } \\
\text { who were } \\
\text { subsequently } \\
\text { diagnosed with } \\
\text { an STI }\end{array}$ & $\begin{array}{l}60 \text { (43 with } \\
\text { symptoms) }\end{array}$ & \begin{tabular}{|l|}
$60 / 106$ \\
$(56.6 \%)$
\end{tabular} & $\begin{array}{l}\text { Median of } 30 \\
\text { days. Mean } \\
\text { of } 54 \text { days. } \\
82 \% \text { waited } \\
>7 \text { days to } \\
\text { seek health } \\
\text { care }\end{array}$ & $\begin{array}{l}\text { Women with lower } \\
\text { education delayed longer, } \\
\text { and urban women sought } \\
\text { care earlier than women } \\
\text { from rural areas. Delay was } \\
\text { associated with STI } \\
\text { knowledge but not with } \\
\text { attitudes to STIs. There } \\
\text { was no association between } \\
\text { delay and age or income }\end{array}$ & $\begin{array}{l}\text { Of the } 35 \text { women who } \\
\text { delayed seeking care, } \\
28(80 \%) \text { reported } \\
\text { having sex while } \\
\text { experiencing } \\
\text { symptoms. Of the } 8 \\
\text { women who did not } \\
\text { delay, } 3(38 \%) \text { had sex } \\
\text { while experiencing } \\
\text { symptoms. }\end{array}$ & $\begin{array}{l}\text { Women who } \\
\text { delayed } \\
\text { seeking care } \\
\text { were more } \\
\text { likely to } \\
\text { engage in sex } \\
\text { while having } \\
\text { symptoms than } \\
\text { women who } \\
\text { did not delay }\end{array}$ \\
\hline
\end{tabular}




\begin{tabular}{|c|c|c|c|c|c|c|c|c|}
\hline $\begin{array}{l}\text { First author } \\
\text { (year) }\end{array}$ & Country & $\begin{array}{l}\text { Study } \\
\text { population }\end{array}$ & Study size & $\begin{array}{l}\text { Response } \\
\text { rate }\end{array}$ & $\begin{array}{l}\text { Delay in } \\
\text { seeking } \\
\text { healthcare }\end{array}$ & $\begin{array}{l}\text { Predictors of, or } \\
\text { associations with, delayed } \\
\text { healthcare-seeking }\end{array}$ & Transmission risk & $\begin{array}{l}\text { Transmission } \\
\text { risk in } \\
\text { relation to } \\
\text { time to } \\
\text { presentation }\end{array}$ \\
\hline $\begin{array}{l}\text { Voeten } \\
(2004)\end{array}$ & Kenya & \begin{tabular}{|l|} 
People aged 13 \\
to 39 from \\
randomly \\
sampled \\
households in 4 \\
areas of Nairobi
\end{tabular} & $\begin{array}{l}1929(307 \\
\text { with STI } \\
\text { symptoms })\end{array}$ & $\begin{array}{l}1929 / 2365 \\
(81.6 \%)\end{array}$ & $\begin{array}{l}(\mathrm{n}=212) \\
\text { Median of } 7 \\
\text { days for both } \\
\text { women and } \\
\text { men }\end{array}$ & $\begin{array}{l}\text { In women, older age was } \\
\text { associated with delay. In } \\
\text { men, those who presumed } \\
\text { that their complaint was } \\
\text { caused by sex attended } \\
\text { sooner }\end{array}$ & $\begin{array}{l}35 \% \text { of men and } 62 \% \\
\text { of women continued to } \\
\text { have sex while } \\
\text { symptomatic }\end{array}$ & Not assessed \\
\hline $\begin{array}{l}\text { Zachariah } \\
(2002)\end{array}$ & Malawi & $\begin{array}{l}\text { New patients } \\
\text { diagnosed with } \\
\text { an STI } \\
\text { presenting at } \\
\text { STI clinic with } \\
\text { symptoms }\end{array}$ & 498 & $\begin{array}{l}\text { No details } \\
\text { given }\end{array}$ & $\begin{array}{l}\text { Median of } 14 \\
\text { days for } \\
\text { males, } 11 \\
\text { days for } \\
\text { females }\end{array}$ & Not assessed & $\begin{array}{l}42 \% \text { of men and } 58 \% \\
\text { of women had sex } \\
\text { while symptomatic }\end{array}$ & Not assessed \\
\hline
\end{tabular}

This table does not include studies assessing time to presentation for human immunodeficiency virus testing or for pelvic inflammatory disease.

${ }^{a}$ This paper contrasts data previously presented in Mercer et al. 2007 with new data collected 5 years subsequently. Only the new data is included in this line of the table to avoid duplication of information. Another paper (Gerressu et al. 2012) carried out secondary analysis on this dataset but is not included here.

${ }^{\mathrm{b}}$ This paper contrasts GUM clinic attendees with people attending GP based Locally Enhanced Services for sexual health (LESSH). The GUM clinic attendees were included in paper by Mercer et al. 2013, so only the LESSH data is included in this line of the table to avoid duplication. $\mathrm{GP}=$ general practitioner 


\subsubsection{Factors involved in healthcare-seeking behaviour}

How people make STI-related healthcare-seeking decisions is profoundly important for the prevention of STI transmission and acquisition. Understanding what factors influence decision-making about seeking an STI test, either in a positive or negative direction, is key to developing interventions and services that encourage testing behaviour (Ward et al., 1997). It is important to acknowledge that the decision to test does not take place in a vacuum; social and cultural conditions will affect healthcare-seeking behaviour and these need to be taken into consideration when building a comprehensive understanding of the process.

Qualitative research methods are ideal for studying in detail the process of going for an STI test because they can probe deeply into the social influences on individuals' decisionmaking, explore the relationship between meanings and actions, and obtain data that is not constrained by researchers' prior assumptions or questionnaire length (Power, 2002, Charmaz, 2004).

One barrier to STI testing which has received substantial attention from social science researchers is stigma (Major and O'Brien, 2005, Newton and McCabe, 2005, Malta et al., 2007, Mulholland and Van Wersch, 2007, Scambler, 2009, Balfe et al., 2010c, Hood and Friedman, 2011). STIs are regarded as stigmatised infections because being diagnosed with an STI suggests a violation of social norms and values, specifically that the individual has chosen to engage in behaviour often viewed as immoral, such as unprotected sex, sex with multiple partners, or sex with disreputable partners (Nack, 2000, Lichtenstein, 2003).

Research has shown individuals who score higher on measures of STI-related stigma are less likely to have had an STI test (Fortenberry et al., 2002, Rusch et al., 2008, Cunningham et al., 2009, Morris et al., 2014). Interestingly, Mulholland and Van Wesch (2007) argue that stigma may actually encourage some people to test, because individuals want to 'get rid of' the perceived contamination on their character.

Other factors which may prevent STI testing include embarrassment, feelings of invulnerability, fear of the test itself, or the cost of testing (Barth et al., 2002, Mimiaga et al., 2007, Balfe et al., 2012, Jackson and Roberts, 2015). For example, Barth et al. (2002) reported that $61 \%$ of the college students interviewed in their study specifically mentioned embarrassment when asked about factors relevant to the decision to seek an STI test. 
Shoveller et al. (2010) found that many of the young males in their study had been told fearinvoking stories by their peers about the STI testing procedure, and this had affected their willingness to seek testing. With regards to cost, the implications of charging for an STI test were demonstrated quite clearly when a co-payment of between US $\$ 15.00$ and US\$65.00 (around NZ\$20 - NZ\$90) introduced in an STI clinic in Denver, USA resulted in fewer visits $(-28.5 \%)$ and fewer diagnoses of chlamydia $(-28.1 \%)$ and gonorrhoea $(-38.1 \%)$ (Rietmeijer et al., 2005).

While the barriers to STI testing are well researched, the facilitators have received much less attention (Yeung et al., 2015). Education to combat the lack of knowledge about STIs has been identified as a key facilitator for testing (Yeung et al., 2015). Also, offering urine testing or a self-swab over urethral or cervical swab may aid testing acceptance rates (Heritage and Jones, 2008, Shoveller et al., 2010). Incentives such as competitions or vouchers may also be effective (Currie et al., 2010), although there is some evidence that these may be more effective among males than females (Rose et al., 2008).

Other than studies relating specifically to HIV testing (Lachowsky et al., 2014, Dickson et al., 2015), only one study has previously investigated healthcare-seeking for chlamydia testing in New Zealand. The study by Rose et al. (2008) was conducted in 2006 and explored barriers to chlamydia testing and methods to encourage testing with young people and healthprofessionals during focus groups. The study found similar results to the international studies described above. Reasons put forward as to why someone may not seek chlamydia testing were: lack of knowledge; fear of testing and treatment procedures; cost; stigma; and denial of personal risk. Ideas of ways to encourage testing included normalising testing, using incentives and advertising, and providing better education. No studies about healthcareseeking behaviour for STI testing have been conducted since 2006, meaning there is a lack of information about contemporary facilitators and barriers to STI testing among young people in New Zealand. 


\subsubsection{STI Knowledge}

Seeking STI testing is usually the decision of an individual, meaning timely receipt of care requires at a very minimum, recognition of a need to seek care. This recognition relies on knowledge of potential infections, transmission routes, symptoms and the possibility of asymptomatic infection. The WHO states in its 2016-2021 Global Health Sector Strategy on STIs that education, including a focus on increasing awareness of STIs, is critically important for STI prevention (World Health Organization, 2016a). The strategy goes on to say that when community knowledge about STIs is strengthened, and stigmatisation and discrimination are reduced, the use of services related to STIs tends to improve.

There is a wealth of literature about STI knowledge, although this is mainly related to how it is associated with sexual-risk behaviours such as condom use (Kellock et al., 1999, Diclemente et al., 2002, Burazeri et al., 2004, Mmbaga et al., 2008, Greaves et al., 2009) and STI acquisition (Shipitsyna et al., 2013, Vivancos et al., 2013, Grulich et al., 2014), rather than healthcare-seeking behaviour. The impact of HPV knowledge on HPV vaccine uptake has also been a focus of research in recent years (Marlow et al., 2013, Coles et al., 2015, Patel et al., 2016). Studies about STI knowledge and sexual behaviour have taken the form of cross-sectional association studies and intervention studies, and have shown mixed results. For example, some cross-sectional studies have shown that a low level of STI knowledge is associated with risky sexual behaviour such as high numbers of sexual partners and infrequent condom use (Rock et al., 2003, Burazeri et al., 2004, Mmbaga et al., 2008) whereas others have not found an association (Greaves et al., 2009, Kong et al., 2010, Norbu et al., 2013, Hoehn et al., 2016). Further still, some cross-sectional studies have reported that higher STI knowledge is actually associated with increased risky sexual behaviour (Andersson-Ellstrom and Milsom, 2002, Simpson et al., 2015). Intervention studies aiming to reduce sexual risk behaviour through improving knowledge have also shown mixed results (Tyden et al., 1994, Tyden et al., 1998, McClean et al., 2000, Lim et al., 2012). A systematic review of such interventions concluded that although many interventions improved sexual health knowledge, very few led to a significant reduction in sexual risk behaviour (Lazarus et al., 2010). 
Less is known about the impact of STI knowledge on healthcare-seeking behaviour for STI testing, although this does not apply to the specific case of HIV knowledge and HIV testing which has been much more widely researched (Berg, 2013, Naidoo and Taylor, 2015). The majority of the research into the effect of STI knowledge on general STI testing has been qualitative, where in-depth interviews with individuals have uncovered that lack of knowledge is a factor related to delayed or non-existent healthcare-seeking for STI testing (Malta et al., 2007, Chaudhary et al., 2008, Balfe et al., 2012).

In New Zealand, assessment of STI-related knowledge has focused on HIV and AIDS (Fergusson et al., 1994, Duncan and Bergen, 1997, Lachowsky et al., 2014, Henrickson et al., 2015), with many of the studies taking place in the 1980s at the beginning of the global HIV epidemic (Buisman et al., 1988, Lungley, 1988, Kilgour et al., 1990, Chetwynd, 1991). More recent studies have included that by Lachowsky et al. (2014), in which young gay and bisexual men who knew that HIV cannot pass through an undamaged latex condom were more likely (OR 1.35, 95\% CI 1.12 - 1.62) to have had a HIV test in the past year than those who did not know or were unsure. More information about the impact of general STI knowledge on testing for STIs other than HIV is needed.

\subsection{Testing and treatment of STIs}

An overview of STI testing is given in this section, including descriptions of service providers in New Zealand, methods of sample collection and laboratory diagnostic techniques. This is to provide a background of what healthcare and laboratory services are available in New Zealand. To complete the patient journey, treatment options are also described. Lastly, the threat of antibiotic resistance among STIs is briefly highlighted.

\subsubsection{Testing}

\subsubsection{Service providers in New Zealand}

There are several services that provide STI testing in New Zealand, including sexual health clinics (SHCs), Family Planning Clinics (FPCs), the New Zealand AIDS Foundation (NZAF), student and youth health clinics, local Māori and Pacific healthcare providers and 
general practitioners (GP). In New Zealand, the majority of STIs are diagnosed and managed by GPs (ESR Annual Surveillance Report 2014).

GP visits are only partly subsidised by the government, meaning patients are often required to make a co-payment for services. Some GPs provide free sexual health consultations for people less than 19 years of age. SHCs are publically-funded and provide free tests and treatment for STIs. Many of the youth health clinics are free, and the services at FPCs are free to under 22 year olds. These types of free services tend to be located in cities and some larger rural towns, meaning access to free sexual health care is restricted in more rural parts of the country (Morgan, 2013). In conclusion, funding for free sexual health consultations is irregular and depends on DHB, individual Primary Health Organisation and practice decisions.

\subsubsection{Off- site testing}

Off-site testing refers to STI testing that takes place outside of a health clinic setting, for example in the public restrooms of nightclubs or via home-based testing. This approach may be especially useful for screening hard to reach populations who don't attend healthcare providers.

Home-kits can be requested by individuals from private companies in many developed countries, including New Zealand, although concern about the reliability of the tests performed by these for-profit companies has been questioned (Alexander, 2011). Some countries are working with private companies to incorporate home-based sampling as a strategy to enhance uptake of STI screening. For example, the NCSP in England provides funding for remote testing carried out by Preventx Limited via an online service called Freetest.me. Currently, over 60 of the primary care trusts in England contract Preventx Limited to dispatch at-home chlamydia test kits as part of the NCSP (Preventx. www.preventx.com/nhs-screening).

Off-site testing has generally been found to be feasible and acceptable (Gotz et al., 2005, Macleod et al., 2005, Kong et al., 2009, Llewellyn et al., 2009, Vaughan et al., 2010, van Rooijen et al., 2016). In evaluation of effectiveness, many studies have reported that homebased testing increased screening rates (Lippman et al., 2007, Graseck et al., 2011, Shih et 
al., 2011, Reagan et al., 2012, Odesanmi et al., 2013), although this finding was not universal (Macleod et al., 2005, Domeika et al., 2007, Sacks-Davis et al., 2010). Importantly, a recent review of relevant RCTs concluded that increases in the proportion of individuals tested as a result of home-based collection, compared with clinic-based collection, are offset by a lower proportion of positive results (Fajardo-Bernal et al., 2015).

In New Zealand, a small study assessed whether a peer-led method of distributing off-site testing kits would be effective (Rose et al., 2010). In total, 67 kits were distributed to young people attending primary care service to pass on to their peers, however only three were returned to the laboratory for testing. Other than this study of kit distribution, the effectiveness of home-based STI testing has not been assessed in New Zealand to date.

\subsubsection{Method of sample collection}

Traditionally, samples for STI testing were taken from the cervix or urethra for the application of diagnostic assays such as culture and enzyme immunoassay. These techniques required viable organisms, sterile equipment and fastidious transport conditions (Jensen et al., 1988, Hocking et al., 2013). The introduction of NAATs has made possible the use of non-invasive specimens for testing because their enhanced sensitivity and ability to detect non-viable organisms allows for less stringent collection and storage requirements. Noninvasive samples include first-void urine, tampons, and vulva, introital, and vaginal swabs (Fairley et al., 1992, Tabrizi et al., 1996, Chernesky, 2005).

In New Zealand, many services offer self-taken vaginal swabs for females or urine sampling for males as an alternative to a clinician-taken swab for asymptomatic STI screening. Selfswabs have been shown to be acceptable and preferred by women in a range of clinical and cultural settings internationally, including in New Zealand (Serlin et al., 2002, Chernesky, 2005, Hoebe et al., 2006, Rose et al., 2007). Similarly, first-void urine is preferred by males (Balfe et al., 2012). Self-taken vulvovaginal swabs or first-void urine have been shown to have equal or better sensitivity and specificity to clinician-taken endocervical or urethral swabs for N. gonorrhoeae and C. trachomatis (Cook et al., 2005, Schoeman et al., 2012, Stewart et al., 2012, Lawton and Bromhead, 2013). Self-swabs are also valuable for extragenital sampling (e.g. rectum or pharynx), as demonstrated in a SHC study in the UK in which the introduction of routine self-taken extra-genital swabs led to a large rise in detected 
extra-genital chlamydia and gonorrhoea infection among MSM and women (Nyatsanza et al., 2016).

\subsubsection{Timing}

The timing of testing is extremely important for both initial testing and retesting. Due to the latent period where the infecting organism is replicating (Figure 2.10), testing too early may lead to the assay giving a false negative. For $C$. trachomatis and $N$. gonorrhoeae, testing should occur two weeks after the last sexual contact or after a specific event of concern, to allow for the appropriate latent period to elapse before testing. That said, if a patient has symptoms or is unlikely to come back for testing, it is recommended that testing is done at the time of presentation (NZSHS, 2008, NZSHS, 2014).

Timing should also be considered when retesting an individual for an infection as a test of cure. Retesting should not occur too soon after treatment, as NAATs may produce a false positive result due to the amplification of non-viable organism nucleic acids. A prospective study of 59 treated cervical and/or rectal chlamydia infections found that $42 \%$ of samples tested positive three weeks after treatment (Dukers-Muijrers et al., 2012). As such, the current New Zealand guideline recommends waiting at least five weeks before retesting for C. trachomatis if a test of cure is required (NZSHS, 2008). Because N. gonorrhoeae is extracellular (Nassif et al., 1999), clearance of organism material occurs faster than for $C$. trachomatis, so follow-up testing can take place at two weeks post-completion of treatment (Hjelmevoll et al., 2012, NZSHS, 2014, Wind et al., 2016).

\subsubsection{Laboratory diagnostics}

C. trachomatis and $N$. gonorrhoeae - Over the past couple of decades, NAATs have generally replaced culture as the preferred method of diagnosis for these organisms in most laboratories. The main advantages of NAATs over culture is their sensitivity, which allows more accurate diagnosis and the use of self-collected specimens. In addition, many NAAT platforms offer combined testing for C. trachomatis and $N$. gonorrhoeae, meaning these two microorganisms can be tested for at the same time using one sample, and at a similar cost to a one-sample test. 
While the introduction of NAAT tests has revolutionised chlamydia and gonorrhoea testing, there are some disadvantages that also need to be taken into consideration. The first is that NAATs have lower specificity than culture for $N$. gonorrhoeae and can therefore result in false positives. NAAT testing for $N$. gonorrhoeae can result in false positives due to commensal Neisseria species on the body incorporating $N$. gonorrhoeae DNA that corresponds to the gene targets of the NAATs (Whiley et al., 2006). This is particularly an issue for extra-genital specimens, such as pharynx, rectum and other non-genital swabs, because of the high load of commensal Neisserias in these sites as normal flora (Walsh et al., 2011). Therefore, extra-genital site testing should be considered on the basis of individual behavioural risk and knowledge of sexual networks, and additional confirmatory testing is recommended (NZSHS, 2014).

False positives are more likely to occur when screening for an infection in a population where it is not often seen, due to the positive predictive value (PPV) (Hocking et al., 2013). The PPV is the proportion of people who test positive for an infection who actually have the infection. As the prevalence of an infection in the population decreases, the PPV of the test decreases and the proportion of false positive test results increases. The prevalence of gonorrhoea is much lower than the prevalence of chlamydia in most populations (see Table 2.2), meaning false positives are again, more likely for gonorrhoea than for chlamydia.

The other disadvantage of NAATs is the inability to offer phenotypic information on antibiotic susceptibility. The swab lysis buffers that are utilised for many commercial NAAT assays destroy the organism in order to gain access to the nucleic material of the cell, meaning concurrent culture cannot take place (NZSHS, 2014). If an individual presents with symptoms suggestive of gonorrhoea, or reports sexual contact with someone diagnosed with gonorrhoea, an additional swab should be taken to enable antimicrobial susceptibility testing by culture (NZSHS, 2015c).

Herpes simplex virus (HSV) - HSV is not usually tested for in the absence of symptoms, as false negative results are relatively common due to intermittent viral shedding, and there is no curative treatment (Krantz et al., 2004, Tronstein et al., 2011). If a patient presents with symptoms, a diagnosis is based on history and clinical findings. Viral swabs can be taken 
from visible lesions and analysed to confirm infection using either NAAT or culture. In the case of no symptoms, an antibody test can be performed on a blood sample if necessary.

Human papillomavirus (HPV) - As discussed in section 2.2.3, HPV infection can be symptomless, cause genital warts or in some cases, cause lower genital tract and oropharyngeal pre-cancers and cancers (Hutter and Decker, 2016). As most sexually active people will contract HPV at some point in their lives, and many infections do not result in any clinical disease, HPV testing is not part of routine diagnostic testing. Instead, clinicians look for clinical symptoms and sign of disease that may be the result of a HPV infection.

In the case of genital warts, diagnosis is made on clinical grounds by visual inspection. The use of HPV DNA testing for anogenital wart diagnosis is not recommended because test results do not confirm the diagnosis and do not assist with genital warts management (NZSHS, 2015b).

Currently in New Zealand, testing for high risk (oncogenic) HPV types (HrHPV) is conducted in the triage and management of patients with abnormal cervical smears as assessed by cervical cytology (NZSHS, 2015b). However, as a result of international research showing that HPV testing is at least as effective as cytology (Ronco et al., 2014, Wright et al., 2015), the National Cervical Screening Programme is planning to move to primary HPV screening from 2018, meaning HrHPV testing will be the first step in the screening pathway (Primary HPV Screening, 2016).

Human Immunodeficiency Virus (HIV) - HIV is diagnosed by testing for antibodies to the virus in an individual's blood. As it can take three to twelve weeks for the body's immune system to make enough antibodies to be detected by the test, it is important to wait for a sufficient period after possible HIV exposure before testing (Busch and Satten, 1997). A HIV antibody test is usually conducted first with an enzyme-linked immunosorbent assay (ELISA) and confirmed with a Western blot in the case of a positive result. Point-of-care versions of ELISA tests for HIV are available, called HIV Rapid tests. The tests use just a few drops of blood and have been reported to have upwards of $97.7 \%$ sensitivity (Lyamuya et al., 2009, Chaillet et al., 2010), although it is recommended that individual tests are used in combination algorithms to increase accuracy (Wright and Stringer, 2004). HIV Rapid Cards 
enable point-of-care testing because a result is available in around 20 minutes, making it attractive to individuals who want to know their HIV status quickly. This technology is currently used by the NZAF for testing.

After diagnosis has been confirmed using antibody tests, a CD4 T cell count is usually conducted to assess which stage of disease an individual is at. A CD4 T cell count fewer than 200 cells $/ \mathrm{mm}^{3}$ (normal range: 500 to 1,600 cells $/ \mathrm{mm}^{3}$ ) is one of the qualifications for a diagnosis of stage 3 infection (AIDS) (Selik et al., 2014).

\subsubsection{Treatment}

Chlamydia and gonorrhoea can both be treated with antibiotics. Usually a single (oral) dose of azithromycin $(1 \mathrm{~g})$ cures chlamydia, although doxycycline $(100 \mathrm{mg})$ is recommended twice daily for a week for rectal infection or highly symptomatic infections (NZSHS, 2015d). The current New Zealand guidelines recommend treating gonorrhoea with ceftriaxone intramuscular injection $(500 \mathrm{mg})$ plus azithromycin oral dose $(1 \mathrm{~g})$. An alternative treatment option is to prescribe ciprofloxacin $(500 \mathrm{mg})$ plus azithromycin $(1 \mathrm{~g})$ (NZSHS, 2015d).

Antiviral therapy of recurrent genital herpes may be suppressive or episodic, although many individuals prefer a suppressive therapy regimen. Standard treatment in New Zealand for initial either primary or non-primary HSV is either acyclovir or valacyclovir (NZSHS, 2015d).

There are currently no antiviral drugs that target low-risk HPV infection, although warts may be treated by cryotherapy, surgical removal, or with topical agents that attack the wart tissue (Hutter and Decker, 2016). HrHPV infections in the context of cervical cancer screening are managed according to National guidelines (Guidelines for Cervical Screening in New Zealand, 2008).

HIV is treated with a combination antiretroviral therapy (ART), which reduces the amount of virus in the body, preventing the progression of HIV to AIDS and reducing the possibility of passing on the infection (Pau and George, 2014). These need to be taken daily and HIVpositive individuals need to attend medical follow-up for the rest of their lives to monitor potential side effects and disease progression. There are several classes of antiretrovirals, including nucleoside reverse transcriptase inhibitors (NRTIs) and non-nucleoside reverse 
transcriptase inhibitors (NNRTIs) which restrict replication of the virus, CCR5 inhibitors which prevent the virus binding to and entering immune cells, integrase inhibitors which block the virus from integrating its DNA into the host cell genome, and protease inhibitors which disrupt viral assembly (Pau and George, 2014). These medications are used together in different combinations (depending on health history, immune response and patient preference) to create a multi-faceted assault on HIV.

\subsubsection{Antimicrobial resistance}

Antimicrobial resistance arises when a microorganism which causes infection, such as a bacteria, is no longer susceptible to an antibiotic or antimicrobial medicine to which it was previously sensitive. Infections caused by resistant microorganisms fail to respond to conventional treatment, resulting in prolonged illness and an increased risk of death (World Health Organization, 2011a).

Microbes are able to develop antimicrobial resistance due to their highly developed adaptation capabilities, powered by genetic plasticity and rapid replication (Salmond and Welch, 2008). Resistance develops because the high usage of antibiotics applies a selective pressure that favours the resistant variants of an organism, thereby increasing their frequency in a population (Martinez and Baquero, 2002).

Antimicrobial resistance can be difficult to diagnose, often requiring additional diagnostic tests. Once an infection has been identified to be resistant to a conventional treatment, it can usually be treated with an alternative antibiotic. However, the number of antibiotics available is limited, and multi-drug resistance is becoming a reality for several organisms. $N$. gonorrhoeae is one such organism. It is a highly adapted pathogen and can readily acquire novel chromosomal and plasmid-mediated antimicrobial resistance within and across antibiotic classes (NZSHS, 2014). Over time, N. gonorrhoeae has developed resistance to first-line antimicrobials such as penicillin, tetracycline, macrolides and fluroquinolones including ciprofloxacin. Worryingly, isolates with decreased susceptibility to cephalosporins have now been reported in several countries (Cole et al., 2014, Unemo and Shafer, 2014, Lee et al., 2015). After this antibiotic fails, there are no more treatment options. As a result, $N$. gonorrhoeae is one of three organisms classified by the CDC as 'urgent' antibiotic threats (Centers for Disease Control and Prevention, 2013a). 
As the emergence and proliferation of bacteria resistant to an antimicrobial chemotherapy is directly related to the amount of the antimicrobial compound in the organism's environment (Thomas et al., 2014), antibiotics should only be used when appropriate. This strategy of 'antimicrobial stewardship' is currently the main method of slowing the development of antimicrobial resistance, and advocates for waiting for the results of tests before prescribing antibiotic medicines. However, in the case of gonorrhoea, the public health importance placed on limiting the spread of this infection means that antibiotics are still prescribed empirically if there is a high index of suspicion of infection.

A rapid point-of-care diagnostic test for diagnosing gonorrhoea and predicting resistance would mean that empirical prescribing could be avoided. In addition, antibiotics could be targeted appropriately based on susceptibility of the strain(s) causing their infection. This would potentially allow abandoned first-line treatments to be re-introduced for patients with $N$. gonorrhoeae infections susceptible to these drugs, aiding the reduction of selective pressure for resistance to cephalosporins, thus prolonging their effectiveness (Turner et al., 2014). Rapid point-of-care diagnostic tests would also increase the proportion of patients treated appropriately on the same day as the test, reducing infectious duration and thus onwards transmission. However, such a diagnostic tool unfortunately does not currently exist.

\subsection{Summary}

STIs are common infections, especially among young people and specific sub-populations. New Zealand has a particularly high chlamydia incidence compared to other countries, which cannot be entirely explained by national testing rates. Given the potential detrimental effects on physical and psychological health and wellbeing, along with the incurability of some and antibiotic resistance of others, STIs are a very serious public health issue.

To be successful in reducing the incidence of STIs, both primary prevention methods (condoms) and secondary prevention methods (test and treat) will need to be employed. National screening programmes have only been projected to be effective for chlamydia, and 
trials have proved ineffective in reducing incidence rates. Therefore, a better approach to identify and treat infection is to encourage those at risk of STIs to seek testing themselves. Reducing the duration of infection by identifying undiagnosed infection and treating or managing it appropriately has the potential to limit spread, as indicated by the epidemiological modelling equation of the basic reproductive number. As prompt treatment is key, it is important to know how long people wait to seek testing, and what are the barriers and motivators to testing. In addition, investigating alternative pathways to testing is valuable. This thesis will explore these issues, providing much needed knowledge about healthcare-seeking behaviour for STI testing in the New Zealand context. 


\section{Chapter 3 Healthcare-seeking behaviour of people with sexually transmitted infection symptoms attending an urban Sexual Health Clinic}

\subsection{Chapter Overview}

Before assessing the factors that may influence healthcare-seeking behaviour, we first need to understand the situation in New Zealand. This chapter describes the reasons people present for STI testing, assesses the length of time that those with symptoms wait before seeking healthcare, analyses the transmission risk in this period, and investigates factors associated with delayed healthcare-seeking behaviour among SHC attendees.

\subsection{Abstract}

Background: Untreated STIs may be transmitted to previously uninfected individuals, increasing the amount of infection in the population. Therefore, the early detection and subsequent management of STIs is crucial to control efforts. Time to presentation for STI symptoms and risk of transmission in this period has not been assessed in New Zealand to date.

Methods: All new clients presenting to an urban SHC were invited to complete a questionnaire which included demographic information, sexual health history and details about the clinic visit.

Results: Of 331 people approached, 243 (73.4\%) agreed to complete the questionnaire. Four incomplete questionnaires were excluded, leaving 239 participants. The most common reason for seeking healthcare was experiencing symptoms (39.4\%) and almost half (41.7\%) of people with symptoms waited $>7$ days to seek healthcare. There were no statistically significant predictors of delayed healthcare-seeking, potentially due to the small sample size in this analysis. Around a third $(30.6 \%)$ of people with symptoms had sex after they first thought they may need to seek healthcare. Infrequent condom use was reported more by people who had sex with existing partners than by people who had sex with new partners.

Conclusions: This is the first study to quantify healthcare-seeking behaviour for STI in New Zealand. Similar to studies from other developed countries, delayed healthcare-seeking was common and many people reported engaging in sex while symptomatic. Public health campaigns should emphasize prompt action for STI symptoms and the need to abstain from sex with all partners until healthcare has been sought and appropriate treatment and/or advice given. 


\subsection{Introduction}

STIs are a major public health problem. Untreated STIs can lead to serious health complications including PID, infertility and adverse pregnancy outcomes, and also increased susceptibility to further STI acquisition including HIV (Idahl et al., 2004, Haggerty et al., 2010, Ward and Ronn, 2010). In addition, those infected may pass on the organism to previously uninfected individuals, increasing the amount of infection in the population. Therefore, the early detection and subsequent management of infection is a central issue in the control of STIs.

International studies have shown that $20 \%$ to $60 \%$ of adults wait longer than seven days before seeking care for STI symptoms (Leenaars, 1993, Akinnawo and Oguntimehin, 1997, Fortenberry, 1997, Hook et al., 1997, Khan et al., 2005). Studies have also reported that many people continue to have sex after noticing symptoms (Moses et al., 1994, Fortenberry, 1997, Irwin et al., 1999, Voeten et al., 2004), potentially transmitting the infection to others. While it is assumed that delayed healthcare-seeking is associated with a higher likelihood of sex while symptomatic, few studies have directly assessed the association.

To the author's knowledge, the time to presentation for STI symptoms has not been quantified in New Zealand other than for those with genital warts (Ireland et al., 2005), and there has been no assessment of sexual behaviour while symptomatic. It is therefore not known whether delayed healthcare-seeking for STI symptoms is common in this population, or whether it has a potential role in STI transmission. This is important because incidence rates of common STIs such as chlamydia and gonorrhoea are high in New Zealand (ESR Annual Surveillance Report 2013).

The objectives of this study were to determine the most common reasons for seeking an STI test, quantify time to presentation for STI testing in people with STI symptoms, determine the factors associated with delayed healthcare-seeking, assess transmission risk, and to identify the predictors of positive diagnosis. 


\subsection{Methods}

\subsubsection{Study design}

A cross-sectional single-centre observational study design was used to investigate healthcareseeking behaviour and associated factors.

\subsubsection{Setting and sample}

The study was conducted at an inner-city public SHC in Wellington, New Zealand. The clinic's services include STI testing, treatment and advice, emergency contraception and sexual assault care, as well as accepting referrals from primary and secondary level clinicians for complex sexual health problems. Anyone may attend for acute STI management free of charge.

Only new clients to the clinic were sampled to avoid including people who were attending for follow-up treatment or for a test of cure, as the study outcome measures were related to healthcare-seeking behaviour for new symptoms. Other than restricting to existing clients, there were no specific exclusion criteria.

\subsubsection{Recruitment}

All new clients attending the SHC between September and November 2015 were provided with a study pack by the reception staff before their consultation which included a Participant Information Sheet and a copy of the questionnaire. Those that agreed to take part in the study filled out the questionnaire in the waiting room area and returned the completed copy to reception staff who added their clinic patient number. Participants were also given the option to complete the questionnaire online using secure encrypted surveying software, although only one participant participated via this route. Any refusals to take part were recorded by the reception staff on the top of the questionnaire and filed separately for collection by the researcher. Questionnaires were anonymous, apart from the clinic patient number that was used to link the answers from the questionnaire to STI diagnosis/es.

\subsubsection{Measures}

The questionnaire was developed from existing literature on this subject; especially influential were the linked 'Patient Access and the Transmission of Sexually-transmitted 
Infections' (PATSI) and 'Maximising STI Control' (MSTIC) studies in the UK (Mercer et al., 2007, Aicken et al., 2011a). The draft questionnaire was refined in consultation with clinic staff. Cognitive testing was conducted with a group of young people to ensure that there were no questionnaire items that could be misunderstood or were too difficult to answer, which would likely result in missing or incorrect data. The questionnaire included items on basic demographic information, sexual health history including previous STI testing and previous diagnosis, and details about the patient's visit to the clinic (see Appendix A). The primary outcome measures for this study were reason for testing, number of days between symptom onset and contacting health services, sexual behaviour of symptomatic respondents (including partner status and condom use), and STI diagnosis/es.

Participants could select from a list of ten possible answers their reason for testing, or write in their own reason; these answers were then collapsed into seven categories for analysis. Multiple answers were allowed and in this case a prioritisation approach was employed to allocate individuals to a single group following the hierarchy of: symptoms >partner symptoms $>$ had unprotected sex $>$ intend to have unprotected sex $>$ referred or called in $>$ just a check-up>other.

Delay was defined as waiting more than seven days to contact health services after the onset of symptoms. This is the most common definition of delay found in the literature (Leenaars, 1993, Moses et al., 1994, Irwin et al., 1999, Khan et al., 2005, Mercer et al., 2007, Malek et al., 2013).

Those that indicated they were or had been experiencing symptoms were asked how many days the symptoms were present before they contacted any health services, if they had visited another health provider before coming to the clinic, and if they had attempted any selftreatment in this time. They were also asked whether they'd had sex since first thinking they may need to go to a clinic or health services, and with how many total partners and new partners. Lastly, they were asked to indicate how often they had used condoms in this period using a five-point scale ranging from 'none of the time' to 'all of the time'. This scale was then categorised into two groups; frequent condom use ('all of the time', 'more than half of the time') and infrequent condom use ('half of the time', 'some of the time', 'none of the time'). 
Māori and Pacific peoples are disproportionately affected by STIs according to laboratorybased surveillance data in New Zealand (ESR Annual Surveillance Report 2014), therefore ethnicity was analysed as a predictor variable for some of the analyses in this chapter. If an individual identified with multiple ethnicities, a prioritisation approach was used to allocate individuals to a single group using the hierarchy Māori>Pacific peoples>Asian>other groups except New Zealand European>New Zealand European), as has been previously used in New Zealand Ministry of Health publications (Cormack, 2010).

\subsubsection{Data linkage}

The questionnaire included an item which asked for consent to access the participants' STI results for use in the study. When consent was given, a member of the clinical staff accessed the patient records and recorded any positive diagnosis/es. Infections were restricted to bacterial or viral STIs and the protozoal infection trichomoniasis. Candidiasis and bacterial vaginosis $(\mathrm{BV})$ were excluded as they may not have been sexually transmitted.

\subsubsection{Statistical analysis}

Pearson's chi-square and Fisher's exact test were used to compare demographic characteristics and to determine the association with reason for testing. Logistic regression was used to identify factors associated with healthcare-seeking delay ( $>7$ days). Predictors included gender, age, ethnicity, education, sexual risk (defined as two or more partners in the past year and infrequent condom use), sexual orientation behaviour (deduced from answers to number of male and female partners in the past year), previously tested, previously diagnosed with an STI, first visit for this set of symptoms, and self-treating. Multiple logistic regression analyses were also performed to estimate adjusted odds ratios (adjusting for gender, age and ethnicity).

Where data was missing for an item, the participant was excluded from the particular analysis. Two-tailed tests were used and $p$ values $<0.05$ were considered statistically significant. The analyses were conducted with Statistical Package for the Social Sciences (SPSS) Statistics version 22. 


\subsubsection{Ethical approval}

Ethical approval was granted by Victoria University of Wellington Human Ethics Committee (ref: 20504) (Appendix D).

\subsection{Results}

Of 331 new patients who were approached to take part, 243 (73.4\%) agreed. Four respondents completed less than 50\% of the questionnaire and so were excluded from analysis, leaving 239 responses in the final dataset. Questionnaire coverage was good with over $98 \%$ of respondents completing all of the demographic and sexual history variables of interest, with the exception of number of sexual partners in the past year which was missed by $7.5 \%$ of respondents. Of the 91 people who attended the SHC due to experiencing symptoms, $92 \%$ answered all of the questions relating to contacting healthcare and $88.5 \%$ answered the relevant questions on sexual activity while symptomatic.

The sample was split fairly evenly between males and females, with almost half under the age of 25 (Table 3.1). The participants were very well educated with almost two thirds reporting a degree as their highest qualification. 
Table 3.1. Demographic characteristics

\begin{tabular}{lcc}
\hline & Denominator* & $\boldsymbol{n}(\boldsymbol{\%})$ \\
\hline Gender: & 239 & \\
Female & & $113(47.3)$ \\
Male & & $126(52.7)$ \\
Age (yrs): & 235 & \\
$\quad<25$ & & $103(43.8)$ \\
$25-34$ & & $94(40.0)$ \\
$35+$ & 236 & $38(16.2)$ \\
Ethnicity: & & $110(46.6)$ \\
New Zealand European & & $16(6.8)$ \\
Māori & & $3(1.3)$ \\
Pacific peoples & & $13(5.5)$ \\
Asian & & $94(39.8)$ \\
Other & 239 & \\
Education: & & $95(39.7)$ \\
High School or less & & $144(60.3)$ \\
Tertiary qualification & &
\end{tabular}

The clinic provided anonymised routinely collected data on all participants who attended the clinic during the study period which was used to compare the gender, age, and ethnicity of those who completed the questionnaire with the general clinic population. Pearson's chisquared tests revealed no significant differences between the study sample and the whole clinic sample for gender or ethnicity, however there was a statistically significant difference in age between the two groups $\left(X^{2}(2)=40.58, p<0.001\right)$ where the study sample was younger than the overall clinic sample $(43.8 \%<25 \mathrm{yrs}$ vs $26 \%<25 \mathrm{yrs})$.

The most common reason people attended the clinic for an STI test was because they had developed genital symptoms (Table 3.2). Other common reasons included a partner having symptoms or testing positive, having had unprotected sex with a new partner, or just wanting a check-up. To determine whether reason for testing was related to sociodemographic characteristics (gender, age and education level), several Fisher's exact tests were carried out. Differences in reason for testing were not examined by ethnicity due to the small numbers in some of the ethnic categories. Reason for testing did not differ by gender $(p=0.148)$, but 
there was a statistically significant association between age and reason for testing $(p=$ 0.032). Further exploration showed that this was driven by an association between age and testing due to intending to have unprotected sex $(p=0.001)$, where $3.0 \%$ of under $25 \mathrm{~s}$ were getting tested because they intended to have unprotected sex with a new partner, compared to $7.8 \%$ of those $25-34$ years and $23.7 \%$ of those over 35 years old. Overall, there was no association between education and reason for testing $(p=0.088)$, although individual analyses indicated that those who had a tertiary qualification were more likely to select 'just a check-up' as their reason for testing than those whose highest qualification was high school or less $(p=0.026)$.

Table 3.2. Reasons for attending for current test, previous testing behaviour and previous diagnoses

\begin{tabular}{lcc}
\hline Testing behaviour & Total N* & $\boldsymbol{n}(\boldsymbol{\%})$ \\
\hline Reason for seeking an STI test & 231 & \\
Developed genital symptoms & & $91(39.4)$ \\
Partner has symptoms or tested positive & $30(13.0)$ \\
Had unprotected sex & $41(17.7)$ \\
Intends to have unprotected sex & $19(8.2)$ \\
No symptoms, just a check-up & $29(12.6)$ \\
Being referred or called in & $5(2.2)$ \\
Other & 234 & $16(6.9)$ \\
Previous testing behaviour & & $146(62.4)$ \\
Had an STI test before & & $88(37.6)$ \\
Never had an STI test before & & \\
Previously diagnosed with an STI (of those who had tested before) & 142 & \\
Yes & & $88(40.8)$ \\
No & & $84.2)$ \\
\hline
\end{tabular}

${ }^{*}$ Number of participants that answered this question

Many people had been tested before (62.4\%) and females were more likely to have had a previous STI test than males after adjustment for age (57.1\% of males, $65.5 \%$ of females, OR $=1.906, p=0.026$ ). Of those who had been tested, around $40 \%$ had been diagnosed with an 
STI (Table 3.2), this corresponds to $24.3 \%$ of the sample having been previously diagnosed with an STI (24.6\% of men, $23.9 \%$ of women, no significant difference). The most common STI that people reported being previously diagnosed with was chlamydia (64.9\% of people who answered this question).

For those with symptoms ( $n=91)$, the median length of time between symptom onset and contacting health services was 5.5 days (range $0-750$ days). The distribution of delay times is shown in Figure 3.1. Almost half (41.7\%) of respondents waited more than seven days from symptom onset to contacting health services (Table 3.3).

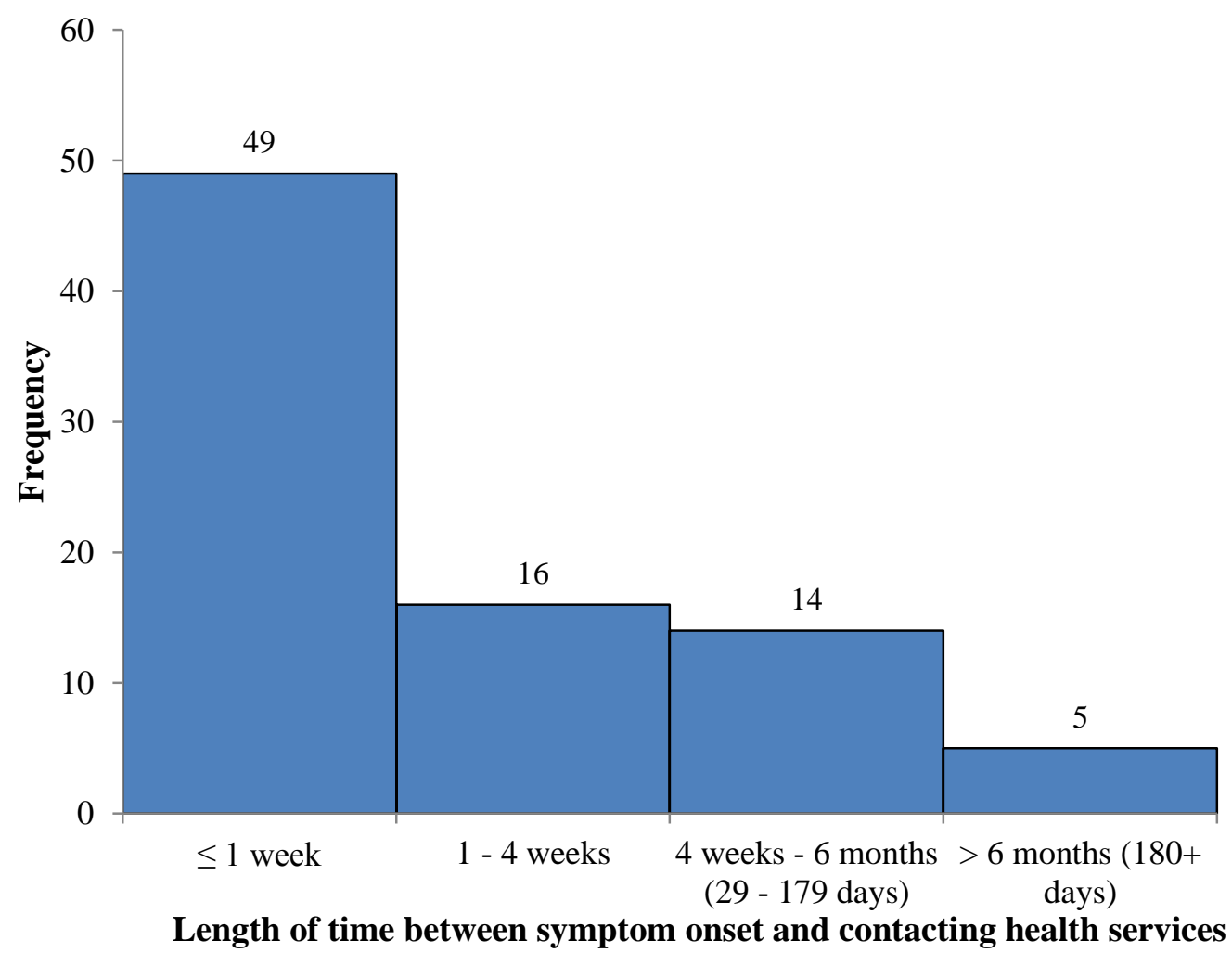

Figure 3.1. Histogram showing the distribution of delay behaviour 
Table 3.3. Healthcare-seeking and sexual behaviour of symptomatic respondents $(n=$ 91)

\begin{tabular}{lcc}
\hline Behaviour & Denominator $^{*}$ & $\boldsymbol{n}(\boldsymbol{\%})$ \\
\hline Previously visited a health provider for this set of symptoms & 87 & $22(25.3)$ \\
Attempted self-treatment & 85 & $22(25.9)$ \\
Waited longer than 7 days to visit a health provider & 84 & $35(41.7)$ \\
& & Median (IQR) \\
Days between symptom onset and contacting health services & 84 & $5.5(3.0-18.0)$ \\
\hline
\end{tabular}

*Number of participants who answered this question

Logistic regression was used to obtain crude and adjusted (for gender, age, ethnicity) odds ratios, which revealed no statistically significant associations with delay for gender, age, ethnicity, education, sexual risk, sexual orientation behaviour, previous testing, previous diagnosis, first visit $(\mathrm{y} / \mathrm{n})$, self-treatment $(\mathrm{y} / \mathrm{n})$, or type of STI (lesion type or non-lesion type) (all $p>0.05$ ) (Table 3.4). However, the sample size for this particular analysis was small and so it is likely that it was underpowered to detect any associations. 
Table 3.4. Predictors of delayed healthcare-seeking ( 7 days)

\begin{tabular}{|c|c|c|c|c|c|c|c|c|c|c|}
\hline & $n$ & $\begin{array}{c}\text { Median time to seek } \\
\text { help, days (mean) }\end{array}$ & $\begin{array}{c}\% \text { waiting }>7 \\
\text { days to seek help }\end{array}$ & OR & $95 \% \mathrm{CI}$ & $p$ & $n$ & $\begin{array}{c}\text { Adjusted } \\
\text { OR* }\end{array}$ & $95 \% \mathrm{CI}$ & $p$ \\
\hline Gender & 84 & & & & & & & & & \\
\hline Male & 46 & $5.0(41.7)$ & 39.1 & 1.000 & & & & - & & \\
\hline Female & 38 & $6.5(24.1)$ & 44.7 & 1.259 & $0.527-3.010$ & 0.604 & & - & - & - \\
\hline Age & 82 & & & & & & & & & \\
\hline$<25$ & 38 & $5.0(25.3)$ & 36.8 & 1.000 & & & & - & & \\
\hline $25-34$ & 31 & $8.5(46.0)$ & 51.6 & 1.829 & $0.697-4.798$ & 0.220 & & - & - & - \\
\hline $35+$ & 13 & $5.0(31.7)$ & 30.8 & 0.762 & $0.198-2.938$ & 0.693 & & - & - & - \\
\hline Ethnicity & 82 & & & & & & & & & \\
\hline New Zealand European & 46 & $6.50(41.24)$ & 41.3 & 1.000 & & & & - & & \\
\hline Māori & 10 & $6.75(27.65)$ & 50.0 & 1.421 & $0.361-5.601$ & 0.616 & & & & \\
\hline Asian & 5 & $7.00(41.00)$ & 40.0 & 0.947 & $0.144-6.227$ & 0.955 & & & & \\
\hline Other & 21 & $5.00(21.31)$ & 38.1 & 0.874 & $0.303-2.520$ & 0.804 & & - & - & - \\
\hline Education & 84 & & & & & & 80 & & & \\
\hline High school or less & 41 & $5.0(25.2)$ & 39.0 & 1.000 & & & 39 & 1.000 & & \\
\hline Tertiary educated & 43 & $7.0(41.9)$ & 44.2 & 1.237 & $0.519-2.951$ & 0.632 & 41 & 2.021 & $0.647-6.314$ & 0.226 \\
\hline Sexual risk ${ }^{\mathrm{a}}$ & 78 & & & & & & 75 & & & \\
\hline No risky sexual behaviour & 54 & $7.0(43.9)$ & 48.1 & 1.000 & & & 53 & 1.000 & & \\
\hline Risky sexual behaviour & 24 & $5.0(18.0)$ & 33.3 & 0.538 & $0.198-1.468$ & 0.226 & 22 & 0.577 & $0.186-1.793$ & 0.342 \\
\hline Sexual orientation behaviour ${ }^{\mathrm{b}}$ & 80 & & & & & & 76 & & & \\
\hline Non-MSM & 27 & $5.0(19.9)$ & 40.7 & 1.000 & & & 26 & 1.000 & & \\
\hline MSM & 16 & $7.0(85.8)$ & 43.8 & 1.131 & $0.324-3.952$ & 0.847 & 15 & 1.336 & $0.342-5.219$ & 0.677 \\
\hline Women ${ }^{\mathrm{c}}$ & 37 & $6.0(24.3)$ & 43.2 & 1.108 & $0.405-3.031$ & 0.841 & 35 & 0.906 & $0.296-2.773$ & 0.862 \\
\hline Previously tested & 81 & & & & & & 77 & & & \\
\hline Never had an STI test & 33 & $7.0(38.2)$ & 48.5 & 1.000 & & & 32 & 1.000 & & \\
\hline Tested previously & 48 & $4.5(30.0)$ & 33.3 & 0.531 & $0.214-1.318$ & 0.173 & 45 & 0.537 & $0.201-1.432$ & 0.214 \\
\hline Previously diagnosed (if tested) & 48 & & & & & & 45 & & & \\
\hline Never had an STI & 28 & $4.5(11.5)$ & 28.6 & 1.000 & & & 26 & 1.000 & & \\
\hline Previously diagnosed with an STI & 20 & $4.5(55.9)$ & 40.0 & 1.667 & $0.495-5.609$ & 0.409 & 19 & 1.415 & $0.277-7.230$ & 0.676 \\
\hline
\end{tabular}




\begin{tabular}{|c|c|c|c|c|c|c|c|c|c|c|}
\hline & $n$ & $\begin{array}{c}\text { Median time to seek } \\
\text { help, days (mean) }\end{array}$ & $\begin{array}{c}\% \text { waiting }>7 \\
\text { days to seek help }\end{array}$ & OR & $95 \% \mathrm{CI}$ & $p$ & $n$ & $\begin{array}{c}\text { Adjusted } \\
\text { OR* }\end{array}$ & $95 \% \mathrm{CI}$ & $p$ \\
\hline First visit & 83 & & & & & & 80 & & & \\
\hline Not first visit for this set of symptoms & 21 & $5.0(60.5)$ & 42.9 & 1.000 & & & 21 & 1.000 & & \\
\hline First visit for this set of symptoms & 62 & $7.0(25.2)$ & 41.9 & 0.963 & $0.354-2.619$ & 0.941 & 59 & 1.002 & $0.341-2.939$ & 0.997 \\
\hline Self-treatment & 82 & & & & & & 78 & & & \\
\hline Not attempted self-treatment & 61 & $5.0(34.3)$ & 37.7 & 1.000 & & & 59 & 1.000 & & \\
\hline Attempted self-treatment & 21 & $14.0(35.4)$ & 57.1 & 2.203 & $0.804-6.033$ & 0.124 & 19 & 2.271 & $0.726-7.100$ & 0.159 \\
\hline Infection diagnosis at current visit & 22 & & & & & & 22 & & & \\
\hline Non-lesion type infection ${ }^{\mathrm{d}}$ & 13 & $4.0(19.23)$ & 30.8 & 1.000 & & & 13 & & & \\
\hline Lesion type infection ${ }^{\mathrm{e}}$ & 9 & $5.0(53.56)$ & 44.4 & 1.800 & $0.308-10.517$ & 0.514 & 9 & 7.746 & $\begin{array}{l}0.373- \\
160.976\end{array}$ & 0.186 \\
\hline
\end{tabular}

\section{a As defined by 2 or more sexual partners in the past year and infrequent condom use}

${ }^{\mathrm{b}} \mathrm{OR}$ adjusted for age and ethnicity only as these groups are split by gender

${ }^{c}$ This category comprises all women, as the number of women who had sex with women in the past year was too small to use as a separate category in this analysis

${ }^{\mathrm{d}}$ Chlamydia/gonorrhoea/non-specific urethritis

${ }^{\mathrm{e}}$ Genital herpes/human papillomavirus

MSM = men who have sex with men 
Of those with symptoms who answered the section on sexual behaviour $(n=85), 26(30.6 \%)$ had continued to have sex after symptom onset. Of those who reported sex while symptomatic, 13 had had sex with their existing partner and 10 people had had sex with at least one new partner ( 3 did not report partner status). Infrequent condom use was reported more commonly by those who had sex with existing partners $(84.6 \%)$ than by those who had sex with new partners $(10.0 \%)$ (Table 3.5). Those who waited more than seven days to contact health services were more likely to have sex with any partner (existing or new) than those who waited less than seven days, $\mathrm{OR}=3.25,95 \% \mathrm{CI} 1.225-8.623, p=0.018$.

Table 3.5. Sexual behaviour since symptom onset and contacting healthcare services

\begin{tabular}{lccc}
\hline Sexual behaviour & & Infrequent condom use \\
& Total $\mathbf{N}$ & $\boldsymbol{n}(\boldsymbol{\%})$ & $\boldsymbol{n}(\boldsymbol{\%})$ \\
\hline Sex while symptomatic & 85 & $26(30.6)$ & $12(46.1)$ \\
Sex while symptomatic with existing partner only & 82 & $13(15.9)$ & $11(84.6)$ \\
Sex while symptomatic with at least one new partner & 82 & $10(12.2)$ & $1(10.0)$ \\
\hline
\end{tabular}

In total, 145 (61.4\%) participants gave permission for their STI test results to be extracted from the clinic records for use in the study. There were no demographic differences between participants who consented to linkage and those that did not. Of those that were symptomatic, $63(69.2 \%)$ gave permission, and $25(39.7 \%)$ of these people were positive. Among symptomatic participants with a positive STI result, $32 \%$ were caused by $C$. trachomatis, $24 \%$ by HPV (genital warts), $16 \%$ by HSV and $16 \%$ were cases of non-specific urethritis. Among asymptomatic patients who were diagnosed with an STI $(n=8)$, the majority $(75 \%)$ were due to $C$. trachomatis.

Of the 146 participants with linked STI results, 33 (22.6\%) had a positive test result. Of those with a positive test result, $24.2 \%(\mathrm{n}=8)$ were asymptomatic. Logistic regression was used to obtain crude odds ratios, which indicated that those who had had an STI test before were less likely to be diagnosed with an STI at this visit $(\mathrm{OR}=0.429,95 \% \mathrm{CI} 0.193-0.956, p=$ 0.038). The results of this analysis also indicated that those who had symptoms were more likely to be diagnosed with an STI $(\mathrm{OR}=6.086,95 \%$ CI $2.506-14.775, p<0.001)$ and 
those who had attempted self-treatment were less likely to be diagnosed with an STI at this visit $(\mathrm{OR}=0.236,95 \%$ CI $0.059-0.943, p=0.041)$ (Table 3.6). After adjustment for gender, age and ethnicity, men who had sex with men were more likely to receive a positive STI diagnosis compared to non-MSM (OR $=3.451,95 \%$ CI $1.071-11.121, p=0.038)$.

Being symptomatic remained a significant predictor of positive STI diagnosis after adjusting for gender, age and ethnicity $(\mathrm{OR}=8.372,95 \%$ CI $3.000-23.360, p<0.001)$, but both previous testing and attempting self-treatment became non-significant after adjustment $(\mathrm{OR}=$ $0.512,95 \%$ CI $0.215-1.223, p=0.132$ and $\mathrm{OR}=0.265,95 \%$ CI $0.057-1.237, p=0.091$ respectively) (Table 3.6). Those who had previously been diagnosed with an STI were more likely to be diagnosed with an STI at this visit, but the association was not statistically significant before or after adjustment $(\mathrm{OR}=2.769,95 \%$ CI $0.888-8.641, p=0.079$ and OR $=4.189,95 \%$ CI $0.953-18.417, p=0.058$ respectively). 
Table 3.6. Predictors of positive diagnosis at current visit

\begin{tabular}{|c|c|c|c|c|c|c|c|c|}
\hline 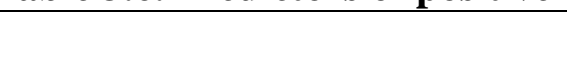 & $n$ & OR & $95 \% \mathrm{CI}$ & $p$ & $n$ & Adjusted OR* & $95 \% \mathrm{CI}$ & $p$ \\
\hline Gender & 146 & & & & & & & \\
\hline Male & 78 & 1.000 & & & & - & & \\
\hline Female & 68 & 0.491 & $0.218-1.107$ & 0.086 & & - & - & - \\
\hline Age & 143 & & & & & & & \\
\hline$<25$ & 64 & 1.000 & & & & - & & \\
\hline $25-34$ & 54 & 0.682 & $0.280-1.660$ & 0.399 & & - & - & - \\
\hline $35+$ & 25 & 0.947 & $0.322-2.785$ & 0.922 & & - & - & - \\
\hline Ethnicity & 145 & & & & & & & \\
\hline New Zealand European & 71 & 1.000 & & & & - & & \\
\hline Māori & 11 & 2.647 & $0.717-9.771$ & 0.144 & & - & - & - \\
\hline Pacific peoples & 2 & 3.176 & $0.188-53.553$ & 0.423 & & & & \\
\hline Asian & 6 & 3.176 & $0.586-17.225$ & 0.180 & & & & \\
\hline Other & 55 & 0.463 & $0.177-1.213$ & 0.117 & & & & \\
\hline Education & 146 & & & & 142 & & & \\
\hline High school or less & 53 & 1.000 & & & 51 & 1.000 & & \\
\hline Tertiary educated & 93 & 0.517 & $0.235-1.137$ & 0.101 & 91 & 0.494 & $0.192-1.274$ & 0.145 \\
\hline Sexual risk ${ }^{\mathrm{a}}$ & 139 & & & & 135 & & & \\
\hline No risky sexual behaviour & 82 & 1.000 & & & 80 & 1.000 & & \\
\hline Risky sexual behaviour & 57 & 0.580 & $0.251-1.343$ & 0.204 & 55 & 0.550 & $0.213-1.420$ & 0.217 \\
\hline Sexual orientation behaviour ${ }^{\mathrm{b}}$ & 138 & & & & 134 & & & \\
\hline Non-MSM & 53 & 1.000 & & & 52 & 1.000 & & \\
\hline MSM & 20 & 2.795 & $0.939-8.321$ & 0.065 & 20 & 3.451 & $1.071-11.121$ & 0.038 \\
\hline Women ${ }^{\mathrm{c}}$ & 65 & 0.696 & $0.279-1.735$ & 0.437 & 62 & 0.752 & $0.266-2.122$ & 0.590 \\
\hline Previously tested & 142 & & & & 138 & & & \\
\hline Never had an STI test & 53 & 1.000 & & & 52 & 1.000 & & \\
\hline Tested previously & 89 & 0.429 & $0.193-0.956$ & 0.038 & 86 & 0.512 & $0.215-1.223$ & 0.132 \\
\hline Previously diagnosed (if tested) & 89 & & & & 86 & & & \\
\hline Never had an STI & 54 & 1.000 & & & 52 & 1.000 & & \\
\hline Previously diagnosed with an STI & 35 & 2.769 & $0.888-8.641$ & 0.079 & 34 & 4.189 & $0.953-18.417$ & 0.058 \\
\hline
\end{tabular}




\begin{tabular}{|c|c|c|c|c|c|c|c|c|}
\hline & $n$ & OR & $95 \% \mathrm{CI}$ & $p$ & $n$ & Adjusted OR* & $95 \% \mathrm{CI}$ & $p$ \\
\hline First visit & 61 & & & & 59 & & & \\
\hline Not first visit for this set of symptoms & 14 & 1.000 & & & 14 & 1.000 & & \\
\hline First visit for this set of symptoms & 47 & 1.221 & $0.354-4.216$ & 0.752 & 45 & 2.411 & $0.530-10.963$ & 0.255 \\
\hline Self-treatment & 59 & & & & 57 & & & \\
\hline Not attempted self-treatment & 42 & 1.000 & & & 42 & 1.000 & & \\
\hline Attempted self-treatment & 17 & 0.236 & $0.059-0.943$ & 0.041 & 15 & 0.265 & $0.057-1.237$ & 0.091 \\
\hline Symptomatic status & 145 & & & & 141 & & & \\
\hline Asymptomatic & 82 & 1.000 & & & 80 & 1.000 & & \\
\hline Symptomatic & 63 & 6.086 & $2.506-14.775$ & $<0.001$ & 61 & 8.372 & $3.000-23.360$ & $<0.001$ \\
\hline
\end{tabular}

${ }^{*}$ Adjusted for gender, age and ethnicity

${ }^{a}$ As defined by 2 or more sexual partners in the past year and infrequent condom use

${ }^{\mathrm{b}}$ OR adjusted for age and ethnicity only as these groups are split by gender

${ }^{\mathrm{c}}$ This category comprises all women, as the number of women who had sex with women in the past year was too small to use as a separate category in this analysis

$\mathrm{OR}=$ odds ratio

MSM = men who have sex with men 


\subsection{Discussion}

The most common reason for seeking healthcare at the SHC among this cohort was experiencing genital symptoms. The length of delay was highly variable, with some people seeking healthcare immediately and others waiting for several months. Almost half of people with symptoms suggestive of an STI waited longer than seven days to seek healthcare. One third of people with symptoms continued to have sex after they first thought they may need to seek healthcare and, as expected, the people that waited longer than seven days to seek healthcare were more likely to have sex while symptomatic. Experiencing symptoms and being a man who has sex with men were both predictive of positive STI diagnosis after adjustment for age, gender and ethnicity.

To the author's knowledge, this is the first study examining healthcare-seeking delay for STI symptoms and sexual behaviour during the delay period in the Australasian region. A previous study $(\mathrm{n}=66)$ did assess time to presentation of patients with genital warts at the Auckland Sexual Health Service but the study did not include data from patients with other symptoms suggestive of an STI and did not assess whether patients continued to have sex while symptomatic (Ireland et al., 2005). Therefore, the current study is the first to document the healthcare-seeking behaviour of New Zealanders for general STI symptoms and the associated risks of onwards transmission.

The proportion of people waiting longer than seven days to seek healthcare for STI symptoms (39.1\% of men and $44.7 \%$ of women) is consistent with that reported in recent studies from other developed countries (Table 2.3). For example, a recent study in the USA found that 38\% of men and 39\% of women with symptoms delayed seeking care for more than seven days (Malek et al., 2013). In the study by Mercer and colleagues in the UK, 45.7\% of GUM clinic patients had been symptomatic for more than seven days before seeking care, although a subsequent study five years later indicated the median length of delay had decreased from seven to three days (Mercer et al., 2007, Mercer et al., 2012).

No statistically significant predictors of delay were identified, however the analysis is likely to have been underpowered due to a small sample size. There were some non-significant associations of delay with female gender, age 25-34 years, Māori ethnicity, having tertiary 
education and attempting self-treatment. A much larger study by Mercer et al. (2007) in the UK also found no predictors of delay. This is contrary to the findings of some earlier studies where females were more likely to delay than males (Leenaars, 1993, Moses et al., 1994, Meyer-Weitz et al., 2000). The influence of age, ethnicity and education on delay is not clear from the literature, with previous studies obtaining different results (Leenaars, 1993, Hook et al., 1997, Gott et al., 1999, Irwin et al., 1999, Meyer-Weitz et al., 2000, Liu et al., 2002, Voeten et al., 2004, Thi Thu et al., 2007, Gerressu et al., 2012).

In total, $26.1 \%$ of men and $35.9 \%$ of women continued to have sex after the onset of symptoms. This is very similar to figures most recently reported from the UK $(25.2 \%$ of men $38.3 \%$ of women) (Mercer et al., 2012). That females are more likely to engage in sex while symptomatic than males is a consistent finding across many studies (Moses et al., 1994, Irwin et al., 1999, Fonck et al., 2002, Voeten et al., 2004), however, the number of participants in this study was too small to reliably test this association.

This study showed that those who delayed healthcare-seeking were more likely to have sex while symptomatic. Although this association has been generally assumed, only a few studies have directly assessed the association (Moses et al., 1994, Irwin et al., 1999, Thi Thu et al., 2007). Of these, two studies have previously reported that delay behaviour is associated with sex while symptomatic (Moses et al., 1994, Thi Thu et al., 2007), but one actually found that sexual activity while symptomatic was associated with attending healthcare quicker (Irwin et al., 1999).

These data agree with many studies showing that key predictors for a positive STI diagnosis were having symptoms and being a man who had sex with men (Fenton et al., 2005a, Mercer et al., 2007, Klovstad et al., 2012). However, it was surprising that some of the other measured variables were not associated with positive STI diagnosis, such as younger age, having had a previous STI diagnosis, being female, or engaging in more risky sexual behaviour. This may reflect that the participants were not a general population sample but rather a SHC sample, who are likely to be at higher-risk of STIs generally which may have affected the association of positive diagnosis with typical risk factors. 
These findings, taken into consideration with those of other studies, suggest that many people continue with their normal sexual behaviour despite noticing STI symptoms. This poses a serious risk for onwards transmission of infection. Of interest, those who had sex with existing partners while symptomatic reported more infrequent condom use. STI prevention tends to focus on casual sexual partners, however these findings suggest that transmission between regular partners might be being underestimated. As waiting longer than seven days to contact health services was associated with having sex after symptom onset, encouraging prompt healthcare-seeking may be one way to mitigate some of this risk.

\subsubsection{Strengths and limitations}

Due to the nature of sexual health research, scientists have to rely on responders being truthful in their recounts of behaviour. One strength of this study is that the data were collected via self-completed questionnaires to reduce the influence of social desirability bias. However, it is possible that the data may still have been influenced by social desirability bias and the participants ability to accurately recall information; the finding that risky sexual behaviour (defined as more than two partners in the past 12 months and infrequent condom use) did not predict STI diagnosis may be evidence of this, as reported in other studies (Turner and Miller, 1997).

The study response rate was high $(73.4 \%)$ and participants were comparable to the wider clinic population meaning the results of the research may be representative of this clinical population. The recruitment phase of the study ran for three months only, resulting in a small sample size which may have led to some analyses being underpowered. However, results were comparable to a similar study with a much larger sample size. It is important to note that although the sample was representative of the overall clinic population, attendees at SHCs are not generally representative of the general population (Catchpole et al., 1997).

Of the symptomatic participants who gave consent to use their STI results, only $39.7 \%$ had a confirmed positive STI diagnosis, with the remainder having a non-STI related cause responsible for their symptoms. However, this study is concerned with healthcare-seeking behaviour for STI symptoms, and the participants in this study were attending a SHC for STI testing because they perceived their symptoms to potentially be an STI, therefore their response to these presenting symptoms is still important, despite the final diagnosis being 
negative for STIs. One limitation is that the questionnaire did not ask which symptoms the participants had experienced or about their severity. Analyses found no difference in delay behaviour between those diagnosed with non-lesion type infections and lesion type infections, although the number of participants included in this analysis was small. It is probable that the type and/or severity of symptoms influences the speed with which an individual seeks healthcare. A previous study of people attending public clinics in Kenya found that women with genital ulcers or lower abdominal pain presented earlier than women with other complaints and men with genital ulcers presented later (Moses et al., 1994), although another study from the Netherlands found no relationship between type of symptoms experienced and delay behaviour (Leenaars, 1993).

This is the first study to quantify delay behaviour for STI testing in New Zealand so provides original data for service planners and providers to work with. These data suggest that public health campaigns should emphasise prompt action for STI symptoms and the need to abstain from sex with all partners until healthcare has been sought and appropriate treatment and/or advice given.

\subsection{Chapter summary and next steps}

This chapter has described the healthcare-seeking behaviour for STI testing of New Zealanders for the first time, and has identified that delay behaviour is associated with an increased risk of sex while symptomatic and thus onwards transmission of infection. It is pertinent to know what factors are influencing this delay behaviour so that they may be addressed by educational campaigns and other interventions. The following chapter attempts to clarify these factors. 


\section{Chapter 4 Barriers to sexually transmitted infection testing in New Zealand: a qualitative study}

\subsection{Chapter Overview}

To understand what might delay or prevent individuals from seeking an STI test, a qualitative research methodology may be employed. This chapter uses data from one-on-one qualitative interviews to uncover the most relevant and important barriers to STI testing. As many STIs are asymptomatic, this study included those with no signs or symptoms of disease as well as those who had experienced symptoms.

Initials used in this chapter: HD = Hayley Denison, AJ = Annemarie Jutel.

\subsection{Abstract}

Objective: To investigate the barriers which prevent or delay people from seeking a sexually transmitted infection (STI) test.

Methods: Qualitative in-depth interviews were conducted with 24 university students, who are a group prone to behaviours putting them at risk of STIs, to understand the factors that had prevented or delayed them from going for an STI test in the past. Resulting data were thematically analysed employing a qualitative content analysis method, and a final set of themes identified.

Results: There were three main types of barrier to STI testing. These were personal (underestimating risk, perceiving STIs as not serious, fear of invasive procedure, self-consciousness in genital examination and being too busy), structural (financial cost of test and clinician attributes and attitude) and social (concern of being stigmatised).

Conclusions: This data will help health providers and policy-makers provide services which minimise barriers and develop effective strategies for improving STI testing rates. The results of this study suggest a holistic approach to encouraging testing is required, including addressing misconceptions, working with healthcare providers to minimise structural barriers and developing initiatives to change social views about STIs. 


\subsection{Introduction}

Sexually transmitted infections (STIs) are a long-standing global health problem with potential serious sequelae including PID, adverse pregnancy outcomes, infertility, rheumatological complications, cancer, organ damage and death (Singh and Romanowski, 1999, Bosch et al., 2002, Haggerty et al., 2010). Although progress towards control of STIs has been made, for example with antiviral therapy for HIV (Pau and George, 2014) and the introduction of the HPV vaccine (Cutts et al., 2007), we are a long way from successfully controlling or eradicating these and other pathogens. Of particular concern are the increasing rates of antimicrobial resistant gonorrhoea (Unemo and Shafer, 2014) and the re-emergence of syphilis (Read et al., 2015).

Young people are disproportionately affected by STIs, with the majority of infections occurring in the 15-24 year old age group (Detels et al., 2011). University students may be especially vulnerable to STIs. Studies indicate that tertiary students engage in multiple highrisk sexual behaviours such as infrequent condom use, sex with multiple partners and casual sex (Welsh et al., 2006, Lewis, 2009). In addition, students typically exhibit high levels of alcohol use (Kypri et al., 2002, Karam et al., 2007), which is associated with high-risk sexual behaviour and STI diagnosis (Aicken et al., 2011b, Connor et al., 2013).

Testing and treating infected individuals limits the harm that STIs may cause to the individual and also reduces the potential for transmission to new partners. Regular STI testing is recommended for sexually active young people in many developed countries (Australasian Society for HIV Medicine, 2014, LeFevre, 2014, NZSHS, 2015a). However, for this to be effective, the at-risk individuals must know and choose to present for testing.

There are many documented barriers to accessing STI prevention and management services. These may be the result of how services are structured but may also be due to fear, embarrassment, stigma, and shame (Morris and Rushwan, 2015). To encourage STI testing, health providers and decision-makers need to understand in detail the barriers which prevent or delay people from testing.

New Zealand provides an interesting case-study to investigate barriers to testing for the following reasons. Firstly, New Zealand has a high incidence rate of chlamydia compared to 
other developed countries including the UK, the USA and Australia (ESR Annual Surveillance Report 2013). Although opportunistic testing by clinicians is encouraged, seeking an STI test is usually an individual decision as in most other developed countries worldwide. New Zealand has a mixed public-private healthcare system, which means STI testing is generally free to those under 22 years old but often attracts a fee for those who are older who seek testing through their GP, which may impact healthcare-seeking for STI. Lastly, there have been no published studies investigating barriers to STI testing in New Zealand for a decade. Here, data is presented from the qualitative arm of a mixed method study investigating healthcare-seeking behaviour for STI in a university population.

\subsection{Methods}

Since the purpose of this research was to seek insight into the attitudes, views and opinions of individuals, a qualitative methodology was chosen. In-depth one-on-one interviews were conducted with students from a university in New Zealand. Participants were recruited by email invitation after taking part in a questionnaire survey at their point of testing, in which they had consented to further contact by the researcher. Participants were eligible to take part if they were university students and had had an STI test in the past 18 months.

Interviews were held in private rooms on the university campus and were conducted by one researcher (HD), with the exception of the first two interviews where a second researcher (AJ) also attended with the participants' permission. Study Information Sheets detailing the background, methods and confidentiality aspects of the study were provided to the participants prior to the scheduled interview date, and further explanation was offered when the participant attended for their interview. Informed, written consent was obtained before the interview commenced and participants were given a grocery voucher of small value at the end of the interview as an expression of thanks for their participation. Ethical approval was granted by the Victoria University of Wellington Human Ethics Committee (ref: 22110) (Appendix D). 
The interview was semi-structured and focused on the factors contributing to the decisionmaking process about going for an STI test. The opening question was "why did you go for an STI test?" Open-ended follow-up questions and probes were used to gain further insight into the factors that discouraged/delayed/prevented attending. An iterative process was employed so that questions were informed by previous interviews and the emerging categories.

Each interview was tape recorded and transcribed verbatim. Resulting data were thematically analysed using NVivo software (version 11), employing a qualitative content analysis method (Schreier, 2012). Data fragments were assigned codes inductively and a constant comparative approach was taken to ensure codes were used consistently. These codes were then grouped together to form categories which became the main themes of the analysis. Two researchers (HD and $\mathrm{AJ}$ ) worked together to identify the final themes and these were tested against the coded transcripts to ensure they were representative of the data. Interviews were continued until theoretical saturation had been reached.

\subsection{Results}

In total, 24 interviews were conducted, at which data saturation was deemed to have been reached. During recruitment, 57 students who had completed the original questionnaire were invited to participate in an interview. Of these, 30 (53\%) responded but five were no longer living in the area and so could not take part in a face-to-face interview, and one was subsequently uncontactable. The participants' ages ranged from 19 to 32 years, and most were undergraduate students $(n=22)$, with two postgraduate students. There were 16 females, seven males and one participant who identified as 'other - genderqueer'. A range of ethnicities were represented: New Zealand European $(n=16)$; New Zealand European/Māori $(n=2)$; New Zealand European/Other $(n=2)$; British $(n=3)$; and Romanian $(n=1)$. The average length of time for each interview (not including the initial introduction to the study and completing of consent forms) was 40 minutes (range $24-62$ minutes). 
From the data, we identified three themes around barriers to STI testing. These included personal barriers (underestimating risk, perceiving STIs as not serious, fear of invasive procedure, self-consciousness in genital examination and being too busy), structural barriers (financial cost of test and clinician attributes and attitude) and finally, barriers related to social condemnation (fear of stigma).

\subsubsection{Personal barriers}

\subsubsection{Underestimating risk}

The majority of participants did not think they were at risk of contracting an STI. Even those who routinely went for STI tests said that they did not expect to have a STI, but just wanted confirmation of a clean bill of health:

I try to go for them quite regularly, I don't ever think I have one I just like to know that I definitely don't. I generally will go if I've had a new partner, even if I don't think that they've got anything. - Interview 7 (female, 21 years)

Personal risk assessments were common. Many participants described the process of weighing up evidence to estimate the possibility of having acquired an STI when making the decision to go for an STI test. Indicators included the number of recent sexual partners, familiarity with those sexual partners, and past sexual behaviour of the sexual partners. As one participant explained when asked whether he would seek an STI test after unprotected sex with a new sexual partner:

It depends who I had a one night stand with. If I knew the person then I probably would put it off more but if I didn't know the person then I'd go, yep. - Interview 16 (male, 21 years)

Several people, both males and females, indicated that they felt they would be able to tell if a sexual partner was likely to have an STI:

It's awful but you can kind of see it from how people act and how they're dressed if they have slept with a lot of people or if they're more reserved etcetera. - Interview 13 (male, 21 years) 
There was suggestion that this risk-assessing was at least in some part influenced by experience of the health professional asking about recent risky sexual behaviour when deciding whether to include a HIV test in the screen. If the health professional thought they were low-risk for HIV, some participants used this as justification for not getting a HIV test at any time, and more generally as confirmation that they were not a high-risk person in terms of their sexual behaviour:

I was quite inquisitive because I haven't had one before so I was asking about "what is this testing for?" and she sort of said it's the more low-level ones and they didn't have to do a blood test unless you thought you might have contracted one of the serious ones like AIDS. But yep, so I haven't had a blood test or really thought there was a need to. Again that would be one of those ones that if you thought there was a need to that's when I'd probably do it but otherwise it seems to be a bit of a hassle. - Interview 13 (male, 21 years)

\subsubsection{Perceiving STIs as not serious}

Although many participants mentioned infertility as a significant concern which encouraged STI testing, there was a general feeling that chlamydia and gonorrhoea were not serious infections. Syphilis and HIV were viewed as serious but so rare that they were not to be a source of worry:

I wasn't concerned if I had [an STI], I didn't think I would have one but if I had one I wasn't concerned because I didn't think it would have been a bad one. If it had been something, it would have been something like chlamydia.Interview 13 (male, 21 years)

The lack of concern about contracting chlamydia or gonorrhoea was at least partly because they are treatable infections: 
Well like I don't panic much about stuff like that because unless it's something very serious you can do something about it once you catch it. Interview 3 (female, 20 years)

A common narrative among the participants was that as they had got older, they had come to realise the seriousness of STIs and thus become more responsible in their sexual and testing behaviour:

If I do have unprotected sex with anyone new I always go and get a check afterwards, I've just done that, but when I was a lot younger I didn't. I was very badly behaved in that respect so I guess the older I get the more aware I am of what's out there and I do try and protect myself as much as possible but sometimes things happen you know. - Interview 2 (female, 23 years)

\subsubsection{Fear of invasive procedure}

Participants reported having very little understanding of what testing would involve prior to their first STI test. Male participants in particular had higher levels of fear and anxiety surrounding both the discussion with the health professional and the test itself. Both male and female participants had assumed that the process would involve a physical examination and invasive procedure. This made them nervous, and several males reported that this was a contributory factor for not seeking an STI test in the past.

Interviewer: 'Was there anything that put you off going for a test?'

Participant: 'It was mainly stories I've heard from people, like about the cotton bud. It made it sound real painful and that it would just be a really terrible time.' - Interview 16 (male, 21 years)

This uncertainty was not aided by different care-providers adopting different protocols for asymptomatic STI testing. Several males reported experiencing a range of testing procedures across different care providers for repeat STI checks, including clinician-taken swabs, firstvoid urine tests and blood tests. As one male participant explained:

Every time there's been a different process to go through to get the check as well. I mean I suppose like an unfamiliarity with what was actually going to 
happen when they... and you get like the stories about it being an even more invasive kind of check and things, and I don't know if I want to subject myself to that. And I know with my experience I've had the urine sample, the swab and blood tests as well and it hasn't been any kind of pattern to what, yeah, and so even now I'm still like "what is going to happen if I do go and get a check?" - Interview 15 (male, 23 years)

Even after having one or several STI tests, participants said they had little understanding of the testing process and of what infections were being tested for:

I didn't really know what it was that was covered by the tests, I don't remember having any knowledge of that and I remember walking out of there and I didn't question, I didn't really ask heaps of questions. I remember walking out of there going "oh I'm glad that they didn't have to stick that thing down my urethra".

- Interview 18 (male, 24 years)

This was especially true with regards to HIV. Around half of participants said they did not know a blood draw was required to do a HIV test or had not known before they'd had a HIV test. Although the participants viewed HIV as a very serious infection, over half had never had a HIV test or were unsure if they had been tested for HIV, despite having had a chlamydia and gonorrhoea test. For example, when asked whether she had ever had a HIV test, one female responded:

Well I've had bloods drawn, I mean a couple of times they have sent me down as part of the STI testing to have blood drawn and get urine samples taken and stuff and those have been analysed and they've come back clear, so they've possibly tested at the same time for HIV but I wouldn't know. - Interview 4 (female, 23 years)

When questioned about whether a blood draw would deter them from testing, only a few participants said that it would. 


\subsubsection{Self-consciousness in genital examination}

Many people reported being extremely nervous before their first STI test. Having to undress and be examined worried many participants, and the general assumption prior to a first STI test was that a physical examination was a necessary part of the process:

It's embarrassing like having to take your pants off and having the doctor like you know fondling your stuff it's like not the best to be honest. Interview 20 (male, 23 years)

Males unanimously preferred the self-taken urine test to being examined by a doctor, but the attitude towards self-swabs among the female participants was mixed. Some were grateful that they would not need to show their genitalia to a health professional, whereas others were concerned that they would not perform the self-swab correctly producing an erroneous result or that it would hurt. As one women who had recently had an STI test explained:

It was a self-swab, like you did it yourself... I like that better because I didn't have to panic about anything else. I didn't have to be like "do I look weird down there?" or "do I like...", you know, something weird. So it was easy, it took like five minutes, not even that, so that was good. - Interview 3 (female, 20)

Another participant explained why she opted for her STI test to be done by the clinician:

I was worried that I'd do it wrong. It's like that when you first use tampons or something you're like "what am I doing?" So that was probably my only concern, or like yeah, just worried that you know, they have explained it to you but maybe you won't do it right and the tests won't be accurate or whatever. - Interview 7 (female, 21 years)

\subsubsection{Too busy}

Another factor that was mentioned in relation to not seeking an STI test or putting off testing was simply being too busy to attend, indicating that it was not a priority:

It's one of those things that you have to just try and keep up with, but you know life gets in the way sometimes. - Interview 22 (female, 30 years) 
Reasons for being too busy included university assignments and tests, paid work and social commitments.

A few participants mentioned the speed of the appointment when explaining why their STI test experience had been good suggesting this is an important factor for some young people:

It's not like it takes a long time, it's just ten minutes in and out, like yep, you just wait for a call and like I reckon if you get tested more often than not you're going to not get a call which is always nice. - Interview 11 (female, 19 years)

A couple of participants also mentioned that it took them a few days to go in for treatment after being contacted by their doctor about their test results because they were too busy to attend straight away. However, both reported avoiding sex until after they had completed their treatment.

\subsubsection{Structural barriers}

\subsubsection{Financial cost of STI test}

The cost of STI testing was considered a potential barrier by the interviewees in the study. The University clinic provides free STI tests to domestic students; therefore the majority of participants in this study had not needed to pay for their tests, which they viewed as conducive to their attending:

Like with my current income and financial position I probably couldn't afford to go as often as I did if it wasn't free. - Interview 10 (female, 21 years)

Many stated that they simply would not have an STI test in the absence of symptoms if they were required to pay, and several pondered aloud what they would do after they graduated and no longer had access to the University clinic. However, it was interesting to note that several interviewees had previously paid for an STI test elsewhere (for example, when away from the University during holidays), usually after engaging in a sexual situation which they perceived to be high-risk. 


\subsubsection{Clinician attributes and attitude}

Although many participants expressed a preference for a same-sex health professional, most said they would not be deterred from having an STI test if a health professional of their preferred gender was not available:

I like that they ask me if I want a male doctor or a female doctor regardless of what my appointment's about. I think that's really good and I don't care if it's a male doctor, well I don't care too much if it's a male doctor that performs those kind of procedures. In general I would prefer it if it was a woman but, for example, if I couldn't have an appointment with a woman doctor until the next week and there was a male doctor who was available that day then I'd go with the male doctor, and if he's not comfortable doing swabs he can get a nurse to do it, but I mean he's a doctor he should be, yep. So yeah the gender of the doctor sometimes has an impact. - Interview 6 (female, 32 years)

Only one (heterosexual male) interviewee spoke about not wanting to be examined by a female doctor because it would be difficult to disassociate that from a sexual situation:

I didn't really want her to have a look because you know, it's just an uncomfortable situation I guess. Just because, I don't know, you have that like male female sort of thing like it feels kind of wrong, the situation, it's hard to displace the fact that they're a female touching your penis from a sexual situation I guess. Whereas if it's a male it's like "ok, this is a doctor, this is fine”. - Interview 13 (male, 21 years)

Men, before their appointments, tended to expect that the nurses would be judgemental or morally disapproving of their behaviour and that the consultation would be awkward. Once having attended, they found they had generally felt comfortable discussing sexual health with the healthcare professional. A few participants did report experiencing a negative attitude during an STI test consultation but this hadn't seemed to have put them off seeking an STI test subsequently. Rather, they said they would simply visit a different clinician next time. 
Other health-service related barriers included: difficulty booking a timely appointment and the inconvenience of having to visit a separate location for a blood draw in some cases. Although around a third of participants described dissatisfaction with having to wait for an available appointment, it did not seem to deter individuals from attending. However, it does have implications for onwards transmission in the meantime, as one participant described:

I feel the waiting times are a little bit ridiculous, it's usually like at least a week which is a little bit stupid because you can do anything within the span of a week. - Interview 9 (female, 27 years)

Concerns about privacy and confidentiality in relation to the health-provider were not frequently mentioned. A few people mentioned not using their family doctor for sexual health issues, but preferring to go to the University clinic. These individuals did not think the doctor would breach their confidentiality, but rather that there was more anonymity with the student clinic because they were less likely to be seen in the waiting room by someone known to their family or whānau:

Yeah with the family GP it is definitely like "oh what if someone sees me, what if someone walks in, a family friend, a school friend", yep. Whereas at uni, it's sort of like, it's a bit different, it's more casual almost. - Interview 24 (male, 20 years)

\subsubsection{Social barriers}

\subsubsection{Concern of being stigmatised}

Stigma emerged as an important factor influencing STI testing behaviour:

STIs are very much stigmatised, I think that's what the big issue is and so I think that's why I don't think people go and get tested as much because they're like "oh my god I've got chlamydia and that's so embarrassing".

- Interview 3 (female, 20 years) 
It was clear in the way many of the participants spoke that they were aware of the stigma associated with STI testing and so would avoid testing or not disclose their testing activities widely:

They might think that you either have an STI or that you might be considered like a little bit of a slut because you've been sleeping with other people and not using protection and stuff like that. So I wouldn't tell anybody but I guess with your close friends you know they probably know that it's just like routine, yeah. - Interview 21 (female, 21 years)

Though some people actively challenged this stigma:

It's no different to any other sort of check-up when you go to the doctors. I think if anyone come up to me about why are you getting a check-up, do you know what I mean, it's like well I'm the one's that's looking after myself and making sure that my partner's healthy as well. - Interview 24 (male, 20 years)

It should be noted that the stigma discussed by the participants was perceived stigma, rather than actual stigma, as very few had stories of actually being discriminated against due to STI testing or being diagnosed with an STI. The opinions that most people feared were those of ex, current or future partners, not those of friends or acquaintances, and one of the most fearinducing consequences of having contracted an STI was the need to inform current and recent sexual partners.

It was encouraging to note that a small number of participants felt that stigma about STIs is reducing:

I think like a lot of the stigmas around STIs are disappearing and that's really cool. So I think like everyone's kind of more aware that it's a lot easier to contract it's not that you have to sleep with a hundred different people to get one. - Interview 2 (female, 23 years) 


\subsection{Discussion}

This study found several barriers to STI testing which can be broadly grouped into three overall themes: personal barriers, structural barriers and social barriers.

Most participants did not feel they were at risk of contracting STIs and even after deciding to have an STI test, very few believed they would receive a positive STI test result. This lack of risk perception has been reported in other studies (Ethier et al., 2003, Abel and Brunton, 2005, Zakher and Kang, 2008, Wolfers et al., 2011, Oliver de Visser and O'Neill, 2013). Participants generally felt that they could judge for themselves whether a sexual partner or potential partner was 'clean' or not. Participants would look for indicators of 'dirtiness', including the way individuals dressed and acted. This attitude reflected that found among male students in a UK University who maintained that they could avoid chlamydia by being wary of 'risky types' of women (Chaudhary et al., 2008). A sense of invulnerability is potentially dangerous as people will not take the necessary steps to protect themselves if they do not feel at risk. Making people aware of their own risk and the severity of STIs may be one way to encourage STI testing in this population.

Many people in this study reported that they had never had an HIV test even if they had been tested for chlamydia and gonorrhoea, though the findings suggest some people may be unsure. Similar findings have been reported from other studies of this age-group and it is suggested this is because young people have not been exposed to the high-volume of public health messages about HIV as previous generations were during the initial stages of the epidemic in the 1980s and 90s, and they have seen less HIV-associated mortality due to improvements in ART (Beltzer et al., 2013).

Like other studies, we found that prior to a first test, males expected the STI test to involve a urethral swab and be a painful experience (Blake et al., 2003, Chaudhary et al., 2008, Shoveller et al., 2010, Latreille et al., 2014). Although this expectation had initially prevented some males from attending for an STI test, most participants reported that these fears were alleviated at their first consultation and they became more pro-active about reattending for STI testing. Current laboratory diagnostic techniques allow for urine tests to be used for $C$. trachomatis and $N$. gonorrhoeae testing in asymptomatic men. However, several 
males in this study reported experiencing different types of tests on different occasions (even when asymptomatic) which led to confusion and renewed anxiety about what to expect. This suggests that standardising care so that only urine tests are used for asymptomatic males may result in less confusion and increased healthcare-seeking.

Being too busy was a reason for some participants to put off seeking an STI test. This finding indicates that STI testing isn't a priority for these individuals and links with the sub-themes of underestimating risk or perceiving STIs as not serious. Offering testing as part of a clinical consultation for another reason may be one method of circumnavigating this issue, as the individual has already attended for a health issue they do see as a priority. Opportunistic testing for chlamydia is recommended in New Zealand for all sexually active people aged under 25 (NZSHS, 2015a). The findings of this study support that recommendation.

The cost of STI testing was frequently mentioned as a barrier. However, there seemed to be a dichotomy between regular low-risk testing and testing because individuals perceived there to be a problem, which was seen as worth paying a fee. Given that most individuals fail to accurately assess their risk, this has serious implications for identifying untreated infection in the community. Previous research in New Zealand showed that introducing free sexual health GP consultations for under-25 year olds significantly increased the number of people attending for an STI test, as well as the number of chlamydia infections diagnosed (Morgan and Haar, 2009). Health providers need to be aware that the fees associated with STI testing may reduce regular check-ups in the absence of symptoms, and aim to provide free services as widely as possible. Additional structural barriers to STI testing included difficulty booking a timely appointment, having a separate location for blood draws, and the gender of the health professional. Although these factors were offered as barriers, in many cases the participants had still chosen to present for STI testing. Patient-doctor confidentiality was not a big concern for the individuals in this study, which is contrary to findings from several studies of young people in the USA and Australia (Barth et al., 2002, Blake et al., 2003, Ewert et al., 2016). This finding may represent a cultural difference, or may be due our sample being drawn from a clinical site thus biasing the sample towards people who have confidence in the confidentiality of the patient-doctor relationship. 
Similar to other studies (Barth et al., 2002, Balfe et al., 2010c, Richardson et al., 2010), fear of stigmatisation seemed to be an important factor preventing seeking STI testing for these participants. Stigma may negatively affect healthcare-seeking behaviour for STI testing because seeking an STI test suggests that socially undesirable behaviour has occurred (Lichtenstein, 2003) and this presents a threat to peoples' social identities (Balfe et al., 2010b). Male participants in this study appeared to be more concerned about being stigmatised if they were seen to be requesting an STI test than female participants.

Barriers to STI testing have not been well studied in New Zealand. Rose et al. (2008) carried out focus groups with 16 to 24 year olds and health-professionals in order to find ways to encourage chlamydia testing. Several reasons why young New Zealanders would not seek chlamydia testing were identified including fear, stigma, denial of personal risk and lack of knowledge, which reflect some of the barriers found in the current study. This suggests that there has been little change in the perceived barriers to STI testing over the past decade in New Zealand, indicating that more needs to be done to alleviate these obstacles to testing.

There are some potential limitations of this study. University students are not representative of all young people and barriers to testing may be different in non-student populations. In addition, those that agreed to be interviewed may have more of an interest in this topic and so may have different opinions and experiences to those who declined to participate. While there was some ethnic diversity within the study sample, only two participants identified as Māori and no participant identified as Pacific. The reasons for this are unclear, though possible explanations include that study information was provided in English rather than in Te Reo Māori or other languages, or that cultural differences in the acceptability of discussing sexual issues with a stranger may have deterred participation. Māori and Pacific peoples experience a higher burden of infection than New Zealand Europeans (ESR Annual Surveillance Report 2013, 2014). Unfortunately, the low number of Māori and Pacific peoples in this study limits the ability to draw any valid conclusions about specific issues with regards to healthcare-seeking behaviour for STI testing for these people. Understanding the specific needs and barriers of Māori and Pacific peoples is necessary to enact evidencebased changes leading to more equitable sexual health within New Zealand. 
It is important to note that these individuals had access to an on-campus health service which offered free STI tests to domestic students which may have reduced some of the usual barriers to testing. In addition, the participants in this study were recruited through a clinical site and all had experienced an STI test at least once, therefore the views of those that have never had an STI test are not represented in these data. These may be the people for whom barriers exert a particularly strong effect, or there may be different barriers for the people not represented in this study. That said, Richardson et al. (2010) interviewed 14 young people aged 16 to 24 years who had turned down chlamydia testing as part of the chlamydia screening programme in England and identified similar themes: stigma, embarrassment, perception of risk and beliefs of what the test involves.

This paper provides information on what prevents or discourages STI testing in a New Zealand population. Such information is helpful in the generation of interventions to promote STI testing uptake. Traditionally efforts have targeted individual level factors associated with STI risk, but this study supports the idea that to be effective, they will also need to address higher-level factors such as social and cultural conditions that also influence healthcareseeking behaviours (DiClemente et al., 2007). With this in mind, a holistic approach needs to be taken, targeting individual-level factors such as misconceptions, working with healthcare providers to minimise structural barriers, and developing polices and initiatives designed to change social beliefs about STIs.

\subsection{Chapter summary and next steps}

This chapter has identified several potential barriers to testing, many of which are modifiable through service provision (reducing the cost of STI tests, offering non-invasive tests) or educational campaigns (stressing the need to regularly test and explaining the testing process). Others will be more challenging to address, such as reducing the social stigma attached to STIs and associatively, STI testing.

To effectively improve STI testing among individuals at risk, not only do barriers need to be removed, but motivators need to be amplified. Understanding the reasons which drive people 
to seek STI testing will help to design methods encouraging this behaviour. Uncovering the factors that positively influence STI testing is the objective of the following chapter. 


\section{Chapter 5 "It's like brushing your teeth... only every few months": University students' reasons for STI testing}

\subsection{Chapter Overview}

The previous chapter identified barriers to STI testing among a sample of university students in New Zealand. This next chapter uses data from the same qualitative interviews to uncover what the motivators for testing were among this group. This information will add more depth to our understanding about the decision-making process involved in seeking an STI test, as well as provide possible avenues for encouraging STI testing behaviour.

Initials used in this chapter: $\mathrm{HD}=$ Hayley Denison, AJ = Annemarie Jutel.

\subsection{Abstract}

Background: Untreated STIs can lead to serious health complications, increase susceptibility to contracting further STIs including HIV, and can be transmitted to others. The early diagnosis and treatment of STIs is therefore central to comprehensive STI management and prevention, but this relies on those at risk of STIs presenting for testing. In order to understand STI testing behaviours with a view to improvement, this study aimed to elucidate the reasons why people at risk of STIs seek testing.

Methods: Qualitative in-depth interviews were conducted with 24 university students who had recently had an STI test. Resulting data were thematically analysed employing a qualitative content analysis method to produce a final set of themes.

Results: The data revealed five drivers for testing: crisis, partners, clinicians, routines, and previous knowledge. The final driver, previous knowledge, intersected with the previous four, particularly in relation to routines. Many participants acknowledged that the more they knew about STIs the more likely they were to undertake routine tests. However, at the same time, many participants felt they did not have a good knowledge base and that their school-based sex education had been lacking.

Conclusions: This study highlights important drivers for STI testing, which may aid the design of public health campaigns. It also underlines that school-based education could provide stronger foundations with regards to STIs and their prevention. 


\subsection{Introduction}

Sexually transmitted infections (STIs) are a serious public health problem, with nearly a million people acquiring an STI every day (World Health Organization). Young people are disproportionally affected by STIs. For example, in New Zealand, laboratory surveillance data showed that $83 \%$ of chlamydia cases and $73 \%$ of gonorrhoea cases in 2014 were in people aged between 15 and 29 years (ESR Annual Surveillance Report 2014). There are several reasons for this, including both biological and behavioural factors (Lee et al., 2006, Chinsembu, 2009, Slater and Robinson, 2014). University students are considered particularly vulnerable to STIs due to engaging in multiple high-risk sexual behaviours such as infrequent condom use, sex with multiple partners and casual sex (Lewis, 2009). Heavy alcohol use is common among the student population, and this has been shown to be associated with sexual risk taking and STI diagnosis (Deogan et al., 2012, Connor et al., 2013, Chanakira et al., 2014).

Untreated STIs can have serious sequelae including PID, infertility and adverse pregnancy outcomes (Idahl et al., 2004, Haggerty et al., 2010). Furthermore, having an STI increases susceptibility to contracting further STIs, including HIV (Ward and Ronn, 2010). Nondiagnosis of any STI also increases the likelihood that an infected individual will transmit the infection to a sexual partner. Therefore, the early diagnosis and treatment of STIs is central to comprehensive STI management and prevention.

Understanding the views and behaviours of sexually active young people towards STI testing may enable us to tailor sexual health programmes and interventions to encourage presentation for STI testing. While the barriers to STI testing have been well documented, the facilitators remain poorly researched (Yeung et al., 2015). Investigating this in New Zealand is of considerable relevance, as infection rates are high by international standards, and testing coverage rates are below the level mathematical modelling has suggested is required to decrease chlamydia prevalence (ESR Annual Surveillance Report 2014). There is also no systematic population screening programme in New Zealand, such as the National Chlamydia Screening Programme (NCSP) in England, so individuals are responsible for seeking a STI test. In order to better understand the factors influencing STI testing 
behaviours, university students who had recently had an STI test were recruited to a mixed methods study. Their reasons for requesting a test and the factors involved in making a decision to be tested were explored. In this paper, we report the findings of the qualitative branch of the study, in which we investigated the personal reasons for getting an STI test.

\subsection{Methods}

\subsubsection{Participants and Recruitment}

The participants were students from a New Zealand university in Wellington who had attended the university medical clinic for an STI test and had completed a questionnaire about their visit. An invitation to participate in a follow-up interview was emailed to those who had agreed to be contacted. Participants were given a grocery voucher of small value as an expression of thanks for their participation. Ethical approval was granted by the Victoria University of Wellington Human Ethics Committee (ref: 22110) (Appendix D).

We used purposeful sampling to capture a range of motivations for testing. This was achieved by selecting people on the basis of their answer to the multi-choice question 'Why did you decide to go for an STI test?' in the initial questionnaire. We aimed to include participants who voiced a variety of reasons for testing to maximise the phenomenal variation (Sandelowski, 1995). As the study progressed, categories began to emerge in relation to factors influencing STI testing, and the sampling strategy was then adjusted to saturate these categories and explain more of the theory (theoretical sampling). This methodology was informed by the writings of Patton (1990), Sandelowski (1995) and Coyne (1997). Participants were sought until the point of data saturation; this was deemed to have been achieved when no new reasons for testing were proposed by participants in the interviews.

\subsubsection{Data Collection}

Interviews were held in private rooms on the university campus and were conducted by one researcher (HD), with the exception of the first two interviews where a second researcher (AJ) also attended with the participants' permission. The researcher(s) gave a brief 
explanation of the study to the interviewee, reassured them of confidentiality, and clarified that the interview could be stopped at any time they wished. Informed, written consent was obtained before the interview commenced.

The interview was semi-structured and focused on the factors contributing to the decisionmaking process about going for an STI test. The opening question was 'why did you go for an STI test?' Follow-up questions and probes were asked including: reasons for attending; factors that facilitated/encouraged attending; reasons for any delay; and factors that discouraged/delayed attending (see Table 5.1). Open-ended questions were used to allow the respondents to elaborate on their experience or attitudes. An iterative process was employed so that questions were informed by previous interviews and the emerging categories.

\section{Table 5.1. Interview Guide}

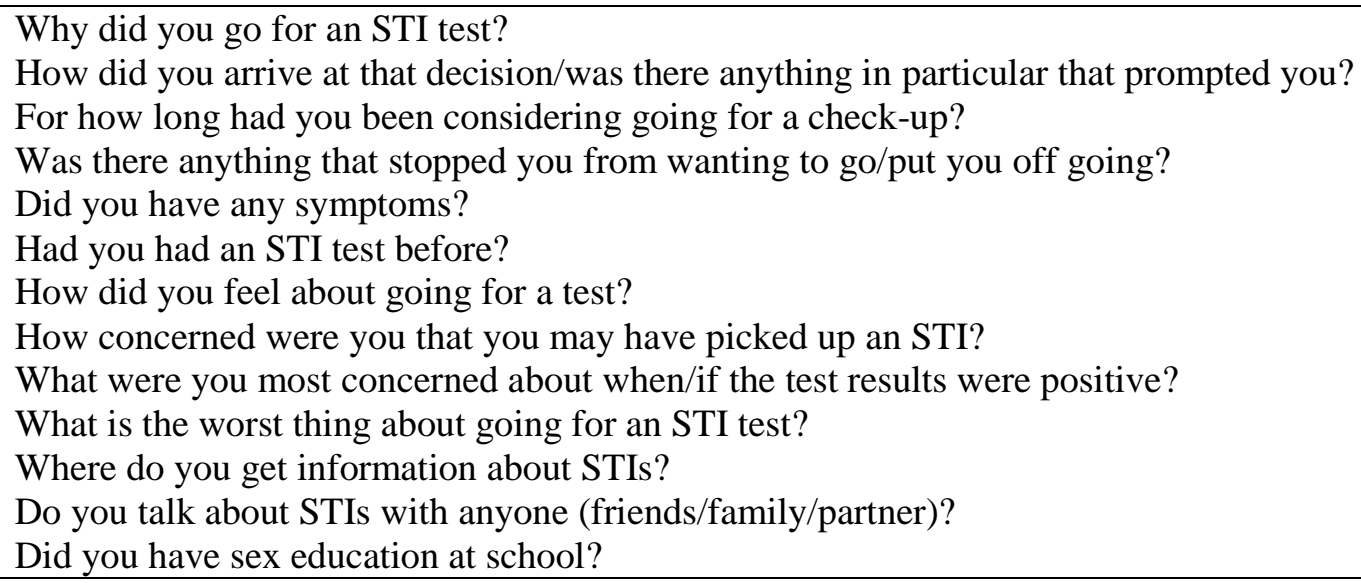

The interviews were tape recorded and transcribed verbatim. Shortly after each interview, the interviewing researcher (HD) would explain and discuss the main points arising in the interview with a second researcher $(\mathrm{AJ})$ to aid interpretation of the content of the interview. A summary of the main points was prepared and sent to the participant for review to confirm this accurately captured their decision-making process and views. Immersion in the data took place throughout the interviewing period and after, using familiarisation techniques including: listening to the audio of each interview multiple times; reading transcripts repeatedly; and going over observational notes. 


\subsubsection{Analysis}

Transcripts were first reviewed alongside the audio recordings to check for errors and omissions. The data were analysed using NVivo software (version 11), employing a qualitative content analysis method (Schreier, 2012). Codes were drawn directly from the data and assigned to the transcripts inductively. A constant comparative approach was taken to ensure coding categories were used consistently. The coded transcripts were then reviewed to determine the emerging themes, and two of the researchers (HD and $\mathrm{AJ}$ ) took part in regular discussions in which further theorising and making connections between coding categories took place to identify the major themes. Emerging hypotheses were tested against the coded transcript data before the major themes were finalised.

\subsection{Results}

In total, we approached 57 students who had completed the questionnaire to participate in interviews. Of these, 30 responded but five were no longer living in Wellington and so could not take part in a face-to-face interview, and one was subsequently uncontactable.

Recruitment took place concurrently with interviews to allow sampling and assessment of data saturation. Saturation was reached after 24 interviews. The characteristics of participants are summarised in Table 5.2. Participants were not asked directly about their sexual preference behaviour/sexuality, although discussions involving sexual preference did take place during some of the interviews. Questionnaire data revealed bisexual behaviour was common among the women (5/16) and less so among the men (1/7) in this sample. 
Table 5.2. Demographic profile of participants

\begin{tabular}{lll}
\hline Demographics & $\boldsymbol{n}$ & $\mathbf{\%}$ \\
\hline Gender & & \\
$\quad$ Female & 16 & 67 \\
Male & 7 & 29 \\
$\quad$ Genderqueer & 1 & 4 \\
& & \\
Age (years) & & \\
$\quad$ Mean (range) & 23 & $(19-32)$ \\
& & \\
Ethnicity & & \\
$\quad$ New Zealand European & 16 & 67 \\
$\quad$ New Zealand European/Māori & 2 & 8 \\
$\quad$ New Zealand European/Other & 2 & 8 \\
$\quad$ British & 3 & 12 \\
$\quad$ Other & 1 & 4 \\
& & \\
Educational level & & \\
$\quad$ Undergraduate & 22 & 92 \\
$\quad$ Postgraduate & 2 & 8 \\
\hline
\end{tabular}

From the data, we identified five 'drivers' for testing. 'Drivers' were common beliefs or situations that were likely to incite a participant to seek an STI test. They included: crisis, partners, clinicians, and routines. The final driver, previous knowledge, intersected with the other four, but most prominently in relation to routine-driven testing, whereby better knowledge encouraged more regular testing. However, we discuss this knowledge as a separate theme below, because the lack of, and/or the desire for, knowledge emerged as relevant to the other drivers too. 


\subsubsection{Crisis}

For some, the first STI test was prompted by an event the participant experienced as a crisis, such as developing symptoms, finding out that a previous sexual partner had been diagnosed with an STI or having engaged in a sexual encounter which they perceived to be high-risk. A male participant explained:

My ex-girlfriend came to me like two or three months later after we'd broken up and said "look I've got herpes" or something like that and I had a terrible terrible moment, I said "oh my god" you know? I got myself checked out and they were like "no you haven't got anything”. - Interview 24 (male, 20 years)

Another participant reflected on a situation that had happened some years before:

I had an HIV scare when I was about 18. I was with this guy and I broke up with him, and then after that somebody informed me that someone he had been sleeping with before was HIV positive, and I had not used protection with that guy. So I went and got checked and it was fine but that really scared me. - Interview 6 (female, 32 years)

Some people reported having been sexually active for several years before ever getting a test. Often a crisis moment would occur which would force a test, after which the individual would then proceed to have testing much more regularly. This change in behaviour was attributed to the realisation that STIs presented a real risk and was facilitated by a new understanding that having an STI test was not as arduous or traumatic as expected:

My mind-set has changed, like it's not that bad getting tested, it doesn't take that long. It's not hard, I'd rather go and get tested and know I'm clean than not be tested and have something. That's why I get tested and if I have something it's just a course of antibiotics or whatever then I'm clean. - Interview 16 (male 21 years) 


\subsubsection{Partners}

A second driver was the perspective of, or advice from, sexual partners. Male participants, in particular, were motivated to seek testing after discussion with their female sexual partners. Often, the individual had been asked to get tested by a current girlfriend:

I'd had a few partners but not many and always used condoms and everything and so that was the first time that someone had said to me "when was the last time you had an STI test?" I was like "never." She was like "you should get tested". - Interview 18 (male, 24 years)

Another reported that his girlfriend had withheld sex until he was tested:

I mean it just kind of came up about our past and that she wanted me to get checked and her to get checked before we got into anything serious really so we didn't catch anything from either one. Yeah. ....She kind of used it like a threat that if I didn't go then we couldn't have sex until I got checked.

- Interview 16 (male 21 years)

One female participant had a similar stance:

Well we're all adults and it's a fairly common issue, and I'll just be like "I'm sorry but I don't feel comfortable having sex with you until we both get checked". - Interview 9 (female, 27 years)

Another male participant, who only discussed sexual partners as being female, explained how female opinion of his STI status was more of a concern than the opinion of his male friends. Here he describes how in a group situation, a female friend asked him if he had ever had an STI test. This prompted him to go for his first test:

I guess that's a good point as well that it was a girl that asked me if I'd had an STI test because I'd be more worried what they would think of me than a guy I imagine. If just the guys were like "oh yeah STI check" it wouldn't be as big an issue to me. And also the girl that asked me, I was dating one of her friends at the time so that was probably also an impetus. - Interview 13 (male 21 years) 


\subsubsection{Clinicians}

One other important driver to having an STI test reported by the participants was the opportunistic advice of a doctor or nurse who suggested an STI test while the individual was attending the clinic for a different reason. This often occurred when attending for sexual health related matters, such as a cervical smear. As one women explained:

The first one I think was when I was vaguely scared that I was pregnant and so I got an STI check kind of at the same time just because I was there and the other time I think I was getting a smear and I also got offered an STI check I think. - Interview 23 (female, 24 years)

For another, the disclosure of unprotected sex appeared to have prompted the clinician to advise an STI test:

I told the doctor that I had a serious boyfriend and we don't use protection at all and she panicked for me and said "we're just going to test you".

- Interview 8 (female, 29 years)

This driver was not mentioned by any of the males in study. This may have been due to the smaller number of male participants. Though one female participant, whose boyfriend had never tested, believed that males test less often, partly because they attend the doctor less and so do not get offered testing as regularly as women:

I think it's partly because boys don't need to go in as often as girls because we talk about contraception with the doctors and which you need to go in anyway so there's more of an opportunity for women to talk about it, whereas for men they don't really go in anyway and they probably wouldn't really want to book an appointment just for that. - Interview 21 (female, 21 years)

\subsubsection{Routines}

The fourth driver to have STI testing was routine. Some participants went for STI tests regularly, as part of their routine health care. Those who used this reason for testing didn't go for a test because they thought they had an STI. Rather, they were motivated by wanting 'peace of mind' about their own health and/or to know they wouldn't be passing an infection 
on to their partner(s). This rationale was held mainly, but not exclusively, by female participants. These participants reported either being tested at regular intervals, or testing either at the end of a sexual relationship or the beginning of a new one. While they might have had a partner in mind, they made their decisions autonomously without discussion with partners or friends. As one participant reported:

Being single, I just, it's something that I do on a regular basis. I had no reason to suspect that I had anything I didn't have any symptoms or anything like that it was just ... if you're going to be single and be sexually active you have to do that. I mean well that's the way that I see it you should do that in order to make sure that you're healthy, for peace of mind and yep it's like brushing your teeth but every few months. - Interview 22 (female, 30 years)

A female participant discussed her rationale for always testing at the end of a relationship, whether she had used condoms or not:

I feel like it's almost like a clean slate, like you know if you have a breakup you change your hair colour or get a haircut, I go and get an STI test, because I'm like okay I just want to make sure that it's all good, that I'm sweet and everything else and then I can move on without knowing I've done something that would affect someone else, because I'd hate that. - Interview 3 (female, 20 years)

Believing that an STI test was just another part of routine health maintenance was demonstrated by those who grouped testing with other health issues for which they sought medical advice. One woman explained:

And you usually try, you like try and roll four issues into the same one. Like the last one I went to I was like "okay so I've got this thing on my leg and I think it's a blood clot, I'd also like an STI check and I have the 'flu, I'd like some antibiotics and I also would like to quit smoking so we can talk about some anti-smoking things please”. Because you just have to do it because otherwise you won't get in there for another like three or four weeks, you've got to like just ball it in kind of thing. - Interview 2 (female, 23 years). 
A clinical attitude towards STIs seemed to facilitate STI testing; unsurprisingly this view was commonly exhibited by those who had come from a family where sexual health was discussed openly and/or had a parent who was a health-care professional. One participant explained:

I come from a family of medical practitioners so for us it's not, it's a human body it's another part of the body. It's like getting your ear infected, like an infection. - Interview 9 (female, 27 years).

Another pointed out:

I think because my mum's a midwife and like that sort of thing for her is just like common occurrence like she looks at vaginas all day. It's kind of like I've always had that in my head so it's not embarrassing thing to go to the doctors to get done, but I think with lots of people it would be. - Interview 11 (female, 19 years)

\subsubsection{Previous knowledge}

Knowledge about STIs and their potential long-term consequences motivated many of the participants to seek STI testing. For example, one participant explained that realising how prevalent STIs are caused him to consider going for a test:

In first year we were talking, one of the guys talked about how he'd had one before and so I was like "oh maybe I should actually think about them now, it's not just kind of this very rare thing that only occasionally happens".

- Interview 16 (male, 21 years)

Good knowledge of STIs, including the long-term health consequences of infection, was common among those who were routine testers. For example, a regular tester explained:

It is important that you do it and I don't think people realise how important it is. Like they'll be "oh you can just take a pill and it gets rid of it" but they don't realise if you don't catch it straightaway it can cause damage and all that kind of stuff. So I'm very aware of that. - Interview 3 (female, 20 years) 
About a third of participants reported being aware that STIs such as chlamydia were common. Their awareness often came from friends who had been diagnosed with an STI. As one woman described:

I had two friends who had it at high school like three maybe all throughout, but like these last two years there's been heaps of people that have had it. I mean it's probably because people are having more sex but yeah that probably motivates me to get a check-up more because I realise that people aren't careful and they don't care. - Interview 1 (female, 19 years)

Perhaps not surprisingly, a lack of knowledge was reported as a reason for not previously attending for an STI test. This pre-test ignorance seemed to be characteristic of those whose testing had been partner- or crisis-driven:

I never really thought about it, about the risks of getting an STI or the consequences of having one or just the chances of getting one... it just hadn't occurred to me that I could get one. It was kind of that invincible kind of mentality that it can't happen to me. I didn't know the consequences of certain STIs like what they actually do to you. I thought they were just annoying little things, like I wasn't really educated about them. - Interview 16 (male, 21 years)

However, for one participant, a lack of knowledge was in fact the reason for regular testing: I'm a bit lacking in knowledge about STIs and stuff so I don't, I think that's what caused me to be a lot more sort of aware and like nervous about that sort of thing and strict with myself with getting regular checks because it's more of an unknown factor for me. - Interview 20 (male, 23 years)

Many participants felt that knowledge was the key to safer behaviour and it was common for participants to express that they wanted to be more knowledgeable, especially among those whose test had been crisis-driven. A large number of participants felt that the sex education they had received at school had not adequately prepared them for the potential dangers associated with sexual relationships. Some were quite angry that their sex education had not 
been comprehensive enough or had been designed to scare rather than inform. Female participants were especially passionate about this. One woman described the sex education she received as school:

They would just show us like slideshows of like the worst possible looking STI that you could possibly find on the internet and go "this is what's going to happen if you don't use condoms" and then like I didn't realise you could get treated for the majority of them I just thought "oh crap now I've got it I'm chuffed for life", because they didn't really explain it. - Interview 3 (female, 20 years).

Many males and some females felt that sex education had not been relevant to them at the age they received it at school, as they had not begun to have sex yet, but acknowledged that it was necessary for those who had started having sex. Many could not recall what was included in their sex education and expressed a desire to have had sex education sessions at older ages as well, including at university. As one male explained when asked whether he'd received sex education at school:

Yes but at high school it wasn't taken seriously I guess. Just how it was taught, I guess we were taught it quite young. I think I was taught it in year 9 or year 10 so it was before I started having sex. It felt kind of, I didn't relate to it at the time but I didn't think it would ever kind of be a problem, I didn't retain the information. - Interview 16 (male, 21 years)

No participant mentioned having been told about the necessity of STI testing in the absence of symptoms, or about the STI testing process as part of their formal school sex education.

\subsection{Discussion}

Five drivers for testing were identified from the data, including crisis, partners, clinicians, routines and knowledge. These pathways to care appeared to be subject to gender differences, for example, clinician driven testing and routine driven testing were mainly 
mentioned by females and partner driven testing was mainly mentioned by the males. Crisisand knowledge-driven testing did not appear to be as gendered as the other drivers.

In a similar study of young people in Ireland, there were four main reasons for seeking an STI test; having had unprotected sex, developing symptoms, being required to do so by an employer or for a visa, or reaching a transitional moment in life (Balfe and Brugha, 2009). Examples of transitional moments were the end of a risky relationship or the beginning of a new intimate relationship, which were also offered as reasons for testing in the current study. However, we found that if people tested in these situations, they did so as a matter of routine every time a relationship ended or started. Testing because of employer or visa requirement was not reported in the current study, which is likely a reflection of the participants being university students.

Many of the participants in this study presented as heterosexual, even though some participants described having sex with same-sex partners. It is possible that we would have identified different drivers for testing in groups of different sexual preference, or if we had asked them more explicitly about their sexual preferences. However, the results of the current study are broadly similar to those found in a USA study of MSM (Mimiaga et al., 2007), where three categories of reason for seeking an STI test were identified: event-driven (experiencing symptoms or illness, having engaged in 'risky' sex, or finding out a partner was infected); prevention-driven (being tested as a matter of routine care); and socially driven (entering a new relationship, being encouraged by others, and/or following peer norms).

For males who only discussed sexual partners as being female, being influenced by a female who was a potential sexual partner (or 'gatekeeper') was highly persuasive, and was often the driver for their first STI test. In this way, the women were able to protect their own health by refusing to engage in sexual intercourse until their partner had been tested. There was also an implication that it was the testing, rather than the result per se, that mattered to the women. Perhaps this was viewed as an opportunity to educate or impress the notion of sexual health responsibility, which many of the women felt that their male counterparts lacked. 
Opportunistic testing, where clinicians offer a test to an individual who is attending the health service for another reason, was acceptable to people in this group as has been shown in other studies (Heritage and Jones, 2008, Zakher and Kang, 2008, Hogan et al., 2010). In New Zealand, the Ministry of Health Chlamydia management guidelines (2008) recommends opportunistic testing for chlamydia for all sexually active people aged under 25 years who fulfil risk criteria (such as new partner(s) or inconsistent condom use). Opportunistic testing may be especially effective in those who don't perceive themselves at risk so would not seek a test on their own, or for those who are nervous or embarrassed about speaking about sexual health and might have difficulty bringing it up with a doctor.

Hidden patient agendas are common in the general practice setting (Barsky, 1981, Hunziker et al., 2011), and trainee primary care physicians are often taught methods to uncover these (Silverman, 2005). Patients may not always be forthcoming about STI concerns and physicians should be particularly aware of this in young people due to their elevated risk of STI. Pilot studies to increase opportunistic chlamydia screening in general practice in New Zealand have been shown to be effective at increasing rates of testing (Lawton et al., 2010, Azariah et al., 2013). However, opportunistic testing will be biased towards women as men tend to attend the doctor less (Dickson et al., 1998), therefore alternative educational and/or testing opportunities need to be explored to improve levels of male testing. Importantly, this study showed that once a male had attended for an STI test a first time he would continue to have tests thereafter, indicating that interventions targeted towards never-testers should be prioritised.

The interviewees wanted to be more knowledgeable about STIs, and felt that better knowledge would encourage testing, a finding echoed in other studies (Blake et al., 2003, Hall et al., 2014, Litras et al., 2015). Poor knowledge can translate into misconceptions about the infection and about screening (Balfe et al., 2012).

The perception that people can be 'clean' (uninfected and pure) or 'dirty' (infected and deviant) seems to have been reinforced during high school sex education, where scare tactics promoted STIs as grossly symptomatic and only occurring in those who are promiscuous. Associating a condition with a particular type of person increases stigmatisation, especially when beheld as punishment for a moral transgression (Goffman, 1963). In this way, STIs are 
used as an instrument of social control (Burris, 2008). We found that when individuals became aware that a personal contact such as a friend or housemate had contracted an STI, it altered their negative view of STIs, possibly because the friend contradicted the stereotype of an infected individual (Foster and Byers, 2008). We found that upon realising that STIs are common and happen to people of all social standings, many young people felt angry that their sex education had been misleading and that they had not been made aware of the risks. This echoes the findings from Allen's 2005 study involving 1180 students in 15 New Zealand schools, in which students were critical of the way potential dangers of sexual activity dominated programme content and approaches (Allen, 2005). Dissatisfaction with sex education has also been reported in other studies from around the world (Henning et al., 2007, Ekstrand et al., 2011, Javadnoori et al., 2012).

Several of the male respondents and a few of the females commented that sex education had occurred too early for them as they were not yet having sex. As it did not seem relevant at the time, they either did not pay attention or had forgotten what was taught, a finding reflected in a recent Australian study of young males (Litras et al., 2015). In New Zealand, the median age of first sex is 16 years old for females and 17 years for males (Psutka et al., 2012). Under the National Administration Guidelines, schools in New Zealand are required to teach sexuality education within a broader programme up to and including Year 10 (approximately age 14). The recently updated Sexuality Education guidelines from the Ministry of Education however, recommended that students in years 11-13 (approximately ages 15-18) also engage in sexuality education (Ministry of Education, 2015), and the results of this study would support that. It may also be beneficial for sexuality education refresher to be included at university, a time of sexual exploration for many young people. This has recently been suggested by researchers in Australia as well, following a similar study among male students in Victoria (Ewert et al., 2016).

The participants in this study believed testing needed to be normalised. This would, however, require a change in the way society shames people diagnosed with an STI, so that people are more comfortable talking about them. The process is circular in that a reduction in stigma can only be achieved by talking about STIs. This study indicated that open and honest discussion led to responsible testing behaviour, whether this occurred in the family home growing up, or 
amongst friends at a later age. Often, discovering that a peer had contracted an STI triggered the realisation that STIs were common and a serious risk and thus influenced them to be tested. However, studies have shown that people may not disclose their STI if they perceive that doing so will have negative consequences for their relationship with others (Balfe and Brugha, 2010).

The reasons why young people in New Zealand go for STI testing has not been well studied. Rose et al. (2008) conducted focus groups in which they asked young New Zealanders what they thought would encourage chlamydia test uptake. Some of the suggestions included better education and normalisation of testing, which was reflected in this study. The study was conducted almost a decade before this one, yet it seems this call has not been answered.

There are some limitations to the current study. The participants were recruited through a clinical site and all had had an STI test at least once. Although we explored individuals' attitudes and thoughts prior to their first test, the experience of having a test may have influenced their recall of these. In addition, all participants were drawn from the university clinic, which is likely to have been more accessible than a general GP practice would be for non-students, given it was located on campus and is youth-centred. Being diagnosed with an STI is an emotionally charged event, therefore it is possible that the individuals for which this occurred may have reconstructed their views and opinions about STIs so that their reasoning for seeking the test may have changed in light of their diagnosis, however, whatever the reasoning, it is still likely to influence future behaviours (Jutel and Banister, 2013).

\subsection{Conclusion}

This study aimed to illuminate the reasons young people seek STI testing and what factors motivate and influence STI testing behaviour. There were multiple pathways to seeking care which were subject to gender differences. The participants' (both male and female) knowledge about STIs influenced their health-seeking behaviour. Knowledge of the incidence, asymptomatic nature and sequelae of STIs featured prominently in the 
explanations of those who undertook routine testing. Many participants felt that they lacked knowledge about STIs and expressed a desire for more information.

These findings will be important to the design of future public health campaigns aimed at encouraging young people to seek STI testing. These data support the view that primary care physicians should take advantage of any opportunities to educate young people about their STI risk, and also to recommend testing to those under 25 years (NZSHS, 2015a). Males need a particular focus as they are less likely to attend the doctor for other reasons, meaning alternative educational opportunities will need to be exploited, for example continuing sexuality education to the later years of high-school when first sex is likely to occur.

\subsection{Chapter summary and next steps}

This chapter identified five main drivers for STI testing. One of these drivers, STI knowledge, appeared to interact with the others, where better knowledge appeared to encourage more regular STI testing, and poor knowledge was offered as a reason for delayed testing. Given its perceived importance, further investigation of the association between STI knowledge and STI testing behaviour is warranted. This is the focus of the next chapter. 


\section{Chapter 6 STI knowledge and its association with STI testing behaviour}

\subsection{Chapter Overview}

STI knowledge was identified as a driver for testing in Chapter 5. In this next chapter, the association between STI knowledge and STI testing will be investigated in more detail using data from two groups of study participants; SHC attendees and university students.

\subsection{Abstract}

Background: Qualitative data have identified STI knowledge as a potential driver for STI testing. However, most research to date has focussed on STI knowledge in relation to primary prevention (sexual risk behaviour). This study aims to assess STI knowledge among sexually active people in New Zealand, investigate any associated demographic and sexual behaviour characteristics, and determine the association between STI knowledge and previous STI testing. An additional aim was to develop a short STI knowledge assessment tool and compare this to an existing comprehensive tool. Methods: Participants were recruited from a university student health services (SHS) sample and a sexual health clinic (SHC) sample. All participants self-completed a questionnaire which included demographic characteristics, sexual health history and a newly-developed STI knowledge assessment tool. An additional sample of university staff and students was also recruited to test the correlation of the knowledge tool used in this study with a previously validated questionnaire.

Results: The correlation study $(n=200)$ demonstrated that the knowledge tool used in this study was strongly correlated with a previously validated STI knowledge assessment tool, the STD-KQ. The main study ( $\mathrm{n}=582$ people, 346 from SHS and 236 from SHC) demonstrated that knowledge was generally good. Poorly answered questions related to treatment, long-term health consequences and the asymptomatic nature of STIs. Knowledge was associated with alcohol use, smoking, and ethnicity after adjustment for demographic factors, although the association of knowledge with both alcohol use and smoking disappeared after adjustment for sexual behaviour variables. STI knowledge predicted ever having had a test while controlling for demographic and sexual behaviour variables.

Conclusions: STI knowledge is associated with STI testing behaviour. However, the cross-sectional design of this study limits understanding of the direction of causation. As STI knowledge may be improved through educational interventions, this has implications for improving levels of STI testing. 


\subsection{Introduction}

There has been much interest in STI knowledge in relation to STI prevention, which is unsurprising given STI knowledge is a potentially modifiable factor. There has been considerable research into the association of STI knowledge and risky sexual behaviour. For example, cross-sectional studies have shown that a low level of STI knowledge is associated with higher numbers of sexual partners and infrequent condom use (Burazeri et al., 2004, Mmbaga et al., 2008), although the evidence for this association is not conclusive (Norbu et al., 2013, Hoehn et al., 2016).

STI knowledge may also have an impact on whether or not a person presents for STI testing. It is feasible that higher levels of STI knowledge will aid in the identification and interpretation of symptoms as indicators of an STI. Knowing that STIs can be asymptomatic may encourage individuals with no signs or symptoms of infection to seek testing. In addition, knowledge about how STIs are transmitted and their prevalence could impact on a person's STI risk estimation, prompting them to attend for testing. It is possible that having knowledge of the potential long-term health consequences may provide motivation for testing. Lastly, knowledge that many STIs are treatable and curable may encourage a person to attend for testing if they suspect they have been infected.

Despite the rationale, the association between STI knowledge and healthcare-seeking behaviour has not been well researched. The majority of work has been qualitative, where indepth interviews with individuals have uncovered that lack of knowledge is a factor related to delayed or non-existent healthcare-seeking for STI testing (Malta et al., 2007, Chaudhary et al., 2008, Balfe et al., 2012), as reflected in the previous chapter. Building on this, it is important to test if data from a few individuals (qualitative research) can be generalised to a larger sample (quantitative research), and also to determine the magnitude of any association. Only a few studies have attempted to assess the association quantitatively, and these have been restricted to specific population groups such as high-school students (Langille et al., 2009), undergraduate students (Greaves et al., 2009, Cragg et al., 2016), or MSM (Pedrana et al., 2012). These studies have found mixed results. 
To date, there have been no studies assessing STI knowledge in New Zealand, other than those specifically related to HIV or HPV (Chetwynd, 1991, Duncan and Bergen, 1997, Chelimo and Wouldes, 2009, Lachowsky et al., 2014, Henrickson et al., 2015). Therefore, this study aims to assess the level of STI knowledge among sexually active people in New Zealand and identify where knowledge may be lacking (section 6.6.2). This will be achieved by combining data from two different populations, as outlined in section 6.4.1. The association between STI knowledge and various sociodemographic and sexual behaviour characteristics is examined in section 6.6.3, in order to determine which groups of people may benefit most from educational interventions. The association between STI knowledge and STI testing history is investigated in section 6.6.4, the relationship between self-assessed knowledge and measured knowledge is reported in section 6.6.5, and lastly, the influence of STI campaign awareness on STI knowledge and STI testing history is examined in section 6.6.6.

\subsubsection{A note on nomenclature}

Although sometimes used interchangeably in the literature (Dimmitt Champion et al., 2013, Simpson et al., 2015), health-related knowledge is separate and distinct from health literacy. Health literacy is defined as the degree to which individuals have the capacity to obtain, process, and understand basic health information needed to make appropriate health decisions (Healthy People 2010). Knowledge, in this context, can be defined as the range of information an individual holds and understands. Although literacy may be an important predictor of healthcare-seeking behaviour through its moderation on the ability to understand STI information (Fortenberry et al., 2001, Needham et al., 2010), in the interest of time and resources, this analysis focusses only on STI knowledge as an endpoint of this process.

\subsection{Methods}

\subsubsection{Settings and samples}

Overall, three samples were recruited. Two samples were recruited for the main study; a Sexual Health Clinic (SHC) population, and a Student Health Services (SHS) population. 
The SHC, previously described in detail in Chapter 3, is an inner-city public SHC in Wellington, New Zealand. The clinic's services include STI testing, treatment and advice for both self-referred and referred patients, emergency contraception and sexual assault care, as well as accepting referrals from primary and secondary level clinicians for complex sexual health problems. The second sample was recruited from a university SHS, a primary health care service for all students enrolled at a large university in Wellington. It operates full-time clinics at two campus locations, including general practice and counselling services.

A third sample, of university students and staff, was recruited to test the correlation of the knowledge assessment tool used in this study with a previously validated questionnaire.

\subsubsection{Recruitment at the SHC}

Questionnaires were distributed to all new patients at the SHC between September and November 2015 by reception staff for completion before their consultation. The questionnaire was accompanied by a Study Information Sheet which explained the study in detail, including ethical and confidentiality considerations. If a participant declined to participate, this was recorded by the reception staff. All participants were offered the chance to enter a prize draw to win a grocery voucher to thank them for their participation.

\subsubsection{Recruitment at the SHS}

Recruitment at the SHS ran from November 2014 to November 2015 across the two clinic locations. The participants had the option of completing a paper copy and returning to a secure drop-box at the clinic exit, or scanning a Quick Response (QR) code to take them to a secure survey website (Qualtrics software). Questionnaires were initially available in the waiting rooms of the two clinic locations. These recruitment strategies were informed by the results of three focus groups with students which took place before recruitment began (see Appendix B). As participation was self-select, it was not possible to record information about acceptances and refusals. Due to a paperless waiting room policy being implemented in April 2015 at one of the clinic sites, questionnaires were handed out by clinicians for the last few months of recruitment at this site only. All participants were offered the chance to enter a prize draw to win a grocery voucher to thank them for their participation. 


\subsubsection{Recruitment of a sample for the correlation study}

The correlation study sample was drawn from a university population and recruitment took place using a targeted approach. Representation from different groups was sought using email invites and postings on specific social media sites and in newsletters, including Māori and Pacific peoples, people with disabilities, and staff and students from different schools within the University. The study was conducted online only, using secure survey software (Qualtrics software). The survey included a link to a Participant Information Sheet and informed consent was obtained using tick-box options prior to the survey starting. All participants were offered the chance to enter a prize draw to win a grocery voucher to thank them for their participation.

\subsubsection{Development of a knowledge assessment tool and correlation with gold-standard tool}

A review of the literature was conducted to assess which would be the most appropriate STI knowledge assessment tool for use in the current study. A comprehensive search was conducted in PubMed to return articles that assessed STI knowledge. A variety of terms were used including "STI knowledge", "sexual health knowledge" and "sexual health literacy". The resulting articles were screened for relevance and obtained in full if they appeared to assess STI knowledge. Articles were read in full and the relevant data about knowledge assessment was extracted. The corresponding authors of papers were contacted to request a copy of the knowledge tool where the knowledge questions were not presented in the paper but sounded relevant.

Several factors were considered in choosing the knowledge questions for the study:

1. Relevance to the research question

2. Suitability for the population

3. Validity

4. Length

The literature review confirmed that many studies have assessed STI knowledge, mainly in relation to sexual risk behaviour. Although the field is relatively well established, there are very few validated STI assessment knowledge tools available and as a consequence most studies have developed their own measure of STI knowledge (Foster and Byers, 2008). 
As the knowledge assessment needed to be incorporated into a paper questionnaire and be completed in the time between arrival at the clinic and being called in for consultation, many of the existing comprehensive tools were unsuitable due to their length (ARCSHS Secondary Students and Sexual Health Survey (Smith et al., 2009), Sexually Transmitted DiseasesKnowledge Questionnaire (Jaworski and Carey, 2007), Sex Knowledge, Experience, and Needs scale (McCabe et al., 1999), University of Missouri Sexual Health Survey (EastmanMueller et al., 2010)). Therefore, a shorter tool was developed from the results of the literature review and a correlation study of this with a validated questionnaire was designed.

Development of the short tool involved selecting questions to cover a variety of aspects of STI knowledge including transmission, symptoms, prevention, complications, treatment and different types of infection. The questions were selected from two main sources, the National Survey of Adolescents and Young Adults conducted in the USA (National Survey of Adolescents and Young Adults: Sexual Health Knowledge, Attitudes and Experiences, 2003), and a study by Malacova et al. (2011) assessing STI knowledge in prisoners and the general population in Australia. Questions were trialled on students who were then asked for their feedback. From this, a seven-item true-false questionnaire was developed:

1. 'Chlamydia only affects women'

2. 'Chlamydia can lead to infertility in women'

3. 'Once a person has caught genital herpes, they will always have the virus'

4. 'STIs can only be spread when symptoms are present'

5. 'Gonorrhoea can usually be treated with antibiotics'

6. 'Many STIs will cause only mild or no symptoms'

7. 'Some kinds of cancer can be caused by STIs'

The seven questions each consisted of a statement to which participants were asked to identify whether it was 'True' or 'False'. A further option of 'I don't know' was included and participants were encouraged to select this option if they did not know whether the statement was true or false, rather than guess the answer. When a knowledge question was missed out by a participant it was recorded as missing data rather than as not knowing the answer because the participant had not selected the option 'I don't know'. Every correct answer scored one point, and every wrong answer and 'I don't know' answer scored zero points. The scores were then summed to give a total knowledge score from a total of seven for each participant. 
In addition to this core set of questions, some additional items were developed, including the first question in the section which asked the participant to self-assess their level of knowledge so that a comparison could be made with their actual level of knowledge. Focus group discussions (see Appendix B) and anecdotal conversations with students also informed a population-specific question to come after the core questions: 'Are you aware of any STI campaigns in New Zealand, either now or in the past?'

The literature review revealed that an established tool, named the Sexually Transmitted Diseases - Knowledge Questionnaire (STD-KQ) (Jaworski and Carey, 2007), had excellent reliability scores and had considerable relevance to the current research project and the population of interest. Therefore it was selected as the gold-standard tool with which to compare the newly developed seven-item assessment tool in the correlation study.

\subsubsection{Questionnaire content}

The questionnaire included questions about participants' basic demographic characteristics, sexual behaviour, STI testing history, as well as the seven-item STI knowledge assessment tool. More information about the variables used in the questionnaire can be found in Appendix A.

\subsubsection{Data analysis}

\subsubsection{Exclusion criteria}

The SHS sample included both testers and non-testers, with $14.9 \%$ of the latter group answering 'no', 'not sure' or 'would prefer not to say' to a question about having ever been sexually active. Initial analyses among the SHS sample demonstrated that people who had ever been sexually active had significantly higher knowledge scores than people who had never been sexually active (data not shown). Therefore, people who had never been sexually active were excluded from further analyses on the basis that they would have little need for STI testing and their scores would have biased results. As stated earlier, the aim of this study was to assess the level of STI knowledge among sexually active people in New Zealand. Those who had selected 'not sure' $(n=2)$ or 'would prefer not to say' $(n=6)$ were also excluded as it was not clear which group they fell into. 


\subsubsection{Statistical methods}

Chi-squared tests were used to compare samples for categorical variables including demographic characteristics, testing history and sexual behaviour. Fisher's exact tests were used when expected cell counts were less than five. Binomial logistic regression was used to assess differences between groups when looking at a binary response, for example when determining if there was a difference in knowledge for individual questions between samples. Ordinal logistic regression was used when modelling the association with total knowledge score as an ordinal scale variable. Mann-Whitney U tests (and Kruskal-Wallis tests for variables with more than two levels) were performed to assess whether there were differences in continuous variables between two groups. Prior to each analyses, assumptions were assessed, including checking for outliers and assessment of linear relationships in the case of continuous predictor variables using the Box-Tidwell (1962) procedure. All statistical analyses were performed using SPSS (version 23), and all tests were carried out using a 0.05 level of significance.

\subsubsection{Ethical approval}

Ethical approval was granted by Victoria University of Wellington Human Ethics Committee (ref: 20504) (Appendix D).

\subsection{Results of the correlation study}

In total, 258 people started the online questionnaire, which included both the seven-item knowledge assessment tool and the 27-item gold-standard tool. Of these, 226 completed all of the knowledge questions. A sexually active sample was selected for analysis as this is the population of interest ( $n=200,31 \%$ male, $69 \%$ female). A Spearman's rank-order correlation was run to assess the relationship between the scores for the full STD-KQ (from a total of 27) and the scores for the seven-item brief knowledge questionnaire for the online sample. Preliminary analysis showed the relationship to be monotonic, as assessed by visual inspection of a scatterplot. There was a strong positive correlation between the two questionnaires, $r_{\mathrm{s}}(198)=0.721, p<0.001$, i.e. as STD-KQ scores increased, so did the sevenitem score, as shown in Table 6.1. This suggests that the seven-item knowledge questionnaire 
would distinguish between those with higher knowledge and those with lower knowledge in a similar manner to the validated STD-KQ.

Table 6.1. Mean, median, minimum and maximum values for the STD-K questionnaire for each scoring level of the 7-item knowledge questionnaire

\begin{tabular}{cccccc}
\hline & \multicolumn{5}{c}{ STD-K questionnaire } \\
\cline { 2 - 6 } Total score for 7-item questionnaire & $\boldsymbol{n}$ & $\begin{array}{c}\text { Mean } \\
\text { (SD) }\end{array}$ & Median & Minimum & Maximum \\
\hline 0 & 7 & 2 & 0 & 0 & 11 \\
1 & 9 & 2 & 1 & 0 & 12 \\
2 & 15 & 5 & 5 & 0 & 12 \\
3 & 19 & 9 & 9 & 2 & 17 \\
4 & 35 & 13 & 14 & 3 & 21 \\
5 & 35 & 13 & 15 & 3 & 21 \\
6 & 42 & 16 & 17 & 3 & 25 \\
7 & 49 & 20 & 21 & 6 & 27 \\
\hline
\end{tabular}

\subsection{Results of the main study}

\subsubsection{Characteristics of the samples}

The knowledge questionnaire was completed by 346 sexually active individuals at the SHS and 236 individuals at the SHC. The recruitment rate for the SHC was $73.4 \%$ as detailed in Chapter 3. A recruitment rate could not be calculated for the SHS group for reasons discussed in section 6.4.3. The demographic characteristics of the SHC study sample were compared to the characteristics of all clients attending the clinic during the study period using anonymised raw data from the SHC. Pearson's chi-squared tests revealed no significant differences between the study sample and the whole clinic population for gender or ethnicity, however the study sample was slightly younger $(43.8 \%<25$ yrs vs $26 \%<25$ yrs $)$. Raw data on the general SHS population were not available, but overall statistics were compared with the study sample which revealed a similar structure for age $(81.7 \%<25 \mathrm{yrs}$ in study sample vs $80.7 \% \leq 26 y r s$ in SHS population) and ethnicity (76.5\% vs 75\% NZ European/Pākehā, 9.7\% vs 5\% Māori, $0.9 \%$ vs 3\% Pacific peoples, $5.0 \%$ vs $7 \%$ Asian, $7.9 \%$ vs $10 \%$ Other), but a difference in gender (67\% female in study sample vs $57 \%$ female in SHS population). 
Tables 6.2 and 6.3 present the demographic characteristics, testing history and sexual behaviours by sample. Chi-square tests were used to assess the association between study sample (SHS or SHC) and the various demographic, sexual behaviour and testing characteristics. Where expected cell frequencies were less than five, Fisher's exact test (FET) was used instead. There was a statistically significant association between sample and gender, with a greater proportion of women in the SHS sample $(67.2 \%)$ than the SHC sample $(47.9 \%)\left(p<0.001\right.$, FET). There was also an association between sample and age, $X^{2}(2)=$ 88.718, $p<0.001$, with the SHS sample being younger. Ethnicity also differed $(p<0.001$, FET), with the SHC having a higher proportion of participants selecting 'Other' as their ethnicity than the SHS sample. Smoking differed between the two samples with the SHC having a higher proportion of smokers than SHS $(24.6 \%$ vs $14.8 \%), X^{2}(1)=8.720, p=0.003$. The SHC also had a slightly larger proportion of heavy drinkers as assessed by the AUDIT-C questionnaire (60.6\% vs 52.8\%), although this difference was not statistically significant, $X^{2}(1)=3.472, p=0.062$.

Participants reported similar condom use and relationship status between the two samples. As there was a statistically significant difference between the samples for gender, the association between sample and sexual orientation behaviour was analysed for males and females separately as binary variables, rather than a four level grouped variable. There was no association between sample and sexual orientation behaviour for men $\left(X^{2}(1)=0.069, p=\right.$ 0.793 ) or women $\left(X^{2}(1)=1.260, p=0.262\right)$. A Mann-Whitney $\mathrm{U}$ test was run to determine if there were differences in the total number of sexual partners over the past 12 months between those attending SHS and those attending the SHC. Distributions of the number of partners for each sample were similar, as assessed by visual inspection of box-plots. The median number of partners was statistically significantly higher in the SHC group (median $=3$ ) than in the SHS group (median $=2), U=10713.5, Z=-3.457, p=0.001$.

Previous testing behaviour for STI was similar between the samples, however there was a statistically significant difference in previous HIV testing between the two groups, with a higher proportion of the SHC group ever having had a HIV test compared with the SHS group $(50.7 \%$ vs $22.8 \%), X^{2}(1)=23.837, p<0.001$. 
In conclusion, the results of these analyses in this section suggest that the two samples, SHC and SHS, are different in terms of their demographic characteristics, but similar in their sexual behaviour with the exception of the SHC group having a higher number of sexual partners in the past 12 months.

Table 6.2. Comparison of demographic characteristics and testing history of those attending Student Health Services and those attending the Sexual Health Clinic

\begin{tabular}{|c|c|c|c|c|}
\hline \multirow[t]{2}{*}{ Characteristic } & \multicolumn{2}{|c|}{$\%(n)^{\mathrm{a}}$} & \multirow{2}{*}{$\begin{array}{l}\text { Chi-squared } \\
\text { statistic }(d f)\end{array}$} & \multirow[t]{2}{*}{$p$ value } \\
\hline & $\begin{array}{c}\text { SHS } \\
N=346\end{array}$ & $\begin{array}{c}\text { SHC } \\
N=236\end{array}$ & & \\
\hline Gender & & & FET & $<0.001$ \\
\hline Male & $30.5(105)$ & $52.1(123)$ & & \\
\hline Female & $67.2(231)$ & $47.9(113)$ & & \\
\hline Other & $2.3(8)$ & $0.0(0)$ & & \\
\hline Age & & & $88.718(2)$ & $<0.001$ \\
\hline$<25$ & $81.7(272)$ & $43.5(101)$ & & \\
\hline $25-34$ & $13.2(44)$ & $40.1(93)$ & & \\
\hline $35+$ & $5.1(17)$ & $16.4(38)$ & & \\
\hline Ethnicity & & & FET & $<0.001$ \\
\hline Māori & $9.7(33)$ & $6.9(16)$ & & \\
\hline Pacific peoples & $0.9(3)$ & $0.9(2)$ & & \\
\hline Asian & $5.0(17)$ & $5.6(13)$ & & \\
\hline Other & $7.9(27)$ & $40.3(94)$ & & \\
\hline New Zealand European & $76.5(261)$ & $46.4(108)$ & & \\
\hline Alcohol use & & & $3.472(1)$ & 0.062 \\
\hline AUDIT-C score $<5$ & $47.2(162)$ & $39.4(93)$ & & \\
\hline AUDIT-C score $\geq 5$ & $52.8(181)$ & $60.6(143)$ & & \\
\hline Smoking & & & $8.720(1)$ & 0.003 \\
\hline Yes & $14.8(51)$ & $24.6(58)$ & & \\
\hline No & $85.2(293)$ & $75.4(178)$ & & \\
\hline Ever had an STI test in the past & & & $1.848(1)$ & 0.202 \\
\hline Yes & $70.3(90)$ & $63.2(146)$ & & \\
\hline No & $29.7(38)$ & $36.8(85)$ & & \\
\hline Ever had a HIV test in the past & & & $23.837(1)$ & $<0.001$ \\
\hline Yes & $22.8(26)$ & $50.7(107)$ & & \\
\hline No & $77.2(88)$ & $49.3(104)$ & & \\
\hline
\end{tabular}


Table 6.3. Comparison of sexual behaviours of those attending Student Health Services and those attending the Sexual Health Clinic

\begin{tabular}{|c|c|c|c|c|}
\hline \multirow[t]{2}{*}{ Characteristic } & \multicolumn{2}{|c|}{$\%(n)^{\mathrm{a}}$} & \multirow{2}{*}{$\begin{array}{l}\text { Chi-squared } \\
\text { statistic }(d f)\end{array}$} & \multirow[t]{2}{*}{$p$ value } \\
\hline & $\begin{array}{c}\text { SHS }^{\mathbf{b}} \\
N=\mathbf{1 3 0}\end{array}$ & $\begin{array}{c}\text { SHC } \\
N=236\end{array}$ & & \\
\hline \multicolumn{3}{|l|}{ Relationship status } & $1.222(1)$ & \multirow[t]{3}{*}{0.269} \\
\hline In a relationship & $49.2(64)$ & $43.2(102)$ & & \\
\hline Single & $50.8(66)$ & $56.8(134)$ & & \\
\hline \multicolumn{3}{|l|}{ Condom use } & $2.556(1)$ & \multirow[t]{3}{*}{0.110} \\
\hline Frequent & $38.8(50)$ & $47.5(112)$ & & \\
\hline Infrequent & $61.2(79)$ & $52.5(124)$ & & \\
\hline \multicolumn{3}{|l|}{ Sexual orientation behaviour in men } & $0.069(1)$ & \multirow{3}{*}{0.793} \\
\hline Non-MSM & $74.1(20)$ & $71.6(83)$ & & \\
\hline MSM & $25.9(7)$ & $28.4(33)$ & & \\
\hline \multicolumn{3}{|l|}{ Sexual orientation behaviour in women } & $1.260(1)$ & \multirow[t]{3}{*}{0.262} \\
\hline Non-WSW & $95.7(88)$ & $91.7(100)$ & & \\
\hline \multirow[t]{2}{*}{ WSW } & $4.3(4)$ & $8.3(9)$ & & \\
\hline & $\begin{array}{c}\text { Median } \\
(n)\end{array}$ & $\begin{array}{c}\text { Median } \\
(n)\end{array}$ & $\boldsymbol{U}$ & $p$ value \\
\hline Number of sexual partners & $2(125)$ & $3(220)$ & 10713.5 & 0.001 \\
\hline \multicolumn{5}{|c|}{ 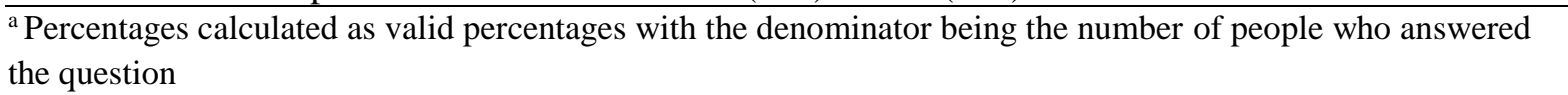 } \\
\hline \multirow{2}{*}{\multicolumn{5}{|c|}{$\begin{array}{l}\text { b Only those attending for a sexual health related appointment (STI test, contraception, cervical smear test) were } \\
\text { asked this set of questions, meaning the denominator is smaller than for demographic variables } \\
\text { MSM = men who have sex with men }\end{array}$}} \\
\hline & & & & \\
\hline WSW = women who have sex with womer & & & & \\
\hline
\end{tabular}

\subsubsection{STI knowledge in SHS and SHC}

The proportion of people answering each question correctly is detailed in Table 6.4.

Knowledge was generally good, with the majority of people getting the correct answer for many of the questions. The questions that were answered poorly were: 'Gonorrhoea can usually be treated with antibiotics', which $60.2 \%$ of SHS participants and $50.4 \%$ of SHC participants answered incorrectly, and 'Some kinds of cancer can be caused by STIs' which $50.0 \%$ of SHS participants and $45.5 \%$ of SHC participants answered incorrectly. In addition, a third of people (32.8\% of SHS participants and 34.0\% of SHC participants) did not know that many STIs caused only mild or no symptoms.

A total score out of seven was computed for each individual. Within the SHC sample, one person missed one question out of seven and one person missed two questions. In the SHS 
sample, three people missed one question, one missed four questions and one person missed six questions out of seven. A total knowledge score was not calculated for these people, so in total there were seven people without a total knowledge score. Overall, the mean score was $4.85(\mathrm{SD}=1.657)$ out of 7 , with the distribution negatively skewed (Figure 6.1).

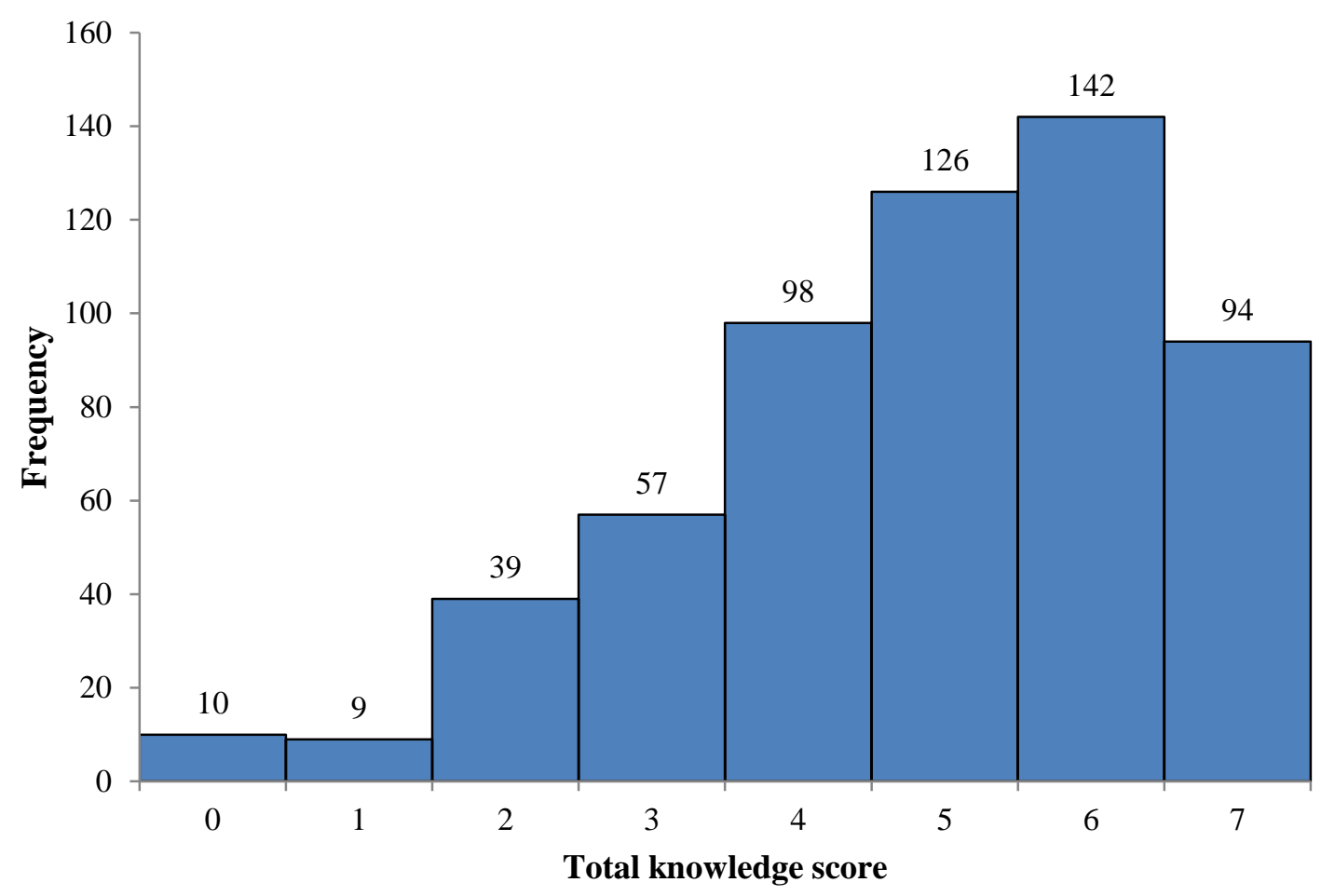

Figure 6.1. Histogram showing frequencies of total knowledge scores for both samples combined (SHS and SHC)

Binomial logistic regression was used to assess the association between study sample and selecting the correct answer for each individual knowledge question (Table 6.4). There were no statistically significant associations between the sample and any of the knowledge questions, other than the question 'Gonorrhoea can usually be treated with antibiotics' $\left(X^{2}(1)\right.$ $=5.407, p=0.020$ ) which was answered correctly by a slightly larger proportion of the SHC sample than the SHS sample (49.6\% vs $39.8 \%)$. 
Ordinal logistic regression was used to assess whether there were differences in the total knowledge score between the two samples. No difference in total score between the samples was observed $(\mathrm{OR}=0.844,95 \%$ CI $0.629-1.133, p=0.259)$.

Due to there being some differences in demographics between the two samples (Tables 6.2 and 6.3), the association between knowledge and sample was also analysed using binomial logistic regression with adjustment for demographic variables: controlling for age, gender, ethnicity, smoking and alcohol. None of the odds ratios were statistically significant in the models that included these variables, indicating there were no differences in the scores for the individual knowledge questions between the two groups (Table 6.4). Ordinal logistic regression was used to assess differences in total knowledge score between samples with adjustment for the demographic variables. The odds ratio resulting from this model was also not statistically significant $(\mathrm{OR}=1.135,95 \%$ CI $0.790-1.629, p=0.494)$ (Table 6.4). Expanded binomial logistic and ordinal regression models were also conducted including the sexual behaviour variables: relationship status (single, in a relationship), condom use (frequent, infrequent), sexual orientation behaviour (non-MSM, MSM, non-WSW, WSW) and number of sexual partners. The addition of these variables did not change the outcome of the analyses; there was still no relationship between knowledge and sample for any of the knowledge questions or the total knowledge score (data not shown).

In conclusion, there were no differences in STI knowledge between the two samples, despite differences in demographic characteristics seen in section 6.6.1. In addition, the analyses described in this section suggest that although STI knowledge is generally good, there are some areas in which knowledge is lacking. 


\section{Table 6.4. Comparison of correct response to individual knowledge questions between SHS and SHC samples}

\begin{tabular}{|c|c|c|c|c|c|c|c|c|c|c|}
\hline \multirow[b]{3}{*}{ Knowledge question } & \multirow{2}{*}{\multicolumn{2}{|c|}{$\%(n)$ correct $^{\mathrm{a}}$}} & \multicolumn{4}{|c|}{ Unadjusted analyses } & \multicolumn{4}{|c|}{ Adjusted analyses ${ }^{\mathrm{c}}$} \\
\hline & & & \multirow[t]{2}{*}{$n$} & \multirow[t]{2}{*}{$\mathbf{O R}^{\mathbf{b}}$} & \multirow[t]{2}{*}{$95 \% \mathrm{CI}$} & \multirow[t]{2}{*}{$p$ value } & \multirow[t]{2}{*}{$n$} & \multirow[t]{2}{*}{$\mathbf{O} \mathbf{R}^{\mathbf{b}}$} & \multirow[t]{2}{*}{$95 \% \mathrm{CI}$} & \multirow[t]{2}{*}{$p$ value } \\
\hline & $\begin{array}{r}\text { SHS } \\
\mathrm{N}=346\end{array}$ & $\begin{array}{c}\text { SHC } \\
\mathbf{N}=\mathbf{2 3 6}\end{array}$ & & & & & & & & \\
\hline 'Chlamydia only affects women' & & & 580 & 1.106 & $0.695-1.762$ & 0.670 & 543 & 1.206 & $0.663-2.192$ & 0.540 \\
\hline Correct & $84.3(290)$ & $85.6(202)$ & & & & & & & & \\
\hline Incorrect/didn't know & $15.7(54)$ & $14.4(34)$ & & & & & & & & \\
\hline 'Chlamydia can lead to infertility in women' & & & 578 & 1.061 & $0.722-1.559$ & 0.762 & 542 & 0.862 & $0.531-1.401$ & 0.549 \\
\hline Correct & $74.6(256)$ & $75.7(178)$ & & & & & & & & \\
\hline Incorrect/didn't know & $25.4(87)$ & $24.3(57)$ & & & & & & & & \\
\hline $\begin{array}{l}\text { 'Once a person has caught genital herpes, they will } \\
\text { always have the virus' }\end{array}$ & & & 581 & 1.218 & $0.835-1.776$ & 0.307 & 544 & 1.024 & $0.642-1.634$ & 0.919 \\
\hline Correct & $71.6(247)$ & $75.4(178)$ & & & & & & & & \\
\hline Incorrect/didn't know & $28.4(98)$ & $24.6(58)$ & & & & & & & & \\
\hline 'STIs can only be spread when symptoms are present' & & & 581 & 0.656 & $0.378-1.140$ & 0.135 & 544 & 0.556 & $0.274-1.127$ & 0.103 \\
\hline Correct & $91.9(317)$ & $88.1(208)$ & & & & & & & & \\
\hline Incorrect/didn't know & $8.1(28)$ & $11.9(28)$ & & & & & & & & \\
\hline 'Gonorrhoea can usually be treated with antibiotics' & & & 580 & 1.486 & $1.063-2.075$ & 0.020 & 543 & 1.008 & $0.665-1.528$ & 0.970 \\
\hline Correct & $39.8(137)$ & $49.6(117)$ & & & & & & & & \\
\hline Incorrect/didn't know & $60.2(207)$ & $50.4(119)$ & & & & & & & & \\
\hline 'Many STIs will cause only mild or no symptoms' & & & 579 & 0.948 & $0.667-1.347$ & 0.765 & 542 & 0.846 & $0.546-1.312$ & 0.456 \\
\hline Correct & $67.2(231)$ & $66.0(155)$ & & & & & & & & \\
\hline Incorrect/didn't know & $32.8(113)$ & $34.0(80)$ & & & & & & & & \\
\hline 'Some kinds of cancer can be caused by STIs' & & & 579 & 1.196 & $0.858-1.668$ & 0.291 & 542 & 1.066 & $0.708-1.607$ & 0.759 \\
\hline Correct & $50.0(172)$ & $54.5(128)$ & & & & & & & & \\
\hline Incorrect/didn't know & $50.0(172)$ & $45.5(107)$ & & & & & & & & \\
\hline & Med & lian $(n)$ & $n$ & $\mathbf{O R}^{\mathbf{b}}$ & $95 \% \mathrm{CI}$ & $p$ value & $n$ & $\mathbf{O R}^{\mathrm{b}, \mathrm{d}}$ & $95 \% \mathrm{CI}$ & $p$ value \\
\hline Total score & $5(341)$ & $5(234)$ & 575 & 0.844 & $0.629-1.133$ & 0.259 & 548 & 1.135 & $0.790-1.629$ & 0.494 \\
\hline
\end{tabular}




\subsubsection{Differences in STI knowledge by sociodemographic and risk behaviour characteristics}

To assess whether there were differences in total knowledge score between various demographic factors and sexual behaviours, Mann-Whitney U tests and Kruskal-Wallis tests were performed for the entire sample (Table 6.5). A Spearman's correlation was also carried out to assess whether knowledge was related to number of sexual partners in the past year.

The results of these analyses revealed that there was a significant difference in total knowledge score between heavy alcohol users and non-heavy alcohol users as assessed by AUDIT-C, where heavy alcohol users had better knowledge $(U=34107.5, Z=-3.191, p<$ 0.001). There was also a difference in total knowledge score between smokers and nonsmokers (smokers had better knowledge) $(U=20615.5, Z=-3.060, p=0.002)$, and between those who were single and those who were in a relationship (those who were single had better knowledge) $(U=13688.0, Z=-2.802, p=0.005)$. There were no significant differences in total knowledge score for gender, age, ethnicity, sexual orientation behaviour, condom use or previous STI diagnosis. Lastly, there was a statistically significant weak positive correlation between total knowledge score and number of sexual partners in the past year, $r_{\mathrm{s}}(342)=0.170, p=0.002$, meaning the knowledge score tended to increase as the number of sexual partners increased. The analyses were repeated for gender without the inclusion of the 'Other' group and for ethnicity without the inclusion of the Pacific peoples group due to small numbers, with no significant changes to the statistical results (data not shown).

To assess whether these results were consistent across samples, the analyses were repeated for each sample separately (Tables AC.1 and AC.2 of Appendix C). The same differences were seen for the SHS sample as had been observed in the combined analyses, but the differences in knowledge by alcohol, smoking and relationship status were no longer present for the SHC sample, indicating that the SHS sample were driving the differences seen in the combined analysis. A significant difference in knowledge score was seen between ethnic groups within the SHC sample when analysed separately, with Māori having the highest scores, followed by the Other group, New Zealand European, Asian and then Pacific peoples $(H(4)=10.611, p=0.031)$. This difference was still statistically significant after removing the 
Pacific peoples group due to small numbers. Number of sexual partners correlated positively with total knowledge score for both samples when analysed separately $\left(r_{\mathrm{s}}(123)=0.215, p=\right.$ 0.016 for SHS and $r_{\mathrm{s}}(217)=0.133, p=0.049$ for SHC), meaning that for both groups the knowledge score tended to increase as the number of sexual partners increased.

Table 6.5. Difference in knowledge by sociodemographic and sexual behaviour characteristics

\begin{tabular}{|c|c|c|c|c|c|}
\hline Characteristic & $n$ & Mean rank & $\boldsymbol{U}$ & $Z$ & $p$ value \\
\hline Alcohol use & & & 34107.500 & -3.191 & 0.001 \\
\hline AUDIT-C score $<5$ & 250 & 261.93 & & & \\
\hline AUDIT-C score $\geq 5$ & 322 & 305.58 & & & \\
\hline Smoking & & & 20615.500 & -3.060 & 0.002 \\
\hline Yes & 109 & 329.87 & & & \\
\hline No & 464 & 276.93 & & & \\
\hline Relationship status & & & 13688.000 & -2.802 & 0.005 \\
\hline Single & 198 & 196.37 & & & \\
\hline In a relationship & 166 & 165.96 & & & \\
\hline Condom use & & & 14920.000 & -1.357 & 0.175 \\
\hline Frequent & 160 & 190.25 & & & \\
\hline Infrequent & 203 & 175.50 & & & \\
\hline Sexual orientation behaviour in men & & & 1661.000 & -1.622 & 0.105 \\
\hline Non-MSM & 103 & 68.13 & & & \\
\hline MSM & 39 & 80.41 & & & \\
\hline Sexual orientation behaviour in women & & & 965.500 & -1.264 & 0.206 \\
\hline Non-WSW & 187 & 99.16 & & & \\
\hline \multirow[t]{2}{*}{ WSW } & 13 & 119.73 & & & \\
\hline & $n$ & Mean rank & $H(d f)$ & & $p$ value \\
\hline Gender & & & $0.535(2)$ & & 0.765 \\
\hline Male & 224 & 288.02 & & & \\
\hline Female & 341 & 285.39 & & & \\
\hline Other & 8 & 327.31 & & & \\
\hline Age & & & $3.036(2)$ & & 0.219 \\
\hline$<25$ & 369 & 271.68 & & & \\
\hline $25-34$ & 135 & 297.76 & & & \\
\hline $35+$ & 55 & 292.24 & & & \\
\hline Ethnicity & & & $6.809(4)$ & & 0.146 \\
\hline Māori & 49 & 292.97 & & & \\
\hline Pacific peoples & 5 & 278.10 & & & \\
\hline Asian & 29 & 219.21 & & & \\
\hline Other & 121 & 303.88 & & & \\
\hline \multirow[t]{2}{*}{ New Zealand European } & 363 & 281.42 & & & \\
\hline & & $\begin{array}{l}\text { Spearman's } \\
\text { correlation }\end{array}$ & & & $p$ value \\
\hline Number of sexual partners & 344 & 0.170 & & & 0.002 \\
\hline
\end{tabular}


To adjust for covariates, ordinal logistic regression analyses were carried out for the two samples combined (Table 6.6) and separately (Tables AC.3 and AC.4 of Appendix C). The variables included in the model were age, gender, ethnicity, smoking and alcohol. The adjusted regression analyses showed similar results to that seen in Table 6.5 of this chapter and AC.1 and AC.2 of Appendix C. Ethnicity, alcohol and smoking predicted knowledge, with SHS driving the effect of alcohol and smoking on knowledge and SHC driving the effect of ethnicity on knowledge. Those who had AUDIT-C scores less than 5 (lower level of alcohol use) had, on average, lower knowledge scores than those who had AUDIT-C scores equal to or more than $5(\mathrm{OR}=0.677,95 \% \mathrm{CI} 0.491-0.935, p=0.018)$. People that did not smoke had, on average, lower knowledge scores than those who did smoke $(\mathrm{OR}=0.651$, 95\% CI $0.440-0.961, p=0.031)$. People who identified as Asian had, on average, lower knowledge scores than people who identified as New Zealand European ( $\mathrm{OR}=0.434,95 \%$ CI $0.219-0.859, p=0.017$ ). These analyses were repeated without the Other group in gender and without the Pacific peoples group in ethnicity, due to small numbers in these groups. No differences in the models were observed when these groups were removed (data not shown).

Sexual behaviour characteristics (relationship status, condom use and total number of partners in past year) were also added into the model. Sexual orientation behaviour was not included as this variable is split by gender meaning the two variables are measuring very similar information. None of the sexual behaviour variables were independently associated with STI knowledge (all $p>0.05$ ) (Table AC.5 of Appendix C). After adding in the sexual behaviour variables to the model, only Asian ethnicity remained a significant predictor of poorer knowledge (Table AC.5 of Appendix C). Alcohol use and smoking no longer predicted knowledge. This is likely due to alcohol and smoking being covariates of sexual behaviour. Alcohol use was statistically significantly associated with risky sexual behaviour, where those who had an AUDIT-C score more than or equal to 5 had a higher number of sexual partners (median $=4)$ compared with those with a score less than 5 (median $=2), U=$ $8535, p<0.001$. Smoking was also related to risky sexual behaviour, where those who 
smoked had a higher number of sexual partners (median $=4)$ than non-smokers $($ median $=3)$, $U=6657.5, p<0.001$. Neither alcohol use nor smoking were related to condom use.

In conclusion, lower alcohol use as assessed by AUDIT-C score, not smoking, and Asian ethnicity, independently predicted having a lower STI knowledge score prior to adjustment for sexual behaviour. After adjustment for sexual behaviour characteristics, only Asian ethnicity predicted poorer knowledge.

Table 6.6. The effect of sociodemographic variables on total knowledge score for the whole sample $(n=546)$

\begin{tabular}{lccc}
\hline Characteristic & OR & 95\% CI & $p$ value \\
\hline Gender & & & 0.534 \\
$\quad$ Male & 0.914 & $0.666-1.256$ & 0.580 \\
$\quad$ Other & 1.887 & $0.490-7.262$ & 0.356 \\
$\quad$ Female & 1 & & \\
Age & 0.677 & $0.395-1.159$ & 0.184 \\
$\quad<25$ & 0.899 & $0.501-1.610$ & 0.719 \\
$25-34$ & 1 & & \\
35+ & 1.026 & $0.592-1.779$ & 0.926 \\
Ethnicity & 0.912 & $0.192-4.337$ & 0.908 \\
$\quad$ Māori & 0.434 & $0.219-0.859$ & 0.017 \\
$\quad$ Pacific peoples & 1.171 & $0.792-1.732$ & 0.429 \\
$\quad$ Asian & 1 & & \\
$\quad$ Other & & & \\
$\quad$ New Zealand European & 0.677 & $0.491-0.935$ & 0.018 \\
Alcohol use & 1 & & \\
$\quad$ AUDIT-C score <5 & & & \\
$\quad$ AUDIT-C score $\geq 5$ & 0.651 & $0.440-0.961$ & 0.031 \\
Smoking & 1 & & \\
$\quad$ No & & & \\
$\quad$ Yes &
\end{tabular}

\subsubsection{Difference in knowledge by testing history in SHS and SHC}

The qualitative data presented in Chapter 5 suggested that knowing more about STIs may influence testing behaviour. This relationship can be explored using the quantitative data from the study presented in this chapter. An assessment of the difference in level of knowledge between those who had had an STI test before and those who were attending for the first time (not had a test before) was carried out using a Mann-Whitney U analysis. The 
individuals who had tested before had significantly better knowledge than those who were attending for the first time $(U=10089.500, Z=-4.684, p<0.001)$ (Table 6.7). A similar result was found when the analysis was restricted to just those who had had a HIV test before $(U=9899.0, Z=-3.355, p=0.001)$ (Table 6.7).

Table 6.7. Difference in knowledge by testing history

\begin{tabular}{|c|c|c|c|c|c|c|}
\hline & \multicolumn{6}{|c|}{ Combined } \\
\hline & $n$ & Median & Mean rank & $U$ & $Z$ & $p$ value \\
\hline Ever had an STI test & & & & 10089.500 & -4.684 & $<0.001$ \\
\hline No & 122 & 5 & 144.20 & & & \\
\hline Yes & 235 & 5 & 197.20 & & & \\
\hline Ever had a HIV test & & & & 9899.000 & -3.355 & 0.001 \\
\hline No & 191 & 5 & 147.83 & & & \\
\hline \multirow[t]{3}{*}{ Yes } & 132 & 6 & 182.51 & & & \\
\hline & \multicolumn{6}{|c|}{ Student Health Services } \\
\hline & $n$ & Median & Mean rank & $\boldsymbol{U}$ & $Z$ & $p$ value \\
\hline Ever tested & & & & 1191.500 & -2.761 & 0.006 \\
\hline No & 38 & 4 & 50.86 & & & \\
\hline Yes & 90 & 5 & 70.26 & & & \\
\hline Ever had a HIV test & & & & 777.500 & -2.535 & 0.011 \\
\hline No & 88 & 5 & 53.34 & & & \\
\hline \multirow[t]{3}{*}{ Yes } & 26 & 6 & 71.60 & & & \\
\hline & \multicolumn{6}{|c|}{ Sexual Health Clinic } \\
\hline & $n$ & Median & Mean rank & $U$ & $Z$ & $p$ value \\
\hline Ever tested & & & & 4222.500 & -3.946 & $<0.001$ \\
\hline No & 84 & 5 & 92.77 & & & \\
\hline Yes & 145 & 6 & 127.88 & & & \\
\hline Ever had a HIV test & & & & 4566.500 & -2.090 & 0.037 \\
\hline No & 103 & 5 & 96.33 & & & \\
\hline Yes & 106 & 5.5 & 113.42 & & & \\
\hline
\end{tabular}

This relationship was further tested by assessing the effect of knowledge on testing behaviour using binomial logistic regression with adjustment for age, gender, ethnicity, smoking, alcohol, relationship status, condom use and total number of partners in past year (Table 6.8). The logistic regression model was statistically significant, $X^{2}(13)=44.804, p<0.001$. Of the eight predictor variables, only three were statistically significant: STI knowledge, gender, and total number of partners in past 12 months (Table 6.8). For each additional STI 
knowledge question an individual answered correctly, on average they were 1.4 (1.217 1.694) times more likely to have had an STI test in the past. Females had $2.9(1.627-5.020)$ times higher odds of having tested than males. Increasing number of partners was also independently associated with an increased likelihood of having previously tested, although the OR was small at just $1.1(1.009-1.152)$. A similar relationship was seen when predicting ever having had a HIV test, although gender was no longer predictive of testing but older age and the ethnic group 'Other' were (data not shown).

Table 6.8. Predictors of having had a previous STI test $(n=322)$

\begin{tabular}{|c|c|c|c|c|c|c|c|}
\hline & $\boldsymbol{B}$ & S.E. & Wald & $d f$ & $p$ value & OR & $95 \% \mathrm{CI}$ \\
\hline Total knowledge score & 0.362 & 0.084 & 18.39 & 1 & $<0.001$ & 1.436 & $1.217-1.694$ \\
\hline Gender & 1.05 & 0.287 & 13.348 & 1 & $<0.001$ & 2.858 & $1.627-5.020$ \\
\hline Age & & & 2.435 & 2 & 0.296 & & \\
\hline $25-34$ years & 0.489 & 0.326 & 2.248 & 1 & 0.134 & 1.631 & $0.860-3.094$ \\
\hline $35+$ years & 0.01 & 0.454 & 0.000 & 1 & 0.982 & 1.01 & $0.415-2.457$ \\
\hline Ethnicity & & & 1.696 & 4 & 0.792 & & \\
\hline Māori & -0.258 & 0.533 & 0.235 & 1 & 0.628 & 0.772 & $0.272-2.194$ \\
\hline Pacific peoples & 0.482 & 1.317 & 0.134 & 1 & 0.714 & 1.619 & $0.123-21.389$ \\
\hline Asian & 0.129 & 0.616 & 0.044 & 1 & 0.835 & 1.137 & $0.340-3.801$ \\
\hline Other & -0.363 & 0.319 & 1.293 & 1 & 0.255 & 0.696 & $0.372-1.300$ \\
\hline Condom use & 0.503 & 0.264 & 3.637 & 1 & 0.057 & 1.654 & $0.986-2.775$ \\
\hline Number of sexual partners ${ }^{\mathrm{a}}$ & 0.075 & 0.034 & 5.024 & 1 & 0.025 & 1.078 & $1.009-1.152$ \\
\hline Smoking & 0.323 & 0.345 & 0.874 & 1 & 0.350 & 1.381 & $0.702-2.715$ \\
\hline Alcohol use & -0.381 & 0.301 & 1.607 & 1 & 0.205 & 0.683 & $0.379-1.231$ \\
\hline Relationship status & 0.201 & 0.275 & 0.535 & 1 & 0.464 & 1.223 & $0.713-2.097$ \\
\hline Constant & -2.163 & 0.575 & 14.141 & 1 & 0.001 & 0.115 & \\
\hline
\end{tabular}

${ }^{a}$ During the past 12 months

Everyone in this sample was attending for an STI test so it cannot be extrapolated as to whether testers have better knowledge than non-testers, only that previous testers have better knowledge than first-time testers. It could be that the differences seen in knowledge scores among previous testers were due to them gaining knowledge at their previous test, although because the data are cross-sectional, no causation can be attributed.

In conclusion, previous STI testing was associated with STI knowledge, and a higher STI knowledge score independently predicted that an individual had previously had an STI test. 


\subsubsection{Relationship between self-assessed knowledge and measured knowledge}

The participants were asked at the start of the knowledge questions how they rated their knowledge level on a four-point scale of 'A lot', 'Some', 'A little', and 'None'. There was also an 'I don't know' option, and respondents who selected this option were removed from the following analyses. Self-assessed knowledge responses were grouped into good (A lot/Some) and bad (A little/None). Mann-Whitney U tests were run to test whether there were differences in total knowledge score between those who self-assessed their knowledge as good and those who self-assessed their knowledge as bad. Distributions of the knowledge scores for those self-assessing as good and those self-assessing as bad were not similar, as assessed by visual inspection of histograms. Knowledge scores for those that self-assessed their knowledge as good were statistically significantly higher than the scores of those who self-assessed their knowledge as bad. This relationship was found for both settings separately as well as combined (Table 6.9).

The analyses presented in this section suggest that people accurately judge their own STI knowledge level.

Table 6.9. Relationship between self-assessed knowledge and measured knowledge Student Health Services and Sexual Health Clinic Combined

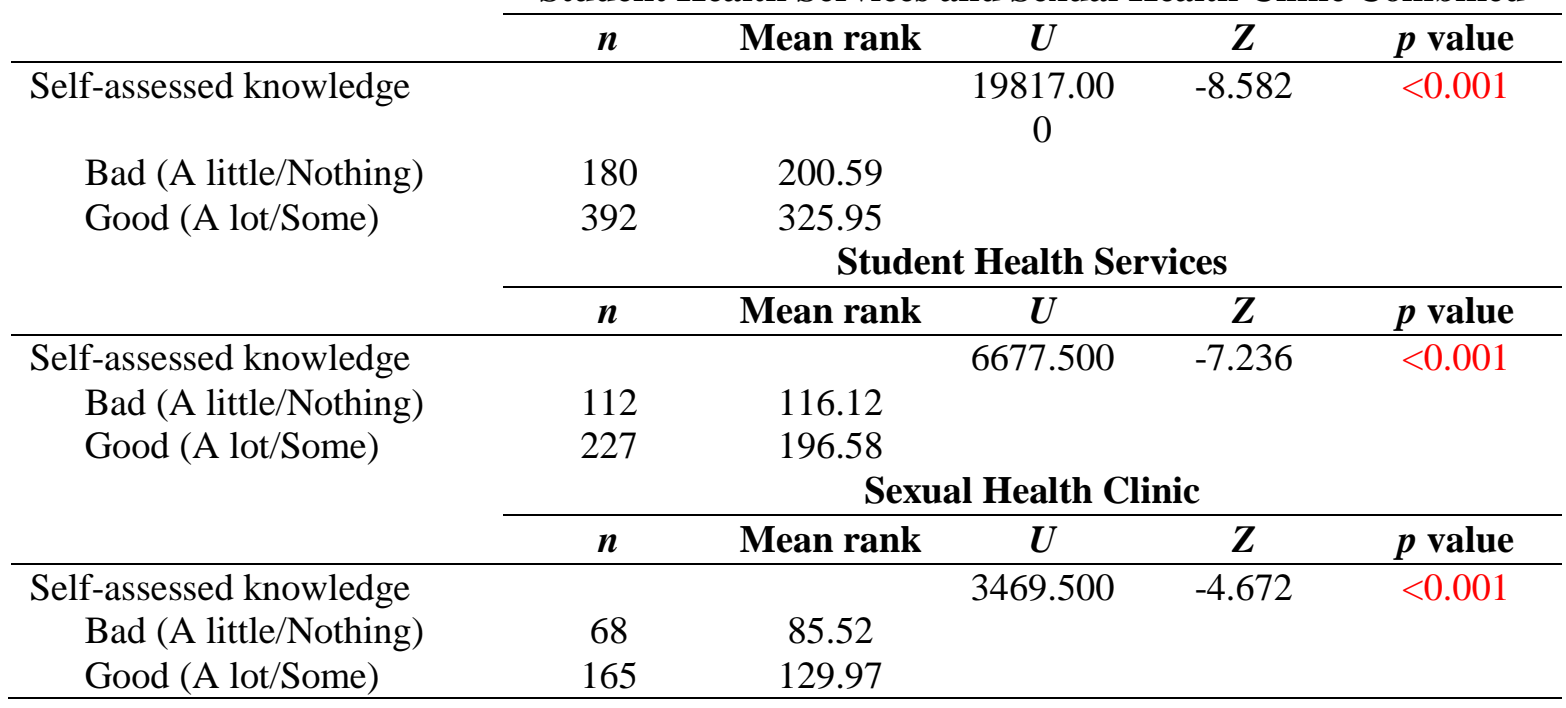




\subsubsection{Association between campaign awareness, measured knowledge and STI testing history}

To assess whether being aware of any STI campaign was associated with STI knowledge, ordinal logistic regression analyses were conducted. Individuals who stated that they were aware of an STI campaign had better STI knowledge than those who were not aware of any campaigns $(\mathrm{OR}=1.666, p=0.002,95 \% \mathrm{CI} 1.215-2.285)$. This analyses was repeated with adjustment for the demographic characteristics: gender, age, ethnicity, smoking and alcohol use, after which the relationship remained ( $\mathrm{OR}=1.830, p=0.001,95 \% \mathrm{CI} 1.301-2.575)$ (Table 6.10). However, the relationship became non-significant after further adjustment for sexual behaviour ( $\mathrm{OR}=1.576, p=0.053,95 \%$ CI $0.993-2.502)$, indicating that sexual behaviour characteristics were having an effect on the relationship between STI campaign awareness and STI knowledge. 
Table 6.10. The effect of campaign awareness on STI knowledge with adjustment for demographic characteristics $(n=528)$

\begin{tabular}{|c|c|c|c|c|c|c|c|}
\hline & $B$ & S.E & Wald & df & $p$ value & OR & $95 \% \mathrm{CI}$ \\
\hline \multicolumn{8}{|l|}{ Gender } \\
\hline Male & -0.131 & 0.1653 & 0.625 & 1 & 0.429 & 0.877 & $0.635-1.213$ \\
\hline Female & & & & & & 1 & \\
\hline \multicolumn{8}{|l|}{ Age } \\
\hline$<25$ & -0.414 & 0.2826 & 2.145 & 1 & 0.143 & 0.661 & $0.380-1.150$ \\
\hline $25-34$ & -0.109 & 0.3049 & 0.127 & 1 & 0.721 & 0.897 & $0.493-1.630$ \\
\hline $35+$ & & & & & & 1 & \\
\hline \multicolumn{8}{|l|}{ Ethnicity } \\
\hline Māori & 0.043 & 0.2863 & 0.022 & 1 & 0.881 & 1.044 & $0.595-1.829$ \\
\hline Pacific peoples & 0.069 & 0.7968 & 0.007 & 1 & 0.931 & 1.071 & $0.225-5.107$ \\
\hline Asian & -0.689 & 0.3559 & 3.753 & 1 & 0.053 & 0.502 & $0.250-1.008$ \\
\hline Other & 0.321 & 0.2052 & 2.441 & 1 & 0.118 & 1.378 & $0.922-2.060$ \\
\hline NZ European & & & & & & 1 & \\
\hline \multicolumn{8}{|l|}{ Alcohol use } \\
\hline AUDIT-C score $<5$ & -0.464 & 0.1683 & 7.610 & 1 & 0.006 & 0.629 & $0.452-0.874$ \\
\hline AUDIT-C score $\geq 5$ & & & & & & 1 & \\
\hline \multicolumn{8}{|l|}{ Smoking } \\
\hline No & -0.424 & 0.2037 & 4.336 & 1 & 0.037 & 0.654 & $0.439-0.975$ \\
\hline Yes & & & & & & 1 & \\
\hline \multicolumn{8}{|l|}{ Campaign awareness } \\
\hline Yes & 0.604 & 0.1742 & 12.037 & 1 & 0.001 & 1.830 & $1.301-2.575$ \\
\hline No & & & & & & 1 & \\
\hline
\end{tabular}

To assess whether awareness of any STI campaign had an effect on STI testing behaviour, binomial logistic regression analysis was conducted. This indicated that awareness of STI campaigns was positively associated with ever having had an STI test in the past $(\mathrm{OR}=$ $1.741,95 \%$ CI $1.033-2.933, p=0.037)$. This relationship was assessed again with adjustment for STI knowledge; determining that, after accounting for STI knowledge and other demographic variables, there was no statistically significant effect of campaign awareness on previous testing behaviour $(\mathrm{OR}=1.684,95 \% \mathrm{CI} 0.936-3.029, p=0.082)$ (Table 6.11). This suggests that the effect of campaign awareness on STI testing behaviour is mediated through STI knowledge, rather than campaign awareness directly influencing testing. However, when sexual behaviour characteristics were added into the model, the relationship became significant $(\mathrm{OR}=1.935,95 \% \mathrm{CI} 1.024-3.654, p=0.042)$. One 
explanation for this might be that sexual behaviour has a strong effect on prior testing, so without adjusting for it, there is a lot of unexplained variability which makes it difficult to detect small effects. After adjusting for sexual behaviour, the amount of unexplained variability is reduced and smaller effects (like that of campaign awareness) can be detected.

Table 6.11. The effect of campaign awareness on previous STI testing after adjustment for demographic variables and STI knowledge $(n=328)$

\begin{tabular}{|c|c|c|c|c|c|c|c|}
\hline & $\boldsymbol{B}$ & S.E. & Wald & $d f$ & $p$ value & OR & $95 \% \mathrm{CI}$ \\
\hline \multicolumn{8}{|l|}{ Gender } \\
\hline Male & & & & & & 1 & \\
\hline Female & 0.937 & 0.271 & 11.956 & 1 & 0.001 & 2.552 & $1.501-4.341$ \\
\hline \multicolumn{8}{|l|}{ Age } \\
\hline$<25$ years & & & & & & 1 & \\
\hline $25-34$ years & 0.262 & 0.313 & 0.699 & 1 & 0.403 & 1.299 & $0.704-2.398$ \\
\hline $35+$ years & 0.145 & 0.45 & 0.103 & 1 & 0.748 & 1.156 & $0.478-2.794$ \\
\hline \multicolumn{8}{|l|}{ Ethnicity } \\
\hline New Zealand European & & & & & & 1 & \\
\hline Māori & -0.161 & 0.518 & 0.096 & 1 & 0.757 & 0.852 & $0.308-2.352$ \\
\hline Pacific peoples & 0.48 & 1.295 & 0.137 & 1 & 0.711 & 1.615 & $0.128-20.445$ \\
\hline Asian & -0.018 & 0.561 & 0.001 & 1 & 0.975 & 0.983 & $0.327-2.953$ \\
\hline Other & -0.181 & 0.313 & 0.334 & 1 & 0.563 & 0.834 & $0.452-1.542$ \\
\hline \multicolumn{8}{|l|}{ Smoking } \\
\hline No & & & & & & 1 & \\
\hline Yes & 0.7 & 0.343 & 4.17 & 1 & 0.041 & 2.014 & $1.029-3.942$ \\
\hline \multicolumn{8}{|l|}{ Alcohol use } \\
\hline AUDIT-C score $<5$ & & & & & & 1 & \\
\hline AUDIT-C score $\geq 5$ & -0.19 & 0.288 & 0.434 & 1 & 0.510 & 0.827 & $0.470-1.455$ \\
\hline Total knowledge score & 0.324 & 0.08 & 16.361 & 1 & $<0.001$ & 1.383 & $1.182-1.618$ \\
\hline \multicolumn{8}{|l|}{ Campaign awareness } \\
\hline No & & & & & & 1 & \\
\hline Yes & 0.521 & 0.3 & 3.025 & 1 & 0.082 & 1.684 & $0.936-3.029$ \\
\hline Constant & -1.594 & 0.503 & 10.054 & 1 & 0.002 & 0.203 & \\
\hline
\end{tabular}

In conclusion, the analyses presented in this section suggest that awareness of STI campaigns is associated with better STI knowledge. Campaign awareness is also associated with STI testing, but STI knowledge may be mediating this relationship. 


\subsection{Discussion}

\subsubsection{Summary of main findings}

Knowledge was generally good among the participants in this study, with no significant differences in knowledge between the two study settings (SHS and SHC) even after adjustment for demographic and sexual behaviour characteristics. Questions that were answered poorly related to treatment ('gonorrhoea can usually be treated with antibiotics'), long-term health consequences ('some kinds of cancer can be caused by STIs') and the asymptomatic nature of STIs ('many STIs cause only mild or no symptoms').

Knowledge was associated with alcohol use, smoking, and ethnicity after adjustment for demographic factors, although the association between alcohol use and knowledge disappeared after adjustment for sexual behaviour variables.

Individuals who had had a previous STI test had better knowledge than those who were attending for an STI test for the first time. STI knowledge predicted ever having had a test in the past while controlling for demographic and sexual behaviour variables. As this analysis is cross-sectional, it is not possible to determine whether higher levels of knowledge encourage testing or whether previous testing leads to higher levels of knowledge.

Being aware of any STI campaign predicted having had an STI test in the past. This association appeared to be somewhat mediated through STI knowledge.

\subsubsection{Comparison to other literature}

\subsubsection{STI knowledge level}

Questions that were answered poorly related to treatment, long-term health consequences, and the asymptomatic nature of STIs. Similar trends have also been found in other studies (Devonshire et al., 1999, Mason, 2005, Greaves et al., 2009, Lorimer and Hart, 2010).

Direct comparison of the STI knowledge of participants in the current study to participants in the Second Australian Study of Health and Relationships (ASHR2) is possible for a small number of questions which were identical in both questionnaires (Grulich et al., 2014). The two samples had very similar knowledge about genital herpes, with the correct answer to the question 'once a person has caught genital herpes, they will always have the virus' being 
given by $73 \%$ of the participants in the current study and $72 \%$ of participants in the ASHR 2 . However, the participants in the current study had slightly better knowledge of STI sequelae as measured by the question 'chlamydia can lead to infertility in women' ( $75 \%$ correct vs $70 \%$ correct) and were also more likely to answer the question 'chlamydia affects only women' correctly ( $85 \%$ vs $57 \%$ ). It is likely that these differences in knowledge scores are due to differences between the two populations. The ASHR2 is a population survey of adults in Australia, and is likely to be more representative of a general population. Additionally, the current study excluded people who had never been sexually active whereas the ASHR2 sample will have included these individuals, and analysis of data from the current study and others (Kang et al., 2014) demonstrates that people who have not been sexually active tend to have lower STI knowledge.

\subsubsection{Sociodemographic predictors of STI knowledge}

Knowledge was associated with alcohol use, smoking, and ethnicity. Previous studies have reported that women tend to have better STI knowledge than men and some have hypothesised that this may be due to women more often attending healthcare for sexual health related matters such as contraception and cervical screening where they would be likely to receive accurate STI information (Kellock et al., 1999, Greaves et al., 2009, Langille et al., 2009, Agius et al., 2010, Lorimer and Hart, 2010, Malacova et al., 2011, SamkangeZeeb et al., 2011, Simpson et al., 2015). However, no statistically significant association between gender and STI knowledge was found in this study.

There was no difference in STI knowledge by age group in the current study. Previous studies have reported mixed results with regards to the effect of age on STI knowledge. Grulich et al. (2014) found that those who were younger had better STI knowledge, whereas Malcova et al. (2011) reported that older respondents tended to have better knowledge. To add confusion, Mason (2005) found that men in the middle age-bands (from 20-24 to 45-54 age groups) had a greater awareness of all the STIs than those in the youngest (16-19) and eldest groups (55+). Lastly, similar to this study, others found no association between knowledge and age (Devonshire et al., 1999).

Level of education was not associated with STI knowledge in the current study. Although this may be because the sample was generally a highly educated group, with many people 
either currently studying for or having already gained a tertiary qualification. One measure which was not made in the current study was whether participants had ever received any school-based sex education. Previous studies have suggested that sex education at school is associated with higher levels of knowledge (Grulich et al., 2014, Simpson et al., 2015).

Ethnicity was related to STI knowledge in the current study; those who identified as Asian had poorer knowledge than those who identified as New Zealand European. The same pattern has been found in Australian studies, where Asian-born university students have consistently poorer HIV/STI knowledge scores than Australian-born students (Song et al., 2005, Simpson et al., 2015). These data suggest that educational campaigns and materials need to be made available which are culturally relevant for people of Asian ethnicity.

Alcohol use and smoking were both related to sexual health knowledge, as has been reported in previous research (Andersson-Ellstrom and Milsom, 2002), but this relationship disappeared after adjustment for sexual behaviour variables. This suggests that the effect of alcohol and smoking on knowledge are mediated through risky sexual behaviour, indicating a general high-risk behaviour influence. Drinking alcohol has been shown to be involved in

making risky sexual behaviour choices (Aicken et al., 2011b, Rehm et al., 2012, Connor et al., 2015), although the relationship between these behaviours is complex (Connor et al., 2015).

\subsubsection{STI knowledge and risky sexual behaviour}

It could be hypothesised that greater knowledge about STIs would be associated with less high-risk sexual behaviour, but no association was observed in the current study after adjustment for covariates. A similar finding has also been reported in other studies, including that of over 700 young people attending sporting clubs in Victoria, Australia (Kong et al., 2010), and another of adults in Bhutan (Norbu et al., 2013). It appears that knowledge of risks or methods of prevention don't actually translate into behaviour change in the case of risky sexual behaviour (Crosby et al., 2000, Sekirime et al., 2001, Lazarus et al., 2010). In fact, some studies have reported that the association of STI knowledge with risk behaviour is in the opposite direction, where a higher level of knowledge is associated with greater risky behaviour (Andersson-Ellstrom and Milsom, 2002, Simpson et al., 2015). This suggests that the most at-risk are the most informed of the dangers, but do not alter their sexual behaviour. 
The results of the current study showed that the number of sexual partners in the previous year weakly correlated with knowledge score, where those with a higher number of partners were more likely to have a higher knowledge score. However, there was no statistically significant association observed between these two variables in the adjusted regression analyses.

\subsubsection{STI knowledge and STI testing history}

These results reflect those from a previous study at a university in England where students who had ever been screened for chlamydia had a higher mean knowledge score than those who had not been screened (Greaves et al., 2009). Similarly, in a study of MSM in Australia, Pedrana et al. (2012) found that STI knowledge was independently associated with having had any STI test within the past six months (Prevalence Risk $=1.2 ; 95 \% \mathrm{CI}=1.0-1.4$ ). A Canadian study of students at eight universities reported a similar relationship, where those with a higher level of sexual health knowledge were more likely to have ever had an STI test (Cragg et al., 2016). However, in another Canadian study, this time among high-school students, there was no association between knowledge score and having had an STI test, although test history was restricted to the previous year (Langille et al., 2009). The knowledge questionnaire used in the Canadian high-school study was focussed on chlamydia and only two questions corresponded to questions used in the current study, meaning the questionnaires were likely measuring different constructs. This may explain why the Canadian high-school study did not find an association while the current study did. The knowledge questionnaire used in the English study by Greaves et al. (2009) was also focussed solely on chlamydia, but there was slightly more overlap in questions to those asked in the current study which may be why the association between knowledge and testing history was similar. Questionnaires were not presented in the papers by Pedrana et al. (2012) or Cragg et al. (2016) so comparison of the STI knowledge measures with the knowledge measure in the current study was not possible.

In addition to knowledge, female gender and having a higher number of sexual partners also independently predicted previous STI testing. These results agree with what has been found in previous studies, both internationally, and in New Zealand (Morgan and Bell, 2009, Morgan and Haar, 2009, Dickson et al., 2015, Kerry et al., 2015, Cuffe et al., 2016). 


\subsubsection{Self-assessed knowledge and measured knowledge}

Individuals in the current study appeared to self-assess their own knowledge level well. This finding agrees with previous research in New Zealand; a study of HIV/AIDS knowledge conducted in 1994 among school-aged students in Auckland found that self-estimation of knowledge was comparable to actual knowledge level (Duncan and Bergen, 1997).

\subsubsection{Campaign awareness, STI knowledge and STI testing history}

This study demonstrated that individuals who were aware of any STI campaign, either now or in the past, had better STI knowledge. As better STI knowledge was also associated with previous STI testing, it is possible that campaigns may encourage testing. However, the cross-sectional design of this study prevents the testing of this hypothesis. A few studies have attempted this (Tyden et al., 1994, Tyden et al., 1998, McClean et al., 2000, Lim et al., 2012). For example, Lim et al. (2012) recruited young people at a music festival to receive SMS and email messages about STIs, and reported increased STI knowledge in males and females and increased self-reported STI testing in females in the intervention group. However other studies have not shown such promising results; educational campaigns targeted at university students in Sweden were successful at improving knowledge about STIs, but did not increase the number of people attending for an STI test (Tyden et al., 1994, Tyden et al., 1998).

\subsubsection{Strengths and limitations}

Many studies have employed a short set of questions to measure STI knowledge, however, few have made any assessment of validity. The set of seven STI knowledge questionnaires used in the current study were compared to a gold-standard comprehensive STI knowledge tool in order to measure criterion validity. The two were shown to be strongly correlated, providing confidence that the seven-item knowledge questionnaire is measuring a similar construct to the comprehensive measure. However, further assessments of the validity of the tool were not carried out, such as test-retest reliability assessment. This could be the focus of future work.

The response rate among the SHC sample was high (73.4\%) but unfortunately it was not possible to calculate a response rate for the SHS sample as the number of questionnaires offered was not recorded. Participants were generally comparable to the wider populations 
for both samples, providing confidence that the research may be considered representative of these clinical populations. These populations were chosen as they are at high-risk of STIs and so are the target group for STI testing. Although a strength of this research is that samples from two populations have been compared and contrasted, generalisability of these results should be made with caution, as university students and attendees at SHCs are not representative of the general population (Catchpole et al., 1997).

Questionnaires are vulnerable to false answers being given by participants. To help reduce social desirability bias, the questionnaire was designed to be self-completed and anonymous (unless participant chose to provide identifying information).

Another limitation of this study is that it is possible that participants could have guessed the answers to the knowledge questions. To avoid this from happening, an 'I don't know' option was included and individuals were encouraged to select this if they did not know the answer, rather than to guess.

Lastly, the cross-sectional study design limits understanding of the direction of causal effect. Therefore, it cannot be concluded from this data whether the association between STI knowledge and testing history is due to STI knowledge influencing people to find out their STI status, or if having an STI test leads to better knowledge through, perhaps, educational discussions with the health professional. More prospective studies of STI knowledge and testing behaviour are required in order to understand this association in more detail.

\subsubsection{Implications and importance}

These data add to the evidence-base that better STI knowledge is not associated with less risky sexual behaviour. Although this is discouraging, the findings also suggest that better knowledge is associated with previous STI testing. More research into this is required but the mechanism is plausible. Applying rationale (knowledge) to an emotionally-charged momentary situation, for example when about to have sex, is much more difficult than rational thinking over a longer period of time, such as after the event when considering whether to have an STI test. Therefore, it may be that improving STI knowledge is more useful for encouraging secondary prevention than for increasing primary prevention. As STI 
knowledge is modifiable through educational interventions (Lim et al., 2012), there is potential for improving healthcare-seeking behaviour via this means.

The participants in this study self-rated their STI knowledge accurately, indicating that people have a reasonable appreciation of their individual knowledge level. This suggests that we should be concerned when young people declare that they have less than adequate knowledge about STIs, as was seen in the qualitative study (Chapter 5), and provide them with a better education and understanding.

\subsection{Chapter summary and next steps}

This chapter has supported the finding from Chapter 5 that STI knowledge is associated with STI testing behaviour. The combination of qualitative and quantitative evidence strengthens the position that STI knowledge positively influences STI testing behaviour.

From this chapter, it is clear that participants had variable knowledge about STI sequelae. STI sequelae have not been explored thus far in this thesis. The following chapter describes a systematic review assessing the incidence of ReA after STI. ReA is an important health outcome of STI because it can occur just a few weeks after infection, which is much sooner than the majority of STI sequelae which tend to be viewed as long-term health outcomes. As such, ReA presentation is potentially another opportunity in which to diagnose and subsequently treat an STI early in infection progression, thus minimising onwards transmission. However, it is not currently known how often this complication occurs. The following chapter aims to provide this information. 


\section{Chapter 7 The incidence of sexually acquired reactive arthritis: a systematic literature review}

\subsection{Chapter Overview}

Previous chapters have focussed on healthcare-seeking behaviour of individuals related specifically to going for an STI test. Another pathway to care for STIs is when health issues which are sequelae of STIs become apparent to an individual, such as chronic pelvic pain or fertility issues, and they seek healthcare for these instead. A lesser known sequela is reactive arthritis (ReA), which can occur at any time after an individual has contracted an STI. It is yet unknown how common ReA after STI is, so it is not clear if it has the potential to be a significant pathway to identifying previously undiagnosed STIs. The work that follows was published in Clinical Rheumatology, 2016; 35(11): 2639-2648. Initials used in this chapter: $\mathrm{HD}=$ Hayley Denison, $\mathrm{BC}=$ Beth Curtis, $\mathrm{RG}=$ Rebecca Grainger .

\subsection{Abstract}

Objective: Reactive arthritis ( $\operatorname{ReA})$ is an inflammatory spondyloarthritis occurring after infection at a distant site. Chlamydia trachomatis is proposed to be the most common cause of ReA, yet the incidence of sexually acquired ReA (SARA) has not been well established. We therefore carried out a systematic literature review to collate and critically evaluate the published evidence regarding the incidence of SARA.

Methods: MEDLINE and EMBASE databases were searched using free-text and Medical Subject Headings (MeSH) relating to infection and ReA. The title and abstract of articles returned were screened independently by two reviewers and potentially relevant articles assessed in full. Data was extracted from relevant articles and a risk of bias assessment carried out using a validated tool. Heterogeneity of study methodology and results precluded meta-analysis.

Results: The search yielded a total of 11680 articles, and a further 17 were identified from review articles. After screening, 55 papers were assessed in full, from which 3 met the relevant inclusion criteria for the review. The studies reported an incidence of SARA of $3.0 \%-8.1 \%$ and were found to be of low to moderate quality. 
Conclusions: More studies are required to address the lack of data regarding the incidence of SARA. Specific and sensitive classification criteria must be developed in order for consistent classification and valid conclusions to be drawn. In clinical practice, it is recommended clinicians discuss the possibility of ReA developing at the time of STI diagnosis, and to encourage patients to return if they experience any relevant symptoms.

\subsection{Introduction}

Reactive Arthritis ( $\operatorname{ReA})$ is an inflammatory spondyloarthritis occurring after infection at a distant site. It typically occurs in young adults, one to four weeks after infection and can affect axial or peripheral joints and periarticular tissues (Hamdulay et al., 2006). While ReA is usually mild and self-limiting, up to $18 \%$ of those affected develop a chronic arthritis (Hannu et al., 2006). In some, but not all studies, the HLA-B27 gene is associated with increased severity and chronicity of ReA (Pope et al., 2007).

It is reported that ReA can be triggered by infection with bacteria, viruses and parasites (Ozgul et al., 2006, Espinoza and Garcia-Valladares, 2013), with the most frequent microbial triggers infecting the gastrointestinal or genitourinary tracts (Hamdulay et al., 2006).

Chlamydia trachomatis is proposed to be the most common cause of $\operatorname{ReA}$ (Keat et al., 1987,

Kvien et al., 1994, Ozgul et al., 2006, Morris and Inman, 2012, Singh and Karrar, 2014), with other sexually transmitted infections (STIs) including Neisseria gonorrhoeae (distinct from its role in septic gonococcal arthritis), and Ureaplasma urealyticum also implicated (Carlin et al., 2014). ReA triggered by an STI is referred to as sexually acquired reactive arthritis (SARA) (Carlin et al., 2014).

The association of arthritis with STIs has been long recognised. Hippocrates was probably the first to link the presence of arthritis and infection in the genitourinary tract when he observed, at a time when the term "gout" was used to refer to acute arthritis, that "A youth does not suffer from gout until after sexual intercourse" (Lloyd, 1978, Iglesias-Gammara et al., 2005). The first report of joint involvement after venereal disease was in 1715 by Musgrave, and the first clear description given by Swediaur in 1798 and 1809 (Storey, 1998), 
though the term "reactive arthritis", referring to nonpurulent arthritis associated with infection, wasn't introduced until 1969 (Ahvonen et al., 1969, Kuipers et al., 1999).

Despite this long history and extensive literature, the incidence rate of ReA after STI is not well established. A large proportion of ReA cases are probably unrecognised due to mild symptoms and resolution without treatment (Hamdulay et al., 2006). It is also unclear how long after infection ReA can occur, and which infections can cause ReA (Townes, 2010). This is complicated by conflicting data regarding pathophysiology, where the inciting events could include viable bacteria in the synovium or an immune-mediated mechanism in response to chlamydial antigenic debris at the synovial site (Inman et al., 2000, Owlia and Eley, 2010, Morris and Inman, 2012, Selmi and Gershwin, 2014). As such, there are no validated diagnostic criteria for ReA for clinical use (Hannu et al., 2006) and no universally accepted classification criteria for clinical research which leads to inconsistency in reported incidence rates in the literature (Pacheco-Tena et al., 1999, Singh and Karrar, 2014).

Despite this, many studies have attempted to determine an incidence rate for ReA. The systematic literature reviews to date have focussed on ReA triggered by enteric infection with ReA rates reported as 2.86\% - 5.8\% (Ajene et al., 2013, Keithlin et al., 2014, Keithlin et al., 2015). Despite chlamydia being widely stated as the most common infection causing ReA, there has not been a systematic review assessing the incidence of SARA. We therefore carried out a systematic literature review to collate and critically evaluate the published evidence regarding the incidence of SARA.

\subsection{Methods}

The methods recommended by the Centre for Reviews and Dissemination (CRD), University of York (Centre for Reviews and Dissemination, 2009) were used and the data reported following guidelines set out in the Preferred Reporting Items for Systematic reviews and Meta-Analysis (PRISMA) statement (Moher et al., 2009). The protocol for this study is available on request from the authors. 


\subsubsection{Eligibility criteria}

Studies were included that reported an incidence or proportion of ReA cases after any sexually-associated infection, with the exception of human immunodeficiency virus (HIV). HIV was excluded as a trigger for SARA as although ReA is reported in people with HIV infection, it is not clear whether this is due to HIV per se, or as immunocompromised status results in opportunistic infections (such as other STIs) which trigger ReA (Lawson and Walker-Bone, 2012). The current epidemiological data regarding HIV and ReA are too complex and variable to draw conclusions about the relationship between HIV infection and ReA (Lawson and Walker-Bone, 2012). As this literature has already been comprehensively reviewed elsewhere, we decided to exclude studies of HIV and ReA from this review.

All prospective studies assessing for ReA after infection, where ReA could be assessed at the time of diagnosis of infection or at follow-up were included. All time periods and populations were included, including any age-group, gender, or country. Only studies with laboratory confirmation of the infection were included to avoid biasing rates due to including participants with infection based on self-report. Studies that identified ReA and retrospectively tested for STIs were excluded, as were articles not written in English language, as translation facilities were not available.

\subsubsection{Information sources}

MEDLINE and EMBASE databases (1946-present) were searched to produce a database of abstracts, eliminating any duplicate articles.

\subsubsection{Search strategy}

Search terms were generated on the basis of the research objective and included infection terms and ReA terms. Both Medical Subject Headings (MeSH) terms and free-text terms were used. Search terms including enteropathogenic causes of ReA were included to identify papers which may have included STI organisms but not included the title or abstract. Reiter's syndrome terms were included, as this has been used synonymously with ReA in the past. We sought the input of an information specialist to assist in developing the search strategy to maximise its efficiency at returning relevant articles. The full electronic search strategy is presented in Figure 7.1. This search was conducted in June 2014 and saved in the Ovid 
system for autoalerts to be emailed to the reviewers monthly. These were then screened for additional articles until the end of December 2015. The references of relevant review articles were also checked for studies that may be applicable to the present review.

(((exp arthritis, reactive/) OR ((reactive arthritis or reiter* syndrome or reiter* disease)ti,ab.)) OR
(((exp arthritis, infectious/) OR ((reactive arthritis or reiter* syndrome or reiter* disease* or OR
"infectious arthritis")ti,ab.)) AND ((exp sexually transmitted diseases/ OR exp chlamydia
infections/ OR exp chlamydia trachomatis/ OR exp campylobacter/ OR exp campylobacter
infections/ OR exp salmonella/ OR exp salmonella infections/ OR exp shigella/ OR exp
gastroenteritis OR exp Yersinia Infections/ OR exp Yersinia/) OR ((sexually transmitted infection*
or sexually transmitted disease* or STI or STD or chlamydia or campylobacter or salmonella or
shigella or gastroenteritis or yersinia or yersina)ti,ab.))) and humans/) or (((exp arthritis, reactive/)
OR ((reactive arthritis or reiter* syndrome or reiter* disease)ti,ab.)) OR (((exp arthritis,
infectious/) OR ((reactive arthritis or reiter* syndrome or reiter* disease* or OR "infectious
arthritis")ti,ab.)) AND ((exp sexually transmitted diseases/ OR exp chlamydia infections/ OR exp
chlamydia trachomatis/ OR exp campylobacter/ OR exp campylobacter infections/ OR exp
salmonella/ OR exp salmonella infections/ OR exp shigella/ OR exp gastroenteritis OR exp Yersinia
Infections/ OR exp Yersinia/) OR ((sexually transmitted infection* or sexually transmitted disease*
or STI or STD or chlamydia or campylobacter or salmonella or shigella or gastroenteritis or yersinia
or yersina)ti,ab.))) not (humans/ or animals/))

Figure 7.1. Full electronic search strategy

\subsubsection{Study selection}

Two reviewers (HD and BC) independently screened the title and abstract of articles returned by the search with a low threshold for inclusion. The final inclusion lists of the two reviewers were then compared and full text of all relevant articles were obtained. The articles were then assessed by one reviewer (HD) to determine if criteria for inclusion in the review were met, and this list was checked by the second reviewer (BC). In case of disagreement, a third reviewer (RG) gave a deciding opinion.

\subsubsection{Data collection}

Data was extracted from each article by one reviewer (HD) into tables, which were then checked for accuracy by a second reviewer (RG). Information extracted included the following: general details (the reviewer and date); study details (type of study, inclusion/exclusion criteria); study population description; methodology of infection 
diagnosis; methodology of ReA diagnosis; analysis (statistical techniques used, losses to follow-up); and research results. The principal summary measure recorded to use in this review was incidence rate of $\mathrm{ReA}$, or if no incidence rate was calculated, proportion of infected cases developing ReA.

\subsubsection{Risk of bias in individual studies}

To assess the methodological quality of the primary research, a risk of bias assessment was conducted independently by two reviewers (HD and BC) using the RTI (Research Triangle Institute) item bank (Viswanathan and Berkman, 2012). From the 29 items in the RTI item bank the 14 relevant items were selected, according to the authors' instructions (Table 7.3). For each item, criteria relevant to determining the risk of bias were specified to aid the reviewers. An overall judgement of the risk of bias for each study was made based on whether the biases assessed were likely to seriously alter the results. Disagreements were resolved by discussion to reach consensus. The assessment information was used in data synthesis to interpret study results on a background of methodological quality.

\subsubsection{Synthesis of results}

Heterogeneity of study methodology and results precluded meta-analysis so data were summarised in tabular and narrative form.

\subsection{Results}

\subsubsection{Study selection}

No systematic review addressing the research objective of the present paper was discovered. The database searches yielded a total of 11,680 articles, and a further 17 were identified through screening the references of relevant review articles. After title and abstract screening, 11,642 articles were excluded as they were not relevant. Fifty-five papers were sought in full for further assessment. Three papers were only published as conference abstracts. Forty-nine papers were excluded after assessment of the full text because of the following: they were review papers and did not contain original research $(n=2)$; they were not available in English language $(n=9)$; exposure infection was not sexually acquired $(n=8)$; they had a 
retrospective design $(n=7)$; or they did not report $\operatorname{ReA}$ incidence $(n=13)$. Studies where the infection was not laboratory confirmed were also excluded $(n=9)$. An additional paper (Catterall, 1958) was excluded because it reported the number of patients with Reiter's disease and the number of patients with non-specific genital infection in a group of patients with uveitis, but did not report the relationship of interest (number of patients with genital infection and ReA from the total cohort). Thus, three articles (Keat et al., 1978, Rich et al., 1996, Carter et al., 2013) were retained for the systematic review (Figure 7.2). 


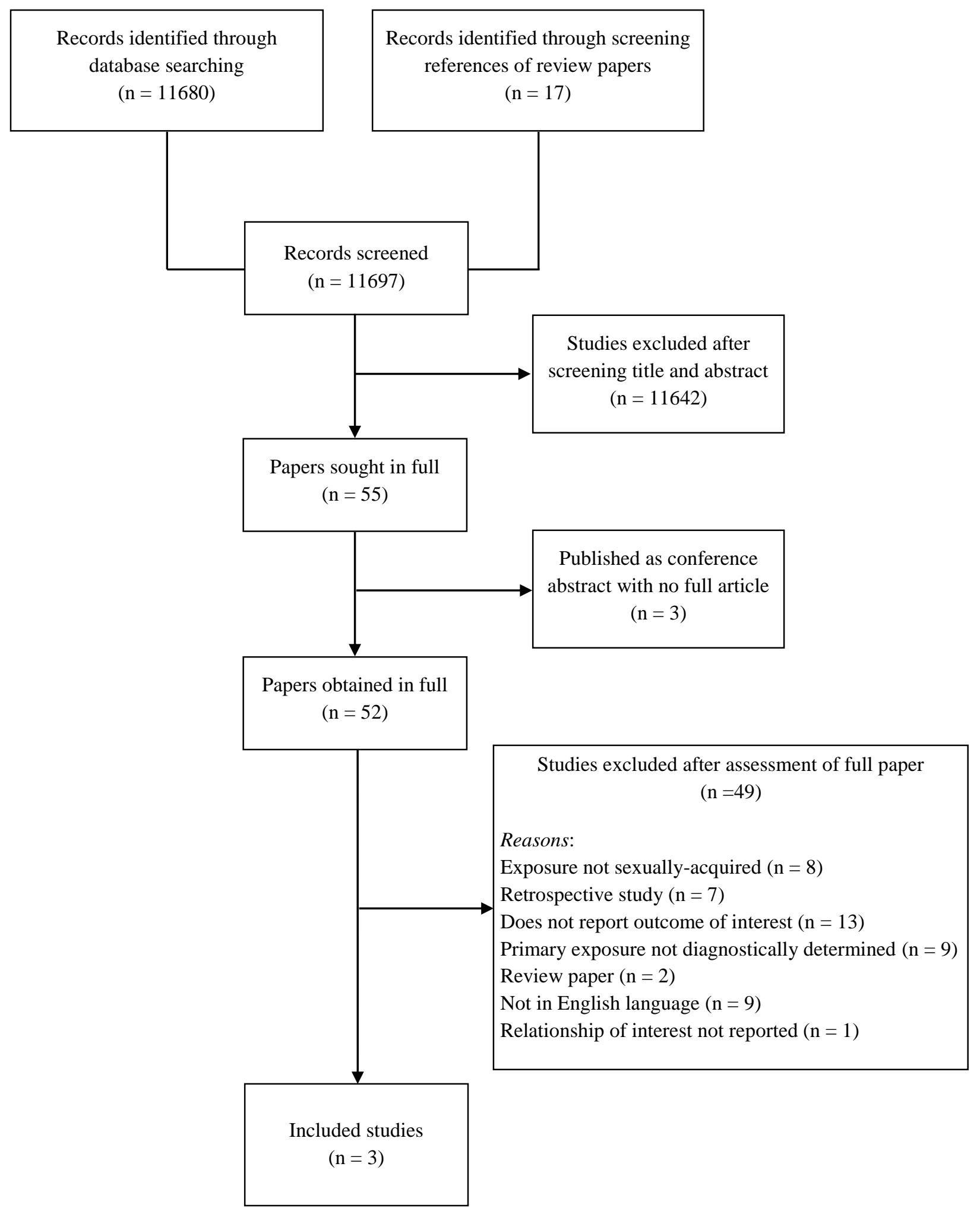

Figure 7.2. Flow diagram depicting the identification and selection of relevant papers 


\subsubsection{Study characteristics (Table 7.1)}

The three papers were published over a 35 year period $(1978,1996,2013)$, two in the USA (Rich et al., 1996, Carter et al., 2013) and one in the UK (Keat et al., 1978). The studies were situated in STI (Keat et al., 1978, Rich et al., 1996) or communicable disease clinics (Carter et al., 2013). The mean age of the men and women in the two American studies was 24.4 years (Carter et al., 2013) and 26 years (Rich et al., 1996). Ethnicities varied depending on clinic clientele; participants in the Alabama study were predominantly African American (98.5\%) (Rich et al., 1996) and in the Florida study African American (63\%), Hispanic (22\%), white (13\%) and other (1.3\%) (Carter et al., 2013). The UK study recruited heterosexual men only and provided no data on age or ethnicity (Keat et al., 1978).

The primary exposure in the studies varied. The oldest paper, Keat et al. (1978) included 531 men with non-specific urethritis while cultures were taken to confirm chlamydia in some participants $(n=384)$. Rich et al. (1996) recruited patients who were being treated with doxycycline for possible or proven chlamydia infection $(n=271)$ and included patients with gram stain or cell culture diagnosis confirmation of genital infection/inflammation $(n=217)$ as the denominator for the ReA incidence analysis. This group included men diagnosed with gonococcal urethritis and nongonococcal urethritis, and women diagnosed with $N$. gonorrhoeae and C. trachomatis. Carter et al. (2013) only recruited patients who tested positive for $C$. trachomatis $(\mathrm{n}=365) ; 149$ were followed up for assessment of $\operatorname{ReA}$ symptoms.

Two papers identified ReA cases using a screening questionnaire prompting further evaluation (Rich et al., 1996, Carter et al., 2013) (Table 7.2). One of these used a questionnaire derived from a validated questionnaire (Thomson et al., 1993) and was administered by rheumatology subspecialty residents by phone at six week follow-up only (Carter et al., 2013). Rich et al. (1996) used a paper questionnaire completed by participants at initial clinic visit and via mail at six weeks. In both studies a positive answer to screening questions prompted invitation to evaluation in person by a rheumatologist. In the study by Carter et al., participants declined to attend a rheumatology evaluation, so the protocol was amended to telephone evaluation only, with all participants with a positive six-week 
telephone questionnaire defined as 'possible/probable ReA' (Carter et al., 2013). The third paper had no detailed data about the process of evaluation (Keat et al., 1978).

\subsubsection{Risk of bias assessment}

The risk of bias of the articles included in this review was considered medium to high; therefore, the interpretation of the results of these articles is limited (Table 7.3). Key biases included attrition bias, selection bias, information bias, performance bias and reporting bias. The articles failed to report how many participants were approached to participate in the studies, and how they were selected. Details about the timing of recruitment and assessment were also lacking. Details about participant selection were too vague to interpret to what extent their selection protocol may have affected results.

Infection diagnosis was made using objective and valid diagnostic tools in all studies, either by culture, gram stain or Nucleic Acid Amplification Test (NAAT). However, as the studies were published from 1978 to 2013 the diagnostic techniques vary widely in method and sensitivity, preventing direct comparison.

Only one study completed assessment of ReA by a rheumatologist with physical evaluation (Rich et al., 1996). Telephone assessment to define ReA, used in the study by Carter et al. (2013), could have introduced bias and limits comparability between the two studies. The final paper did not discuss how ReA was assessed (Keat et al., 1978).

In the two studies that reported the number of participants followed-up, there was a high attrition rate which it is likely to have introduced bias (Rich et al., 1996, Carter et al., 2013). Reliance on return of questionnaires by mail may have resulted in over-reporting of incidence. The article by Keat et al. (1978) especially lacked sufficient information about the methods, making it difficult to accurately assess many items on the risk of bias tool.

\subsubsection{Outcome measure definition}

One study used an established criteria for ReA (Carter et al., 2013), the European Spondyloarthropathy Study Group (ESSG) criteria (Dougados et al., 1991). Plain radiographs of the sacroiliac joints (one ESSG criterion) was omitted as the evaluation was by telephone. Two patients were classified as ReA cases despite not fully meeting the classification criteria. 
The other two studies did not use any classification criteria, Rich et al. (1996) listed ReA features assessed by rheumatologists, and Keat et al. (1978) gave a brief definition of SARA in the introduction.

\subsubsection{ReA incidence rate}

A meta-analysis was not possible due to the small number of relevant studies, heterogeneity of methodology between studies, medium to high risk of bias, and the differing ReA diagnostic criteria used. A narrative summary of results is presented.

Rich et al. (1996) reported that nine of 217 patients (4.1\%) had objective ReA features as assessed by a rheumatologist. Carter et al. (2013) reported an incidence rate almost double that of Rich et al. (1996), with 12 out of 149 (8.1\%) participants having symptoms consistent with ReA. Ten of these 12 participants met the ESSG diagnostic criteria for spondyloarthritis; the 2 participants that did not fulfil these criteria were judged to have ReA based on expert opinion. The ratio of female-to-male cases was about 1:1 in both of these mixed sex studies. Keat et al. (1978) reported that 16 patients developed ReA from the 531 studied, giving an incidence of $3 \%$. 
Table 7.1. Characteristics of included studies

\begin{tabular}{|c|c|c|c|c|c|c|c|c|c|}
\hline First author & $\begin{array}{l}\text { Year of } \\
\text { publication }\end{array}$ & $\begin{array}{l}\text { Study } \\
\text { location }\end{array}$ & $\begin{array}{l}\text { Type of } \\
\text { study }\end{array}$ & Study size & Study population & $\begin{array}{l}\text { Primary } \\
\text { exposure(s) } \\
\text { (Infection) }\end{array}$ & Infection diagnosis & $\begin{array}{l}\text { Incidence of } \\
\operatorname{Re} A\end{array}$ & $\begin{array}{l}\text { Proportion of } \\
\text { ReA cases with } \\
\text { asymptomatic } \\
\text { infection }\end{array}$ \\
\hline Carter & 2013 & $\begin{array}{l}\text { Florida, } \\
\text { USA }\end{array}$ & Prospective & $\begin{array}{l}149 \text { (from } 365 \\
\text { enrolled) }\end{array}$ & $\begin{array}{l}\text { Adults attending } \\
\text { communicable disease } \\
\text { clinic testing positive } \\
\text { for Chlamydia } \\
\text { trachomatis }\end{array}$ & $\begin{array}{l}\text { Chlamydia } \\
\text { trachomatis }\end{array}$ & $\begin{array}{l}\text { Laboratory diagnosis by } \\
\text { gram stain, cell culture or } \\
\text { NAAT }\end{array}$ & $\begin{array}{l}12 / 149 \\
(8.1 \%)\end{array}$ & $8 / 12(66.7 \%)$ \\
\hline Rich & 1996 & $\begin{array}{l}\text { Alabama, } \\
\text { USA }\end{array}$ & Prospective & 217 & $\begin{array}{l}\text { Adults attending a } \\
\text { sexually transmitted } \\
\text { diseases clinic being } \\
\text { treated with } \\
\text { doxycycline for a } \\
\text { possible or proven } \\
\text { Chlamydia } \\
\text { trachomatis infection }\end{array}$ & $\begin{array}{l}\text { Genital } \\
\text { infection/ } \\
\text { inflammation }\end{array}$ & $\begin{array}{l}\text { Laboratory diagnosis by } \\
\text { cervical cell culture for } \\
\text { Neisseria gonorrhoeae and } \\
\text { Chlamydia trachomatis. In } \\
\text { men, a gram-stained } \\
\text { urethral smear and a } \\
\text { urethral Neisseria } \\
\text { gonorrhoeae culture were } \\
\text { obtained. Chlamydia } \\
\text { trachomatis genital cultures } \\
\text { were obtained for every } \\
\text { patient who had objective } \\
\text { ReA features }\end{array}$ & $\begin{array}{l}9 / 217 \\
(4.1 \%)\end{array}$ & $7 / 9(77.8 \%)$ \\
\hline Keat & 1978 & UK & Prospective & 531 & $\begin{array}{l}\text { Heterosexual men } \\
\text { attending sexually } \\
\text { transmitted diseases } \\
\text { clinic with new } \\
\text { episodes of urethritis }\end{array}$ & $\begin{array}{l}\text { Non-specific } \\
\text { urethritis. } \\
\text { Cultures for } \\
\text { Chlamydia } \\
\text { trachomatis } \\
\text { taken to } \\
\text { investigate } \\
\text { association } \\
\text { with ReA }\end{array}$ & $\begin{array}{l}\text { Urethral smear. Non- } \\
\text { specific urethritis if over } 10 \\
\text { polymorphs found in } \geq 3 \\
\text { consecutive high-power } \\
\text { fields (magnification x 600), } \\
\text { if microscopy, culture, and } \\
\text { serology excluded } \\
\text { gonorrhoea and syphilis }\end{array}$ & $\begin{array}{l}16 / 531 \\
(3.0 \%)\end{array}$ & No details \\
\hline
\end{tabular}


Table 7.2. ReA diagnosis methodology of included studies

\begin{tabular}{|c|c|c|c|}
\hline Study & Classification criteria used & Screening tool used & Full evaluation \\
\hline Carter et al. 2013 & $\begin{array}{l}\text { The European Spondylarthropathy Study } \\
\text { Group (ESSG) criteria (excluding plain } \\
\text { radiographs of the sacroiliac joints) } \\
\text { (Dougados et al., 1991). }\end{array}$ & $\begin{array}{l}\text { Standardised questionnaire of } 13 \text { questions } \\
\text { by telephone by rheumatology sub-speciality } \\
\text { residents, including new arthritis symptoms } \\
\text { or other symptoms of ReA such as } \\
\text { conjunctivitis, uveitis and enthesitis. }\end{array}$ & $\begin{array}{l}\text { Details obtained for any positive responses } \\
\text { to the questionnaire during the telephone } \\
\text { interview. In-person review declined by } \\
\text { participants. }\end{array}$ \\
\hline Rich et al. 1996 & $\begin{array}{l}\text { Features of ReA considered: oligoarthritis, } \\
\text { enthesitis in any location, inflammatory axial } \\
\text { pain or mucocutaneous inflammation } \\
\text { (conjunctivitis, oral ulcers, circinate balanitis, } \\
\text { or keratoderma blennorrhagica). }\end{array}$ & $\begin{array}{l}\text { A standardised questionnaire of } 8 \text { questions } \\
\text { derived from QUEST } 2 \text { (Questionnaire } \\
\text { Utilizing Epidemic Spondyloarthropathy } \\
\text { Traits) (Thomson et al., 1993). In the last } 60 \\
\text { days: joint pain, joint swelling, morning } \\
\text { stiffness, heel pain, inflammation of the } \\
\text { eyes, oral mucosal lesions, or skin rashes } \\
\text { involving the palms or soles. }\end{array}$ & $\begin{array}{l}\text { Patients with positive answers to any } \\
\text { question evaluated by a rheumatologist by } \\
\text { history and physical examination with } \\
\text { particular attention to features of ReA. }\end{array}$ \\
\hline Keat et al. 1978 & $\begin{array}{l}\text { No specific classification criteria given, but } \\
\text { state arthritis after a proven or putative } \\
\text { infection of the genital tract considered as } \\
\text { sexually acquired reactive arthritis (SARA), } \\
\text { the arthritis (referred to as 'reactive') being } \\
\text { a sterile inflammation of the synovial } \\
\text { membrane, tendons, and fascia. }\end{array}$ & $\begin{array}{l}\text { No screening tool described, not clear if } \\
\text { used. }\end{array}$ & $\begin{array}{l}\text { Method of full examination not described. } \\
\text { Excluded alternative diagnoses, such as } \\
\text { gonococcal arthritis, gout, rheumatoid } \\
\text { arthritis and septic arthritis, by appropriate } \\
\text { investigations. }\end{array}$ \\
\hline
\end{tabular}


Table 7.3. Risk of bias assessment using RTI Item Bank tool

\begin{tabular}{|c|c|c|c|c|}
\hline Item & $\begin{array}{l}\text { Dimension of } \\
\text { bias }\end{array}$ & Keat et al. 1978 & Rich et al. 1996 & Carter et al. 2013 \\
\hline Is the study design prospective, retrospective, or mixed? & $\begin{array}{l}\text { Selection bias; } \\
\text { performance bias; } \\
\text { detection bias }\end{array}$ & Prospective & Prospective & Prospective \\
\hline Are critical inclusion/exclusion criteria clearly stated? & Selection bias & Partially & Yes & Yes \\
\hline Are the inclusion/exclusion criteria measured using valid and reliable measures? & Information bias & Partially & Partially & Partially \\
\hline Is the intervention or exposure clearly described in enough detail? & Performance bias & Partially & Partially & Partially \\
\hline Are the important outcomes pre-specified by the researchers? & Reporting bias & Partially & Partially & Yes \\
\hline $\begin{array}{l}\text { Are interventions/exposures assessed using valid and reliable measures, } \\
\text { implemented consistently across all study participants? }\end{array}$ & Information bias & Partially & Partially & Partially \\
\hline $\begin{array}{l}\text { Are primary outcomes assessed using valid and reliable measures, implemented } \\
\text { consistently across all study participants? }\end{array}$ & Information bias & $\begin{array}{l}\text { Cannot } \\
\text { determine }\end{array}$ & Yes & Partially \\
\hline $\begin{array}{l}\text { Is the length of time following the intervention/exposure sufficient to support } \\
\text { the conclusions of the study regarding primary outcomes? }\end{array}$ & Attrition bias & $\begin{array}{l}\text { Cannot } \\
\text { determine }\end{array}$ & Partially & Partially \\
\hline Did attrition from any group exceed 20 percent? & Attrition bias & $\begin{array}{l}\text { Cannot } \\
\text { determine }\end{array}$ & Yes & Yes \\
\hline In cases of high loss to follow-up, is the impact assessed? & Attrition bias & $\begin{array}{l}\text { Cannot } \\
\text { determine }\end{array}$ & No & No \\
\hline Are any primary outcomes missing from the results? & Reporting bias & Yes & No & No \\
\hline $\begin{array}{l}\text { Are the statistical methods used to assess the primary benefit outcomes } \\
\text { appropriate to the data? }\end{array}$ & Precision & $\begin{array}{l}\text { Cannot } \\
\text { determine }\end{array}$ & Yes & Yes \\
\hline Is the source of funding identified? & Reporting bias & Yes & Yes & Yes \\
\hline Are results believable taking study limitations into consideration? & Overall quality & No & Partially & Partially \\
\hline Overall judgement on risk of bias & - & High risk of bias & $\begin{array}{l}\text { Medium risk of } \\
\text { bias }\end{array}$ & $\begin{array}{l}\text { Medium risk of } \\
\text { bias }\end{array}$ \\
\hline
\end{tabular}




\subsection{Discussion}

\subsubsection{Summary of main finding}

This systematic review found only three studies, of low to moderate quality, reporting an incidence of SARA of $3.0 \%-8.1 \%$. There were insufficient data to perform a meta-analysis.

To the authors' knowledge, this is the first systematic literature review of studies that have assessed the incidence of SARA. It highlights the paucity of data examining the incidence of SARA, which is incongruous since retrospective studies suggest that $C$. trachomatis is the most common trigger of ReA (Kvien et al., 1994, Ozgul et al., 2006) as well as being a common infection (Adams et al., 2004, World Health Organization, 2012b).

Although the small number of relevant published studies means the findings of this review need to be interpreted with caution, the incidence of ReA in the included studies is of concern. As chlamydia infection is asymptomatic in approximately $70 \%$ of females and $25 \%$ of men (Heymann, 2008), SARA should be considered in all people presenting with inflammatory arthritis who are at risk for STIs. In two of the included studies $67 \%$ to $78 \%$ of patients who developed ReA did so after an asymptomatic infection. In addition to the diagnostic consideration for arthritis, an STI screen should be considered for all patients presenting with an inflammatory arthritis that could possibly be ReA, so that any genital infection can be detected and treated. There is some data which suggest this is not common practice (Pease et al., 2013). It seems possible that SARA may be overlooked due to the absence of testing, or the lack of diagnostic criteria available to medical practitioners, not the absence of infection.

The included studies also challenge the accepted view that $\operatorname{ReA}$ after $C$. trachomatis is more common in men, as both of the mixed-sex studies found a similar incidence of ReA in men and women (Rich et al., 1996, Carter et al., 2013). This contrasts population-based and retrospective studies of ReA diagnosed in routine clinical practice, where ReA cases are predominantly males (Hamdulay et al., 2006, Collantes et al., 2007). It is possible that ReA is underdiagnosed in women as genital infections are more likely to be asymptomatic (Heymann, 2008). 


\subsubsection{Strengths and limitations}

This review's strengths include the systematic approach, independent data search and analysis. Limitations include the lack of relevant studies, the medium to high risk of bias assessed to be present within included studies, and the methodological heterogeneity across studies. Additionally, a number of potentially relevant publications were not in English ( $\mathrm{n}=$ 9) and resources were not available to translate these.

Only three studies met the inclusion criteria of assessing the incidence of SARA (Keat et al., 1978, Rich et al., 1996, Carter et al., 2013). Furthermore, Keat et al. (1978) stated that their study was not specifically designed to provide epidemiological data on the incidence of $\operatorname{ReA}$ in the general population or in hospital-based patients with non-specific urethritis. Although the reasons for this statement are unclear, and caution should be applied to the study's interpretation, the reported incidence of SARA of $3.01 \%$ is similar to the incidence reported by Rich et al. (1996) (4.1\%).

The studies had medium to high risk of bias, meaning results should be interpreted with caution. Reporting of study methods was inconsistent and significantly lacking in the oldest study. Future research should make use of reporting guidelines such as STROBE (STrengthening the Reporting of OBservational studies in Epidemiology) to ensure all the relevant information is available to contextualise the work (von Elm et al., 2008).

The studies used different methods and criteria to identify ReA cases. This is a wellrecognised problem in rheumatology where diseases often have heterogeneous presentation and rarely have a single clinical, laboratory, pathological or radiological feature as a 'gold standard' for diagnosis and/or classification (Aggarwal et al., 2015). Because of this, classification criteria have been developed for use in clinical research. Classification criteria are applicable to groups and are more specific than diagnostic criteria which are applied to individuals. No universal diagnostic or classification criteria have been established for ReA. Classification schemes proposed in aiding the diagnosis of spondyloarthropathies include the Amor criteria (Amor et al., 1990), the European Spondyloarthropathy Study Group (ESSG) criteria (Dougados et al., 1991), and the Assessment of SpondyloArthritis International Society (ASAS) criteria (Rudwaleit et al., 2011). Once the diagnosis of spondyloarthropathy is made, further clinical history and symptoms allow identification of the specific 
spondyloarthropathy, including ReA. There is continued debate in the literature about the performance and suitability of these various classification criteria (Zeidler and Amor, 2011, Zeidler and Hudson, 2016).

In this review, only one study used classification criteria (ESSG) making comparative evaluation of the studies challenging, which is an ongoing problem in ReA (Pacheco-Tena et al., 1999, Hannu et al., 2006). Epidemiological studies of ReA require standardised, validated classification criteria to provide uniformity for inclusion of study participants. Until these are established, comparability across studies and a full understanding of ReA (including SARA) will not be possible.

\subsubsection{Conclusions and recommendations}

In summary, there is a lack of data regarding the incidence of SARA. This lack of data feeds into a lack of clinical guidelines and thus physician awareness which perpetuates the problem of underdiagnosis. It is likely that SARA often goes undiagnosed and that only severely affected people are recognised; thus, we cannot determine the true burden of disease. Quantification of the incidence rate of SARA will provide a better evidence-base of the natural history of this condition, as well as allowing improved diagnosis and optimisation of treatment. Studies assessing the incidence of ReA after STI are required, using current STI diagnostic tools that are accurate and sensitive. A suggested screening tool, adapted from Townes et al. (2008), for use in such future studies is included (Figure 7.3), from which participants with positive answers can be selected for full rheumatological review. In consideration of the attrition problems reported by the included studies in this report, assessment of ReA at the initial sexual health clinic visit is advised, along with follow-up at six weeks and three months to enable capture of ReA that takes longer to develop. Specific and sensitive classification criteria must be developed and universally adopted for use in epidemiological studies in order for consistent classification to take place and valid conclusions to be drawn. In clinical practice, it is recommended that at the time an STI diagnosis is made, clinicians discuss with patients the possibility of ReA developing and to return for assessment if they experience any relevant symptoms. 


\begin{tabular}{|c|c|c|c|c|}
\hline \multicolumn{2}{|c|}{$\begin{array}{l}\text { Since developing STI symptoms or since having an STI } \\
\text { test, have you experienced pain, swelling or stiffness in } \\
\text { any of your joints? This could be in any joints including } \\
\text { your fingers, wrists, knees, ankles, toes or the spine. }\end{array}$} & Yes & & $\begin{array}{l}\longrightarrow \text { Questionnaire } \\
\text { terminates } \\
\\
\begin{array}{l}\text { Questionnaire } \\
\text { continues }\end{array}\end{array}$ \\
\hline \multicolumn{5}{|c|}{$\begin{array}{l}\text { Since developing STI symptoms or since having an STI test, have you developed } \\
\text { any of the following new symptoms? }\end{array}$} \\
\hline \multirow{2}{*}{$\begin{array}{l}\text { Joint pain or discomfort } \\
\text { Joint swelling or redness }\end{array}$} & No & Yes & $\longrightarrow$ & $\begin{array}{l}\text { When did this begin? } \\
\text { When did this go away? } \\
\text { (provide option for still } \\
\text { experiencing symptoms) }\end{array}$ \\
\hline & No & Yes & $\vec{\longrightarrow}$ & $\begin{array}{l}\text { When did this begin? } \\
\text { When did this go away? } \\
\text { (provide option for still } \\
\text { experiencing symptoms) }\end{array}$ \\
\hline $\begin{array}{l}\text { Morning joint or back stiffness } \\
\text { lasting longer than } 1 \text { hour }\end{array}$ & No & Yes & $\longrightarrow$ & $\begin{array}{l}\text { When did this begin? } \\
\text { When did this go away? } \\
\text { (provide option for still } \\
\text { experiencing symptoms) }\end{array}$ \\
\hline Heel pain & No & Yes & & $\begin{array}{l}\text { When did this begin? } \\
\text { When did this go away? } \\
\text { (provide option for still } \\
\text { experiencing symptoms) }\end{array}$ \\
\hline \multirow[t]{2}{*}{ Lower back pain } & No & Yes & $\longrightarrow$ & $\begin{array}{l}\text { When did this begin? } \\
\text { When did this go away? } \\
\text { (provide option for still } \\
\text { experiencing symptoms) }\end{array}$ \\
\hline & & & & $\begin{array}{l}1 \text { review if period of } \\
\text { possible } \operatorname{ReA}\end{array}$ \\
\hline
\end{tabular}

Figure 7.3. Screening questionnaire suggested for use in studies of the incidence of SARA, adapted from Townes et al. 2008 


\subsection{Chapter summary and next steps}

This chapter aimed to identify an incidence rate for ReA after STI, to aid evaluation of whether it may be an important pathway to STI testing. Given the lack of data regarding the incidence of ReA after STI, valid conclusions about the proportion of individuals this sequela may affect cannot be drawn. However, the findings presented in this chapter suggest that ReA is common after asymptomatic STI and may often go undiagnosed. Therefore, testing those with ReA for STIs may be one method to identify undiagnosed infection. Although the magnitude of disease burden which could potentially be identified through this process is still unknown, it has the potential to prevent onwards transmission of infection (as well as relieve complications for the individual) which could be significant. More research into ReA after STI is required.

This chapter concludes the results section of this thesis. The next chapter will synthesise and discuss the main findings of the studies presented. 


\section{Chapter 8 Discussion and conclusions}

\subsection{Introduction}

STIs are a global public health issue. They are common, have serious negative health consequences, and are preventable. In New Zealand, incidence rates of some STIs, such as chlamydia, are high by international standards (ESR Annual Surveillance Report 2013). Increasing the rates of testing among people at risk of STI is necessary in order to identify and treat existing infections, thereby disrupting onwards transmission. This thesis aims to provide a better understanding of healthcare-seeking behaviour for STI testing, which can be used to inform and design control strategies that will reduce disease prevalence and improve overall population health.

This thesis used a mixed methods approach to investigate aspects of healthcare-seeking behaviour for STI testing. The overall aims of the thesis were to: quantify the time to presentation for STI testing and interim transmission risk in people with STI symptoms; identify factors associated with delayed healthcare-seeking; identify barriers to, and drivers for, seeking an STI test, including a particular focus on STI knowledge; and to systematically review the globally available evidence of the incidence of ReA after STI.

In this final chapter, the main findings of the thesis will be reviewed and discussed in the context of the wider literature, the methodological strengths and limitations will be explored, and the implications for policy, practice and future research will also be discussed.

\subsection{Summary of main findings}

Extensive discussion of results is given in the discussion section of each chapter. The key findings are highlighted in the following section and compared to the international literature. 


\subsubsection{Delay behaviour is common and poses a significant risk of onwards transmission of infection (Chapter 3)}

\section{Key findings:}

- Almost half of new clients at an inner-city SHC had delayed seeking healthcare for more than seven days after the onset of symptoms

- Around a third of people attending an SHC for STI symptoms had continued to have sex after the onset of their symptoms

- Delaying seeking healthcare for more than seven days was associated with having sex while symptomatic

- Infrequent condom use was common among people who had sex while symptomatic with their existing partner

Information about the healthcare-seeking behaviour of New Zealanders for general STI symptoms, and the associated risk of onwards transmission, has not been available before. This thesis presents data that suggests delay behaviour for STI symptoms is common and presents a significant transmission risk.

Many people with symptoms suggestive of an STI (41.7\%) waited longer than seven days to seek healthcare. The proportion of people delaying seeking care for STI symptoms was comparable to results from overseas research (Moses et al., 1994, Hook et al., 1997, Mercer et al., 2007). There were no statistically significant predictors of delayed healthcare-seeking, though this is likely due to the small sample size resulting in this analysis being underpowered. A similar study from the UK also found no predictors of delay (Mercer et al., 2007), although other studies have found various associations including age and gender (Leenaars, 1993, Irwin et al., 1997, Voeten et al., 2004). The only other study in New Zealand to look at delay behaviour focussed on individuals with genital warts and did not examine demographic predictors of delay, therefore there are no other domestic data to compare these results with. To date, this study provides our best understanding of STI healthcare-seeking delay behaviour in New Zealand. These data are critical when considering and designing control strategies to promote testing and other behaviours. 
The study described in Chapter 3 also found that engaging in sexual intercourse after the onset of symptoms was common (30.6\% of participants). This represents a significant opportunity for onwards transmission of infection, although condom use was generally good among those who were having sex with new partners. STI prevention tends to focus on casual sexual partners; however these findings suggest that transmission between regular partners may have been underestimated. People that delayed seeking healthcare for STI symptoms were more likely to have sex while symptomatic; making delayers a potential target group in terms of public health messages regarding the importance of abstinence from sex after the onset of STI symptoms.

\subsubsection{Barriers may arise from individual beliefs and attitudes, from the healthcare system or providers, or from the social environment (Chapter 4)}

\section{Key findings:}

- There were many personal barriers to STI testing, including underestimating risk, perceiving STIs as not being serious, fear of the testing procedure, embarrassment about physical examination, and being too busy

- Structural barriers to testing were also apparent including the financial cost of testing for the individual, and the clinician attributes and attitude

- Stigma plays a significant role in preventing or delaying STI testing, therefore normalising STIs may help to encourage STI testing

This thesis reports on several barriers which were found to delay or prevent healthcareseeking for STI testing in New Zealand. One such barrier was individuals underestimating their STI risk, which has been shown to be common in many other countries (Ethier et al., 2003, Abel and Brunton, 2005, Zakher and Kang, 2008, Fagan and McDonell, 2010, Wolfers et al., 2011, Oliver de Visser and O'Neill, 2013). Other personal barriers included the misplaced assumption that most STIs are not serious, being afraid of the STI test itself, bodily embarrassment, or being too busy to go for a test. These results imply that making people aware of their own risk and the severity of STIs may be one way to encourage STI testing. Further evidence for this comes from a comprehensive study of 275 sexually active 
people in Brighton, UK, in which greater perceived susceptibility and severity were among factors associated with having had an STI test in the last year (Oliver de Visser and O'Neill, 2013). In addition, perceiving STIs not to be serious was associated with healthcare-seeking delay behaviour in a South African study by Meyer-Weitz et al. (2000). However, there has been some suggestion that increased perceived severity of STIs might cause the opposite behaviour for some people, for example if they are afraid of a positive diagnosis (Cunningham et al., 2005). Combining information on the severity of consequences with details on treatment and management of STIs may help to avoid this.

Another significant barrier to STI testing is the stigma surrounding STIs and STI testing. STIs are viewed as 'socially challenging infections' due to the negative effect they have on how a person is perceived by others. This is because being diagnosed with an STI suggests a violation of social norms and values, specifically that an individual has chosen to engage in behaviour often viewed as immoral, such as unprotected sex, sex with multiple partners, or sex with disreputable partners (Gilmore and Somerville, 1994, Lichtenstein, 2003). Stigma negatively affects healthcare-seeking behaviour for STI testing because even just seeking an STI test suggests deviant behaviour has occurred (Lichtenstein, 2003, Foster and Byers, 2008, Hood and Friedman, 2011, Jackson and Roberts, 2015) and this presents a threat to peoples' social identities (Balfe et al., 2010b). What influences testing behaviour is usually perceived stigma (the individual's own perception of whether they may be stigmatised) rather than acted stigma (Theunissen et al., 2015), as was the case in this study, and previous research has shown that individuals who score higher on measures of STI-related stigma are less likely to have had an STI test (Fortenberry et al., 2002, Rusch et al., 2008, Cunningham et al., 2009, Morris et al., 2014).

Fear of stigmatisation seemed to be an important factor delaying seeking STI testing for the participants who took part in the interview study (Chapter 4), and male participants were especially concerned about being stigmatised if they were seen to be requesting an STI test. The results presented in this thesis therefore support the concept that STIs need to be destigmatised and normalised in order to increase access to healthcare (Foley and Patel, 2001). However, as recently noted by Saxton et al. (2015b), normalising must be done with care in order to avoid diminishing the threat that STIs pose, so that individuals are still motivated to 
engage with primary and secondary prevention measures. Evidence that this is a real risk to be aware of comes from a qualitative study from the UK, which reported that reassuring sexual health education interventions appeared to be related to perceptions of low severity of STIs (Darroch et al., 2003). In addition, normalising should be careful not to subordinate the emotional aspects of STIs, as this could then cause the normalising messages to appear too distant from people's experiences in relation to STIs and STI testing, causing them to discount and disregard the messages (Cook, 2013).

Structural barriers were also identified, specifically the financial cost of testing for individuals and clinician attributes and attitudes. However, there appeared to be some flexibility with regards to these barriers. For example, although many people said they would prefer to see a clinician of the same gender, they would still accept an appointment with a clinician of different gender if it was the only one available. With regards to cost, the findings of this research showed people tried to minimise costs by attending healthcare providers that offered free testing (FPCs, the Student Health Clinic), but would pay for testing if they had significant concerns, for example if they were experiencing symptoms. The idea that structural issues are secondary to other concerns such as stigma has also been noted in an Irish study by Balfe et al. (2010a).

Having completed this work, the results were presented to the SHS where participants had originally been drawn from. As a direct result of this research, the SHS has decided to implement strategies to overcome some of the barriers identified. At the time of writing, the SHS is setting up STI self-sampling stations in bathrooms on the University campus which will provide free STI testing to all students in a private environment. 


\subsubsection{Drivers for testing include crisis-events, partner-encouragement, being offered screening, routine healthcare, and having knowledge about STIs (Chapter 5)}

\section{Key findings:}

- STI testing was considered a normal and routine part of health maintenance by some people, this seemed to be especially true for those with a health-centered upbringing

- Having sought a test in reaction to a crisis event, such as developing symptoms, several people described engaging in more regular STI testing thereafter

- Partner-driven testing appeared to be gendered (females encouraging males to be tested) and persuasive

- Opportunistic screening by a GP was acceptable. Of the interviewees in this study, only females had been offered screening by a clinician

- Having a good level of STI knowledge was suggested to be a key driver for STI testing by participants, and appeared to influence regular testing

- Many people felt that the sexuality education they had received at school had been lacking in regards to accurate and representative information about STIs

The opinion that STI testing is a normal part of maintaining health was expressed by some of the interviewees. Many individuals who routinely tested also described their family as being able to openly discuss STIs and/or having parents who were healthcare-professionals, indicating a health-centred upbringing could encourage regular healthcare-seeking behaviour. As expected, crisis events, such as a sexual partner being diagnosed with an STI, were drivers of STI testing. A novel finding from this study was that female prompting of males to get checked was more persuasive than encouragement from male peers, especially if the potential for sex was involved. It also appeared from discussions with interviewees that having an STI test had a positive effect on subsequent healthcare-seeking behaviour for STI testing, which was also demonstrated in a survey of young people in England (Hartney et al., 2015).

Some of the participants in this study had an STI test because it was offered to them by their GP, although this only appeared relevant for a small number of females. A study by Morgan 
et al. (2010) provided some insight into the proportion of STI tests in New Zealand which are clinician-driven; of 415 diagnosed chlamydia cases in the Waikato region, 18\% of the STI tests among females and $6.2 \%$ of the STI tests among males had been offered by the provider. This substantiates the impression from interviews in this study that opportunistic screening is more common among women.

STI knowledge seemed particularly influential in terms of intersecting with the other drivers, most prominently in relation to routine-driven testing where better knowledge appeared to encourage more regular testing. Lack of knowledge has been identified as a barrier to STI testing in many other studies (Tilson et al., 2004, Chaudhary et al., 2008, Balfe et al., 2012, Yeung et al., 2015). Discussions with participants in this study suggested knowledge of the commonality, asymptomatic nature and long-term health outcomes of STIs would encourage testing.

There was a general opinion among the interviewees that sexuality education received at school and elsewhere had not adequately prepared them for the dangers associated with engaging in sexual activity. This reflects the findings of Natsal 2 in the UK, where the majority of men and women across all age groups felt that they ought to have known more about sexual health (including STIs) when they first felt ready to have some sexual experience (Macdowall et al., 2015). The United Nations Educational, Scientific and Cultural Organization (UNESCO) has also recognised this gap, stating in their 2009 International Technical Guidance on Sexuality Education that "far too few young people are receiving adequate preparation which leaves them vulnerable to coercion, abuse, exploitation, unintended pregnancy and sexually transmitted infections, including HIV" (UNESCO, 2009). The findings of this thesis support that judgement, and call for an improvement of sexuality education in New Zealand, including more information about STIs, a move away from scare tactics, and continuation of education into older ages. 


\subsubsection{STI knowledge is associated with previous STI testing (Chapter 6)}

\section{Key findings:}

- STI knowledge questions that were answered poorly were related to treatment, sequelae and the asymptomatic nature of STIs

- STI knowledge was not associated with sexual behaviour after controlling for covariates

- STI knowledge was associated with previous STI testing, where those with a higher level of knowledge were, on average, more likely to have had an STI test in the past

- STI campaign awareness was associated with STI testing, although there was some mediation of this relationship through STI knowledge and sexual behaviour variables

- Individuals were able to self-assess their own STI knowledge level accurately

STI knowledge questions that were answered poorly were related to treatment, sequelae and the asymptomatic nature of STIs. This is important, as knowledge of these factors has implications for encouraging testing. For example, a person who is not aware that STIs often cause mild or no symptoms may assume that if they are not experiencing any symptoms, they are not infected, and so may not seek STI testing.

A relationship between STI knowledge level and previous STI testing was demonstrated, where those with a higher level of knowledge were, on average, more likely to have had an STI test in the past. This association has also been demonstrated in studies from Australia, the UK and Canada (Greaves et al., 2009, Pedrana et al., 2012, Oliver de Visser and O'Neill, 2013, Cragg et al., 2016), although not all studies have found an association (Langille et al., 2009). This thesis contributes to existing knowledge by strengthening the evidence for an association between STI knowledge and testing. However, it cannot imply causality due to the cross-sectional design of the study.

The study presented in Chapter 6 is the first assessment of general STI knowledge in relation to any STI testing conducted among a New Zealand population, although there are some parallels to be drawn from the New Zealand HIV literature. In a study of young gay and 
bisexual men, those who knew that HIV cannot pass through an undamaged latex condom were more likely to have had a HIV test in the past year than those who did not know or were unsure (Lachowsky et al., 2014).

STI knowledge was not associated with sexual behaviour after controlling for covariates, indicating that increased knowledge does not lead to individuals adjusting their risk of STI downwards via increased condom use or reducing the number of sexual partners. This agrees with the existing literature which has not reported lower risk behaviour among those with higher levels of knowledge (Sekirime et al., 2001, Kong et al., 2010, Lazarus et al., 2010, Norbu et al., 2013), and suggests alternative strategies are required. For example, less didactic approaches, which account for the complex social and personal pressures involved in sexual relationships, may be more effective at reducing sexual risk behaviour.

Individuals who responded that they were aware of any STI campaign either now or in the past were more likely to have had a previous STI test than those who were not aware of any campaigns. Adjustment for STI knowledge suggested that this relationship was mediated through those who were aware of campaigns having better STI knowledge, although there was some evidence that a relationship between campaign awareness and STI testing existed after controlling for sexual behaviour characteristics, suggesting a complex relationship. A similar study in Australia assessed STI knowledge and STI testing in relation to awareness of a specific social marketing campaign which aimed to increase healthcare-seeking behaviour and enhance HIV/STI knowledge in gay men (Pedrana et al., 2012). The authors found that men who were aware of the campaign did not have better overall knowledge than men that were unaware, which contradicts the results of the current study. However, in analyses which controlled for STI knowledge and sexual risk behaviour, men who were aware of the campaign were more likely to have had any STI test within the past six months than those unaware. This is very similar to the relationship observed in the data presented in this thesis. 


\subsubsection{The incidence of $\operatorname{ReA}$ after STI is estimated as $3.0 \%-8.1 \%$, but more research is required (Chapter 7)}

\section{Key findings:}

- The incidence of ReA is estimated to be $3.0 \%$ to $8.1 \%$ among those who are diagnosed with an STI

- Only three studies were found to have prospectively assessed the incidence of ReA after STI, and these were of poor to medium quality

- It is important that specific and sensitive classification criteria are developed to enable consistent classification in research studies so that valid conclusions can be drawn

- ReA incidence after STI occurred at a similar rate in both men and women, suggesting that the accepted view that $\mathrm{ReA}$ is more common among men is incorrect

- Many individuals develop ReA after an asymptomatic STI

The systematic review presented in this thesis (Chapter 7) is the first to collate evidence about the incidence of ReA after STI. The review found that only three published studies had prospectively assessed the incidence of ReA after STI. The lack of research is likely due to the methodological challenges presented by this type of study, such as requiring a large sample size and high levels of retention. The incidence rate of ReA after STI is potentially larger than the incidence rate after enteric infection, estimated as $2.86 \%$ to $5.8 \%$ in systematic reviews of this literature (Ajene et al., 2013, Keithlin et al., 2014, Keithlin et al., 2015). More studies are required to address the lack of data regarding the incidence of $\operatorname{Re} A$ after STI.

The review also raised the issue of classification criteria. While a clinical assessment of $\operatorname{ReA}$ is useful on an individual basis, universal classification criteria are needed to consistently classify ReA patients in research studies so that incidence rates may be reliably compared.

The systematic review revealed that ReA incidence after STI occurred at a similar rate in both men and women in the two studies that presented this data (Rich et al., 1996, Carter et al., 2013). This suggests that the accepted notion that ReA is more common in men is incorrect, and may be driven by undiagnosed disease among women; potentially because 
genital infections are more likely to be asymptomatic in women. This finding is important new knowledge for the field of rheumatology, and has the potential to alter the current paradigm about gender differences in ReA. More research is required to confirm this hypothesis.

The review also found that many individuals develop ReA after an asymptomatic STI (67\% to 78\% of participants) (Rich et al., 1996, Carter et al., 2013), which highlights the need to screen ReA patients for STIs even if they do not display any genital symptoms.

\subsection{Synthesis}

This section presents an overall synthesis of the findings from Chapters 3 to 7, combining the quantitative and qualitative results to derive some general conclusions. It aims to weave together the insights gathered from the work of the thesis.

Healthcare-seeking behaviour for STI testing is a complex process, involving numerous factors operating within multiple spheres of human life, and thus cannot be well predicted.

The participants in the qualitative interviews wanted more knowledge about STIs and many said they didn't have good STI knowledge (Chapter 5). The quantitative data presented in Chapter 6 shows that people accurately self-rate their knowledge scores, signifying that young people in New Zealand should be listened to when they say they want better sexual health knowledge.

That said, evidence from this thesis (Chapter 6) suggests that better STI knowledge is not associated with lower sexual risk behaviour. This concurs with the school of thought that knowledge and its rational assessment do not drive behaviour alone, as social and environmental cues and pressures are also influencing behaviour (Kelly and Barker, 2016). There is evidence that better knowledge may be related to STI testing though, which is worth exploring further. A possible reason why knowledge may be related to testing behaviour but not sexual risk behaviour is the social situations these two behaviours exist within. The relative autonomy and longer time-window for enacting testing behaviour compared to 
lowering sexual risk by avoiding sex or using condoms in a given situation, may allow for more opportunity to rationally apply knowledge to this behavioural decision.

Considering the findings of Chapter 3, it seems that even for those who do test, onwards transmission of infection is possible. Therefore prompt/regular testing and abstinence from sex until a negative diagnosis is confirmed, or treatment for an infection has been completed, should be promoted and encouraged.

Combining data from Chapters 4 and 5 about barriers and drivers for STI testing indicates that a successful public health campaign to encourage STI testing in New Zealand should also: a.) make people aware of how common STIs are and their asymptomatic nature, b.) explain the long-term health consequences for both males and females and c.) give detailed explanation of what the STI testing experience will be like, in particular, debunking any myths about it being invasive or painful.

Males were shown to test less than females (Chapters 3 and 6), be more likely to avoid testing due to stigma (Chapter 4), and be less likely to be routine testers (Chapter 5). Therefore, the results of this study indicate that males need to be a particular focus in future STI control activities (see section 8.4.2 for further discussion).

\subsection{Implications of this research}

New Zealand enjoys the small scale that enables coordinated action. As a result, effective change is possible, provided that it is evidence-based. With this in mind, the next section will highlight some implications from this research with regards to improving healthcare-seeking behaviour for STI testing, and ultimately reducing STI incidence.

\subsubsection{Implications for primary healthcare}

An important finding from this research is that barriers to testing are multiple, and delayed healthcare-seeking for STI symptoms is common. A barrier to STI testing which is simple to remove, is the cost of testing. Previous research in New Zealand showed that introducing free sexual health GP consultations for under 25 year olds significantly increased the number of

people attending for an STI test, as well as the number of chlamydia infections diagnosed 
(Morgan and Haar, 2009). Many people in the qualitative arm of the study presented in this thesis felt that they would not test in the absence of symptoms if they were required to pay for the test. Currently in New Zealand, the cost of STI testing for the patient depends on an individual's age and also their location in the country as funding is not consistent across DHBs. Standardising STI testing to be fully-funded for all age groups and providers would likely have a positive impact on STI testing rates.

Opportunistic screening appeared to be acceptable to participants in the qualitative study and an important driver of testing. However, there is evidence from this study and others within New Zealand that it is gender-imbalanced (Paul et al., 2009, Morgan et al., 2010, Azariah et al., 2013). The New Zealand Chlamydia Management Guidelines do not prioritise testing of any gender, although females tend to be viewed as a priority because of their increased risk of sequelae. The emphasis on women, however, overlooks the role of men in the spread of infection and fosters the attitude that STIs are a women's issue and responsibility, which ultimately results in less healthcare-seeking for STI testing by men (Duncan and Hart, 1999). Along with these reasons, new evidence about the role of STIs in male sub-fertility and other long-term consequences advocates for a shift in this attitude (Duncan and Hart, 1999, Abusarah et al., 2013, Mackern-Oberti et al., 2013, Sellami et al., 2014, Woodbridge et al., 2015).

Previous intervention studies in New Zealand which have aimed to offer testing to all sexually active under 25 year olds using criteria recommended in the Chlamydia Management Guidelines (2008), have shown an increase in testing rates (Lawless, 2010, Lawton et al., 2010, Azariah et al., 2013). However, the increases were not sustained after the intervention period in the two studies that measured this (Lawless, 2010, Lawton et al., 2010). A further study also measured the effect of implementing the guidelines in the Waikato region of New Zealand and found no significant increase in testing volume, possibly due to a less intensive intervention or because testing rates of under 25 year olds in this region were already high (Morgan et al., 2012). Although interventions to improve opportunistic screening may be effective at increasing testing and reducing the risk of PID in women at individual level, their impact on rates of infection at a population level appear to 
be negligible and therefore this approach to screening is inadequate to control STI incidence on its own (Low, 2007, Merritt et al., 2007).

In addition to offering STI testing at health visits, taking the opportunity to educate individuals about their risk may also be beneficial. This may require discussing topics which are generally perceived as personal and challenging, even in the context of a GP consultation. Sexual health discussions with GPs have been shown to be infrequent and inadequate in many countries (Verhoeven et al., 2003, Moreira et al., 2005, Fuzzell et al., 2016). A recent study in Auckland showed that only 50.5\% of 3168 gay and bisexual men surveyed believed that their GP knew their sexual orientation or that they had sex with men (Ludlam et al., 2015). Importantly, the men whose GP was aware of their sexual orientation were more likely to have ever had an HIV test, STI tests, and were twice as likely to have had an STI diagnosed. This validates that these are essential conversations for GPs to have with their patients in order to more accurately assess their risk and provide appropriate sexual healthcare. It also helps the patient to understand their own risk, which is highly relevant as underestimating risk was seen to be a significant barrier to testing in Chapter 4. An assessment of sexual health communications within primary care in New Zealand would be useful in order to explore ways that sexuality discussions could be improved for New Zealanders.

Including sexual communication in every consultation would help normalise these types of discussions for both provider and patient, although structural changes such as increasing consultation times may need to take place to enable this. Other potential strategies that could be adopted by GP practices to promote open and honest sexual health communication could include asking parents of adolescent patients to leave the consulting room to improve confidentiality, and using signage that indicates safe and accepting environments with regards to diversity of sexual behaviour and identity (Fuzzell et al., 2016). Regarding sexual health as a 'personal' issue is one factor which leads to the lack of sexuality discussions in healthcare settings (Maurice, 1999). It is also incompatible with young peoples' everyday environments, with the uninhibited presence of sexuality in the media and the increased access to pornography and dating apps (Beymer et al., 2014, Martyniuk et al., 2016). 
Treating sexual health as a personal matter maintains its status as a secretive and taboo topic, which only results in poorer sexual health outcomes for individuals.

\subsubsection{Implications for intervention design}

It is well established that females seek STI testing more often than males (Geisler et al., 2006, Morgan and Haar, 2008, Morgan and Bell, 2009, Cuffe et al., 2016). The results of this thesis agree; females were more likely to report a previous STI test than males. This advocates for more attention to be paid to male attitudes and behaviours with regards to STI testing, and to develop interventions specifically designed to encourage males to seek testing. Previous literature has identified the role of masculinity as one potential reason for the gender disparity in testing, specifically that males feel invulnerable, or conceal their vulnerability by not seeking healthcare when needed (Davies et al., 2000, Shoveller et al., 2010, Balfe et al., 2012). Other studies have shown that men perceive STIs to be 'women's diseases' (Mills et al., 2006, Chaudhary et al., 2008). Results from this thesis suggest that stigma-response may also play a role; while females are more commonly stigmatised with regards to STIs (Hyde et al., 2008, Senior et al., 2014, Jackson and Roberts, 2015), the females in the qualitative study described actively rejecting this stigma and testing anyway, whereas males were more fearful of being labelled and avoided testing. Of concern, evidence from New Zealand shows that men with multiple partners in the past year (more at risk of STIs) were less likely to have a GP than men with one or no partners (Dickson et al., 1998), although this study was conducted in 1993-1994 and may no longer be representative. With this in mind, off-site testing may be effective for males who don't attend healthcareproviders, perhaps accessed through sports clubs (Kong et al., 2009) or home-testing (Stephenson et al., 2000).

As STI knowledge was associated with previous STI testing behaviour, improving knowledge among those at risk of STIs may be one way to increase testing rates. Previous intervention studies from overseas have attempted to improve knowledge through means such as educational programmes or sending SMS messages, and have measured subsequent STI testing in the intervention group (Tyden et al., 1994, Tyden et al., 1998, McClean et al., 2000, Lim et al., 2012). These studies have had mixed results. The smaller scale of New Zealand compared to many other developed countries affects how interventions work 
compared with other countries. Therefore, New Zealand needs local data, and international studies need to be repeated so that the local efficacy of interventions can be evaluated.

National or local sexual health campaigns have also been utilised to encourage STI testing in other countries. These may be designed to impart STI knowledge, or alternatively may aim to normalise conversations about STIs and STI testing. Interviewees in the study presented in Chapters 4 and 5 felt that these types of campaigns would be effective. Currently in New Zealand, campaigns involving messages to encourage STI testing are limited to online sites such as the 'Just The Facts' website (http://www.justthefacts.co.nz/). While these initiatives are extremely useful, especially when the target population is a generation with high levels of internet use, a widespread multi-media campaign may also be beneficial. This has been observed with regards to primary prevention among gay and bisexual men in Auckland; seeing condoms promoted in multiple ways was predictive of frequent condom use with casual partners (Saxton et al., 2015a). Similarly, a multi-media campaign in England called 'Chlamydia. Worth talking about' was associated with an increase in positive chlamydia tests, due to increased testing of high risk individuals and groups in the target population who were previously less likely to come forward for testing (Gobin et al., 2013). However, the campaign appeared to have differential effects on coverage in various socio-demographic groups. Any campaign would need to be carefully designed so that it would not detract from condom promotion activities (Saxton et al., 2015b) and would be relevant to all sections of the target population (Gobin et al., 2013).

A more holistic approach which goes beyond solely improving knowledge and uses a combination of approaches at multiple levels of influence may be effective (Auerbach and Coates, 2000, DiClemente et al., 2007). The most successful mix will vary depending on local conditions, so New Zealand-based research is required in order to sharpen implementation of interventions. The field of social diagnosis can provide important insights into what will need to be included, as it connects the process of seeking a diagnosis to cultural, social, political and economic conditions or factors (Brown et al., 2011, Jutel, 2015).

\subsubsection{Implications for sexuality education}

An important implication of this thesis is the need to improve sexuality education in New Zealand. Sexuality education is important to young people, as evidenced in this work. 
However, conservative views of others have led to a less than optimal approach to sexuality education in New Zealand schools, including the proviso that schools are free to decide what content is taught (Ministry of Education, 2015). This has led to incomplete and inconsistent sexuality education in New Zealand. One commonly used argument against comprehensive sexuality education in schools is that it will encourage young people to engage in more sexual activity and at a younger age. International research has consistently invalidated this claim (Ellis and Grey, 2004, Kirby et al., 2007).

The participants in the study presented in Chapter 5 felt that the education they received was designed to scare rather than to inform. Employing stigmatising messages and images to motivate people to adopt risk-reduction behaviours is a common feature of sexuality education programmes (Hood and Friedman, 2011). Presenting STIs as a result of 'bad' and 'irresponsible' behaviour ignores the broader sociocultural determinants that influence sexual partnering, risk and STI acquisition. It also re-enforces negative perceptions of those who acquire STIs, which ultimately leads to the 'othering' of STIs. This does not encourage healthcare-seeking behaviour, as people then deem STIs to happen to 'bad' people and consequently do not feel at risk themselves.

There is evidence that sexuality education is more likely to be effective if it responds to what young people think, believe, and need (Ellis and Grey, 2004). Therefore young people should be taken seriously when requesting a departure from scare tactics and the use of a more open and honest approach to sexuality education. Moving away from a danger prevention focus may be one way to facilitate this (Allen, 2007). It is also important to consider other ways in which sexuality education programmes may be effective (Ingham, 2005), for example, in increasing self-confidence with regards to sexual issues, aiding identification of unhealthy relationships, or improving knowledge about the logistics of sexual activity. These aspects are also key to enable young people to have fulfilling and coercive-free sex lives.

Sexuality education does not end at the classroom door however. The interviews conducted as part of this work indicated that talking about sexual health openly in the family home could lead to more regular STI testing behaviour as a young adult. Guidance on how to talk to children and teenagers about sexual health in a positive and non-stigmatised way may be beneficial for parents. 


\subsubsection{Implications regarding $\operatorname{Re} A$}

Other implications stemming from the results presented in this thesis include recommendations with regards to the management of ReA in clinical practice. With regards to identifying undiagnosed infection, it is important that rheumatologists request an STI test for patients presenting with ReA if a triggering organism has not already been identified. In addition, given the potential number of people developing ReA after STI (3.0\% to $8.1 \%)$ it may be advisable for clinicians in primary care to discuss the possibility of ReA developing at the time of STI diagnosis, and to encourage patients to return if they experience any relevant symptoms.

\subsubsection{Looking ahead}

Mathematical modelling has suggested that annual screening of 30\% to $40 \%$ of all people under 25 years is required to decrease chlamydia prevalence (Regan et al., 2008). Although STI testing coverage is generally good in New Zealand, it is a way off these levels (ESR Annual Surveillance Report 2014). Achieving and then sustaining high levels of healthcareseeking behaviour will be challenging. Therefore, alternative approaches to controlling STIs also need to be fully supported, including condom promotion and vaccine development. Vaccines have been demonstrated to have a considerable impact on two STIs so far; Hepatitis B and, more recently, HPV (Harper et al., 2004, Harper et al., 2006, Cutts et al., 2007), and are more consistently effective at preventing infection than other types of interventions (Wetmore et al., 2010). Development of a vaccine for HIV has been a subject of international focus for some time (Girard et al., 2011), and there is also considerable research being conducted into chlamydia and gonorrhoea vaccination (Christodoulides, 2014, Hafner et al., 2014). While we await breakthroughs in this technology, primary prevention (condoms) and secondary prevention (test and treat) methods need to be optimised and encouraged. 


\subsection{Strengths and limitations}

\subsubsection{Strengths}

The studies described in this thesis have a number of strengths; these are discussed in turn below.

\subsubsection{Use of a mixed method approach}

This thesis used a multistage, multilevel mixed methods framework (Figure 1.1) to understand and describe healthcare-seeking behaviour for STI testing in more detail. Integration of quantitative and qualitative data took place at the study design level, methods level and the integration and reporting level (Fetters et al., 2013, Creswell, 2015). The study design took the form of a three-phase sequential approach, with focus group qualitative work being conducted first (Appendix B), followed by quantitative data collection, followed by qualitative interviews (Figure 1.1).

Methods integration was achieved by 'connecting', where the interview participants were selected from the sample of participants who took part in the questionnaire, and by 'building', where the results of the focus groups directly informed the recruitment protocol for the quantitative data collection (Fetters et al., 2013). In addition, the quantitative results about delay behaviour and reasons for testing informed data collection about barriers and drivers for healthcare-seeking within the qualitative interview phase of the study. Integration at the interpretation and reporting level is narrative, with data from both methods being used to inform the conclusions of this research. Using a mixed methods approach to this research has allowed for a deeper and more holistic assessment of the topic and the potential implications.

\subsubsection{Sampling}

Patients visiting specialised SHCs and those visiting GPs for testing tend to have different demographic characteristics and risk behaviours (Llewellyn et al., 2012, Mercer et al., 2013a). Therefore, using data from both settings provides an opportunity to look at this across a range of risk profiles. Both groups were chosen because they are at high-risk of STIs and were therefore epidemiologically important groups. 


\subsubsection{Response rate}

The higher the response rate, the less risk that results will be affected by non-participation bias and the more representative they will be (Fenton et al., 2001a, Carey et al., 2008). The response rate for the questionnaire study at the SHC (Chapters 3 and 6) was high at $73 \%$. The qualitative interview study (Chapters 4 and 5) recruited an initial 30 out of 57 invited participants before data saturation was reached at 24 interviews, also representing a good response rate $(53 \%)$ for qualitative research. Unfortunately a response rate could not be calculated for the SHS sample in Chapter 6, as participation was self-select. This limits the ability to interpret whether that particular sample is representative. Comparison of demographics indicated that the age and ethnicity profile of the study sample was similar to the overall SHS population, but that there was a slightly higher proportion of females in the study group.

\subsubsection{Defining a finite period for the number of sexual partners}

It is well accepted that asking about the number of sexual partners in a specified period of time provides more reliable data than asking about the lifetime number of partners, due to issues of participant recall. With regards to the optimum time period to specify, there is not a consensus (Schroder et al., 2003, Jaccard et al., 2004). In general, longer intervals result in more inaccurate recall of sexual partners because a more elaborate reconstruction of events is required rather than a simple scanning of more recent events (Fenton et al., 2001a). A period of 12 months was chosen for the questionnaire used in this thesis as it was felt to be long enough to provide an accurate representation of an individual's risk behaviour but not too long that it might affect participants' ability to accurately remember this information. This period is commonly used in behavioural research in relation to STIs (Catchpole et al., 1997, Hughes et al., 2000, Psutka et al., 2012, Connor et al., 2015).

\subsubsection{Knowledge questions}

The knowledge tool used in the study was shown to be strongly correlated with a comprehensive, validated STI knowledge assessment tool (Jaworski and Carey, 2007), suggesting it was measuring a similar construct to the comprehensive measure. 


\subsubsection{Infection coverage}

Most studies which have examined healthcare-seeking for STI testing have done so on a disease specific basis, most commonly focussing on chlamydia (Rose et al., 2008, Balfe et al., 2010a, Richardson et al., 2010, Balfe et al., 2012, Hartney et al., 2015, Theunissen et al., 2015). This research examines testing across various types of STIs, enabling the research findings to be more widely applied.

\subsubsection{Limitations}

The limitations of the studies presented in this thesis are discussed below.

\subsubsection{Sample size}

Due to resources and time limitations, the number of participants involved in some of the studies was small. It is possible that, in the studies described in Chapters 3 and 6, some of the analyses may have been underpowered. In addition, more participants would have allowed for further stratification within analyses, for example, to look at the effect of gender on the likelihood of engaging in sex while symptomatic. Ideally, future studies examining healthcare-seeking behaviour for STI testing should be larger to provide statistical power for more in-depth analyses.

\subsubsection{Generalisability}

The findings of these studies should not be generalised to other groups. People attending SHCs are likely to differ in their demographics, sexual behaviour, STI rates and testing behaviour to the general population (Johnson et al., 1996, Gerver et al., 2011). There was also a high proportion of people with a tertiary education in the SHC sample in this study, which has been observed elsewhere in relation to research participation (Smith et al., 2003a). Similarly, university students are not representative of all young people. Furthermore, as recruitment took place through the health services of a single institution, the findings may not be relevant to other university populations (de Visser et al., 2005). For example, access to STI testing may be different at other universities within New Zealand, and will certainly be different to that available to the broader population of a similar age.

In addition, the studies were all conducted in one geographical area of New Zealand, limiting the extent to which the results can be generalised to other parts of the country. It is possible that individuals living in rural areas demonstrate different healthcare-seeking behaviour due 
to accessibility of services (Spleen et al., 2014), have different levels of STI knowledge, and also experience different motivators and barriers to testing than those living in a large city.

\subsubsection{Social desirability bias}

The majority of data in this thesis have been acquired through self-report, either in questionnaires or interviews. The exceptions were STI diagnoses for the current visit and data used in Chapter 7 which involved rheumatological assessment. Self-reported data is prone to social desirability bias (respondents' unwillingness to report sensitive and socially censured attitudes or behaviours). Unfortunately it is usually impossible to verify reports of sexual behaviour and healthcare-seeking behaviour. Approaches taken to minimise social desirability bias in this study included using anonymous, self-completed questionnaires and ensuring the interviews were conducted in a neutral, non-judgemental manner.

\subsubsection{Incentivising bias}

The use of incentives has been shown to improve participation in research, including in the field of sexual health, and can therefore reduce non-response bias and increase power (Church, 1993, Deren et al., 1994, Kamb et al., 1998). However, it is possible that only a certain type of non-respondent is encouraged to participate when incentives are utilised, resulting in participation bias (Galea and Tracy, 2007). This presents a serious dilemma for the researcher in balancing potential biases. Specific to this research, results of focus groups (Appendix B) indicated that incentives would benefit recruitment. Given the relatively short time frame for recruitment, it was decided that small incentives should be used for both quantitative and qualitative arms of the study (entry into a prize draw for questionnaire participants and a small-value grocery voucher for interview participants).

\subsubsection{Cross-sectional study design}

The cross-sectional study design makes it difficult to attribute causation, particularly in the study assessing the association of STI knowledge with STI testing behaviour (Chapter 6). Although a clear relationship between knowledge and testing was observed, it is not possible to untangle whether having a higher knowledge level prompted testing or whether experiencing an STI test led to a higher level of knowledge. An RCT or prospective study is required to be able to more confidently attribute the direction of effect. 


\subsubsection{Accurate assessment of STI knowledge}

Given that the questionnaire was not administered in a monitored environment, it is possible that people could have used the internet to look up the correct answers to the STI knowledge questions, an inherent risk of self-complete knowledge tools (Sherman et al., 2016). This is more likely to have occurred for those who completed the questionnaire online rather than in the clinic setting waiting rooms. However, subset analysis indicated that those who completed the survey online did not have higher scores than those who completed the study in the waiting room environment. The knowledge assessment questions all included an 'I don't know' option so that participants weren't forced to guess an answer which would not accurately represent their knowledge level.

\subsubsection{Missing data}

Missing data may be random or may indicate item response bias in which respondents who do not answer a particular question are systematically more or less likely to have demonstrated the particular behaviour. In the latter situation, results may be skewed towards a particular behaviour or outcome which is not an accurate representation of the sample. Item non-response was low for all questionnaire variables, although formal analysis of missing data patterns was not undertaken. There are many approaches to dealing with missing data, which have been discussed and evaluated in detail elsewhere (Little et al., 2012, Dong and Peng, 2013, Bannon, 2015). For the analyses presented in this thesis, a complete case analysis approach was chosen. Most analyses include only a few variables meaning the number of cases removed due to missing data would have been small. However, in analyses which involved multiple variables such as logistic regression analyses adjusted for demographic and sexual behaviour variables, the larger number of variables will have resulted in the removal of more cases and may have had some influence on the results.

\subsubsection{Unmeasured variables}

With regards to the quantitative studies (Chapters 3 and 6), questionnaire space was limited, meaning exploration of the potential effects of some additional factors on outcomes was not possible. Some examples of potential influencing factors include socioeconomic status, concurrency of sexual partnerships, sexual risk perception, and attitudes towards STIs. One benefit of using qualitative methods in this thesis was that some of these could be explored in 
detail within a small group of respondents. For example, sexual risk perception and attitudes towards STIs were observed in the qualitative data.

Due to questionnaire space, it was also not possible to include event-level data collection (e.g. questions about the last few sexual events individually). Therefore, condom use was asked about in relation to usual practice, and so assumes that sexual risk behaviour is stable over time and with different partners. Collapsing data collection in this way across multiple partners may be ignoring important variability (Mustanski et al., 2014).

The type and severity of symptoms was also not measured in the quantitative studies. As symptom type or severity are likely to influence time to presentation (Myers, 2011), they may be potential attributes relevant to the analyses presented in Chapter 3.

\subsubsection{Never-testers}

The qualitative studies (Chapters 4 and 5) restricted participants to those who had had an STI test in the previous 18 months. While this inclusion criterion was chosen to elicit true drivers for testing, it presents a problem when interpreting barriers to testing. Many participants spoke of factors that had delayed them from seeking testing, but eventually all had sought or been offered a test. It is possible that the barriers to testing would be different for those who had never had a test.

Similarly, Chapter 3 described assessing delay behaviour among people experiencing STI symptoms. Although these people may have been classed as 'non-attenders' before accessing healthcare, they had all sought healthcare eventually, by definition of inclusion in the study. It may be that there are individuals who would never seek healthcare and they may have different demographic characteristics and sexual behaviours to those who do, which may impact on STI transmission to new individuals. Therefore, this would be an important group of people to identify and include in future research around this topic.

\subsubsection{Investigator biases}

Investigator bias is inherent and unavoidable in any research project, and the involvement of the researcher cannot be overlooked. The two disciplines of scientific enquiry represented in this thesis require two different approaches. Quantitative research reveres a removed approach where a key consideration is to influence the data as little as possible, while 
qualitative research requires complete immersion in the data and thus influence is unavoidable as the researcher becomes part of the data (Green, 2009). It is inevitable that the researcher's own worldview will have shaped some of the data in this thesis, certainly that obtained by qualitative methods. This will have occurred through questions asked during interviews, the way they were asked, or even the way the data were analysed and interpreted. In addition, how people viewed the researcher may also have biased results. For example, while talking about sensitive issues related to sexual health, female interviewees may have responded to the researcher in a different way than male interviewees, and perhaps in a different way again to how they may have responded to a male interviewer. However, Bellamy et al. (2011) have argued that while difference in gender may have an impact on the research interview, it is not the case that 'matching' participants with the researcher will result in the production of more authentic research accounts. Participants were offered the opportunity to be interviewed by a male researcher, but none of the participants (of any gender) took up this option.

The limitations presented above do not mean that this study cannot make an important contribution to the field. There has been little research assessing delay behaviour for STI symptoms over the past decade, and what little has been done has predominantly been in Europe and North America (Table 2.3). In addition, there is not much information about the drivers for STI testing available, as most studies have focussed on barriers to testing. Lastly, STI knowledge has mainly been assessed in relation to sexual risk behaviour or HIV testing, with very little data on its association with more general STI testing. This study contributes new information to the field, using data from a country that has a high incidence of STIs.

\subsection{Recommendations for future research}

Based on the findings of the studies described in this thesis and other relevant literature, some recommendations for future research can be made. 


\subsubsection{Study design considerations}

The results of this work suggest that better STI knowledge may be associated with STI testing behaviour. Only a few studies have assessed this relationship prospectively, and have shown mixed results (Tyden et al., 1994, Tyden et al., 1998, McClean et al., 2000, Lim et al., 2012). None of these studies took place in New Zealand. A large-scale, multi-centre, RCT would provide more conclusive evidence about whether improving STI knowledge results in increased healthcare-seeking behaviour for STI testing.

\subsubsection{Assessment of healthcare-seeking behaviour among specific populations}

Māori and Pacific peoples experience higher rates of STIs compared with other ethnicities (ESR Annual Surveillance Report 2014). The reasons for this are unclear and likely complex, although accessing healthcare may be a potential contributing factor (Ward et al., 2013). The studies presented in this thesis did not oversample Māori and Pacific peoples, and as such, the number of participants identifying as Māori or Pacific peoples is small. This limits the ability to draw any valid conclusions about specific issues with regards to healthcare-seeking behaviour for STI testing for these people. Understanding the specific needs and barriers of Māori and Pacific peoples is necessary to enact evidence-based changes leading to more equitable sexual health within New Zealand.

The studies described in this thesis took place in an urban setting, and cannot be generalised to more rural populations. People that live outside of the main centres in New Zealand have poorer access to sexual healthcare due to a lack of specialist services and there is some evidence from the USA that people who live in rural areas are more likely to avoid healthcare (Spleen et al., 2014). It would therefore be useful to repeat some of this work in a rural population in order to compare and contrast results. This would inform whether different or similar approaches are needed to maximise healthcare-seeking behaviour for STI testing in these different settings.

Lesbian, gay, bisexual, transgender, intersex and questioning (LGBTIQ) people may experience different barriers to healthcare than heterosexual cis-gender individuals as a direct result of their sexual or gender identity (Arbeit et al., 2016, Johnson et al., 2016). Mayer et al. 
(2008) catalogue these barriers into four main types: reluctance to disclose sexual or gender identity when receiving medical care; insufficient numbers of providers competent in dealing with LGBTIQ issues as part of the provision of medical care; structural barriers that impede access to health insurance and limit medical rights for LGBTIQ people; and a lack of culturally appropriate services. Future research should investigate the specific barriers to STI testing for LGBTIQ people in New Zealand, as these are currently unknown.

\subsubsection{Service provider barriers to STI testing}

Policies regarding STI prevention need to be informed by research that explores not only the target populations' attitudes and barriers, but also addresses the 'supply side' of preventive health care, specifically service providers. Given that most STIs are diagnosed in general practice within New Zealand, it would be helpful to know how long provider delay is and what the determinants and consequences of this are.

\subsubsection{The epidemiology of STI testing within rheumatology}

The systematic review presented in Chapter 7 indicates there is a desperate need for prospective studies investigating the incidence of ReA after STI. Results from the existing studies suggest a significant number of individuals with an STI go on to develop ReA (3.0 to $8.1 \%$ ). In addition, many people with ReA had an asymptomatic STI. Therefore, screening ReA patients for STIs could potentially uncover undiagnosed STI, however, it is not clear if this is commonly practised (Pease et al., 2013). A questionnaire-based survey of rheumatologists or a retrospective review of patient notes may shed some light on the proportion of ReA patients with unknown triggering infection that are screened for STIs. Results would inform whether this opportunity to identify undiagnosed infection is being fully utilised, or whether an update to continuing medical education (CME) activities is warranted for clinicians who care for rheumatology patients.

\subsubsection{Treatment acceptance}

This thesis has focussed on the initial stage of secondary prevention methods; how an individual seeks STI testing and the factors involved. However, the premise that encouraging those at risk of STI to seek testing will have a positive effect on STI incidence rates, relies on the assumption that individuals who receive a positive diagnosis return for and comply with 
treatment. A previous study in the Waikato region of New Zealand found that of 415 people diagnosed with chlamydia, 35 people $(8.45 \%)$ failed to attend for treatment or had no documented treatment or outcome (Morgan et al., 2010), and results from overseas studies report even higher rates (Butz et al., 2016). It would be useful to expand on this previous work by assessing the epidemiology of STI treatment nationally, including measures of uptake, timeliness and compliance, to determine if current practice is effective.

\subsection{Conclusions}

STIs such as Chlamydia are common in New Zealand, and have serious associated morbidity. As such, STIs cause significant physical and psychological harm to individuals and impose a substantial financial burden to healthcare systems (Hughes and Lowndes 2014). Reducing the onwards transmission of infection, the focus of secondary prevention methods, will decrease the magnitude of these negative outcomes. One method of secondary prevention is asymptomatic opportunistic screening, however large-scale interventions employing this method have not produced the reduction in population-level incidence rates that many had hoped for, meaning that instead, a focus on encouraging those at risk of STIs to seek testing is required.

Mathematical models indicate that the duration of infection is directly related to the spread of that infection (Macdonald, 1952, Garnett, 2002, Smith et al., 2012). Therefore, if we can reduce the duration of infection, the amount of onwards transmission could be minimised (Ward et al., 1997). The results of this thesis provide additional support for that concept, by showing that delayed healthcare-seeking is associated with having sex while symptomatic. As delay behaviour is potentially contributing to STI transmission, it may be influencing incidence rates and causing a downstream burden on the health system, and thus needs to be addressed as a priority.

This work found no predictors of delay behaviour, which may have been due to statistical power, unmeasured variables, or no differences between people who delay seeking care and people who don't. In reality, the ability to predict behaviour accurately is very difficult. This is because it is influenced by the social, political and economic environment, often 
unmeasured in epidemiological research. This validates the belief that understanding the reasons people do what they do from their own perspective is important, and is why this thesis combined these quantitative results with qualitative work.

The qualitative interviews revealed many barriers to healthcare-seeking, some of which can be addressed quite simply, for example, by reducing the cost of testing and making people more aware of the risk of STIs. Other barriers, such as social stigma, will be harder to overcome. Several drivers for testing were identified as a result of the qualitative interviews: crisis, partners, clinicians, routines and knowledge. This information can be used in the design of future public health campaigns aimed at encouraging people to seek STI testing. Both qualitative and quantitative data from this thesis showed that STI knowledge may be an important factor influencing healthcare-seeking behaviour for STI testing. As STI knowledge has been shown to be modifiable via educational interventions, this has important implications for improving levels of STI testing.

Finally, the results of this research indicate that a holistic approach to encouraging testing is required, including addressing misconceptions, working with healthcare providers to minimise structural barriers, improving and extending school-based sexuality education, improving STI knowledge, and developing initiatives to change social views about STIs. 


\section{Appendix A. Questionnaire and detailed explanation of questionnaire items}

\author{
Page 1: About you \\ Q1 What is your gender? \\ O Male \\ O Female \\ o other \\ Q2 How old are you?

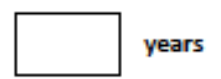 \\ Q3 Which ethnic group do you identify with? \\ O New Zealand European \\ ○ Mãori \\ O Samoan \\ O cook islands Māori \\ O Tongan \\ O Niuean \\ O chinese \\ O Indian \\ O other (please specify) \\ - Would prefer not to answer
}

Q4 What qualification are you studying for now?

O Bachelor Degree

O Bachelor with Honours Degree

- Masters Degree

- Graduate Diploma

O Doctorate

O Other (please specify)

Q5 Do you have any of the following medical conditions? (Please tick all that apply)

- Arthritis or other rheumatic disease

O Asthma

- cancer

- Depression, anxiety or mental health disorders

O Diabetes

O Epilepsy

O Heart disease

- Kidney disease

O Liver disease

O Lung disease

O sleeping disorder

O other (please specify)

\author{
ViSTI Study
}

Q6 Do you smoke?
O No
O Yes

For office use only

Survey ID:

If you smoke cigarettes, please enter how many cigarettes you smoke per day

If you smoke roll ups, please enter how many $50 \mathrm{~g}$ bags of tobacco you use per week

Q7 How often do you have a drink containing alcohol?

O Never

O Less than monthly

- 2-4 times a month

- 2-3 times a week

- 4 or more times a week

Q8 How many standard drinks do you have in a typical day when you are drinking? A $330 \mathrm{ml}$ (small) can of beer is approx. 1 standard drink, a 100ml (small) glass of wine is approx. 1 standard drink
- 1 or 2
O 3 or 4
O 5 or 6
○ 7 to 9
O 10 or more

Q9 How often do you have 6or morestandard drinks on one occasion?

O Never

O Less than monthly

O Monthly

O Weekly

- Daily or almost daily

Q10 Have you ever been sexually active?

O Yes

O No

O Not sure

- Would prefer not to say

Q11 Are you attending Student Health Services today for any of the following reasons?

O STI test - please read and respond to the tick-box question below and then continue to PAGE 2

O Cervical (pap) smear - please read and respond to the tick-box question below and then continue to PAGE 3

O Contraceptive pill check - please read and respond to the tick-box question below and then continue to PAGE 3

If your appointment today includes an STI test, we would like to link your results to your questionnaire. Please tick this box to indicate that you agree that your test results and treatment information can be accessed from Student Health. Your student number will be used for the linking of results and your name will not be recorded, please enter your student number below:

Student number:

If you are not attending for any of the above reasons, please continue to PAGE 4 of this questionnaire 


\section{Page 2: If you are attending Student Health for an STI test, please complete this page}

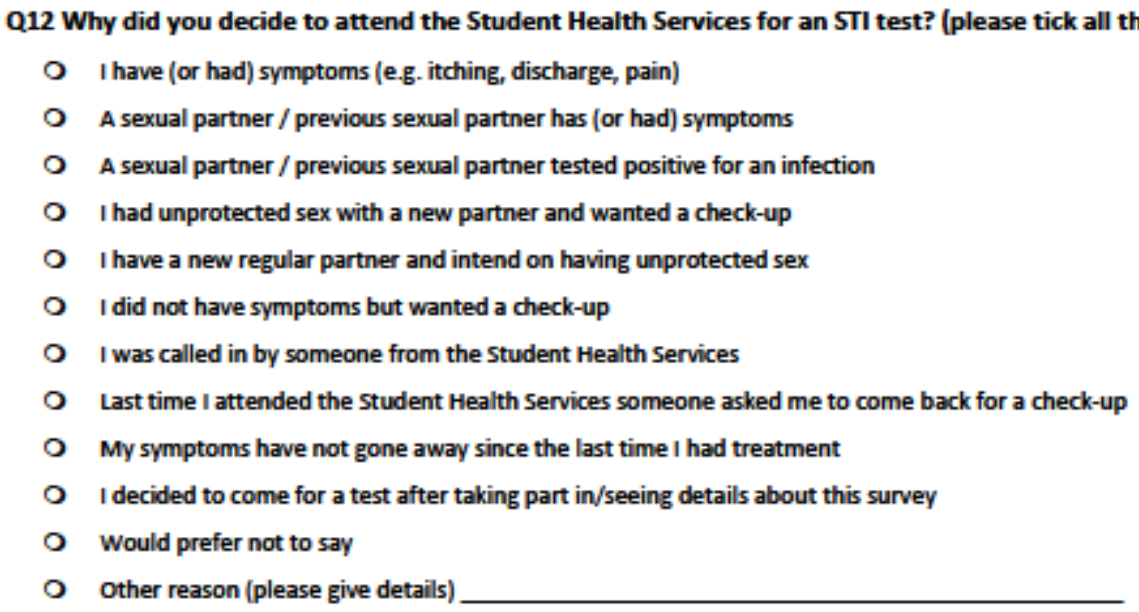

If you have/have had STI symptoms, please complete the following section. If you have NOI experienced STI symptoms, please continue to PAGE 3

Q13 Is this your first visit to any health provider for this set of symptoms?

O Yes

○ No

Q14 How many days were your symptoms present before contacting health services? (this can be any clinic, GP or health professional)

If you contacted the health services on the day the symptoms started, please enter 0

days

Q15 Have you attempted any self-treatment in this time?

- Yes (please give details in space below)

○ No

Q16 Have you had sex since you first thought you might need to go to a clinic or health services?

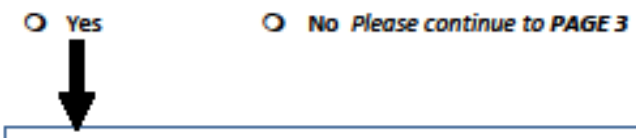

Q17 Since you first thought you might need to go to a clinic or health services, how many partners have you had sex with?

Q18 Since you first thought you might need to go to a clinic or health services, how often have you used condoms when you had sex (with all partners)?

partners

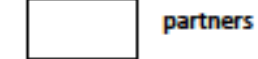

How many of these were new sexual partners? ['new' means since the onset of your symptom

new partners
O None of the time

O some of the time

O Half of the time

O More than half of the time

- All of the time 
Page 3: Sexual health history

Please complete this page if you are attending Student Health for an STI test, cervical smear or pill check. If you are $M O L$ attending for any of these reasons, please skip to PAGE 4.

Q19 Please tick the statement that best applies to your current circumstances

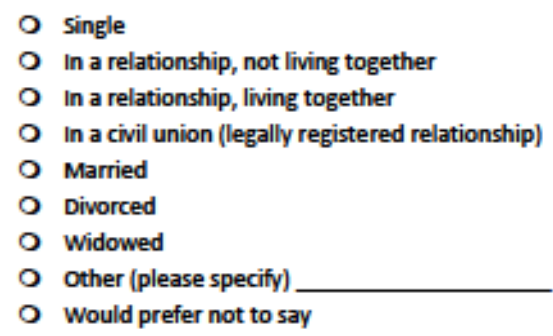

Q20 Do you use condoms?

O None of the time

O some of the time

O Half of the time

O More than half of the time

O All of the time

Q21 What type of contraception (birth control) do you use? (Please tick all that apply)

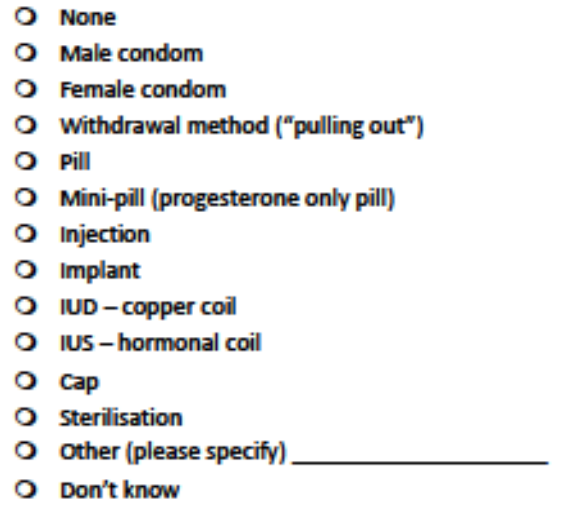

Q22 Before this visit to the Doctor/Nurse at Student Health Services; have you ever had a test for a sexually transmitted infection (STI)?
O Yes
O No
O Not sure
O Would prefer not to say

Q23 Before this visit to the Doctor/Nurse at Student Health Services; have you ever been diagnosed with an STI?

O Yes

O No

O Not sure

Would prefer not to say

If yes, please enter the names of any STIs you have previously been diagnosed with, for example chlamydia, gonorrhoea, herpes, HIV.

Q24 How many sexual partners have you had in the past year? Please enter 0 for no sexual partners. Leave blank if you would prefer not to say.

Number of male sexual partners in the last 12 months

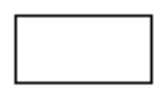

Number of female sexual partners in the last 12 months

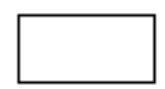

Q25 In the past year, have you had sex with someone you believe had an STI?

O Yes

O No

O Would prefer not to say

If yes to Q25, please enter the names of the $S T 1$ if known, for example chlamydia, gonorrhoea, herpes.

If yes to Q25, did you become aware of this before or after sex with this person?
O Before
- After
- Don't know
- Would prefer not to say

Q26 Have you ever received a Human Papillomavirus (HPV) vaccine?

O Yes

O No

O Not sure

O Would prefer not to say

Q27 Have you ever had an HIV test?

O Yes

○ No

O Not sure

Would prefer not to say 
Page 4: STI knowledge - please complete regardless of your reason for attending Student Health

a. How much do you know about sexually transmitted infections (STIs), such as chlamydia, gonorrhoea, herpes, genital warts or syphilis? For example, how they're spread or how to know if you have been infected?
O A lot
O Some
O Only a little
O Nothing at all
O Don't know

b. For each of the following, please indicate if you can get an STI this way or not. If you don't know, please tick "Don't know".

Kissing
O Yes
O No
O Don't know
Oral sex
O Yes
O No
O Don't know
Sexual intercourse (penetrative sex)
O Yes
O No
O Don't know

c. Please indicate whether you think the following methods are effective in preventing the spread of STIs.

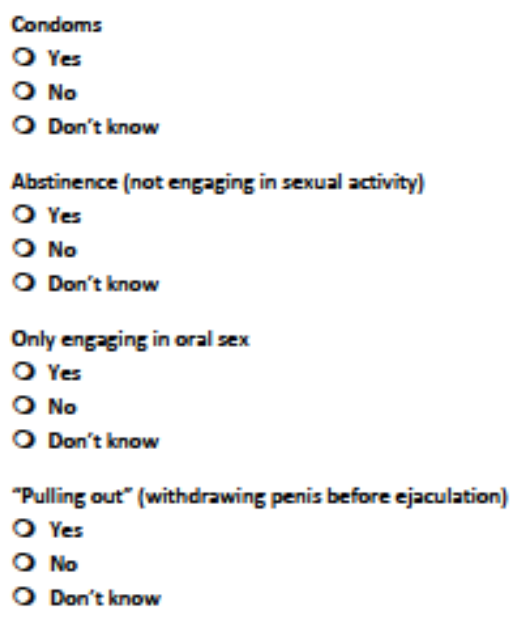

d. Please indicate whether you think the following statements are true or false. if you don't know, please tick "Don't know".
Chlamydia affects only women
O True
O False
Don't know
Chlamydia can lead to infertility in women
O True
O False
O Don't know

Once a person has caught genital herpes, they will always have the virus

O True

O False

O Don't know

Cold sores and genital herpes can be caused by the same virus

O True

O False

Don't know

STls can only be spread when symptoms are present

O True

O False

O Don't know

Gonorrhoea can usually be treated with antibiotics

O True

O False

Don't know

Many STls will cause only mild or no symptoms

O True

O False

O Don't know

Some kinds of cancer can be caused by STls

O True

O False

Don't know

e. Are you aware of any STI campaigns in New Zealand, either now or in the past? These may be campaigns that encourage condom use or STI testing, or that provide information about STIS.

$\square$ Yes - Please give information about what the campaign was and where you heard about it (e.g. TV, radio, posters).

\section{Not aware of any campaigns}

Prize draw entry - Everyone that completes this questionnaire is eligible to be entered into a prize draw.

Please tick this box if you would like to be entered and leave your email address below so that we may contact you if you win.

Email address:

Please tick this box if you are happy to be contacted in relation to the study. This may include a one-question follow-up survey. 
The following information is provided to give an explanation of why and how some of the questionnaire items were chosen and analysed. It is not a comprehensive list, as some of the more straightforward items do not require detailed explanation.

\section{Gender}

The majority of questionnaire tools will collect respondents' gender using a binary female/male question. However, this ignores those that do not identify with this categorisation. Having only female and male options can pose a challenge for transgender people, particularly those that are in the process of, or are considering, transitioning genders (Human Rights Campaign, 2016). Although a large number of individuals identifying as nonbinary was not expected, it was important that the questionnaire was designed in an inclusive manner so as not to offend or exclude any potential participants. On consultation with transgender care providers, it became obvious that there would be too many gender identities to list them all as options on the questionnaire, so a three option design was used: Male, Female, Other (please specify). The 'Other' option had a free text box associated so that individuals could insert their own gender identity without any restrictions imposed by the researcher.

\section{Ethnic group}

Ethnicity is especially important in the New Zealand context due to the unique ethnic makeup of the population, and also due to the history and continuing impact of colonisation. Defining ethnicity is a complex and contentious issue. It is complicated with overlapping definitions of 'race' and 'nationality'. All of these categorisations are socially-derived, but the term 'ethnicity' was chosen to be in line with other New Zealand publications and reports, such as those produced by Statistics New Zealand.

There are a multitude of ways of grouping and defining ethnicity within New Zealand, and many terms are viewed as imperfect. Many options were considered before a final decision was made to follow the Statistics New Zealand census classifications. Participants were able

to select more than one ethnicity as individuals may identify with more than one group. From this question, participants were grouped into five ethnicity categories for data analysis. 


\section{Alcohol use}

Alcohol consumption was measured by the AUDIT-C, which is the first three items from the WHO Alcohol Use Disorders Identification Test (AUDIT) (Saunders et al., 1993, Babor et al., 2001). This tool has been shown to be an effective brief screening test for heavy drinking and/or active alcohol abuse or dependence (Bush et al., 1998), has high validity and internal consistency (Meneses-Gaya et al., 2010), and is widely used in epidemiological research across many settings.

The three questions in the AUDIT-C are:

1. How often did you have a drink containing alcohol?

2. How many standard drinks do you have in a typical day when you are drinking?

3. How often do you have 6 or more standard drinks on one occasion?

Traditionally, the final question has used differing values for men and women. However, as the same questionnaire was to be self-completed by all genders, it was felt to be simpler to use one value in the instructions. This aligns with the recent changes to the UK alcohol guidelines where the recommended limit for weekly alcohol units is now the same for males and females (Department of Health, 2016). Not making any gender distinction also allowed for non-binary individuals to complete the alcohol use questions.

Each question has a corresponding score from 0 to 4 , which are summed to give a total score (scores of 0 reflect no alcohol use). The higher the total score, the more likely it is that the respondent's drinking is heavy and potentially harmful (Bush et al., 1998). Unhealthy alcohol use was classified as an AUDIT-C total score of $\geq 5$ for men and women (Anderson et al., 2016).

\section{Reason for testing}

This multichoice question had 10 options as well as a 'Would prefer not to say' option and an 'Other' option which asked for further details. The options were selected for inclusion from relevant literature (Mercer et al., 2007, Aicken et al., 2011a), and after consulting with clinicians who worked with the patient population about the reasons people typically present for testing. 


\section{Sexually active}

To assess whether a participant had ever been sexually active, the questionnaire simply asked for a tick box answer to the question 'Have you ever been sexually active?' This was based on the report 'Best Practices for Asking Questions about Sexual Orientation on Surveys', which suggests not to use the term 'sexual intercourse' as it is too specific, but rather to allow respondents to decide their own definition (Badgett, 2009).

\section{Sexuality}

Sexuality was not directly asked about in the questionnaire. This was because the outcomes of this study are focussed on behaviour. It is known that same-sex behaviour is underreported when the measure used is sexuality (e.g. are you heterosexual / homosexual / bisexual) (Smith et al., 2003b). Therefore, the questionnaire asked for the number of male and the number of female partners in the past 12 months (and past 3 months for the SHC questionnaire) to obtain a more accurate reflection of same-sex behaviour than would be captured by a sexuality question. 


\section{Appendix B. Developing a recruitment methodology: Results of a focus group study}

\section{Rationale}

An initial pilot study indicated that recruitment through GPs at the SHS for an STI survey would be poor. This prompted some research to better understand students' views about STI testing and research participation in this context. This information was required to aid the design of a recruitment protocol that was acceptable and attractive to this population.

\section{Research question}

What is the most effective method of recruiting VUW students to take part in a survey about STIs?

\section{Research methodology}

Since the purpose of this research was to seek insight into the attitudes, views and opinions of students, the data required were essentially qualitative. A focus group methodology was considered to be the most suitable for this research for several reasons:

1) The research question was focussed on generating ideas, as well as understanding people's feelings and experiences. A focus group is a more creative environment than a one-on-one interview as people can bounce ideas off each other and be inspired by what others are saying.

2) A variety of views, values and opinions needed to be collected in a short time-period. Focus group discussions are a less time-consuming method to gather a range of ideas and opinions than other approaches such as one-on-one interviews.

3) It has been suggested that focus groups are a good methodology for researching taboo subjects (such as STIs) because the less inhibited members of the group can break the ice for shyer participants (Kitzinger, 1995). That said, the research question in itself was not particularly sensitive (focussed on recruitment), so it was felt suitable for a focus group discussion as participants could contribute their ideas without sharing their own experiences if they wished. 
There are a few limitations of focus group discussions that needed to be taken into account. Firstly, it is possible for one or two participants to dominate the discussion which may prevent a group opinion to come through. On the other hand, if there is a majority group consensus about an item of discussion, it may discourage individual voices of dissent to speak up. To avoid these circumstances, the moderators made continued efforts to sustain an open and inclusive atmosphere during all discussions.

\section{Ethical considerations}

Ethical approval for this study was obtained from the Victoria University of Wellington Human Ethics Committee (ref: 20504) (Appendix D). Each participant was emailed a copy of the information sheet and consent form prior to the focus group so they had time to read the study information and ask any questions. On the day of the focus groups, each participant was asked to sign two hard copies of the consent; one for the researcher and one for themselves to take away, and were given another opportunity to ask any questions.

At the beginning of each focus group, the moderators explained that the discussions would be recorded but that no-one would be identified in any dissemination of results. Participants were reminded about the need for confidentiality and asked to respect everyone's privacy. It was also explained that they could leave the discussion at any time if they wanted to, without needing to give a reason.

\section{Guide questions}

A discussion guide with open-ended questions was developed. The guide was flexible and allowed for new questions to be added during the focus group discussions. The focus group discussions started with a short reminder about the purpose of the focus group, followed by asking the participants to imagine that they were in a GP clinic having just had an STI test and to think about their emotions and feelings in that situation. The guiding questions related to the student's perceptions of what is an acceptable study recruitment method in that context (e.g. how do you feel if the doctor or nurse asks you to take part in a survey? Would you prefer to complete a questionnaire on paper or online? Would you prefer to take part at the clinic or later in your own time?). 


\section{Participants}

The target group were students from a university in Wellington. Participants were recruited via posters that were displayed on notice-boards around the University for a period of five days. The posters gave some brief information about the study and had tear off slips containing the lead investigator's email address to contact if they were interested in taking part. No purposeful sampling methodology was undertaken, though participants had to be students to take part.

The number of focus group discussions was chosen a priori as three, due to time constraints. Data saturation analysis was not undertaken during fieldwork as the focus groups all took place on one day. Two mixed-sex and one female-only focus groups were conducted. Two focus groups had five participants each and one had six. This was in line with the recommended group size of between four and eight people (Kitzinger, 1995).

\section{Data collection}

The three focus groups ran over one day in August 2014 in a private room on the university campus. An email was sent a few days prior to the focus group to remind the participants of the time and location, and a final text reminder was sent on the day. Participants were seated on chairs in a circular arrangement around a table so everyone could interact with each other. Refreshments in the form of chocolate were provided.

The discussions were led by two moderators (combination of senior researcher and $\mathrm{PhD}$ student) and lasted between 30 and 45 minutes each. Participants were asked to wear name labels to encourage interaction; participants could chose to write their real name or pseudonym. The focus group discussions were audio-recorded on two separate pieces of recording equipment.

Participants were encouraged to talk to one another and to have a group discussion. At the end of the session, when all questions had been discussed, the moderators summed up the main points of the session and asked participants to say whether they agreed with the summary or if they had anything to change or add. All participants were given a cinema voucher to thank them for their time and contributions. 


\section{Transcription}

The audiotaped focus group discussions were transcribed verbatim by the lead investigator.

\section{Analysis}

Data was analysed using a content analysis methodology. Each transcript was read several times and each line was coded by the lead investigator. From these codes, a coding scheme was developed. The analysis was guided by a constant comparative method, so that codes were tested and used iteratively against the data to produce a set of codes which was grouped into a final set of overall themes.

\section{Results}

From the data, 26 themes were identified which related to the following research questions:

1.) What factors may influence taking part in a study about STIs? (Table AB.1)

2.) What are personal motivators for taking part in research studies? (Table AB.2)

3.) What confidentiality issues should be considered? (Table AB.3)

4.) How should potential participants be approached? (Table AB.4)

5.) How should data be collected? (Table AB.5)

6.) What attributes does a good survey have? (Table AB.6)

7.) Are there any other issues/factors that are relevant? (Table AB.7)

Detailed information about each theme is provided in Tables AB.1 to AB.7; a summary is provided below.

The timing of inviting someone to take part in a research study is important. Various opinions were expressed about the best time to be approached, especially with regards to whether having received the STI test results would positively or negatively influence participation. However, most people felt that a questionnaire was more likely to be completed while waiting for an appointment than if provided at a later time-point.

Some people said they would take part in the study for reasons of altruism, i.e. they wanted to benefit others by taking part in the research. However, other people said that they would be more motivated by an incentive, such as a voucher, and there were various ideas about the necessary size or monetary value of the incentive. 
Confidentiality was mentioned by many people, and it was felt to be a very important aspect of the study. Participants suggested stressing the confidentiality of the study to improve participation. Concerns were raised about only providing questionnaires to those attending for an STI as it may reveal to others in the waiting room that the person was attending for an STI test. Some participants suggested that asking all patients to complete the questionnaire would avoid embarrassment and encourage participation.

There was also considerable discussion around consent to be contacted. The participants felt that if an invitation was to come by email after a consultation, that it should be mentioned first by the doctor at the consultation and that they should have the option to agree or disagree to be contacted.

Paper or online questionnaire picked up in person or emailed were much preferred to an invitation via phone call or SMS text message. Posters were also suggested, although most felt these should be in private areas such as in bathroom stalls.

Participants in the focus groups felt that the questionnaire itself should be quick to answer, specific, educational and use tick boxes or scales, but not long-answer text boxes.

There were some related themes that arose during the discussions, including about the emotions that STI tests elicit (fear, anxiety, stress and embarrassment), that STIs are a sensitive subject, and that there is a lack of STI campaigns in the public domain. Clinicians were also discussed in relation to STI testing, including participants' good and bad experiences, and the differences between doctors and nurses. Social factors relating to STIs, such as stigma and shame, were also frequently mentioned by participants.

\section{Discussion}

Overall, the focus groups indicated that providing a self-complete paper or online questionnaire to complete in the waiting room prior to a consultation at SHS would be a highly acceptable method. In addition, the questionnaire should be general enough that it may be answered by anyone, not only those attending for an STI test.

The results of this work were used to inform the recruitment protocol for the quantitative study at SHS, as described in Chapter 6. 
Table AB.1. Factors which may influence taking part in a questionnaire about STIs

\begin{tabular}{|c|c|c|c|}
\hline Theme & Sub-theme & Descriptors & Example quotes \\
\hline \multirow[t]{5}{*}{ Timing } & $\begin{array}{l}\text { Timing is } \\
\text { important }\end{array}$ & $\begin{array}{l}\text { About the timing of when asked to take part being } \\
\text { directly relevant to whether a person will take part or } \\
\text { not. }\end{array}$ & $\begin{array}{l}\text { I think it depends when you get asked to do them as } \\
\text { well. FG3 L396 }\end{array}$ \\
\hline & Uncertainty & $\begin{array}{l}\text { Includes the idea that not knowing about the nature of a } \\
\text { disease could cause worry. }\end{array}$ & $\begin{array}{l}\text { I think just the fact that you, you're like waiting on } \\
\text { something that's really uncertain could be a factor in } \\
\text { taking it or not kinda thing. FG1 L93 }\end{array}$ \\
\hline & Pressured & $\begin{array}{l}\text { To do with feeling pressured by someone to take part, } \\
\text { relates to timing of being asked. The idea behind these } \\
\text { comments is that by being emailed after, you avoid this } \\
\text { situation. }\end{array}$ & $\begin{array}{l}\text { Not the doctor being like "oh, here's a survey", because } \\
\text { then I would feel kind of pressured to, but if I got an } \\
\text { email it would be in my own time. By then I could kind } \\
\text { of respond to it rather than having someone put you on } \\
\text { the spot, when maybe you've just felt really } \\
\text { uncomfortable. FG1 L99 } \\
\text { You don't have to say yes or no to their face as well, } \\
\text { you can just fill it in. FG2 L } 207\end{array}$ \\
\hline & $\begin{array}{l}\text { Know results } \\
\text { (Timing) }\end{array}$ & $\begin{array}{l}\text { To do with timing. Timing of being asked to take part in } \\
\text { relation to timing of getting the result. How having the } \\
\text { result, and what the result is, would affect taking part. } \\
\text { Also includes how relieved someone feels when result } \\
\text { is negative and how devastating if positive. Some } \\
\text { people would prefer to participate in a study while } \\
\text { waiting for result, others would prefer after. }\end{array}$ & $\begin{array}{l}\text { If it was negative, I would definitely take part. FG1 } \\
\text { L117 } \\
\text { Maybe make sure they'd got their result first, because if } \\
\text { I like had an STI test and then I was waiting for the } \\
\text { results and I got this email, I would be kind of freaking } \\
\text { out. FG3 L131 }\end{array}$ \\
\hline & $\begin{array}{l}\text { Own time/later/ } \\
\text { after }\end{array}$ & To do with timing. Prefer to do it later. & $\begin{array}{l}\text { I just wouldn't do it. If I was stressed and nervous, I'd } \\
\text { be very emotional, I just wouldn't really want to do it at } \\
\text { that point. I'd wanna calm down first. I'd be scared. } \\
\text { FG3 L96 }\end{array}$ \\
\hline
\end{tabular}




\begin{tabular}{|c|c|c|c|}
\hline & $\begin{array}{l}\text { Straight } \\
\text { away/before }\end{array}$ & $\begin{array}{l}\text { About being asked at the start, before the consultation } \\
\text { takes place. } \\
\text { Also includes the feeling that would want to leave } \\
\text { straight away after the consultation. }\end{array}$ & $\begin{array}{l}\text { Like if it's before then you kind of think that it's just } \\
\text { commonplace, like that's what I mean, like afterwards } \\
\text { you're like 'yeah ok this is not really like customary, I } \\
\text { don't really need to do this, I'm not going to worry } \\
\text { about it'. FG2 L60 } \\
\text { I think before I'd probably be more inclined to answer } \\
\text { it, like, rather than afterwards cos I'd think 'oh it's } \\
\text { sorted I don't really need to worry about it' or 'oh my } \\
\text { gosh' FG2 L232 } \\
\text { You kind of just want to get the hell out of there. FG2 } \\
\text { L236 }\end{array}$ \\
\hline Relevance & $\begin{array}{l}\text { Relevant/ applies } \\
\text { to me }\end{array}$ & $\begin{array}{l}\text { About how the feeling of whether the survey applies to } \\
\text { you/is relevant to you, affects participation. The more a } \\
\text { person feels like the study applies to them, the more } \\
\text { likely they are to take part. }\end{array}$ & .... as long as I have a related interest FG3 L380 \\
\hline $\begin{array}{l}\text { Feeling } \\
\text { comfortable }\end{array}$ & Comfort level & $\begin{array}{l}\text { Mentioned quite a lot with the assumption that needs to } \\
\text { be comfortable with the situation to take part in the } \\
\text { research. }\end{array}$ & $\begin{array}{l}\text { I think I'd feel a bit more comfortable if somebody } \\
\text { asked me to take part in research was a nurse rather than } \\
\text { a doctor. FG1 L24 }\end{array}$ \\
\hline $\begin{array}{l}\text { Minimal effort } \\
\text { required }\end{array}$ & Effort & $\begin{array}{l}\text { About how having to do too much/put in a lot of effort } \\
\text { would stop people from doing the questionnaire. }\end{array}$ & $\begin{array}{l}\text { But definitely more I'd do paper version over going to } \\
\text { the link, just cos of the extra effort I guess. FG3 L331 }\end{array}$ \\
\hline Having spare time & Time-pressure & $\begin{array}{l}\text { To do with potential participants being pressed for time, } \\
\text { which may put them off taking part in the study. About } \\
\text { people being more likely to take part if they have spare } \\
\text { time. } \\
\text { Also about making sure participants are aware of the } \\
\text { time involvement. }\end{array}$ & $\begin{array}{l}\text { Even if you had a really strong interest, it's quite a long } \\
\text { time to take out of your day so it would be exceptional } \\
\text { that someone would do it just out of pure altruism. FG1 } \\
\text { L328 } \\
\text { I don't know about you guys, but I find I don't have a } \\
\text { lot of time to do stuff. FG2 L442 }\end{array}$ \\
\hline
\end{tabular}




\section{Table AB.2. Personal motivators for take part in research studies}

\begin{tabular}{|c|c|c|c|}
\hline Theme & Sub-theme & Descriptors & Example quotes \\
\hline \multirow[t]{2}{*}{ Altruism } & Altruism & $\begin{array}{l}\text { The idea that participating in a study is driven by } \\
\text { wanting to help others. }\end{array}$ & $\begin{array}{l}\text { I think just for the sake of other people's benefit, if the } \\
\text { taking part in this will benefit others and it will prevent } \\
\text { others being in your situation then I'd probably be } \\
\text { more inclined to take part. FG1 L123 }\end{array}$ \\
\hline & $\begin{array}{l}\text { Wider } \\
\text { implications of } \\
\text { taking part }\end{array}$ & $\begin{array}{l}\text { Including benefiting other people, for the greater good, } \\
\text { that it's important research. Also in relation to making } \\
\text { sure potential participants are told about this so they can } \\
\text { factor it into their decision to take part. }\end{array}$ & $\begin{array}{l}\text { It's not like you're just taking part in the study, you're } \\
\text { helping with something a bit bigger than that. FG1 } \\
\text { L129 } \\
\text { Knowing how your misfortune can help others would } \\
\text { also be a really good thing to emphasise. FG2 L440 }\end{array}$ \\
\hline Incentive & N/A & $\begin{array}{l}\text { About using incentives to encourage participation (males } \\
\text { seemed to be keener on incentives than females). } \\
\text { Ideas for incentives included movie vouchers, grocery } \\
\text { vouchers, coffee vouchers, chocolate, online credit. } \\
\text { Some discussion over size of incentive, i.e. making sure } \\
\text { it was relative to time involvement; online survey } \\
\text { doesn't necessarily need an incentive but an interview } \\
\text { would. }\end{array}$ & $\begin{array}{l}\text { Yeah, I mean I pretty much only enter ones that have } \\
\text { competitions, because like I don't even notice the other } \\
\text { ones normally. Cos I just don't really pay attention. } \\
\text { Yeah, incentive is crucial, it's a cost benefit thing. FG1 } \\
\text { L325 } \\
\text { I think the nature and the level of incentive is important } \\
\text { though. From what I've seen. FG1 L337 } \\
\text { If it was an online survey, I don't need anything to } \\
\text { make me do it. FG1 L341 } \\
\text { Yeah, I do a lot of online studies, if it's easy I'm like } \\
\text { 'yeah, give me a link, I'll do it'. Well an incentive is } \\
\text { awesome, it's a bonus. FG1 L353 }\end{array}$ \\
\hline
\end{tabular}


Table AB.3. Confidentiality considerations

\begin{tabular}{|l|l|l|l|}
\hline \multicolumn{1}{|c|}{ Theme } & \multicolumn{1}{|c|}{ Sub-theme } & \multicolumn{1}{c|}{ Descriptors } & \multicolumn{1}{c|}{ Example quotes } \\
\hline Privacy & Confidentiality & $\begin{array}{l}\text { Participants often mentioned confidentiality } \\
\text { spontaneously and stressed its importance. }\end{array}$ & $\begin{array}{l}\text { As long as it's confidential, and people don't see that } \\
\text { your name's up there. FG2 L93 }\end{array}$ \\
\cline { 2 - 4 } & Discreet/privacy & About being discreet. About nobody seeing. & $\begin{array}{l}\text { Yeah, cos it's completely private and no-one will ever } \\
\text { know. FG1 L291 [in relation to posters on the back of } \\
\text { toilet doors] }\end{array}$ \\
\cline { 2 - 4 } & Anonymous & About the wish for participation to be anonymous & $\begin{array}{l}\text { If it was an anonymous thing, if there wasn't any more } \\
\text { embarrassment caused by the survey, then that would } \\
\text { be fine I think. FG3 L417 }\end{array}$ \\
\hline Permissions & $\begin{array}{l}\text { Permission to } \\
\text { contact }\end{array}$ & $\begin{array}{l}\text { To do with how the students would like to be } \\
\text { approached to take part. They need to have given prior } \\
\text { permission to contact them (e.g. to the Dr or something). } \\
\text { piece of paper. I might not necessarily keep it and look } \\
\text { at it, but if I was told about it then, and then maybe } \\
\text { said yeah sure, maybe I'd be interested, can they } \\
\text { contact you and then get an email or a text, like that } \\
\text { would maybe be ok. FG1 L243 }\end{array}$ \\
\hline
\end{tabular}


Table AB.4. Mode of approach

\begin{tabular}{|l|l|l|l|}
\hline \multicolumn{1}{|c|}{ Theme } & \multicolumn{1}{|c|}{ Sub-theme } & \multicolumn{1}{c|}{ Descriptors } \\
\hline Phone call & N/A & $\begin{array}{l}\text { About having the invitation via phone call. Unanimous } \\
\text { feeling was that it was bad. }\end{array}$ & $\begin{array}{l}\text { Nah. I never answer my phone. For a university } \\
\text { student, like half the time I'm in the library, it's } \\
\text { annoying to get a phone call. Like either a text or an } \\
\text { email. I hate people calling me. FG1 L205 }\end{array}$ \\
\hline Texting & N/A & $\begin{array}{l}\text { I think I'd be worried that a phone call meant I'd got a } \\
\text { positive test result, like they couldn't text it to you so } \\
\text { they've got to ring you. (General agreement from the } \\
\text { group) FG1L207 }\end{array}$ \\
\hline Email & N/A & $\begin{array}{l}\text { About having the invitation via text. Preferred to phone } \\
\text { call but still not much liked. }\end{array}$ & I'd ignore it, the text. FG3 L287 \\
\hline Poster & N/A & $\begin{array}{l}\text { About having the invitation via email. Generally } \\
\text { preferred to all other methods. }\end{array}$ & $\begin{array}{l}\text { About the study/other studies being advertised on } \\
\text { posters. Some reference to working in girls toilets but } \\
\text { not boys as they would just vandalise it (FG2 L 450) }\end{array}$ \\
\hline
\end{tabular}


Table AB.5. Method of data collection

\begin{tabular}{|c|c|c|c|}
\hline Theme & Sub-theme & Descriptors & Example quotes \\
\hline $\begin{array}{l}\text { Online } \\
\text { questionnaire }\end{array}$ & N/A & About preferring online & $\begin{array}{l}\text { Online would probably be good as well, because } \\
\text { people feel like it's private on their phone or tablet or } \\
\text { whatever, rather than on paper which people might be } \\
\text { able to see. FG2 L72 } \\
\text { I'd be more worried about paper, because people can } \\
\text { see paper. FG2 } 110 \\
\text { I'd be more comfortable doing it on my phone. FG2 } \\
\text { L113 }\end{array}$ \\
\hline Paper questionnaire & N/A & $\begin{array}{l}\text { About preferring paper. About doing the questionnaire } \\
\text { in the waiting room. }\end{array}$ & $\begin{array}{l}\text { I'd probably prefer it on paper FG2 L87 } \\
\text { As long as your name wasn't really on it. FG2 L96 } \\
\text { But like, when you go in for a check-up it's not like } \\
\text { you're gonna be sat next to someone, usually it's like } \\
\text { you're all spaced out. FG2 L126 }\end{array}$ \\
\hline Give options & N/A & $\begin{array}{l}\text { About giving people the option of paper or online. And } \\
\text { giving them the option of filling it out before and after. } \\
\text { Both ideas arose spontaneously. }\end{array}$ & $\begin{array}{l}\text { You can always give people the option too, when they } \\
\text { get there. Like, 'there's this link or you can do it on } \\
\text { paper'. FG2 L114 } \\
\text { Would it be possible to have a choice of the two survey } \\
\text { delivery methods, cos that might be the kind of } \\
\text { deciding factor for some people, like whether they } \\
\text { could do it online or whether they could do it right } \\
\text { then? FG2 L265 }\end{array}$ \\
\hline
\end{tabular}


Table AB.6. Attributes of a good survey

\begin{tabular}{|l|l|l|l|}
\multicolumn{1}{|c|}{ Theme } & \multicolumn{1}{|c|}{ Sub-theme } & \multicolumn{1}{c|}{ Descriptors } \\
\hline Specific & N/A & $\begin{array}{l}\text { About wanting the questions to be specific and easy to } \\
\text { answer. }\end{array}$ & $\begin{array}{l}\text { I like yes/no, like straight to the point, like quickly, like } \\
\text { you don't have to think too much about it. FG2 L328 }\end{array}$ \\
\hline Educational & N/A & $\begin{array}{l}\text { About how learning from questionnaires is useful and } \\
\text { appreciated. }\end{array}$ & $\begin{array}{l}\text { You could learn some stuff about STIs, that would be } \\
\text { good. FG3 L402 }\end{array}$ \\
\hline Quick & N/A & $\begin{array}{l}\text { Refers to participants liking quick surveys including } \\
\text { quick description of the survey and quick instructions. }\end{array}$ & $\begin{array}{l}\text { If I can read quickly what it's going to be used for and } \\
\text { I agree with it then I'll take part in it, otherwise I just } \\
\text { ignore it. FG2 L365 }\end{array}$ \\
\hline Tick boxes & N/A & $\begin{array}{l}\text { Participants expressed that they prefer tick boxes on } \\
\text { questionnaires to other forms of answering questions. }\end{array}$ & $\begin{array}{l}\text { Like I personally hate the ones where they're like... } \\
\text { they'll get you to tick something and then they're like } \\
\text { 'please comment on your answer' and then they'll get } \\
\text { you to write why you chose what you chose and what } \\
\text { your answer is... I just skip it, if there's a tick option } \\
\text { I'll tick it and if they're like 'please provide detail', } \\
\text { I'm just like 'nah'. FG3 L383 }\end{array}$ \\
\hline Scales & N/A & $\begin{array}{l}\text { Cos sometimes it's really hard to... some scales don't } \\
\text { really represent what the answer you... yeah. But } \\
\text { giving the option of a scale and a comment if you want. } \\
\text { FG3 L392 }\end{array}$ \\
\hline
\end{tabular}


Table AB.7. Related themes

\begin{tabular}{|c|c|c|c|}
\hline Theme & Sub-theme & Descriptors & Example quotes \\
\hline \multirow{4}{*}{$\begin{array}{l}\text { Feelings about STIs } \\
\text { and having an STI } \\
\text { test }\end{array}$} & Fear & $\begin{array}{l}\text { Feeling panicked about the situation of the STI test and } \\
\text { possible result }\end{array}$ & $\begin{array}{l}\text { You automatically think worst case scenario "I'm } \\
\text { gonna die, that's it". FG2 L48 }\end{array}$ \\
\hline & Anxious & $\begin{array}{l}\text { Worry over having an STI, and about the STI result. } \\
\text { Includes the idea that we wouldn't want the survey to } \\
\text { cause more worry and anxiety. }\end{array}$ & $\begin{array}{l}\text { I just wouldn't do it. If I was stressed and nervous, I'd } \\
\text { be very emotional, I just wouldn't really want to do it } \\
\text { at that point. I'd wanna calm down first. I'd be scared. } \\
\text { FG } 3 \text { L96 }\end{array}$ \\
\hline & Stress & $\begin{array}{l}\text { Includes the idea that Uni students may already be } \\
\text { stressed and this research could add to it. }\end{array}$ & $\begin{array}{l}\text { For people that are already stressing in a position } \\
\text { where they can't deal with bad news, it could just make } \\
\text { things more difficult and it could just scare them off } \\
\text { totally. FG1 L144 }\end{array}$ \\
\hline & Embarrassed & $\begin{array}{l}\text { Refers to being embarrassed about going for a test or } \\
\text { being embarrassed about having an STI. Also about } \\
\text { making sure the survey doesn't cause more } \\
\text { embarrassment for people. }\end{array}$ & $\begin{array}{l}\text { Maybe a little embarrassed, like "Oh my god, I've got a } \\
\text { disease". Well you know, not a socially acceptable one. } \\
\text { FG1 L12 } \\
\text { Participant: I've supported someone going in, she had a } \\
\text { positive one and she had to go into treatment and I } \\
\text { went in with her, and she cried the whole time. } \\
\text { Facilitator: What was she crying about? } \\
\text { Participant: She was embarrassed FG1 L178 }\end{array}$ \\
\hline \multirow[t]{2}{*}{$\begin{array}{l}\text { Perceptions about } \\
\text { STIs }\end{array}$} & $\begin{array}{l}\text { Serious/ } \\
\text { Consequences }\end{array}$ & $\begin{array}{l}\text { The idea that some STIs can be very serious. } \\
\text { Participants considered the consequences of having an } \\
\text { STI. Fertility and impact on sex life mentioned. }\end{array}$ & $\begin{array}{l}\text { And maybe the consequences might be if you've got an } \\
\text { STI, what the consequences might be to your body? } \\
\text { Like fertility, um what it might do you, um, yeah, the } \\
\text { treatment... FG2 L50 }\end{array}$ \\
\hline & Sensitive subject & About STIs being a sensitive issue for people. & $\begin{array}{l}\text { Cos with it being sort of a sensitive survey maybe it's } \\
\text { better coming to someone in person first. FG3 L145 }\end{array}$ \\
\hline
\end{tabular}




\begin{tabular}{|c|c|c|c|}
\hline \multirow{2}{*}{$\begin{array}{l}\text { Societal influence } \\
\text { on having an STI } \\
\text { test/taking part in } \\
\text { the study }\end{array}$} & Shame & Shame about going for an STI test. & $\begin{array}{l}\text { There's an element of shame in asking for a test } \\
\text { because, you know, it's quite a sensitive issue. FG2 } \\
\text { L27 }\end{array}$ \\
\hline & $\begin{array}{l}\text { Stigma and others } \\
\text { knowing }\end{array}$ & $\begin{array}{l}\text { About being judged in relation to having an STI/having } \\
\text { an STI test. About being scared of being judged (mainly } \\
\text { in terms of women being scared of being judged - this } \\
\text { comment came from both males and females) } \\
\text { Includes about women being "slut-shamed" (discussed } \\
\text { in FG3, female only group). } \\
\text { About people seeing someone is there for an STI test, or } \\
\text { seeing them take part in the study. } \\
\text { Includes feeling that wouldn't want to hand the } \\
\text { questionnaire in to receptionist. Although FG3 (all girl } \\
\text { group) were happy to hand into reception. }\end{array}$ & $\begin{array}{l}\text { Maybe a little embarrassed, like "Oh my god, I've got a } \\
\text { disease". Well you know, not a socially acceptable one. } \\
\text { FG1 L12 } \\
\text { You go out for a piss test and people can see you're } \\
\text { going out for a piss test... that was the main awkward } \\
\text { thing. FG1 L43 } \\
\text { I think that would be a bit awkward for some people, } \\
\text { just having to hand it in in person and being seen to } \\
\text { hand it in, yeah, that might be awkward for some } \\
\text { people. Personally, I don't really care but I know that } \\
\text { some people are worried about that stuff. FG1 L256 }\end{array}$ \\
\hline \multirow[t]{2}{*}{$\begin{array}{l}\text { Clinician's part in } \\
\text { STI test/taking part } \\
\text { in the study }\end{array}$} & $\begin{array}{l}\text { Power } \\
\text { relationship } \\
\text { between clinician } \\
\text { and patient }\end{array}$ & $\begin{array}{l}\text { Between doctor and patient. Generally commented upon, } \\
\text { but also discussed in the context of how to approach } \\
\text { someone to take part - people do what the doctor tells } \\
\text { them to. }\end{array}$ & $\begin{array}{l}\text { Maybe there's not such a degree of difference in power } \\
\text { between you and the nurse and you and the doctor. } \\
\text { FG1 L34 } \\
\text { Another option I thought of was maybe, I don't know if } \\
\text { this is even legal, but like you go into the office and the } \\
\text { doctor asks you about your things and they're like 'oh } \\
\text { can you just fill out this survey?' and cos like, I just do } \\
\text { what a doctor tells me to do. FG3 L173 }\end{array}$ \\
\hline & $\begin{array}{l}\text { Clinician } \\
\text { behaviour/ } \\
\text { attitude }\end{array}$ & $\begin{array}{l}\text { The mannerism of the clinician. Includes about past } \\
\text { experience of professionalism. Includes wanting a } \\
\text { balance between sympathy and educating. Includes } \\
\text { wanting clinician to be open and to be relaxed. Some } \\
\text { discussion about gender of clinician in FG1, but } \\
\text { generally not thought of as too important other than by } \\
\text { some of the females who would prefer a female } \\
\text { clinician. }\end{array}$ & $\begin{array}{l}\text { It kinda depends on like how the doctor treats it, cos } \\
\text { like I've been to doctors before, like this is just for } \\
\text { random stuff, and like they can either be like really } \\
\text { nice or really like "ohh, that was stupid of you" and } \\
\text { make you feel embarrassed. FG } 3 \text { L20 }\end{array}$ \\
\hline $\begin{array}{l}\text { Lack of STI } \\
\text { campaigns }\end{array}$ & $\begin{array}{l}\text { Lack of } \\
\text { campaigns }\end{array}$ & $\begin{array}{l}\text { People said they didn't know of many campaigns. They } \\
\text { hadn't had very good sex education, and mainly learnt } \\
\text { about sexual health from their friends }\end{array}$ & $\begin{array}{l}\text { The only thing I've heard about STIs at Uni is one of } \\
\text { the courses we do, Biology 116, so there was like a big } \\
\text { bit on STDs, so yeah. FG2 L474 }\end{array}$ \\
\hline
\end{tabular}




\section{Appendix C. Supplementary tables for Chapter 6}

Table AC.1. Difference in knowledge by sociodemographic and sexual behaviour characteristics for SHS

\begin{tabular}{|c|c|c|c|c|c|}
\hline \multirow[b]{2}{*}{ Characteristic } & \multicolumn{5}{|c|}{ Student Health Services } \\
\hline & $n$ & Mean rank & $U$ & $Z$ & $p$ value \\
\hline Alcohol use & & & 11768.500 & -2.794 & 0.005 \\
\hline AUDIT-C score $<5$ & 159 & 154.02 & & & \\
\hline AUDIT-C score $\geq 5$ & 179 & 183.25 & & & \\
\hline Smoking & & & 5622.000 & -2.716 & 0.007 \\
\hline Yes & 51 & 203.76 & & & \\
\hline No & 288 & 164.02 & & & \\
\hline Relationship status & & & 1680.500 & -2.051 & 0.040 \\
\hline Single & 66 & 72.04 & & & \\
\hline In a current relationship & 64 & 58.76 & & & \\
\hline Condom use & & & 1869.000 & -0.523 & 0.601 \\
\hline Frequent & 50 & 67.12 & & & \\
\hline Infrequent & 79 & 63.66 & & & \\
\hline Sexual orientation behaviour in men & & & 53.500 & -0.936 & 0.349 \\
\hline Non-MSM & 20 & 13.18 & & & \\
\hline MSM & 7 & 16.36 & & & \\
\hline Sexual orientation behaviour in women & & & 165.000 & -0.215 & 0.830 \\
\hline Non-WSW & 88 & 46.38 & & & \\
\hline \multirow[t]{2}{*}{ WSW } & 4 & 49.25 & & & \\
\hline & $n$ & Mean rank & $H(d f)$ & & $p$ value \\
\hline Gender & & & 0.690 & & 0.844 \\
\hline Male & 102 & 167.52 & & & \\
\hline Female & 229 & 165.32 & & & \\
\hline Other & 8 & 197.13 & & & \\
\hline Age & & & $1.692(2)$ & & 0.429 \\
\hline$<25$ & 269 & 163.00 & & & \\
\hline $25-34$ & 43 & 166.33 & & & \\
\hline $35+$ & 17 & 193.32 & & & \\
\hline Ethnicity & & & $1.103(4)$ & & 0.894 \\
\hline Māori & 33 & 168.65 & & & \\
\hline Pacific peoples & 3 & 216.83 & & & \\
\hline Asian & 17 & 155.97 & & & \\
\hline Other & 27 & 172.19 & & & \\
\hline \multirow[t]{2}{*}{ New Zealand European } & 256 & 168.36 & & & \\
\hline & & $\begin{array}{l}\text { Spearman's } \\
\text { correlation }\end{array}$ & & & $p$ value \\
\hline Number of sexual partners & 125 & 0.215 & & & 0.016 \\
\hline
\end{tabular}


Table AC.2. Difference in knowledge by sociodemographic and sexual behaviour characteristics for SHC

Sexual Health Clinic

\begin{tabular}{|c|c|c|c|c|c|}
\hline Characteristic & $n$ & Mean rank & $U$ & $Z$ & $p$ value \\
\hline Alcohol use & & & 5793.500 & -1.442 & 0.149 \\
\hline AUDIT-C score $<5$ & 91 & 109.66 & & & \\
\hline AUDIT-C score $\geq 5$ & 143 & 122.49 & & & \\
\hline Smoking & & & 4507.000 & -1.363 & 0.173 \\
\hline Yes & 58 & 127.79 & & & \\
\hline No & 176 & 114.11 & & & \\
\hline Relationship status & & & 5779.000 & -1.894 & 0.058 \\
\hline Single & 132 & 124.72 & & & \\
\hline In a current relationship & 102 & 108.16 & & & \\
\hline Condom use & & & 6210.500 & -1.204 & 0.229 \\
\hline Frequent & 110 & 123.04 & & & \\
\hline Infrequent & 124 & 112.58 & & & \\
\hline Sexual orientation behaviour in men & & & 1111.000 & -1.385 & 0.166 \\
\hline Non-MSM & 83 & 55.39 & & & \\
\hline MSM & 32 & 64.78 & & & \\
\hline Sexual orientation behaviour in women & & & 332.000 & -1.288 & 0.198 \\
\hline Non-WSW & 99 & 53.35 & & & \\
\hline \multirow[t]{2}{*}{ WSW } & 9 & 67.11 & & & \\
\hline & $n$ & Mean rank & $H(d f)$ & & $p$ value \\
\hline Gender & & & 0.095 & & 0.758 \\
\hline Male & 122 & 116.22 & & & \\
\hline Female & 112 & 118.89 & & & \\
\hline Other & 0 & - & & & \\
\hline Age & & & $1.988(2)$ & & 0.370 \\
\hline$<25$ & 100 & 110.46 & & & \\
\hline $25-34$ & 92 & 122.92 & & & \\
\hline $35+$ & 38 & 110.80 & & & \\
\hline Ethnicity & & & $10.611(4)$ & & 0.031 \\
\hline Māori & 16 & 127.72 & & & \\
\hline Pacific peoples & 2 & 59.75 & & & \\
\hline Asian & 12 & 64.42 & & & \\
\hline Other & 94 & 123.19 & & & \\
\hline \multirow[t]{2}{*}{ New Zealand European } & 107 & 114.77 & & & \\
\hline & & $\begin{array}{l}\text { Spearman's } \\
\text { correlation }\end{array}$ & & & $p$ value \\
\hline Number of sexual partners & 219 & 0.133 & & & 0.049 \\
\hline
\end{tabular}


Table AC.3. The effect of sociodemographic variables on total knowledge score for the SHS sample $(n=319)$

\begin{tabular}{lccc}
\hline Characteristic & OR & $95 \%$ CI & $p$ value \\
\hline Gender & & & 0.663 \\
$\quad$ Male & 0.963 & $0.621-1.492$ & 0.865 \\
$\quad$ Other & 1.834 & $0.469-7.175$ & 0.383 \\
$\quad$ Female & 1 & & \\
Age & & & 0.172 \\
$\quad<25$ & 0.420 & $0.167-1.058$ & 0.066 \\
$25-34$ & 0.503 & $0.177-1.433$ & 0.198 \\
$\quad 35+$ & 1 & & \\
Ethnicity & & & 0.938 \\
$\quad$ Māori & 0.912 & $0.468-1.778$ & 0.788 \\
$\quad$ Pacific peoples & 1.533 & $0.197-11.906$ & 0.683 \\
$\quad$ Asian & 0.785 & $0.326-1.893$ & 0.590 \\
$\quad$ Other & 1.180 & $0.569-2.444$ & 0.657 \\
$\quad$ New Zealand European & 1 & & \\
Alcohol use & & & \\
$\quad$ AUDIT-C score $<5$ & 0.631 & $0.415-0.960$ & 0.032 \\
$\quad$ AUDIT-C score $\geq 5$ & 1 & & \\
Smoking & & & \\
$\quad$ No & 0.543 & $0.311-0.947$ & 0.031 \\
$\quad$ Yes & 1 & & \\
\hline
\end{tabular}

Table AC.4. The effect of sociodemographic variables on total knowledge score for the SHC sample $(n=227)$

\begin{tabular}{lccc}
\hline Characteristic & OR & $95 \%$ CI & $p$ value \\
\hline Gender & & & \\
$\quad$ Male & 0.896 & $0.548-1.465$ & 0.661 \\
$\quad$ Female & 1 & & \\
$\quad$ Other & - & - & - \\
Age & & & 0.504 \\
$\quad<25$ & 0.851 & $0.407-1.777$ & 0.667 \\
$\quad 25-34$ & 1.170 & $0.566-2.418$ & 0.672 \\
$\quad 35+$ & 1 & & \\
Ethicity & & & 0.009 \\
$\quad$ Māori & 1.420 & $0.525-3.836$ & 0.490 \\
$\quad$ Pacific peoples & 0.271 & $0.022-3.294$ & 0.306 \\
$\quad$ Asian & 0.162 & $0.053-0.497$ & 0.001 \\
$\quad$ Other & 1.204 & $0.710-2.043$ & 0.491 \\
$\quad$ New Zealand European & 1 & & \\
Alcohol use & & & \\
$\quad$ AUDIT-C score $<5$ & 0.842 & $0.490-1.445$ & 0.532 \\
$\quad$ AUDIT-C score $\geq 5$ & 1 & & \\
Smoking & & & \\
$\quad$ No & 0.810 & $0.458-1.430$ & 0.467 \\
$\quad$ Yes & 1 & & \\
\hline
\end{tabular}


Table AC.5. Ordinal regression of total knowledge score with sexual behaviour characteristics added into the model, for both samples combined $(n=331)$

\begin{tabular}{|c|c|c|c|}
\hline Characteristic & OR & $95 \% \mathrm{CI}$ & $p$ value \\
\hline \multicolumn{4}{|l|}{ Gender } \\
\hline Male & 1.235 & $0.152-10.029$ & 0.843 \\
\hline Female & 1.305 & $0.163-10.437$ & 0.802 \\
\hline Other & 1 & & \\
\hline \multicolumn{4}{|l|}{ Age } \\
\hline$<25$ & 0.755 & $0.377-1.511$ & 0.427 \\
\hline $25-34$ & 0.822 & $0.400-1.686$ & 0.592 \\
\hline $35+$ & 1 & & \\
\hline \multicolumn{4}{|l|}{ Ethnicity } \\
\hline Māori & 1.323 & $0.614-2.848$ & 0.475 \\
\hline Pacific peoples & 0.742 & $0.098-5.602$ & 0.772 \\
\hline Asian & 0.225 & $0.088-0.574$ & 0.002 \\
\hline Other & 1.324 & $0.825-2.123$ & 0.244 \\
\hline New Zealand European & 1 & & \\
\hline \multicolumn{4}{|l|}{ Alcohol use } \\
\hline AUDIT-C score $<5$ & 0.788 & $0.500-1.242$ & 0.305 \\
\hline AUDIT-C score $\geq 5$ & 1 & & \\
\hline \multicolumn{4}{|l|}{ Smoking } \\
\hline No & 0.678 & $0.411-1.120$ & 0.129 \\
\hline Yes & 1 & & \\
\hline \multicolumn{4}{|l|}{ Relationship status } \\
\hline Single & 1.442 & $0.958-2.172$ & 0.079 \\
\hline In a relationship & 1 & & \\
\hline \multicolumn{4}{|l|}{ Condom use } \\
\hline Frequent & 1.211 & $0.811-1.808$ & 0.349 \\
\hline Infrequent & 1 & & \\
\hline Number of sexual partners in past 12 months & 1.016 & $0.977-1.056$ & 0.430 \\
\hline
\end{tabular}




\section{Appendix D. Ethical approval confirmations}

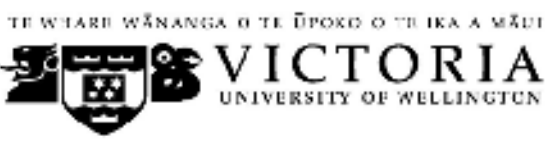

$\begin{array}{ll} & \text { Phone } \\ \text { MEMORANDUM } & \text { Fax } \\ \text { Email }\end{array}$

\begin{tabular}{l|l}
\hline TO & Hayley Denison \\
\hline COPY TO & Elaine Dennison \\
\hline FROM & Dr Allison Kirkman, Convener, Human Ethics Committee \\
\hline
\end{tabular}

\begin{tabular}{l|l}
\hline DATE & 13 December 2013 \\
\hline PAGES & 1 \\
\hline
\end{tabular}

SUBJECT $\quad$ Ethics Approval: 20504

Do students delay their healthcare-seeking for Sexually

Transmitted Infection testing and does this have implications for infection transmission and health-outcomes?

Thank you for your application for ethical approval, which has now been considered by the Standing Committee of the Human Ethics Committee.

Your application has been approved from the above date and this approval continues until 31 August 2016. If your data collection is not completed by this date you should apply to the Human Ethics Committee for an extension to this approval.

Best wishes with the research.

Allison Kirkman

Human Ethics Committee 
IL WHAHE NÄNANGA O TE CHOKO O TE IKA A MABLL

9葸8 YICTORIA

\section{MEMORANDUM}

\begin{tabular}{l|l}
\hline TO & Hayley Denison \\
\hline COPY TO & $\begin{array}{l}\text { Annemarie Jutel } \\
\text { Elaine Dennison }\end{array}$ \\
\hline FROM & AProf Susan Corbett, Convener, Human Ethics Committee \\
\hline \multicolumn{2}{|l}{17 August 2015 } \\
\hline DATE & 1 \\
\hline PAGES & $\begin{array}{l}\text { Ethics Approval: 22110 } \\
\text { Pathways to presentation for sexually transmitted infection testing } \\
\text { among a University population: a qualitative study }\end{array}$ \\
\hline
\end{tabular}

Thank you for your application for ethical approval, which has now been considered by the Standing Committee of the Human Ethics Committee.

Your application has been approved from the above date and this approval continues until 30 November 2016. If your data collection is not completed by this date you should apply to the Human Ethics Committee for an extension to this approval.

Best wishes with the research.

Kind regards

\section{Susan Corbett}

Convener, Victoria University Human Ethics Committee 


\section{References}

1. Abel G, Brunton C. Young people's use of condoms and their perceived vulnerability to sexually transmitted infections. Aust N Z J Public Health. 2005;29(3):254-60.

2. Abusarah EA, Awwad ZM, Charvalos E, Shehabi AA. Molecular detection of potential sexually transmitted pathogens in semen and urine specimens of infertile and fertile males. Diagn Microbiol Infect Dis. 2013;77(4):283-6.

3. Adachi K, Nielsen-Saines K, Klausner JD. Chlamydia trachomatis Infection in Pregnancy: The Global Challenge of Preventing Adverse Pregnancy and Infant Outcomes in Sub-Saharan Africa and Asia. Biomed Res Int. 2016;2016:[Epub ahead of print].

4. Adams EJ, Charlett A, Edmunds WJ, Hughes G. Chlamydia trachomatis in the United Kingdom: a systematic review and analysis of prevalence studies. Sex Transm Infect. 2004;80(5):354-62.

5. Aggarwal R, Ringold S, Khanna D, Neogi T, Johnson SR, Miller A, et al. Distinctions between diagnostic and classification criteria? Arthritis Care Res (Hoboken). 2015;67(7):8917.

6. Agius PA, Pitts MK, Smith AM, Mitchell A. Sexual behaviour and related knowledge among a representative sample of secondary school students between 1997 and 2008. Aust N Z J Public Health. 2010;34(5):476-81.

7. Ahvonen P, Sievers K, Aho K. Arthritis associated with Yersinia enterocolitica infection. Acta Rheumatol Scand. 1969;15(3):232-53.

8. Aicken CR, Cassell JA, Estcourt CS, Keane F, Brook G, Rait G, et al. Rationale and development of a survey tool for describing and auditing the composition of, and flows between, specialist and community clinical services for sexually transmitted infections. BMC Health Serv Res. 2011a;11:30.

9. Aicken CR, Nardone A, Mercer CH. Alcohol misuse, sexual risk behaviour and adverse sexual health outcomes: evidence from Britain's national probability sexual behaviour surveys. J Public Health (Oxf). 2011b;33(2):262-71.

10. AIDS Epidemiology Group. AIDS - New Zealand, Issue 75. Available at http://dnmeds.otago.ac.nz/departments/psm/research/aids/pdf/75\%20AIDSNZ\%20May\%202016.pdf [Accessed 01/12/16]. 2016.

11. AIDS Epidemiology Group. Epidemiological surveillance of HIV/AIDS. Epidemiological surveillance of HIV/AIDS. Available at: http://dnmeds.otago.ac.nz/departments/psm/research/aids/epi_surveill.html [Accessed 01/12/16]. 2012.

12. Ajene AN, Fischer Walker CL, Black RE. Enteric pathogens and reactive arthritis: a systematic review of Campylobacter, salmonella and Shigella-associated reactive arthritis. $J$ Health Popul Nutr. 2013;31(3):299-307. 
13. Ajzen I. The theory of planned behavior. Organ Behav Hum Decis Process. 1991;50(2):179211.

14. Akinnawo EO, Oguntimehin F. Health-seeking behaviour of STD patients in an urban area of southwest Nigeria: an exploratory study. Health Transit Rev. 1997;7 Suppl:307-13.

15. Alexander B. At-home STD kits private - but sometimes wrong (webpage). Available at: http://www.nbcnews.com/id/42599128/ns/health-sexual_health/t/at-home-std-tests-privatesometimes-wrong/\#.WENjqdJ96Uk [Accessed 04/12/16]. 2011.

16. Allen L. 'Say everything': exploring young people's suggestions for improving sexuality education. Sex Education. 2005;5(4):389-404.

17. Allen L. Doing 'it' differently: relinquishing the disease and pregnancy prevention focus in sexuality education. Br J Sociol Educ. 2007;28(5):575-88.

18. Amor B, Dougados M, Mijiyawa M. Criteria of the classification of spondylarthropathies. Rev Rhum Mal Osteoartic. 1990;57(2):85-9.

19. Anderson DJ, Marathe J, Pudney J. The structure of the human vaginal stratum corneum and its role in immune defense. Am J Reprod Immunol. 2014;71(6):618-23.

20. Anderson P, Bendtsen P, Spak F, Reynolds J, Drummond C, Segura L, et al. Improving the delivery of brief interventions for heavy drinking in primary health care: outcome results of the Optimizing Delivery of Health Care Intervention (ODHIN) five-country cluster randomized factorial trial. Addiction. 2016;111(11):1935-45.

21. Andersson-Ellstrom A, Milsom I. Knowledge about the prevention of sexually transmitted diseases: a longitudinal study of young women from 16-23 years of age. Sex Transm Infect. 2002;78(5):339-41.

22. Aral SO. Understanding racial-ethnic and societal differentials in STI. Sex Transm Infect. 2002;78(1):2-4.

23. Aral SO, Over, M., Manhart, L., Holmes, K. K. Chapter 17 - Sexually Transmitted Infections. In: Jamison DT, Breman, J. G., Measham, A. R., Alleyne, G., Claeson, M., Evans, D. B., Jha, P., Mills, A., Musgrove, P., editor. Disease Control Priorities in Developing Countries (Second Edition): World Bank, Washington (DC); 2006.

24. Arbeit MR, Fisher CB, Macapagal K, Mustanski B. Bisexual Invisibility and the Sexual Health Needs of Adolescent Girls. LGBT Health. 2016;3(5):342-9.

25. Ashby B, Gupta S. Sexually transmitted infections in polygamous mating systems. Philos Trans R Soc Lond B Biol Sci. 2013;368(1613):20120048.

26. Auerbach J. Transforming social structures and environments to help in HIV prevention. Health Aff (Millwood). 2009;28(6):1655-65.

27. Auerbach JD, Coates TJ. HIV prevention research: accomplishments and challenges for the third decade of AIDS. Am J Public Health. 2000;90(7):1029-32. 
28. Australasian Society for HIV Medicine. HIV, viral hepatitis \& STIs. A guide for primary care providers. 2014.

29. Azariah S, McKernon S, Werder S. Large increase in opportunistic testing for chlamydia during a pilot project in a primary health organisation. J Prim Health Care. 2013;5(2):141-5.

30. Azariah S, Perkins N. Risk factors and characteristics of patients with gonorrhoea presenting to Auckland Sexual Health Service, New Zealand. N Z Med J. 2007;120(1252):U2491.

31. Babor TF, Higgins-Biddle JC, Saunders JB, Monteiro MG. The Alcohol Use Disorders Identification Test Guidelines for Use in Primary Care (Second Edition). Geneva: World Health Organization, 2001.

32. Badgett MVL, and the Sexual Minority Assessment Research Team (SMART). Best Practices for Asking Questions about Sexual Orientation on Surveys. The Williams Institute 2009.

33. Baggaley RF, White RG, Boily MC. HIV transmission risk through anal intercourse: systematic review, meta-analysis and implications for HIV prevention. Int J Epidemiol. 2010;39(4):1048-63.

34. Baker M, Ortega-Benito J, Garret N, Bromhead C, Leslie K, MacDonald J, et al. Prevalence and risk factors for Chlamydia trachomatis infection in female New Zealand university students. N Z Med J. 2005;118(1220):U1607.

35. Baker MG, Barnard LT, Kvalsvig A, Verrall A, Zhang J, Keall M, et al. Increasing incidence of serious infectious diseases and inequalities in New Zealand: a national epidemiological study. Lancet. 2012;379(9821):1112-9.

36. Balfe M, Brugha R. What prompts young adults in Ireland to attend health services for STI testing? BMC Public Health. 2009;9:311.

37. Balfe M, Brugha R. Disclosure of STI testing activities by young adults: the influence of emotions and social networks. Sociol Health Illn. 2010;32(7):1041-58.

38. Balfe M, Brugha R, O' Connell E, McGee H, O' Donovan D. Where do young Irish women want Chlamydia-screening services to be set up? A qualitative study employing Goffman's impression management framework. Health Place. 2010a;16(1):16-24.

39. Balfe M, Brugha R, O' Donovan D, O' Connell E, Vaughan D. Triggers of self-conscious emotions in the sexually transmitted infection testing process. BMC Res Notes. 2010b;3:229.

40. Balfe M, Brugha R, O'Connell E, Vaughan D, O'Donovan D. Men's attitudes towards chlamydia screening: a narrative review. Sexual Health. 2012;9(2):120-30.

41. Balfe M, Brugha R, O'Donovan D, O'Connell E, Vaughan D. Young women's decisions to accept chlamydia screening: influences of stigma and doctor-patient interactions. BMC Public Health. 2010c;10:425.

42. Baliunas D, Rehm J, Irving H, Shuper P. Alcohol consumption and risk of incident human immunodeficiency virus infection: a meta-analysis. Int J Public Health. 2010;55(3):159-66. 
43. Bannon W, Jr. Missing data within a quantitative research study: How to assess it, treat it, and why you should care. J Am Assoc Nurse Pract. 2015;27(4):230-2.

44. Barker DJ. The fetal and infant origins of disease. Eur J Clin Invest. 1995;25(7):457-63.

45. Barker DJ, Winter PD, Osmond C, Margetts B, Simmonds SJ. Weight in infancy and death from ischaemic heart disease. Lancet. 1989;2(8663):577-80.

46. Barsky AJ, 3rd. Hidden reasons some patients visit doctors. Ann Intern Med. 1981;94:492-8.

47. Barth KR, Cook RL, Downs JS, Switzer GE, Fischhoff B. Social stigma and negative consequences: factors that influence college students' decisions to seek testing for sexually transmitted infections. J Am Coll Health. 2002;50(4):153-9.

48. Bartlett JG. Human immunodeficiency virus infection. New Horiz. 1993;1(2):302-11.

49. Barzon L, Trevisan M, Sinigaglia A, Lavezzo E, Palu G. Zika virus: from pathogenesis to disease control. FEMS Microbiol Lett. 2016;363(18).

50. BASHH. United Kingdom National Guideline for Gonorrhoea Testing. Clinical Effectiveness Group. British Association of Sexual Health and HIV, 2012.

51. Basu I, Bromhead C, Balm M, Upton A, Reid M, Franklin R, et al. Lymphogranuloma venereum in men who have sex with men: evidence of local transmission in New Zealand. $N$ Z Med J. 2015;128(1410):25-9.

52. Bellamy G, Gott M, Hinchliff S. Controversies and contentions: a gay man conducting research with women about their understandings of sexuality, sex and sexual problems. Cult Health Sex. 2011;13(6):699-712.

53. Beltzer N, Saboni L, Sauvage C, Lydie N, Semaille C, Warszawski J. An 18-year follow-up of HIV knowledge, risk perception, and practices in young adults. AIDS. 2013;27(6):1011-9.

54. Bender N, Herrmann B, Andersen B, Hocking JS, van Bergen J, Morgan J, et al. Chlamydia infection, pelvic inflammatory disease, ectopic pregnancy and infertility: cross-national study. Sex Transm Infect. 2011;87(7):601-8.

55. Berg RC. Predictors of never testing for HIV among a national online sample of men who have sex with men in Norway. Scand J Public Health. 2013;41(4):398-404.

56. Bergmann JN, Stockman JK. How does intimate partner violence affect condom and oral contraceptive Use in the United States?: A systematic review of the literature. Contraception. 2015;91(6):438-55.

57. Beymer MR, Weiss RE, Bolan RK, Rudy ET, Bourque LB, Rodriguez JP, et al. Sex on demand: geosocial networking phone apps and risk of sexually transmitted infections among a cross-sectional sample of men who have sex with men in Los Angeles County. Sex Transm Infect. 2014;90(7):567-72. 
58. Beyrer C, Baral SD, van Griensven F, Goodreau SM, Chariyalertsak S, Wirtz AL, et al. Global epidemiology of HIV infection in men who have sex with men. Lancet.

2012;380(9839):367-77.

59. Blake DR, Kearney MH, Oakes JM, Druker SK, Bibace R. Improving participation in Chlamydia screening programs: perspectives of high-risk youth. Arch Pediatr Adolesc Med. 2003;157(6):523-9.

60. Blaxhult A, Granath F, Lidman K, Giesecke J. The influence of age on the latency period to AIDS in people infected by HIV through blood transfusion. AIDS. 1990;4(2):125-9.

61. Boden JM, Fergusson DM, Horwood LJ. Alcohol and STI risk: evidence from a New Zealand longitudinal birth cohort. Drug Alcohol Depend. 2011;113(2-3):200-6.

62. Boily MC, Baggaley RF, Wang L, Masse B, White RG, Hayes RJ, et al. Heterosexual risk of HIV-1 infection per sexual act: systematic review and meta-analysis of observational studies. Lancet Infect Dis. 2009;9(2):118-29.

63. Bosch FX, Lorincz A, Munoz N, Meijer CJ, Shah KV. The causal relation between human papillomavirus and cervical cancer. J Clin Pathol. 2002;55(4):244-65.

64. Bosch FX, Manos MM, Munoz N, Sherman M, Jansen AM, Peto J, et al. Prevalence of human papillomavirus in cervical cancer: a worldwide perspective. International biological study on cervical cancer (IBSCC) Study Group. J Natl Cancer Inst. 1995;87(11):796-802.

65. Boshart M, Gissmann L, Ikenberg H, Kleinheinz A, Scheurlen W, zur Hausen H. A new type of papillomavirus DNA, its presence in genital cancer biopsies and in cell lines derived from cervical cancer. EMBO J. 1984;3(5):1151-7.

66. Bourne A, Reid D, Hickson F, Torres-Rueda S, Weatherburn P. Illicit drug use in sexual settings ('chemsex') and HIV/STI transmission risk behaviour among gay men in South London: findings from a qualitative study. Sex Transm Infect. 2015;91(8):564-8.

67. Boyer CB, Shafer MA, Teitle E, Wibbelsman CJ, Seeberg D, Schachter J. Sexually transmitted diseases in a health maintenance organization teen clinic: associations of race, partner's age, and marijuana use. Arch Pediatr Adolesc Med. 1999;153(8):838-44.

68. Braaten KP, Laufer MR. Human Papillomavirus (HPV), HPV-Related Disease, and the HPV Vaccine. Rev Obstet Gynecol. 2008;1(1):2-10.

69. Brady SS. Lifetime community violence exposure and health risk behavior among young adults in college. J Adolesc Health. 2006;39(4):610-3.

70. Bramley D, Hebert P, Tuzzio L, Chassin M. Disparities in indigenous health: a cross-country comparison between New Zealand and the United States. Am J Public Health. 2005;95(5):844-50.

71. Bremer V, Marcus U, Hamouda O. Syphilis on the rise again in Germany - results from surveillance data for 2011. Euro Surveill. 2012;17(29).

72. Brook MG. Sexually acquired hepatitis. Sex Transm Infect. 2002;78(4):235-40. 
73. Brown DL, Frank JE. Diagnosis and management of syphilis. Am Fam Physician. 2003;68(2):283-90.

74. Brown P, Lyson M, Jenkins T. From diagnosis to social diagnosis. Soc Sci Med. 2011;73(6):939-43.

75. Buisman N, Mow AC, Currie T, Devereux F, Fanning B, Hawkins T, et al. AIDS: knowledge and attitudes in Otago. N Z Med J. 1988;101(845):241-3.

76. Burazeri G, Roshi E, Tavanxhi N. Does knowledge about sexually transmitted infections increase the likelihood of consistent condom use? Prev Med. 2004;39(6):1077-9.

77. Burris S. Stigma, ethics and policy: a commentary on Bayer's "Stigma and the ethics of public health: Not can we but should we". Soc Sci Med. 2008;67(3):473-5; discussion 6-7.

78. Busch MP, Satten GA. Time course of viremia and antibody seroconversion following human immunodeficiency virus exposure. Am J Med. 1997;102(5b):117-24.

79. Bush K, Kivlahan DR, McDonell MB, Fihn SD, Bradley KA. The AUDIT alcohol consumption questions (AUDIT-C): an effective brief screening test for problem drinking. Ambulatory Care Quality Improvement Project (ACQUIP). Alcohol Use Disorders Identification Test. Arch Intern Med. 1998;158(16):1789-95.

80. Butz AM, Gaydos C, Chung SE, Johnson BH, Huettner S, Trent M. Care-Seeking Behavior After Notification Among Young Women With Recurrent Sexually Transmitted Infections After Pelvic Inflammatory Disease. Clin Pediatr (Phila). 2016;55(12):1107-12.

81. Byrne GI. Chlamydia uncloaked. Proc Natl Acad Sci U S A. 2003;100(14):8040-2.

82. Carey MP, Senn TE, Vanable PA, Coury-Doniger P, Urban MA. Do STD clinic patients who consent to sexual health research differ from those who decline? Findings from a randomized controlled trial with implications for the generalization of research results. Sex Transm Dis. 2008;35(1):73-7.

83. Carlin EM, Ziza JM, Keat A, Janier M. 2014 European Guideline on the management of sexually acquired reactive arthritis. Int J STD AIDS. 2014;25(13):901-12.

84. Carter JD, Rehman A, Guthrie JP, Gerard HC, Stanich J, Hudson AP. Attack rate of Chlamydia-induced reactive arthritis and effect of the CCR5-Delta-32 mutation: a prospective analysis. J Rheumatol. 2013;40(9):1578-82.

85. Cassell JA, Brook MG, Mercer CH, Murphy S, Johnson AM. Treating sexually transmitted infections in primary care: a missed opportunity? Sex Transm Infect. 2003;79(2):134-6.

86. Catchpole M, Connor N, Brady A, Kinghorn G, Mercey D, Band B, et al. Behavioural and demographic characteristics of attenders at two genitourinary medicine clinics in England. Genitourin Med. 1997;73(6):457-61.

87. Catterall RD. Incidence of chronic genital infection in male patients with uveitis. A preliminary report. Br J Vener Dis. 1958;34(4):254-5. 
88. Centers for Disease Control and Prevention. Antibiotic Resistance Threats in the United States. CDC, 2013a.

89. Centers for Disease Control and Prevention. Establishing a Holistic Framework to Reduce Inequities in HIV, Viral Hepatitis, STDs, and Tuberculosis in the United States. Atlanta (GA), U.S.: Department of Health and Human Services, Centers for Disease Control and Prevention, 2010.

90. Centers for Disease Control and Prevention. How Tuskegee Changed Research Practices (Webpage). Available at http://www.cdc.gov/tuskegee/after.htm [Accessed 03/12/16]. 2013b.

91. Centers for Disease Control and Prevention. Sexually Transmitted Disease Surveillance 2014. Atlanta: U.S. Department of Health and Human Services: 2015.

92. Centers for Disease Control and Prevention. Syphilis - CDC Fact Sheet. Available at: http://www.cdc.gov/std/syphilis/stdfact-syphilis.htm [Accessed 01/12/16]. 2014.

93. Centers of Disease Control and Prevention. Case Counts - 2014 Ebola Outbreak in West Africa. (Updated April 13, 2016) Available at http://www.cdc.gov/vhf/ebola/outbreaks/2014west-africa/case-counts.html [Accessed 01/12/16]. 2016.

94. Centre for Reviews and Dissemination. Systematic Reviews: CRD's guidance for undertaking reviews in health care. University of York: 2009 Contract No.: ISBN 978-1-900640-47-3.

95. Chaillet P, Tayler-Smith K, Zachariah R, Duclos N, Moctar D, Beelaert G, et al. Evaluation of four rapid tests for diagnosis and differentiation of HIV-1 and HIV-2 infections in GuineaConakry, West Africa. Trans R Soc Trop Med Hyg. 2010;104(9):571-6.

96. Chanakira E, Goyder E, Freeman J, O'Cathain A, Kinghorn G, Jakubovic M. Social and psychosocial factors associated with high risk sexual behaviour among university students in the United Kingdom: a web-survey. Int J STD AIDS. 2014;26(6):369-78.

97. Charmaz K. Premises, principles, and practices in qualitative research: revisiting the foundations. Qual Health Res. 2004;14(7):976-93.

98. Chaudhary R, Heffernan CM, Illsley AL, Jarvie LK, Lattimer C, Nwuba AE, et al. Opportunistic screening for Chlamydia: a pilot study into male perspectives on provision of Chlamydia screening in a UK university. J Public Health (Oxf). 2008;30(4):466-71.

99. Chelimo C, Wouldes TA. Human papillomavirus knowledge and awareness among undergraduates in healthcare training in New Zealand. N Z Med J. 2009;122(1304):33-45.

100. Chernesky MA. The evaluation of diagnostic tests for sexually transmitted infections. Can J Infect Dis Med Microbiol. 2005;16(2):80-2.

101. Chetwynd J. Knowledge about HIV/AIDS amongst the New Zealand public: 1987-9. N Z Med J. 1991;104(923):473-5.

102. Chinsembu K. Sexually Transmitted Infections in Adolescents. The Open Infectious Diseases Journal. 2009;3:107-17. 
103. Choi KH, Zheng X, Zhou H, Chen W, Mandel J. Treatment delay and reliance on private physicians among patients with sexually transmitted diseases in China. Int J STD AIDS. 1999; 10(5):309-15.

104. Chow EP, Camilleri S, Ward C, Huffam S, Chen MY, Bradshaw CS, et al. Duration of gonorrhoea and chlamydia infection at the pharynx and rectum among men who have sex with men: a systematic review. Sex Health. 2016;13(3):199-204.

105. Christie A, Davies-Wayne GJ, Cordier-Lassalle T, Blackley DJ, Laney AS, Williams DE, et al. Possible sexual transmission of Ebola virus - Liberia, 2015. MMWR Morb Mortal Wkly Rep. 2015;64(17):479-81.

106. Christodoulides M. Neisseria proteomics for antigen discovery and vaccine development. Expert Rev Proteomics. 2014;11(5):573-91.

107. Church A. Estimating the effect of incentives on mail survey response rates: a meta-analysis. Public Opin Q. 1993;57(1):62-79.

108. Cochran SD, Mays VM. Applying Social Psychological Models to Predicting HIV-Related Sexual Risk Behaviors Among African Americans. J Black Psychol. 1993;19(2):142-54.

109. Cole K, Mitchell D, Leighton J, Aitken J, Mackay J. Determination of Chlamydia trachomatis prevalence in asymptomatic male military personnel in New Zealand: comparing performances of COBAS Amplicor and Abbott LCx Chlamydia detection systems for urine specimens. New Zealand Journal of Medical Laboratory Science. 2001;33(55):71-4.

110. Cole MJ, Spiteri G, Chisholm SA, Hoffmann S, Ison CA, Unemo M, et al. Emerging cephalosporin and multidrug-resistant gonorrhoea in Europe. Euro Surveill. 2014;19(45):20955.

111. Coles VA, Patel AS, Allen FL, Keeping ST, Carroll SM. The association of human papillomavirus vaccination with sexual behaviours and human papillomavirus knowledge: a systematic review. Int J STD AIDS. 2015;26(11):777-88.

112. Collantes E, Zarco P, Munoz E, Juanola X, Mulero J, Fernandez-Sueiro JL, et al. Disease pattern of spondyloarthropathies in Spain: description of the first national registry (REGISPONSER) extended report. Rheumatology. 2007;46(8):1309-15.

113. Connor J, Paul C, Sharples K, Dickson N. Patterns of disease and HIV testing at sexually transmitted disease clinics. N Z Med J. 1997;110(1057):452-5.

114. Connor J, Psutka R, Cousins K, Gray A, Kypri K. Risky drinking, risky sex: a national study of New Zealand university students. Alcohol Clin Exp Res. 2013;37(11):1971-8.

115. Connor JL, Kydd RM, Dickson NP. Alcohol Involvement in Sexual Behaviour and Adverse Sexual Health Outcomes from 26 to 38 Years of Age. PLoS One. 2015;10(8):e0135660.

116. Cook C. Diagnostic classification, viral sexually transmitted infections and discourses of femininity: limits of normalisation to erase stigma. Nurs Inq. 2013;20(2):145-55. 
117. Cook RL, Clark DB. Is there an association between alcohol consumption and sexually transmitted diseases? A systematic review. Sex Transm Dis. 2005;32(3):156-64.

118. Cook RL, Hutchison SL, Ostergaard L, Braithwaite RS, Ness RB. Systematic review: noninvasive testing for Chlamydia trachomatis and Neisseria gonorrhoeae. Ann Intern Med. 2005;142(11):914-25.

119. Cooper ML. Alcohol use and risky sexual behavior among college students and youth: evaluating the evidence. J Stud Alcohol Suppl. 2002(14):101-17.

120. Cormack D, Robson, C. Classification and output of multiple ethnicities: considerations for monitoring Māori Health. Wellington: Te Rōpū Rangahau Hauora a Eru Pōmare: 2010.

121. Cornally N, McCarthy G. Help-seeking behaviour: a concept analysis. Int J Nurs Pract. 2011;17(3):280-8.

122. Coyne IT. Sampling in qualitative research. Purposeful and theoretical sampling; merging or clear boundaries? J Adv Nurs. 1997;26(3):623-30.

123. Cragg A, Steenbeek A, Asbridge M, Andreou P, Langille D. Sexually transmitted infection testing among heterosexual Maritime Canadian university students engaging in different levels of sexual risk taking. Can J Public Health. 2016;107(2):e149-54.

124. Creswell JW. A Concise Introduction to Mixed Methods Research. California, U.S: Sage publishing; 2015.

125. Creswell JW, Fetters MD, Ivankova NV. Designing a mixed methods study in primary care. Ann Fam Med. 2004;2(1):7-12.

126. Crichton J, Hickman M, Campbell R, Batista-Ferrer H, Macleod J. Socioeconomic factors and other sources of variation in the prevalence of genital chlamydia infections: A systematic review and meta-analysis. BMC Public Health. 2015;15:729.

127. Crosby RA, Newman D, Kamb ML, Zenilman J, Douglas J, Iatesta M. Misconceptions about STD-protective behavior. Project RESPECT Study Group. Am J Prev Med. 2000;19(3):16773.

128. Cruciani M, Mengoli C, Malena M, Serpelloni G, Parisi SG, Moyle G, et al. Virological efficacy of abacavir: systematic review and meta-analysis. J Antimicrob Chemother. 2014;69(12):3169-80.

129. Cuffe KM, Newton-Levinson A, Gift TL, McFarlane M, Leichliter JS. Sexually Transmitted Infection Testing Among Adolescents and Young Adults in the United States. J Adolesc Health. 2016;58(5):512-9.

130. Cunningham SD, Kerrigan D, Pillay KB, Ellen JM. Understanding the role of perceived severity in STD-related care-seeking delays. J Adolesc Health. 2005;37(1):69-74.

131. Cunningham SD, Kerrigan DL, Jennings JM, Ellen JM. Relationships between perceived STD-related stigma, STD-related shame and STD screening among a household sample of adolescents. Perspect Sex Reprod Health. 2009;41(4):225-30. 
132. Currie MJ, Schmidt M, Davis BK, Baynes AM, O'Keefe EJ, Bavinton TP, et al. 'Show me the money': financial incentives increase chlamydia screening rates among tertiary students: a pilot study. Sex Health. 2010;7(1):60-5.

133. Curtis AH. A cause of adhesions in the right upper quadrant. JAMA. 1930;94:1221-2.

134. Cutts FT, Franceschi S, Goldie S, Castellsague X, de Sanjose S, Garnett G, et al. Human papillomavirus and HPV vaccines: a review. Bull World Health Organ. 2007;85(9):719-26.

135. D'Ortenzio E, Matheron S, Yazdanpanah Y, de Lamballerie X, Hubert B, Piorkowski G, et al. Evidence of Sexual Transmission of Zika Virus. N Engl J Med. 2016;374(22):2195-8.

136. Darroch J, Myers L, Cassell J. Sex differences in the experience of testing positive for genital chlamydia infection: a qualitative study with implications for public health and for a national screening programme. Sex Transm Infect. 2003;79(5):372-3.

137. Davies B, Anderson SJ, Turner KM, Ward H. How robust are the natural history parameters used in chlamydia transmission dynamic models? A systematic review. Theor Biol Med Model. 2014a;11:8.

138. Davies B, Turner KM, Frolund M, Ward H, May MT, Rasmussen S, et al. Risk of reproductive complications following chlamydia testing: a population-based retrospective cohort study in Denmark. Lancet Infect Dis. 2016;16(9):1057-64.

139. Davies B, Ward H, Leung S, Turner KM, Garnett GP, Blanchard JF, et al. Heterogeneity in risk of pelvic inflammatory diseases after chlamydia infection: a population-based study in Manitoba, Canada. J Infect Dis. 2014b;210 Suppl 2:S549-55.

140. Davies J, McCrae BP, Frank J, Dochnahl A, Pickering T, Harrison B, et al. Identifying male college students' perceived health needs, barriers to seeking help, and recommendations to help men adopt healthier lifestyles. J Am Coll Health. 2000;48(6):259-67.

141. de Visser R, Smith A, Richters J. Can we generalise to other young people from studies of sexual risk behaviour among university students? Aust N Z J Public Health. 2005;29(5):43641.

142. Deckard DT, Chung WM, Brooks JT, Smith JC, Woldai S, Hennessey M, et al. Male-to-Male Sexual Transmission of Zika Virus - Texas, January 2016. MMWR Morb Mortal Wkly Rep. 2016;65(14):372-4.

143. Deen GF, Knust B, Broutet N, Sesay FR, Formenty P, Ross C, et al. Ebola RNA Persistence in Semen of Ebola Virus Disease Survivors - Preliminary Report. N Engl J Med. 2015;[Epub ahead of print].

144. Deogan C, Cnattingius S, Mansdotter A. Risk of self-reported Chlamydia trachomatis infection by social and lifestyle factors: a study based on survey data from young adults in Stockholm, Sweden. Eur J Contracept Reprod Health Care. 2012;17(6):458-67.

145. Department of Health. Alcohol Guidelines Review - Report from the Guidelines development group to the UK Chief Medical Officers. Department of Health, 2016. 
146. Deren S, Stephens R, Davis WR, Feucht TE, Tortu S. The impact of providing incentives for attendance at AIDS prevention sessions. Public Health Rep. 1994;109(4):548-54.

147. Detels R, Green AM, Klausner JD, Katzenstein D, Gaydos C, Handsfield H, et al. The incidence and correlates of symptomatic and asymptomatic Chlamydia trachomatis and Neisseria gonorrhoeae infections in selected populations in five countries. Sex Transm Dis. 2011;38(6):503-9.

148. Devonshire P, Hillman R, Capewell S, Clark BJ. Knowledge of Chlamydia trachomatis genital infection and its consequences in people attending a genitourinary medicine clinic. Sex Transm Infect. 1999;75(6):409-11.

149. Diallo B, Sissoko D, Loman NJ, Bah HA, Bah H, Worrell MC, et al. Resurgence of Ebola Virus Disease in Guinea Linked to a Survivor With Virus Persistence in Seminal Fluid for More Than 500 Days. Clin Infect Dis. 2016;63(10):1353-6.

150. Dick GW, Kitchen SF, Haddow AJ. Zika virus. I. Isolations and serological specificity. Trans $R$ Soc Trop Med Hyg. 1952;46(5):509-20.

151. Dickson N, Ludlam A, Saxton P, Hughes A. Self-reported STIs and sexual health checks in a cross-sectional study of gay and bisexual men in New Zealand. Sex Transm Infect. 2015;91(1):49-54.

152. Dickson N, Paul C, Herbison P. Where young people with multiple sexual partners seek medical care: implications for screening for chlamydial infection. Sex Transm Infect. 1998;74(6):445-7.

153. DiClemente RJ, Salazar LF, Crosby RA. A review of STD/HIV preventive interventions for adolescents: sustaining effects using an ecological approach. J Pediatr Psychol. 2007;32(8):888-906.

154. Diclemente RJ, Wingood GM, Sionean C, Crosby R, Harrington K, Davies S, et al. Association of adolescents' history of sexually transmitted disease (STD) and their current high-risk behavior and STD status: a case for intensifying clinic-based prevention efforts. Sex Transm Dis. 2002;29(9):503-9.

155. Dielissen PW, Teunissen DA, Lagro-Janssen AL. Chlamydia prevalence in the general population: is there a sex difference? a systematic review. BMC Infect Dis. 2013;13:534.

156. Dietz K. The estimation of the basic reproduction number for infectious diseases. Stat Methods Med Res. 1993;2(1):23-41.

157. Dimmitt Champion J, Harlin B, Collins JL. Sexual risk behavior and STI health literacy among ethnic minority adolescent women. Appl Nurs Res. 2013;26(4):204-9.

158. Domeika M, Oscarsson L, Hallen A, Hjelm E, Sylvan S. Mailed urine samples are not an effective screening approach for Chlamydia trachomatis case finding among young men. $J$ Eur Acad Dermatol Venereol. 2007;21(6):789-94.

159. Dong Y, Peng CY. Principled missing data methods for researchers. Springerplus. 2013;2(1):222. 
160. Dougados M, Baeten D. Spondyloarthritis. Lancet. 2011;377(9783):2127-37.

161. Dougados M, van der Linden S, Juhlin R, Huitfeldt B, Amor B, Calin A, et al. The European Spondylarthropathy Study Group preliminary criteria for the classification of spondylarthropathy. Arthritis Rheum. 1991;34(10):1218-27.

162. Doyle DM, Molix L. Social Stigma and Sexual Minorities' Romantic Relationship Functioning: A Meta-Analytic Review. Pers Soc Psychol Bull. 2015;41(10):1363-81.

163. Dukers-Muijrers NH, Morre SA, Speksnijder A, van der Sande MA, Hoebe CJ. Chlamydia trachomatis test-of-cure cannot be based on a single highly sensitive laboratory test taken at least 3 weeks after treatment. PLoS One. 2012;7(3):e34108.

164. Duncan B, Hart G. Sexuality and health: the hidden costs of screening for Chlamydia trachomatis. BMJ. 1999;318(7188):931-3.

165. Duncan D, Bergen MB. Knowledge of New Zealand youth regarding sexuality and AIDS. $J$ Sex Marital Ther. 1997;23(1):47-51.

166. Dunne EF, Nielson CM, Stone KM, Markowitz LE, Giuliano AR. Prevalence of HPV infection among men: A systematic review of the literature. J Infect Dis. 2006;194(8):104457.

167. Dunne EF, Unger ER, Sternberg M, McQuillan G, Swan DC, Patel SS, et al. Prevalence of HPV infection among females in the United States. JAMA. 2007;297(8):813-9.

168. Durst M, Gissmann L, Ikenberg H, zur Hausen H. A papillomavirus DNA from a cervical carcinoma and its prevalence in cancer biopsy samples from different geographic regions. Proc Natl Acad Sci U S A. 1983;80(12):3812-5.

169. Eastman-Mueller H, Carr D, Osterlind S. Sexual health survey. In: Fisher TD, Davis CM, Yarber W, Davis SL, editors. Handbook of sexuality-related measures, 3rd edn. Danvers, MA: Taylor and Francis Publishing; 2010. p. 340-44.

170. Ebola virus infections. Br Med J. 1977;2(6086):539-40.

171. Eickhoff CA, Decker CF. Syphilis. Dis Mon. 2016;62(8):280-6.

172. Ekeroma AJ, Pandit L, Bartley C, Ikenasio-Thorpe B, Thompson JM. Screening for sexually transmitted infections in pregnancy at Middlemore Hospital, 2009. N Z Med J. 2012;125(1359):23-9.

173. Ekstrand M, Engblom C, Larsson M, Tyden T. Sex education in Swedish schools as described by young women. Eur J Contracept Reprod Health Care. 2011;16(3):210-24.

174. Eley A, Hosseinzadeh S, Hakimi H, Geary I, Pacey AA. Apoptosis of ejaculated human sperm is induced by co-incubation with Chlamydia trachomatis lipopolysaccharide. Hum Reprod. 2005a;20(9):2601-7.

175. Eley A, Pacey AA, Galdiero M, Galdiero M, Galdiero F. Can Chlamydia trachomatis directly damage your sperm? Lancet Infect Dis. 2005b;5(1):53-7. 
176. Ellis S, Grey A. Prevention of Sexually Transmitted Infections (STIs): A Review of Reviews into the Effectiveness of Non-Clinical Interventions. London: Health Development Agency, 2004.

177. Espinoza LR, Garcia-Valladares I. Of bugs and joints: the relationship between infection and joints. Reumatol Clin. 2013;9(4):229-38.

178. ESR Annual Surveillance Report - Sexually Transmitted Infections in New Zealand, 2013. Porirua, New Zealand: The Institute of Environmental Science and Research Ltd., 2014.

179. ESR Annual Surveillance Report - Sexually Transmitted Infections in New Zealand, 2014. Porirua, New Zealand: The Institute of Environmental Science and Research Ltd, 2015.

180. Ethier KA, Kershaw T, Niccolai L, Lewis JB, Ickovics JR. Adolescent women underestimate their susceptibility to sexually transmitted infections. Sex Transm Infect. 2003;79(5):408-11.

181. European Centre for Disease Prevention and Control. Chlamydia control in Europe: a survey of Member States. Stockholm: ECDC, 2014.

182. European Centre for Disease Prevention and Control. Guidance on chlamydia control in Europe 2015. Stockholm: ECDC, 2016.

183. Ewert C, Collyer A, Temple-Smith M. 'Most young men think you have to be naked in front of the GP': a qualitative study of male university students' views on barriers to sexual health. Sexual Health. 2016;13(2):124-30.

184. Fagan P, McDonell P. Knowledge, attitudes and behaviours in relation to safe sex, sexually transmitted infections (STI) and HIV/AIDS among remote living north Queensland youth. Aust N Z J Public Health. 2010;34 Suppl 1:S52-6.

185. Fairley CK, Chen S, Tabrizi SN, Quinn MA, McNeil JJ, Garland SM. Tampons: a novel patient-administered method for the assessment of genital human papillomavirus infection. $J$ Infect Dis. 1992;165(6):1103-6.

186. Fajardo-Bernal L, Aponte-Gonzalez J, Vigil P, Angel-Muller E, Rincon C, Gaitan HG, et al. Home-based versus clinic-based specimen collection in the management of Chlamydia trachomatis and Neisseria gonorrhoeae infections. Cochrane Database Syst Rev. 2015;9:Cd011317.

187. Fatahzadeh M, Schwartz RA. Human herpes simplex virus infections: epidemiology, pathogenesis, symptomatology, diagnosis, and management. J Am Acad Dermatol. 2007;57(5):737-63.

188. Fatusi A, Wang W. Multiple sexual partnership mediates the association between early sexual debut and sexually transmitted infection among adolescent and young adult males in Nigeria. Eur J Contracept Reprod Health Care. 2009;14(2):134-43.

189. Faxelid E, Ahlberg BM, Ndulo J, Krantz I. Health-seeking behaviour of patients with sexually transmitted diseases in Zambia. East Afr Med J. 1998;75(4):232-6. 
190. Faxelid E, Ndulo J, Ahlberg BM, Krantz I. Behaviour, knowledge and reactions concerning sexually transmitted diseases: implications for partner notification in Lusaka. East Afr Med J. 1994;71(2):118-21.

191. Fennell J. "And Isn't that the point?": pleasure and contraceptive decisions. Contraception. 2014;89(4):264-70.

192. Fenton KA, Johnson AM, McManus S, Erens B. Measuring sexual behaviour: methodological challenges in survey research. Sex Transm Infect. 2001a;77(2):84-92.

193. Fenton KA, Korovessis C, Johnson AM, McCadden A, McManus S, Wellings K, et al. Sexual behaviour in Britain: reported sexually transmitted infections and prevalent genital Chlamydia trachomatis infection. Lancet. 2001b;358(9296):1851-4.

194. Fenton KA, Mercer CH, Johnson AM, Byron CL, McManus S, Erens B, et al. Reported sexually transmitted disease clinic attendance and sexually transmitted infections in Britain: prevalence, risk factors, and proportionate population burden. J Infect Dis. 2005a;191 Suppl $1:$ S127-38.

195. Fenton KA, Mercer CH, McManus S, Erens B, Wellings K, Macdowall W, et al. Ethnic variations in sexual behaviour in Great Britain and risk of sexually transmitted infections: a probability survey. Lancet. 2005b;365(9466):1246-55.

196. Fergusson DM, Boden JM, Horwood LJ. Circumcision status and risk of sexually transmitted infection in young adult males: an analysis of a longitudinal birth cohort. Pediatrics. 2006;118(5):1971-7.

197. Fergusson DM, Lynskey MT, Horwood LJ. AIDS knowledge and condom use in a birth cohort of 16 year olds. N Z Med J. 1994;107(990):480-3.

198. Fetters MD, Curry LA, Creswell JW. Achieving integration in mixed methods designsprinciples and practices. Health Serv Res. 2013;48(6 Pt 2):2134-56.

199. Fifer H, Ison CA. Nucleic acid amplification tests for the diagnosis of Neisseria gonorrhoeae in low-prevalence settings: a review of the evidence. Sex Transm Infect. 2014;90(8):577-9.

200. Fischer WA, 2nd, Wohl DA. Confronting Ebola as a Sexually Transmitted Infection. Clin Infect Dis. 2016;62(10):1272-6.

201. Fitz-Hugh T. Acute gonococcic peritonitis of the right upper quadrant. JAMA. 1934;102:2094-6.

202. Foley E, Patel R. Destigmatising STIs: remaining challenges, new opportunities. Sex Transm Infect. 2001;77(5):306-7.

203. Fonck K, Mwai C, Ndinya-Achola J, Bwayo J, Temmerman M. Health-seeking and sexual behaviors among primary healthcare patients in Nairobi, Kenya. Sex Transm Dis.

2002;29(2):106-11. 
204. Fonck K, Mwai C, Rakwar J, Kirui P, Ndinya-Achola JO, Temmerman M. Healthcareseeking behavior and sexual behavior of patients with sexually transmitted diseases in Nairobi, Kenya. Sex Transm Dis. 2001;28(7):367-71.

205. Forrest DW, Metsch LR, LaLota M, Cardenas G, Beck DW, Jeanty Y. Crystal methamphetamine use and sexual risk behaviors among HIV-positive and HIV-negative men who have sex with men in South Florida. J Urban Health. 2010;87(3):480-5.

206. Fortenberry JD. Health care seeking behaviors related to sexually transmitted diseases among adolescents. Am J Public Health. 1997;87(3):417-20.

207. Fortenberry JD, McFarlane M, Bleakley A, Bull S, Fishbein M, Grimley DM, et al. Relationships of stigma and shame to gonorrhea and HIV screening. Am J Public Health. 2002;92(3):378-81.

208. Fortenberry JD, McFarlane MM, Hennessy M, Bull SS, Grimley DM, St Lawrence J, et al. Relation of health literacy to gonorrhoea related care. Sex Transm Infect. 2001;77(3):206-11.

209. Foster LR, Byers ES. Predictors of stigma and shame related to sexually transmitted infections: Attitudes, education, and knowledge. Can J Hum Sex. 2008;17(4):193-202.

210. Foy BD, Kobylinski KC, Chilson Foy JL, Blitvich BJ, Travassos da Rosa A, Haddow AD, et al. Probable non-vector-borne transmission of Zika virus, Colorado, USA. Emerg Infect Dis. 2011;17(5):880-2.

211. Frith J. Syphilis - Its Early History and Treatment Until Penicillin, and the Debate on its Origins. J Mil Veterans Health. 2012;20(4):49-58.

212. Fryar CD, Hirsch R, Porter KS, Kottiri B, Brody DJ, Louis T. Drug use and sexual behaviors reported by adults: United States, 1999-2002. Adv Data. 2007(384):1-14.

213. Fuzzell L, Fedesco HN, Alexander SC, Fortenberry JD, Shields CG. "I just think that doctors need to ask more questions": Sexual minority and majority adolescents' experiences talking about sexuality with healthcare providers. Patient Educ Couns. 2016;99(9):1467-72.

214. Gail MH, Benichou J. Encyclopedia of Epidemiologic Methods. Armitage P, Colton T, editors: Wiley; 2000.

215. Galea S, Tracy M. Participation rates in epidemiologic studies. Ann Epidemiol. 2007;17(9):643-53.

216. Gallo MF, Kilbourne-Brook M, Coffey PS. A review of the effectiveness and acceptability of the female condom for dual protection. Sex Health. 2012;9(1):18-26.

217. Ganser-Pornillos BK, Yeager M, Pornillos O. Assembly and architecture of HIV. Adv Exp Med Biol. 2012;726:441-65.

218. Gao D, Lou Y, He D, Porco TC, Kuang Y, Chowell G, et al. Prevention and Control of Zika as a Mosquito-Borne and Sexually Transmitted Disease: A Mathematical Modeling Analysis. Sci Rep. 2016;6:28070. 
219. Garland SM, Steben M. Genital herpes. Best Pract Res Clin Obstet Gynaecol. 2014;28(7):1098-110.

220. Garnett GP. The geographical and temporal evolution of sexually transmitted disease epidemics. Sex Transm Infect. 2002;78 Suppl 1:i14-9.

221. Geisler WM, Chyu L, Kusunoki Y, Upchurch DM, Hook EW, 3rd. Health insurance coverage, health care-seeking behaviors, and genital chlamydial infection prevalence in sexually active young adults. Sex Transm Dis. 2006;33(6):389-96.

222. Geisler WM, Wang C, Morrison SG, Black CM, Bandea CI, Hook EW, 3rd. The natural history of untreated Chlamydia trachomatis infection in the interval between screening and returning for treatment. Sex Transm Dis. 2008;35(2):119-23.

223. Gencay M, Koskiniemi M, Ammala P, Fellman V, Narvanen A, Wahlstrom T, et al. Chlamydia trachomatis seropositivity is associated both with stillbirth and preterm delivery. Apmis. 2000;108(9):584-8.

224. Gerbase AC, Rowley JT, Heymann DH, Berkley SF, Piot P. Global prevalence and incidence estimates of selected curable STDs. Sex Transm Infect. 1998;74 Suppl 1:S12-6.

225. Gerressu M, Mercer CH, Cassell JA, Brook G, Dave S. The importance of distinguishing between black Caribbeans and Africans in understanding sexual risk and care-seeking behaviours for sexually transmitted infections: evidence from a large survey of people attending genitourinary medicine clinics in England. J Public Health (Oxf). 2012;34(3):41120.

226. Gerver SM, Easterbrook PJ, Anderson M, Solarin I, Elam G, Fenton KA, et al. Sexual risk behaviours and sexual health outcomes among heterosexual black Caribbeans: comparing sexually transmitted infection clinic attendees and national probability survey respondents. Int J STD AIDS. 2011;22(2):85-90.

227. Gesink DC, Sullivan AB, Miller WC, Bernstein KT. Sexually transmitted disease core theory: roles of person, place, and time. Am J Epidemiol. 2011;174(1):81-9.

228. Giakoumelou S, Wheelhouse N, Cuschieri K, Entrican G, Howie SE, Horne AW. The role of infection in miscarriage. Hum Reprod Update. 2016;22(1):116-33.

229. Gibney KB, O'Toole J, Sinclair M, Leder K. Disease burden of selected gastrointestinal pathogens in Australia, 2010. Int J Infect Dis. 2014;28:176-85.

230. Gilmore N, Somerville MA. Stigmatization, scapegoating and discrimination in sexually transmitted diseases: overcoming 'them' and 'us'. Soc Sci Med. 1994;39(9):1339-58.

231. Gimenes F, Souza RP, Bento JC, Teixeira JJ, Maria-Engler SS, Bonini MG, et al. Male infertility: a public health issue caused by sexually transmitted pathogens. Nat Rev Urol. 2014;11(12):672-87.

232. Girard MP, Osmanov S, Assossou OM, Kieny MP. Human immunodeficiency virus (HIV) immunopathogenesis and vaccine development: a review. Vaccine. 2011;29(37):6191-218. 
233. Glick SN, Morris M, Foxman B, Aral SO, Manhart LE, Holmes KK, et al. A comparison of sexual behavior patterns among men who have sex with men and heterosexual men and women. J Acquir Immune Defic Syndr. 2012;60(1):83-90.

234. Gobin M, Verlander N, Maurici C, Bone A, Nardone A. Do sexual health campaigns work? An outcome evaluation of a media campaign to increase chlamydia testing among young people aged 15-24 in England. BMC Public Health. 2013;13:484.

235. Goffman E. Stigma: Notes on the Management of Spoiled Identity,1963.

236. Goldenberg RL, McClure EM, Saleem S, Reddy UM. Infection-related stillbirths. Lancet. 2010;375(9724):1482-90.

237. Gonzales GF, Munoz G, Sanchez R, Henkel R, Gallegos-Avila G, Diaz-Gutierrez O, et al. Update on the impact of Chlamydia trachomatis infection on male fertility. Andrologia. 2004;36(1):1-23.

238. Gott CM, Rogstad KE, Riley V, Ahmed-Jushuf I. Delay in symptom presentation among a sample of older GUM clinic attenders. Int J STD AIDS. 1999;10(1):43-6.

239. Gotz HM, Veldhuijzen IK, van Bergen JE, Hoebe CJ, de Zwart O, Richardus JH, et al. Acceptability and consequences of screening for chlamydia trachomatis by home-based urine testing. Sex Transm Dis. 2005;32(9):557-62.

240. Graham S, Guy RJ, Donovan B, McManus H, Su JY, El-Hayek C, et al. Epidemiology of chlamydia and gonorrhoea among Indigenous and non-Indigenous Australians, 2000-2009. Med J Aust. 2012;197(11):642-6.

241. Graham S, Guy RJ, Wand HC, Kaldor JM, Donovan B, Knox J, et al. A sexual health quality improvement program (SHIMMER) triples chlamydia and gonorrhoea testing rates among young people attending Aboriginal primary health care services in Australia. BMC Infect Dis. 2015;15:370.

242. Graseck AS, Shih SL, Peipert JF. Home versus clinic-based specimen collection for Chlamydia trachomatis and Neisseria gonorrhoeae. Expert Rev Anti Infect Ther. 2011;9(2):183-94.

243. Greaves A, Lonsdale S, Whinney S, Hood E, Mossop H, Olowokure B. University undergraduates' knowledge of chlamydia screening services and chlamydia infection following the introduction of a National Chlamydia Screening Programme. Eur J Contracept Reprod Health Care. 2009;14(1):61-8.

244. Green J. Qualitative methods for health research. London; California; New Delhi: SAGE Publications; 2009.

245. Grulich AE, de Visser RO, Badcock PB, Smith AM, Richters J, Rissel C, et al. Knowledge about and experience of sexually transmissible infections in a representative sample of adults: the Second Australian Study of Health and Relationships. Sex Health. 2014;11(5):481-94.

246. Guidelines for Cervical Screening in New Zealand. Wellington: The National Screening Unit, Ministry of Health, 2008. 
247. Gupta GR, Parkhurst JO, Ogden JA, Aggleton P, Mahal A. Structural approaches to HIV prevention. Lancet. 2008;372(9640):764-75.

248. Hafner LM, Wilson DP, Timms P. Development status and future prospects for a vaccine against Chlamydia trachomatis infection. Vaccine. 2014;32(14):1563-71.

249. Haggerty CL, Gottlieb SL, Taylor BD, Low N, Xu F, Ness RB. Risk of sequelae after Chlamydia trachomatis genital infection in women. J Infect Dis. 2010;201 Suppl 2:S134-55.

250. Hall NM, Peterson J, Johnson M. To Test or Not to Test: Barriers and Solutions to Testing African American College Students for HIV at a Historically Black College/University. $J$ Health Dispar Res Pract. 2014;7(1):2.

251. Hamdulay SS, Glynne SJ, Keat A. When is arthritis reactive? Postgrad Med J. 2006;82(969):446-53.

252. Hannu T, Inman R, Granfors K, Leirisalo-Repo M. Reactive arthritis or post-infectious arthritis? Best Pract Res Clin Rheumatol. 2006;20(3):419-33.

253. Harper DM, Franco EL, Wheeler C, Ferris DG, Jenkins D, Schuind A, et al. Efficacy of a bivalent $\mathrm{L} 1$ virus-like particle vaccine in prevention of infection with human papillomavirus types 16 and 18 in young women: a randomised controlled trial. Lancet. 2004;364(9447):1757-65.

254. Harper DM, Franco EL, Wheeler CM, Moscicki AB, Romanowski B, Roteli-Martins CM, et al. Sustained efficacy up to 4.5 years of a bivalent L1 virus-like particle vaccine against human papillomavirus types 16 and 18: follow-up from a randomised control trial. Lancet. 2006;367(9518):1247-55.

255. Hartney T, Baraitser P, Nardone A. Self-Reported Impact of Chlamydia Testing on Subsequent Behavior: Results of an Online Survey of Young Adults in England. Sex Transm Dis. 2015;42(9):486-91.

256. Hay PE, Kerry SR, Normansell R, Horner PJ, Reid F, Kerry SM, et al. Which sexually active young female students are most at risk of pelvic inflammatory disease? A prospective study. Sex Transm Infect. 2016;92(1):63-6.

257. Health Protection Report. Recent epidemiology of infectious syphilis and congenital syphilis. Vol 7 No. 44. Public Health England, 2013.

258. Health Protection Report. Sexually transmitted infections and chlamydia screening in England, 2015. Vol 10 No. 22. Public Health England, 2016.

259. Healthy People 2010. Sexually Transmitted Diseases. 2nd edition. Washington, DC: U.S. Department of Health and Human Services, 2000.

260. Hegazi A, Lee MJ, Whittaker W, Green S, Simms R, Cutts R, et al. Chemsex and the city: sexualised substance use in gay bisexual and other men who have sex with men attending sexual health clinics. Int J STD AIDS. 2016; [Epub ahead of print]. 
261. Henning D, Alice R, Sanci L, Dunning T. Screening for chlamydia trachomatis: barriers for homeless young people. Aust J Adv Nurs. 2007;24(3):8-13.

262. Henrickson M, Dickson N, Mhlanga F, Ludlam A. Stigma, lack of knowledge and prevalence maintain HIV risk among Black Africans in New Zealand. Aust N Z J Public Health. 2015;39(1):32-7.

263. Heritage J, Jones M. A study of young peoples' attitudes to opportunistic Chlamydia testing in UK general practice. Reprod Health. 2008;5:11.

264. Hernandez BY, Wilkens LR, Zhu X, Thompson P, McDuffie K, Shvetsov YB, et al. Transmission of human papillomavirus in heterosexual couples. Emerg Infect Dis. 2008;14(6):888-94.

265. Heymann DL. Control of communicable diseases manual. 19th ed. Washington, DC: American Public Health Association; 2008.

266. Higgins JA, Wang Y. The Role of Young Adults' Pleasure Attitudes in Shaping Condom Use. Am J Public Health. 2015;105(7):1329-32.

267. Hillis SD, Joesoef R, Marchbanks PA, Wasserheit JN, Cates W, Jr., Westrom L. Delayed care of pelvic inflammatory disease as a risk factor for impaired fertility. Am J Obstet Gynecol. 1993;168(5):1503-9.

268. Hitchcock P. Scientific Evidence on Condom Effectiveness for Sexually Transmitted Disease (STD) Prevention: workshop summary. Herndon, USA: 2001.

269. Hjelmevoll SO, Olsen ME, Sollid JU, Haaheim H, Melby KK, Moi H, et al. Appropriate time for test-of-cure when diagnosing gonorrhoea with a nucleic acid amplification test. Acta Derm Venereol. 2012;92(3):316-9.

270. Ho GY, Bierman R, Beardsley L, Chang CJ, Burk RD. Natural history of cervicovaginal papillomavirus infection in young women. N Engl J Med. 1998;338(7):423-8.

271. Hochberg CH, Schneider JA, Dandona R, Lakshmi V, Kumar GA, Sudha T, et al. Population and dyadic-based seroincidence of herpes simplex virus-2 and syphilis in southern India. Sex Transm Infect. 2015;91(5):375-82.

272. Hocking JS, Guy R, Walker J, Tabrizi SN. Advances in sampling and screening for chlamydia. Future Microbiol. 2013;8(3):367-86.

273. Hoebe CJ, Rademaker CW, Brouwers EE, ter Waarbeek HL, van Bergen JE. Acceptability of self-taken vaginal swabs and first-catch urine samples for the diagnosis of urogenital Chlamydia trachomatis and Neisseria gonorrhoeae with an amplified DNA assay in young women attending a public health sexually transmitted disease clinic. Sex Transm Dis. 2006;33(8):491-5.

274. Hoehn EF, FitzGerald MR, Bhatt SR, Robinson VM, Lippe JE, Reed JL. Do Adolescents With Higher Knowledge of HIV Have Lower Sexual Risk Behaviors? Pediatr Emerg Care. 2016;32(12):846-50. 
275. Hogan AH, Howell-Jones RS, Pottinger E, Wallace LM, McNulty CA. "...they should be offering it": a qualitative study to investigate young peoples' attitudes towards chlamydia screening in GP surgeries. BMC Public Health. 2010;10:616.

276. Hogben M, Leichliter JS. Social determinants and sexually transmitted disease disparities. Sex Transm Dis. 2008;35(12 Suppl):S13-8.

277. Holmes KK, Levine R, Weaver M. Effectiveness of condoms in preventing sexually transmitted infections. Bull World Health Organ. 2004;82(6):454-61.

278. Hood JE, Friedman AL. Unveiling the hidden epidemic: a review of stigma associated with sexually transmissible infections. Sex Health. 2011;8(2):159-70.

279. Hook EW, 3rd, Richey CM, Leone P, Bolan G, Spalding C, Henry K, et al. Delayed presentation to clinics for sexually transmitted diseases by symptomatic patients. A potential contributor to continuing STD morbidity. Sex Transm Dis. 1997;24(8):443-8.

280. Hopkins S, Lyons F, Mulcahy F, Bergin C. The great pretender returns to Dublin, Ireland. Sex Transm Infect. 2001;77(5):316-8.

281. Horner PJ, Wills GS, Righarts A, Vieira S, Kounali D, Samuel D, et al. Chlamydia trachomatis Pgp3 Antibody Persists and Correlates with Self-Reported Infection and Behavioural Risks in a Blinded Cohort Study. PLoS One. 2016;11(3):e0151497.

282. Howell-Jones R, de Silva N, Akpan M, Oakeshott P, Carder C, Coupland L, et al. Prevalence of human papillomavirus (HPV) infections in sexually active adolescents and young women in England, prior to widespread HPV immunisation. Vaccine. 2012;30(26):3867-75.

283. Hsiao C, Hsieh S, Maranda EL, Lim V, Zullo J, Jimenez J. Syphilis, a disfiguring disease. JAMA Dermatology. 2016;152(4):404.

284. Hughes AJ, Saxton PJ. Thirty years of condom-based HIV prevention by gay men in New Zealand. N Z Med J. 2015;128(1426):19-30.

285. Hughes G, Catchpole M, Rogers PA, Brady AR, Kinghorn G, Mercey D, et al. Comparison of risk factors for four sexually transmitted infections: results from a study of attenders at three genitourinary medicine clinics in England. Sex Transm Infect. 2000;76(4):262-7.

286. Hughes JP, Baeten JM, Lingappa JR, Magaret AS, Wald A, de Bruyn G, et al. Determinants of per-coital-act HIV-1 infectivity among African HIV-1-serodiscordant couples. J Infect Dis. 2012;205(3):358-65.

287. Human Rights Campaign. Collecting Transgender-Inclusive Gender Data in Workplace and Other Surveys. Available at: http://www.hrc.org/resources/collecting-transgender-inclusivegender-data-in-workplace-and-other-surveys [Accessed 05/12/16]. 2016.

288. Humblet O, Paul C, Dickson N. Core group evolution over time: high-risk sexual behavior in a birth cohort between sexual debut and age 26. Sex Transm Dis. 2003;30(11):818-24. 
289. Hunziker S, Schlapfer M, Langewitz W, Kaufmann G, Nuesch R, Battegay E, et al. Open and hidden agendas of "asymptomatic" patients who request check-up exams. BMC Fam Pract. $2011 ; 12: 22$.

290. Hutter JN, Decker CF. Human papillomavirus infection. Dis Mon. 2016;62(8):294-300.

291. Hyde A, Drennan J, Howlett E, Brady D. Safer heterosex: perspectives from young men in Ireland. Sex Health. 2008;5(1):25-30.

292. Idahl A, Boman J, Kumlin U, Olofsson JI. Demonstration of Chlamydia trachomatis IgG antibodies in the male partner of the infertile couple is correlated with a reduced likelihood of achieving pregnancy. Hum Reprod. 2004;19(5):1121-6.

293. Iglesias-Gammara A, Restrepo JF, Valle R, Matteson EL. A Brief History of Stoll-BrodieFiessinger-Leroy Syndrome (Reiters Syndrome) and Reactive Arthritis with a Translation of Reiters Original 1916 Article into English. Curr Rheumatol Rev. 2005;1(1):71-9.

294. Ingabire CM, Kateera F, Hakizimana E, Rulisa A, Muvunyi C, Mens P, et al. Determinants of prompt and adequate care among presumed malaria cases in a community in eastern Rwanda: a cross sectional study. Malar J. 2016;15:227.

295. Ingham R. 'We didn't cover that at school': education against pleasure or education for pleasure? Sex Education. 2005;5(4):375-88.

296. Inman RD, Whittum-Hudson JA, Schumacher HR, Hudson AP. Chlamydia and associated arthritis. Curr Opin Rheumatol. 2000;12(4):254-62.

297. Ireland JA, Reid M, Powell R, Petrie KJ. The role of illness perceptions: psychological distress and treatment-seeking delay in patients with genital warts. Int J STD AIDS. 2005;16(10):667-70.

298. Irwin DE, Thomas JC, Spitters CE, Leone PA, Stratton JD, Martin DH, et al. Self-treatment patterns among clients attending sexually transmitted disease clinics and the effects of selftreatment on STD symptom duration. The Study Group. Sex Transm Dis. 1997;24(6):372-7.

299. Irwin DE, Thomas JC, Spitters CE, Leone PA, Stratton JD, Martin DH, et al. Self-reported sexual activity and condom use among symptomatic clients attending STD clinics. Sex Transm Dis. 1999;26(5):286-90.

300. Jaccard J, McDonald R, Wan CK, Guilamo-Ramos V, Dittus P, Quinlan S. Recalling sexual partners: the accuracy of self-reports. J Health Psychol. 2004;9(6):699-712.

301. Jackson LJ, Roberts TE. Conceptualising quality of life outcomes for women participating in testing for sexually transmitted infections: A systematic review and meta-synthesis of qualitative research. Soc Sci Med. 2015;143:162-70.

302. James SH, Kimberlin DW. Neonatal Herpes Simplex Virus Infection. Infect Dis Clin North Am. 2015;29(3):391-400.

303. Janeway CA, Travers P, Walport M, Shlomchik MJ. Immunobiology: The Immune System in Health and Disease. 5th edition. New York: Garland Science; 2001. 
304. Jaramillo-Rangel G, Gallegos-Avila G, Ramos-Gonzalez B, Alvarez-Cuevas S, MoralesGarcía AM, Javier Sanchez J, et al. The Role of Chlamydia trachomatis in Male Infertility. In: Mares M, editor. Chlamydia: InTech; 2012.

305. Javadnoori M, Roudsari RL, Hasanpour M, Hazavehei SM, Taghipour A. Female adolescents' experiences and perceptions regarding sexual health education in Iranian schools: A qualitative content analysis. Iran J Nurs Midwifery Res. 2012;17(7):539-46.

306. Javanbakht M, Gorbach P, Stirland A, Chien M, Kerndt P, Guerry S. Prevalence and correlates of rectal Chlamydia and gonorrhea among female clients at sexually transmitted disease clinics. Sex Transm Dis. 2012;39(12):917-22.

307. Jaworski BC, Carey MP. Development and psychometric evaluation of a self-administered questionnaire to measure knowledge of sexually transmitted diseases. AIDS Behav. 2007;11(4):557-74.

308. Jensen BL, Hoff G, Weismann K. A comparison of an enzyme immunoassay and cell culture for detection of Chlamydia trachomatis in genito-urinary specimens. Sex Transm Dis. 1988;15(2):123-6.

309. Johnson AM, Wadsworth J, Wellings K, Field J. Who goes to sexually transmitted disease clinics? Results from a national population survey. Genitourin Med. 1996;72(3):197-202.

310. Johnson HL, Ghanem KG, Zenilman JM, Erbelding EJ. Sexually transmitted infections and adverse pregnancy outcomes among women attending inner city public sexually transmitted diseases clinics. Sex Transm Dis. 2011;38(3):167-71.

311. Johnson MJ, Nemeth LS, Mueller M, Eliason MJ, Stuart GW. Qualitative Study of Cervical Cancer Screening Among Lesbian and Bisexual Women and Transgender Men. Cancer Nurs. 2016;39(6):455-63.

312. Jolly AM, Muth SQ, Wylie JL, Potterat JJ. Sexual networks and sexually transmitted infections: a tale of two cities. J Urban Health. 2001;78(3):433-45.

313. Jorgensen MJ, Maindal HT, Larsen MB, Christensen KS, Olesen F, Andersen B. Chlamydia trachomatis infection in young adults - association with concurrent partnerships and short gap length between partners. Infect Dis (Lond). 2015;47(12):838-45.

314. Juckett G, Hartman-Adams H. Human papillomavirus: clinical manifestations and prevention. Am Fam Physician. 2010;82(10):1209-13.

315. Jutel A. Beyond the Sociology of Diagnosis. Sociology Compass. 2015;9(9):841-52.

316. Jutel A, Banister E. "I was pretty sure I had the 'flu": qualitative description of confirmedinfluenza symptoms. Soc Sci Med. 2013;99:49-55.

317. Kamb ML, Rhodes F, Hoxworth T, Rogers J, Lentz A, Kent C, et al. What about money? Effect of small monetary incentives on enrollment, retention, and motivation to change behaviour in an HIV/STD prevention counselling intervention. The Project RESPECT Study Group. Sex Transm Infect. 1998;74(4):253-5. 
318. Kang M, Rochford A, Skinner SR, Mindel A, Webb M, Peat J, et al. Sexual behaviour, sexually transmitted infections and attitudes to chlamydia testing among a unique national sample of young Australians: baseline data from a randomised controlled trial. BMC Public Health. 2014;14:12.

319. Karam E, Kypri K, Salamoun M. Alcohol use among college students: an international perspective. Curr Opin Psychiatry. 2007;20(3):213-21.

320. Keat A, Thomas B, Dixey J, Osborn M, Sonnex C, Taylor-Robinson D. Chlamydia trachomatis and reactive arthritis: the missing link. Lancet. 1987;1(8524):72-4.

321. Keat AC, Maini RN, Nkwazi GC, Pegrum GD, Ridgway GL, Scott JT. Role of Chlamydia trachomatis and HLA-B27 in sexually acquired reactive arthritis. BMJ. 1978;1(6113):605-7.

322. Keck C, Gerber-Schafer C, Clad A, Wilhelm C, Breckwoldt M. Seminal tract infections: impact on male fertility and treatment options. Hum Reprod Update. 1998;4(6):891-903.

323. Keeling MJ, Rohani P. Modeling infectious diseases in humans and animals: Princeton University Press; 2008.

324. Keithlin J, Sargeant J, Thomas MK, Fazil A. Systematic review and meta-analysis of the proportion of Campylobacter cases that develop chronic sequelae. BMC Public Health. $2014 ; 14: 1203$

325. Keithlin J, Sargeant JM, Thomas MK, Fazil A. Systematic review and meta-analysis of the proportion of non-typhoidal Salmonella cases that develop chronic sequelae. Epidemiol Infect. 2015;143(7):1333-51.

326. Kellock DJ, Piercy H, Rogstad KE. Knowledge of Chlamydia trachomatis infection in genitourinary medicine clinic attenders. Sex Transm Infect. 1999;75(1):36-40.

327. Kelly MP, Barker M. Why is changing health-related behaviour so difficult? Public Health. 2016;136:109-16.

328. Kerry SR, Nightingale CM, Hay P, Oakeshott P. Which sexually active female students get themselves tested for Chlamydia trachomatis? A cohort study. Int J STD AIDS. 2015;27(7):586-90.

329. Khan A, Fortenberry JD, Temkit MH, Tu W, Orr DP, Batteiger BE. Gender differences in sexual behaviours in response to genitourinary symptoms. Sex Transm Infect. 2005;81(3):262-6.

330. Khan MR, Golin CE, Friedman SR, Scheidell JD, Adimora AA, Judon-Monk S, et al. STI/HIV Sexual Risk Behavior and Prevalent STI Among Incarcerated African American Men in Committed Partnerships: The Significance of Poverty, Mood Disorders, and Substance Use. AIDS Behav. 2015;19(8):1478-90.

331. Kilgour R, Maskill C, Lungley S. Public perceptions of AIDS: knowledge, attitudes and behaviour. Wellington: Health Research Services, Department of Health, 1990. 
332. Kirby DB, Laris BA, Rolleri LA. Sex and HIV education programs: their impact on sexual behaviors of young people throughout the world. J Adolesc Health. 2007;40(3):206-17.

333. The Kirby Institute. HIV, viral hepatitis and sexually transmissible infections in Australia. Annual Surveillance Report. UNSW Australia, Sydney: 2015.

334. The Kirby Institute. HIV, viral hepatitis and sexually transmissible infections in Australia. Annual Surveillance Report. UNSW Australia, Sydney: 2016.

335. Kissinger P. Trichomonas vaginalis: a review of epidemiologic, clinical and treatment issues. BMC Infect Dis. 2015;15:307.

336. Kitzinger J. Qualitative research. Introducing focus groups. BMJ. 1995;311(7000):299-302.

337. Klovstad H, Grjibovski A, Aavitsland P. Population based study of genital Chlamydia trachomatis prevalence and associated factors in Norway: a cross sectional study. BMC Infect Dis. 2012;12:150.

338. Kong FY, Hocking JS, Link CK, Chen MY, Hellard ME. Sex and sport: chlamydia screening in rural sporting clubs. BMC Infect Dis. 2009;9:73.

339. Kong FY, Hocking JS, Link CK, Chen MY, Hellard ME. Sex and sport: sexual risk behaviour in young people in rural and regional Victoria. Sex Health. 2010;7(2):205-11.

340. Koren M, Decker CF. Genital herpes. Dis Mon. 2016;62(8):287-93.

341. Krantz I, Lowhagen GB, Ahlberg BM, Nilstun T. Ethics of screening for asymptomatic herpes virus type 2 infection. BMJ. 2004;329(7466):618-21.

342. Kraut-Becher JR, Aral SO. Gap length: an important factor in sexually transmitted disease transmission. Sex Transm Dis. 2003;30(3):221-5.

343. Kuipers JG, Kohler L, Zeidler H. Reactive or infectious arthritis. Ann Rheum Dis. 1999;58(11):661-4.

344. Kvien TK, Glennas A, Melby K, Granfors K, Andrup O, Karstensen B, et al. Reactive arthritis: incidence, triggering agents and clinical presentation. J Rheumatol. 1994;21(1):11522.

345. Kypri K, Langley JD, McGee R, Saunders JB, Williams S. High prevalence, persistent hazardous drinking among New Zealand tertiary students. Alcohol Alcohol. 2002;37(5):45764.

346. Lachowsky NJ, Saxton PJ, Dickson NP, Hughes AJ, Summerlee AJ, Dewey CE. Factors associated with recent HIV testing among younger gay and bisexual men in New Zealand, 2006-2011. BMC Public Health. 2014;14:294.

347. Lancet. Time to take sexually transmitted infections seriously. Lancet Infect Dis. 2016;16(9):981. 
348. Langenberg AG, Corey L, Ashley RL, Leong WP, Straus SE. A prospective study of new infections with herpes simplex virus type 1 and type 2. Chiron HSV Vaccine Study Group. $N$ Engl J Med. 1999;341(19):1432-8.

349. Langille DB, Flowerdew G, Aquino-Russell C, Strang R, Proudfoot K, Forward K. Gender differences in knowledge about chlamydia among rural high school students in Nova Scotia, Canada. Sex Health. 2009;6(1):11-4.

350. Larsen B, Monif GR. Understanding the bacterial flora of the female genital tract. Clin Infect Dis. 2001;32(4):e69-77.

351. Latreille S, Collyer A, Temple-Smith M. Finding a segue into sex: young men's views on discussing sexual health with a GP. Aust Fam Physician. 2014;43(4):217-21.

352. Laumann EO, Youm Y. Racial/ethnic group differences in the prevalence of sexually transmitted diseases in the United States: a network explanation. Sex Transm Dis. 1999;26(5):250-61.

353. Lavikainen HM, Lintonen T, Kosunen E. Sexual behavior and drinking style among teenagers: a population-based study in Finland. Health Promot Int. 2009;24(2):108-19.

354. Lawless S. Sustaining chlamydia screening is difficult. J Prim Health Care. 2010;2(4):347.

355. Lawson E, Walker-Bone K. The changing spectrum of rheumatic disease in HIV infection. $\mathrm{Br}$ Med Bull. 2012;103(1):203-21.

356. Lawton B, Bromhead C. Nucleic acid amplification tests of self-taken vulvovaginal swabs are more sensitive than clinician taken endocervical culture for gonorrhoea. Evid Based Med. 2013;18(5):e46.

357. Lawton B, Rose S, Bromhead C, Brown S, MacDonald J, Shepherd J. Rates of Chlamydia trachomatis testing and chlamydial infection in pregnant women. $N Z$ Med $J$. 2004;117(1194):U889.

358. Lawton BA, Rose SB, Elley CR, Bromhead C, MacDonald EJ, Baker MG. Increasing the uptake of opportunistic chlamydia screening: a pilot study in general practice. J Prim Health Care. 2010;2(3):199-207.

359. Lazarus JV, Sihvonen-Riemenschneider H, Laukamm-Josten U, Wong F, Liljestrand J. Systematic review of interventions to prevent the spread of sexually transmitted infections, including HIV, among young people in Europe. Croat Med J. 2010;51(1):74-84.

360. Lazenby GB. Opportunistic infections in women with HIV AIDS. Clin Obstet Gynecol. 2012;55(4):927-37.

361. Lee H, Unemo M, Kim HJ, Seo Y, Lee K, Chong Y. Emergence of decreased susceptibility and resistance to extended-spectrum cephalosporins in Neisseria gonorrhoeae in Korea. $J$ Antimicrob Chemother. 2015;70(9):2536-42.

362. Lee V, Tobin JM, Foley E. Relationship of cervical ectopy to chlamydia infection in young women. J Fam Plann Reprod Health Care. 2006;32(2):104-6. 
363. Leenaars P, R. Rombouts, B., Kok, G. Seeking medical care for a Sexually Transmitted Disease: Determinants of delay-behavior. Psychol Health. 1993;8(1):17-32.

364. LeFevre ML. U.S. Preventive Services Task Force (USPSTF): screening for chlamydia and gonorrhea. Ann Intern Med. 2014;161:902-10.

365. Levin EM, Koopman JS, Aral SO, Holmes KK, Foxman B. Characteristics of men who have sex with men and women and women who have sex with women and men: results from the 2003 Seattle sex survey. Sex Transm Dis. 2009;36(9):541-6.

366. Lewis JE, Miguez-Burban, M., Malow, R. M. HIV Risk Behavior among College Students in the United States. College Student Journal. 2009;42:475-91.

367. Lewis P, Hensel M, Emerman M. Human immunodeficiency virus infection of cells arrested in the cell cycle. EMBO J. 1992;11(8):3053-8.

368. Li D, Yang X, Zhang Z, Wang Z, Qi X, Ruan Y, et al. Incidence of Co-Infections of HIV, Herpes Simplex Virus Type 2 and Syphilis in a Large Cohort of Men Who Have Sex with Men in Beijing, China. PLoS One. 2016;11(1):e0147422.

369. Lichtenstein B. Stigma as a barrier to treatment of sexually transmitted infection in the American deep south: issues of race, gender and poverty. Soc Sci Med. 2003;57(12):2435-45.

370. Lim MS, Hocking JS, Aitken CK, Fairley CK, Jordan L, Lewis JA, et al. Impact of text and email messaging on the sexual health of young people: a randomised controlled trial. $J$ Epidemiol Community Health. 2012;66(1):69-74.

371. Lippman SA, Jones HE, Luppi CG, Pinho AA, Veras MA, van de Wijgert JH. Home-based self-sampling and self-testing for sexually transmitted infections: acceptable and feasible alternatives to provider-based screening in low-income women in Sao Paulo, Brazil. Sex Transm Dis. 2007;34(7):421-8.

372. Litras A, Latreille S, Temple-Smith M. Dr Google, porn and friend-of-a-friend: where are young men really getting their sexual health information? Sex Health. 2015;12(6):488-94.

373. Little RJ, D'Agostino R, Cohen ML, Dickersin K, Emerson SS, Farrar JT, et al. The prevention and treatment of missing data in clinical trials. N Engl J Med. 2012;367(14):135560.

374. Liu B, Roberts CL, Clarke M, Jorm L, Hunt J, Ward J. Chlamydia and gonorrhoea infections and the risk of adverse obstetric outcomes: a retrospective cohort study. Sex Transm Infect. 2013;89(8):672-8.

375. Liu H, Detels R, Li X, Ma E, Yin Y. Stigma, delayed treatment, and spousal notification among male patients with sexually transmitted disease in China. Sex Transm Dis. 2002;29(6):335-43.

376. Llewellyn C, Pollard A, Miners A, Richardson D, Fisher M, Cairns J, et al. Understanding patient choices for attending sexually transmitted infection testing services: a qualitative study. Sex Transm Infect. 2012;88(7):504-9. 
377. Llewellyn C, Pollard A, Smith H, Fisher M. Are home sampling kits for sexually transmitted infections acceptable among men who have sex with men? J Health Serv Res Policy. 2009;14(1):35-43.

378. Lloyd GE. Hippocratic writings (translated by J. Chadwick and W. N. Mann). New York, New York: Pelican Books; 1978.

379. Lo M, Reid M, Brokenshire M. Epidemiological features of women with trichomoniasis in Auckland sexual health clinics: 1998-99. N Z Med J. 2002;115(1159):U119.

380. Lopez-Zeno JA, Keith LG, Berger GS. The Fitz-Hugh-Curtis syndrome revisited. Changing perspectives after half a century. J Reprod Med. 1985;30(8):567-82.

381. Lorimer K, Hart GJ. Knowledge of Chlamydia trachomatis among men and women approached to participate in community-based screening, Scotland, UK. BMC Public Health. 2010;10:794.

382. Low N. Screening programmes for chlamydial infection: when will we ever learn? BMJ. 2007;334(7596):725-8.

383. Low N, Egger M. What should we do about screening for genital chlamydia? Int J Epidemiol. 2002;31(5):891-3.

384. Low N, Egger M, Sterne JA, Harbord RM, Ibrahim F, Lindblom B, et al. Incidence of severe reproductive tract complications associated with diagnosed genital chlamydial infection: the Uppsala Women's Cohort Study. Sex Transm Infect. 2006;82(3):212-8.

385. Low N, Redmond S, Uuskula A, van Bergen J, Ward H, Andersen B, et al. Screening for genital chlamydia infection. Cochrane Database Syst Rev. 2016;9:Cd010866.

386. Ludlam AH, Saxton PJ, Dickson NP, Hughes AJ. General practitioner awareness of sexual orientation among a community and internet sample of gay and bisexual men in New Zealand. J Prim Health Care. 2015;7(3):204-12.

387. Lungley S. Intravenous drug use in New Zealand: A baseline study of intravenous drug users and the risk of AIDS. Discussion Paper No. 2. Available at http://www.moh.govt.nz/notebook/nbbooks.nsf/0/5f6817ca5067bcee4c2565d7001874fa/\$FIL E/Intravenous.pdf [Accessed 04/12/16]. Wellington: Department of Health, 1988.

388. Lyamuya EF, Aboud S, Urassa WK, Sufi J, Mbwana J, Ndugulile F, et al. Evaluation of simple rapid HIV assays and development of national rapid HIV test algorithms in Dar es Salaam, Tanzania. BMC Infect Dis. 2009;9:19.

389. Lycke E, Lowhagen GB, Hallhagen G, Johannisson G, Ramstedt K. The risk of transmission of genital Chlamydia trachomatis infection is less than that of genital Neisseria gonorrhoeae infection. Sex Transm Dis. 1980;7(1):6-10.

390. Macdonald G. The analysis of equilibrium in malaria. Trop Dis Bull. 1952;49(9):813-29.

391. Macdowall W, Jones KG, Tanton C, Clifton S, Copas AJ, Mercer CH, et al. Associations between source of information about sex and sexual health outcomes in Britain: findings from 
the third National Survey of Sexual Attitudes and Lifestyles (Natsal-3). BMJ Open. 2015;5(3):e007837.

392. Mackern-Oberti JP, Motrich RD, Breser ML, Sanchez LR, Cuffini C, Rivero VE. Chlamydia trachomatis infection of the male genital tract: an update. J Reprod Immunol. 2013;100(1):3753.

393. MacKian S. A review of health seeking behaviour: problems and prospects. Internal concept paper. London: Health Systems Development Programme, London School of Hygiene and Tropical Medicine, 2001.

394. Macleod J, Salisbury C, Low N, McCarthy A, Sterne JA, Holloway A, et al. Coverage and uptake of systematic postal screening for genital Chlamydia trachomatis and prevalence of infection in the United Kingdom general population: cross sectional study. BMJ.

2005;330(7497):940.

395. Maddox J. Sexual behaviour unsurveyed. Nature. 1989;341(6239):181.

396. Major B, O'Brien LT. The social psychology of stigma. Annu Rev Psychol. 2005;56:393-421.

397. Malacova E, Butler T, Richters J, Yap L, Grant L, Richards A, et al. Knowledge of sexually transmissible infections: a comparison of prisoners and the general population. Int J STD AIDS. 2011;22(7):381-6.

398. Malek AM, Chang CC, Clark DB, Cook RL. Delay in Seeking Care for Sexually Transmitted Diseases in Young Men and Women Attending a Public STD Clinic. Open AIDS J. 2013;7:713.

399. Malhotra M, Sood S, Mukherjee A, Muralidhar S, Bala M. Genital Chlamydia trachomatis: an update. Indian J Med Res. 2013;138(3):303-16.

400. Malta M, Bastos FI, Strathdee SA, Cunnigham SD, Pilotto JH, Kerrigan D. Knowledge, perceived stigma, and care-seeking experiences for sexually transmitted infections: a qualitative study from the perspective of public clinic attendees in Rio de Janeiro, Brazil. BMC Public Health. 2007;7:18.

401. Manavi K. A review on infection with human immunodeficiency virus. Best Pract Res Clin Obstet Gynaecol. 2006;20(6):923-40.

402. Mansuy JM, Dutertre M, Mengelle C, Fourcade C, Marchou B, Delobel P, et al. Zika virus: high infectious viral load in semen, a new sexually transmitted pathogen? Lancet Infect Dis. 2016;16(4):405.

403. Mapp F, Hickson F, Mercer CH, Wellings K. How social representations of sexually transmitted infections influence experiences of genito-urinary symptoms and care-seeking in Britain: mixed methods study protocol. BMC Public Health. 2016;16:548.

404. Mardh PA. Influence of infection with Chlamydia trachomatis on pregnancy outcome, infant health and life-long sequelae in infected offspring. Best Pract Res Clin Obstet Gynaecol. 2002;16(6):847-64. 
405. Marlow LA, Zimet GD, McCaffery KJ, Ostini R, Waller J. Knowledge of human papillomavirus (HPV) and HPV vaccination: an international comparison. Vaccine. 2013;31(5):763-9.

406. Martinez JL, Baquero F. Interactions among strategies associated with bacterial infection: pathogenicity, epidemicity, and antibiotic resistance. Clin Microbiol Rev. 2002;15(4):647-79.

407. Martyniuk U, Briken P, Sehner S, Richter-Appelt H, Dekker A. Pornography Use and Sexual Behavior Among Polish and German University Students. J Sex Marital Ther. 2016;42(6):494-514.

408. Mason L. Knowledge of sexually transmitted infections and sources of information amongst men. J R Soc Promot Health. 2005;125(6):266-71.

409. Masson L, Passmore JA, Liebenberg LJ, Werner L, Baxter C, Arnold KB, et al. Genital inflammation and the risk of HIV acquisition in women. Clin Infect Dis. 2015;61(2):260-9.

410. Masuda N, Holme P. Predicting and controlling infectious disease epidemics using temporal networks. F1000 Prime Rep. 2013;5:6.

411. Maurice WL. Sexual Medicine in Primary Care. St Louis, U.S: Mosby; 1999.

412. Mayer KH. Sexually transmitted diseases in men who have sex with men. Clin Infect Dis. 2011;53 Suppl 3:S79-83.

413. Mayer KH, Bradford JB, Makadon HJ, Stall R, Goldhammer H, Landers S. Sexual and gender minority health: what we know and what needs to be done. Am J Public Health. 2008;98(6):989-95.

414. Mazzoli S, Cai T, Addonisio P, Bechi A, Mondaini N, Bartoletti R. Chlamydia trachomatis infection is related to poor semen quality in young prostatitis patients. Eur Urol. 2010;57(4):708-14.

415. McCabe MP, Cummins RA, Deeks AA. Construction and psychometric properties of sexuality scales: sex knowledge, experience, and needs scales for people with intellectual disabilities (SexKen-ID), people with physical disabilities (SexKen-PD), and the general population (SexKen-GP). Res Dev Disabil. 1999;20(4):241-54.

416. McCall H, Adams N, Mason D, Willis J. What is chemsex and why does it matter? BMJ. 2015;351:h5790.

417. McCarthy M. Zika virus was transmitted by sexual contact in Texas, health officials report. BMJ. 2016;352:i720.

418. McClean H, Sutherland J, Searle S, Howarth P. An exploratory study of information-giving used to promote chlamydial test-seeking by students at a college family planning clinic. $\mathrm{Br} J$ Fam Plann. 2000;26(4):209-12.

419. McClean HL, Reid M. Use of GUM services and information and views held by first time service users in a large UK city: implications for information provision. Int J STD AIDS. 1997;8(3):154-8. 
420. Meader N, King K, Moe-Byrne T, Wright K, Graham H, Petticrew M, et al. A systematic review on the clustering and co-occurrence of multiple risk behaviours. BMC Public Health. 2016;16:657.

421. Meites E, Gaydos CA, Hobbs MM, Kissinger P, Nyirjesy P, Schwebke JR, et al. A Review of Evidence-Based Care of Symptomatic Trichomoniasis and Asymptomatic Trichomonas vaginalis Infections. Clin Infect Dis. 2015;61 Suppl 8:S837-48.

422. Melendez-Torres GJ, Bourne A. Illicit drug use and its association with sexual risk behaviour among MSM: more questions than answers? Curr Opin Infect Dis. 2016;29(1):58-63.

423. Melville J, Sniffen S, Crosby R, Salazar L, Whittington W, Dithmer-Schreck D, et al. Psychosocial impact of serological diagnosis of herpes simplex virus type 2: a qualitative assessment. Sex Transm Infect. 2003;79(4):280-5.

424. Meneses-Gaya C, Zuardi AW, Loureiro SR, Hallak JE, Trzesniak C, de Azevedo Marques $\mathrm{JM}$, et al. Is the full version of the AUDIT really necessary? Study of the validity and internal construct of its abbreviated versions. Alcohol Clin Exp Res. 2010;34(8):1417-24.

425. Mercer CH, Aicken CR, Cassell JA, Hartnell V, Davies L, Ryan J, et al. Not so different after all? Comparing patients attending general practice-based locally enhanced services for sexual health with patients attending genitourinary medicine. Int J STD AIDS. 2013a;24(2):106-11.

426. Mercer CH, Aicken CR, Estcourt CS, Keane F, Brook G, Rait G, et al. Building the bypassimplications of improved access to sexual healthcare: evidence from surveys of patients attending contrasting genitourinary medicine clinics across England in 2004/2005 and 2009. Sex Transm Infect. 2012;88(1):9-15.

427. Mercer CH, Aicken CR, Tanton C, Estcourt CS, Brook MG, Keane F, et al. Serial monogamy and biologic concurrency: measurement of the gaps between sexual partners to inform targeted strategies. Am J Epidemiol. 2013b;178(2):249-59.

428. Mercer CH, Hart GJ, Johnson AM, Cassell JA. Behaviourally bisexual men as a bridge population for HIV and sexually transmitted infections? Evidence from a national probability survey. Int J STD AIDS. 2009;20(2):87-94.

429. Mercer CH, Sutcliffe L, Johnson AM, White PJ, Brook G, Ross JD, et al. How much do delayed healthcare seeking, delayed care provision, and diversion from primary care contribute to the transmission of STIs? Sex Transm Infect. 2007;83(5):400-5.

430. Mercer CH, Tanton C, Prah P, Erens B, Sonnenberg P, Clifton S, et al. Changes in sexual attitudes and lifestyles in Britain through the life course and over time: findings from the National Surveys of Sexual Attitudes and Lifestyles (Natsal). Lancet. 2013c;382(9907):178194.

431. Merritt TD, Durrheim DN, Hope K, Byron P. General practice intervention to increase opportunistic screening for chlamydia. Sex Health. 2007;4(4):249-51.

432. Meyer-Weitz A, Reddy P, Van den Borne HW, Kok G, Pietersen J. Health care seeking behaviour of patients with sexually transmitted diseases: determinants of delay behaviour. Patient Educ Couns. 2000;41(3):263-74. 
433. Miller WC, Ford CA, Morris M, Handcock MS, Schmitz JL, Hobbs MM, et al. Prevalence of chlamydial and gonococcal infections among young adults in the United States. JAMA. 2004;291(18):2229-36.

434. Mills N, Daker-White G, Graham A, Campbell R. Population screening for Chlamydia trachomatis infection in the UK: a qualitative study of the experiences of those screened. Fam Pract. 2006;23(5):550-7.

435. Mimiaga MJ, Goldhammer H, Belanoff C, Tetu AM, Mayer KH. Men who have sex with men: perceptions about sexual risk, HIV and sexually transmitted disease testing, and provider communication. Sex Transm Dis. 2007;34(2):113-9.

436. Ministry of Education. Sexuality Education: A guide for principals, boards of trustees, and teachers. Wellington, New Zealand: 2015.

437. Ministry of Health. Sexual and Reproductive Health: A resource book for New Zealand health care organisations. Wellington, New Zealand: 2003.

438. Mlisana K, Naicker N, Werner L, Roberts L, van Loggerenberg F, Baxter C, et al. Symptomatic vaginal discharge is a poor predictor of sexually transmitted infections and genital tract inflammation in high-risk women in South Africa. J Infect Dis. 2012;206(1):614.

439. Mmbaga EJ, Leyna GH, Mnyika KS, Klepp KI. Sexually transmitted infections knowledge and its impact in the practice of risky sexual behaviours and HIV serostatus: results from rural Kilimanjaro, Tanzania. Sex Transm Infect. 2008;84(3):224-6.

440. Moher D, Liberati A, Tetzlaff J, Altman DG. Preferred Reporting Items for Systematic Reviews and Meta-Analyses: The PRISMA Statement. J Clin Epidemiol. 2009;62(10):100612.

441. Moi H, Blee K, Horner PJ. Management of non-gonococcal urethritis. BMC Infect Dis. 2015;15:294.

442. Molano M, Meijer CJ, Weiderpass E, Arslan A, Posso H, Franceschi S, et al. The natural course of Chlamydia trachomatis infection in asymptomatic Colombian women: a 5-year follow-up study. J Infect Dis. 2005;191(6):907-16.

443. Moreira ED, Jr., Brock G, Glasser DB, Nicolosi A, Laumann EO, Paik A, et al. Help-seeking behaviour for sexual problems: the global study of sexual attitudes and behaviors. Int J Clin Pract. 2005;59(1):6-16.

444. Morgan J. Testing and detection trends of Chlamydia trachomatis and Neisseria gonorrhoeae in Waikato, New Zealand: 1998-2006. N Z Med J. 2008;121(1278):41-9.

445. Morgan J, Bell A. The highs and lows of opportunistic Chlamydia testing: uptake and detection in Waikato, New Zealand. Sex Transm Infect. 2009;85(6):452-4.

446. Morgan J, Donnell A, Bell A. Is everyone treated equally? Management of genital Chlamydia trachomatis infection in New Zealand. Int J STD AIDS. 2010;21(8):595-600. 
447. Morgan J, Donnell A, Bell A. Does a clinical guideline change chlamydia testing? Report from the Waikato Chlamydia Project. J Prim Health Care. 2012;4(1):45-51.

448. Morgan J, Haar J. Who goes to a sexual health clinic? Gender differences in service utilisation. N Z Med J. 2008;121(1287):44-9.

449. Morgan J, Haar J. General practice funding to improve provision of adolescent primary sexual health care in New Zealand: results from an observational intervention. Sex Health. 2009;6(3):203-7.

450. Morgan JM. Epidemiology, screening and management of Chlamydia trachomatis infection in New Zealand (Doctoral thesis): The University of Auckland; 2013.

451. Morgan MK, Decker CF. Gonorrhea. Dis Mon. 2016;62(8):260-8.

452. Morre SA, van den Brule AJ, Rozendaal L, Boeke AJ, Voorhorst FJ, de Blok S, et al. The natural course of asymptomatic Chlamydia trachomatis infections: $45 \%$ clearance and no development of clinical PID after one-year follow-up. Int J STD AIDS. 2002;13 Suppl 2:12-8.

453. Morris D, Inman RD. Reactive arthritis: developments and challenges in diagnosis and treatment. Curr Rheumatol Rep. 2012;14(5):390-4.

454. Morris JL, Lippman SA, Philip S, Bernstein K, Neilands TB, Lightfoot M. Sexually transmitted infection related stigma and shame among African American male youth: implications for testing practices, partner notification, and treatment. AIDS Patient Care STDS. 2014;28(9):499-506.

455. Morris JL, Rushwan H. Adolescent sexual and reproductive health: The global challenges. Int J Gynaecol Obstet. 2015;131 Suppl 1:S40-2.

456. Morris M, Kretzschmar M. Concurrent partnerships and transmission dynamics in networks. Social Networks. 1995;17(3):299-318.

457. Moses S, Ngugi EN, Bradley JE, Njeru EK, Eldridge G, Muia E, et al. Health care-seeking behavior related to the transmission of sexually transmitted diseases in Kenya. Am J Public Health. 1994;84(12):1947-51.

458. Mulholland E, Van Wersch A. Stigma, sexually transmitted infections and attendance at the GUM Clinic: an exploratory study with implications for the theory of planned behaviour. $J$ Health Psychol. 2007;12(1):17-31.

459. Musso D, Gubler DJ. Zika Virus. Clin Microbiol Rev. 2016;29(3):487-524.

460. Mustanski B, Starks T, Newcomb ME. Methods for the design and analysis of relationship and partner effects on sexual health. Arch Sex Behav. 2014;43(1):21-33.

461. Myers JL. Why do young women get tested for sexually transmitted infections? Evidence from the National Longitudinal Study of Adolescent Health. J Womens Health (Larchmt). 2011;20(8):1225-31. 
462. Nack A. Damaged goods: women managing the stigma of STDs. Deviant Behavior. 2000;21(2):95-121.

463. Nahmias AJ, Dowdle WR. Antigenic and biologic differences in herpesvirus hominis. Prog Med Virol. 1968;10:110-59.

464. Naidoo S, Taylor M. HIV health literacy, sexual behaviour and self-reports of having tested for HIV among students. Afr J AIDS Res. 2015;14(2):107-15.

465. Nardini P, Compri M, Marangoni A, D'Antuono A, Bellavista S, Calvanese C, et al. Acute Fitz-Hugh-Curtis syndrome in a man due to gonococcal infection. J Emerg Med. 2015;48(3):e59-62.

466. Nassif X, Pujol C, Morand P, Eugene E. Interactions of pathogenic Neisseria with host cells. Is it possible to assemble the puzzle? Mol Microbiol. 1999;32(6):1124-32.

467. National Survey of Adolescents and Young Adults: Sexual Health Knowledge, Attitudes and Experiences. CA, USA: The Henry J. Kaiser Family Foundation, 2003.

468. Needham HE, Wiemann CM, Tortolero SR, Chacko MR. Relationship between health literacy, reading comprehension, and risk for sexually transmitted infections in young women. J Adolesc Health. 2010;46(5):506-8.

469. Nelson SJ, Manhart LE, Gorbach PM, Martin DH, Stoner BP, Aral SO, et al. Measuring sex partner concurrency: it's what's missing that counts. Sex Transm Dis. 2007;34(10):801-7.

470. Nesoff ED, Dunkle K, Lang D. The Impact of Condom Use Negotiation Self-Efficacy and Partnership Patterns on Consistent Condom Use Among College-Educated Women. Health Educ Behav. 2016;43(1):61-7.

471. Newman L, Rowley J, Vander Hoorn S, Wijesooriya NS, Unemo M, Low N, et al. Global Estimates of the Prevalence and Incidence of Four Curable Sexually Transmitted Infections in 2012 Based on Systematic Review and Global Reporting. PLoS One. 2015;10(12):e0143304.

472. Newton DC, McCabe MP. A theoretical discussion of the impact of stigma on psychological adjustment to having a sexually transmissible infection. Sex Health. 2005;2(2):63-9.

473. Nicastri E, Castilletti C, Liuzzi G, Iannetta M, Capobianchi MR, Ippolito G. Persistent detection of Zika virus RNA in semen for six months after symptom onset in a traveller returning from Haiti to Italy, February 2016. Euro Surveill. 2016;21(32).

474. Norbu K, Mukhia S, Tshokey. Assessment of knowledge on sexually transmitted infections and sexual risk behaviour in two rural districts of Bhutan. BMC Public Health. 2013;13(1):1142.

475. Nordvik MK, Liljeros F. Number of sexual encounters involving intercourse and the transmission of sexually transmitted infections. Sex Transm Dis. 2006;33(6):342-9. 
476. Nyatsanza F, Trivedy A, Brook G. The effect of introducing routine self-taken extra-genital swabs in a genitourinary medicine clinic cohort: a before and after study. Int J STD AIDS. 2016;27(14):1330-3.

477. NZSHS. Chlamydia Management Guidelines. Wellington, New Zealand: Ministry of Health, 2008.

478. NZSHS. Chlamydia Management Guidelines. Wellington, New Zealand: Ministry of Health, 2015a.

479. NZSHS. Guidelines for the Management of Genital, Anal and Throat HPV Infection in New Zealand. 8th Edition. Sexually Transmitted Infection Education Foundation. New Zealand Sexual Health Society, 2015b.

480. NZSHS. New Zealand Guideline for the Management of Gonorrhoea, 2014, and Response to the Threat of Antimicrobial Resistance. Gonorrhoea Guideline Writing Group on behalf of the New Zealand Sexual Health Society, 2014.

481. NZSHS. Sexual Health Check Management Guidelines. Best Practice Guide. New Zealand Sexual Health Society, 2015c.

482. NZSHS. Sexually Transmitted Infections. Summary of Guidelines. New Zealand Sexual Health Society, 2015 d.

483. O'Connor CC, Ali H, Guy RJ, Templeton DJ, Fairley CK, Chen MY, et al. High chlamydia positivity rates in Indigenous people attending Australian sexual health services. Med J Aust. 2014;200(10):595-8.

484. Oakeshott P, Kerry S, Aghaizu A, Atherton H, Hay S, Taylor-Robinson D, et al. Randomised controlled trial of screening for Chlamydia trachomatis to prevent pelvic inflammatory disease: the POPI (prevention of pelvic infection) trial. BMJ. 2010;340:c1642.

485. Odesanmi TY, Wasti SP, Odesanmi OS, Adegbola O, Oguntuase OO, Mahmood S. Comparative effectiveness and acceptability of home-based and clinic-based sampling methods for sexually transmissible infections screening in females aged 14-50 years: a systematic review and meta-analysis. Sex Health. 2013;10(6):559-69.

486. Office for National Statistics. Drug misuse: Findings from the 2013/14 crime survey for England and Wales. London: Office for National Statistics, 2014.

487. Oliver de Visser R, O'Neill N. Identifying and understanding barriers to sexually transmissible infection testing among young people. Sex Health. 2013;10(6):553-8.

488. Oriel JD. The Scars of Venus: A History of Venereology. London, United Kingdom: Springer-Verlag; 1994.

489. Ottesen B, Pedersen B, Nielsen J, Dalgaard D, Wagner G, Fahrenkrug J. Vasoactive intestinal polypeptide (VIP) provokes vaginal lubrication in normal women. Peptides. 1987;8(5):797800 . 
490. Owen DH, Katz DF. A review of the physical and chemical properties of human semen and the formulation of a semen simulant. J Androl. 2005;26(4):459-69.

491. Owlia MB, Eley AR. Is the role of Chlamydia trachomatis underestimated in patients with suspected reactive arthritis? Int J Rheum Dis. 2010;13(1):27-38.

492. Ozgul A, Dede I, Taskaynatan MA, Aydogan H, Kalyon TA. Clinical presentations of chlamydial and non-chlamydial reactive arthritis. Rheumatol Int. 2006;26(10):879-85.

493. Pacheco-Tena C, Burgos-Vargas R, Vazquez-Mellado J, Cazarin J, Perez-Diaz JA. A proposal for the classification of patients for clinical and experimental studies on reactive arthritis. J Rheumatol. 1999;26(6):1338-46.

494. Panaretto KS, Lee HM, Mitchell MR, Larkins SL, Manessis V, Buettner PG, et al. Prevalence of sexually transmitted infections in pregnant urban Aboriginal and Torres Strait Islander women in northern Australia. Aust N Z J Obstet Gynaecol. 2006;46(3):217-24.

495. Pararas MV, Skevaki CL, Kafetzis DA. Preterm birth due to maternal infection: Causative pathogens and modes of prevention. Eur J Clin Microbiol Infect Dis. 2006;25(9):562-9.

496. Parks KS, Dixon PB, Richey CM, Hook EW, 3rd. Spontaneous clearance of Chlamydia trachomatis infection in untreated patients. Sex Transm Dis. 1997;24(4):229-35.

497. Patel H, Jeve YB, Sherman SM, Moss EL. Knowledge of human papillomavirus and the human papillomavirus vaccine in European adolescents: a systematic review. Sex Transm Infect. 2016;92(6):474-9.

498. Patton MQ. Qualitative Evaluation and Research Methods. 2nd edn: Sage, Newbury Park, California; 1990.

499. Pau AK, George JM. Antiretroviral therapy: current drugs. Infect Dis Clin North Am. 2014;28(3):371-402.

500. Paul C, Dickson N, Davis PB, Yee RL, Chetwynd J, McMillan N. Heterosexual behaviour and HIV risk in New Zealand: data from a national survey. Aust J Public Health. 1995;19(1):13-8.

501. Paul C, van Roode T, Herbison P, Dickson N. Longitudinal study of self-reported sexually transmitted infection incidence by gender and age up to age thirty-two years. Sex Transm Dis. 2009;36(2):63-9.

502. Pease E, Pease B, Pease C. Do rheumatologists think about sex? Reumatol Clin. 2013;9(4):255.

503. Pedrana A, Hellard M, Guy R, El-Hayek C, Gouillou M, Asselin J, et al. Stop the drama Downunder: a social marketing campaign increases HIV/sexually transmitted infection knowledge and testing in Australian gay men. Sex Transm Dis. 2012;39(8):651-8.

504. Petrin D, Delgaty K, Bhatt R, Garber G. Clinical and microbiological aspects of Trichomonas vaginalis. Clin Microbiol Rev. 1998;11(2):300-17. 
505. Petrosky E, Bocchini JA, Jr., Hariri S, Chesson H, Curtis CR, Saraiya M, et al. Use of 9valent human papillomavirus (HPV) vaccine: updated HPV vaccination recommendations of the advisory committee on immunization practices. MMWR Morb Mortal Wkly Rep. 2015;64(11):300-4.

506. Piercy $\mathrm{H}$. The importance of contextualisation in giving a diagnosis of genital chlamydial infection: findings from a qualitative study. J Fam Plann Reprod Health Care. 2006a;32(4):227-30.

507. Piercy H. "It feels good to be told that I'm all clear": patients' accounts of retesting following genital chlamydial infection. Sex Transm Infect. 2006b;82(4):330-3.

508. Pitts MK, Woolliscroft J, Cannon S, Johnson I, Singh G. Factors influencing delay in treatment seeking by first-time attenders at a genitourinary clinic. Int J STD AIDS. 2000;11(6):375-8.

509. Poorolajal J, Hooshmand E, Mahjub H, Esmailnasab N, Jenabi E. Survival rate of AIDS disease and mortality in HIV-infected patients: a meta-analysis. Public Health.

2016;11(6):375-8.

510. Pope JE, Krizova A, Garg AX, Thiessen-Philbrook H, Ouimet JM. Campylobacter Reactive Arthritis: A Systematic Review. Semin Arthritis Rheum. 2007;37(1):48-55.

511. Popovic M, Read-Connole E, Gallo RC. T4 positive human neoplastic cell lines susceptible to and permissive for HTLV-III. Lancet. 1984;2(8417-8418):1472-3.

512. Power R. The application of qualitative research methods to the study of sexually transmitted infections. Sex Transm Infect. 2002;78(2):87-9.

513. Preventx. NHS Screening Services (Webpage). Available at: https://www.preventx.com/nhsscreening [Accessed 04/12/16].

514. Price MJ, Ades AE, De Angelis D, Welton NJ, Macleod J, Soldan K, et al. Risk of pelvic inflammatory disease following Chlamydia trachomatis infection: analysis of prospective studies with a multistate model. Am J Epidemiol. 2013;178(3):484-92.

515. Primary HPV Screening (Webpage). National Screening Unit. Available at: https://www.nsu.govt.nz/health-professionals/national-cervical-screeningprogramme/primary-hpv-screening [Accessed 04/12/16]. 2016.

516. Psutka R, Connor J, Cousins K, Kypri K. Sexual health, risks, and experiences of New Zealand university students: findings from a national cross-sectional study. $N Z \mathrm{Med} J$ 2012;125(1361):62-73.

517. Public Health England. STI diagnoses \& rates in England by gender, 2006 - 2015. Available at: https://www.gov.uk/government/uploads/system/uploads/attachment data/file/534517/2015 Table 1 STI diagnoses rates in England by gender 2006-2015.pdf [Accessed: 15/10/16]. 2016. 
518. Read P, Fairley CK, Chow EP. Increasing trends of syphilis among men who have sex with men in high income countries. Sex Health. 2015;12(2):155-63.

519. Reagan MM, Xu H, Shih SL, Secura GM, Peipert JF. A randomized trial of home versus clinic-based sexually transmitted disease screening among men. Sex Transm Dis. 2012;39(11):842-7.

520. Reece M, Herbenick D, Schick V, Sanders SA, Dodge B, Fortenberry JD. Condom use rates in a national probability sample of males and females ages 14 to 94 in the United States. J Sex Med. 2010;7 Suppl 5:266-76.

521. Regan DG, Wilson DP, Hocking JS. Coverage is the key for effective screening of Chlamydia trachomatis in Australia. J Infect Dis. 2008;198(3):349-58.

522. Rehm J, Shield KD, Joharchi N, Shuper PA. Alcohol consumption and the intention to engage in unprotected sex: systematic review and meta-analysis of experimental studies. Addiction. 2012;107(1):51-9.

523. Reveille JD, Hirsch R, Dillon CF, Carroll MD, Weisman MH. The prevalence of HLA-B27 in the US: data from the US National Health and Nutrition Examination Survey, 2009. Arthritis Rheum. 2012;64(5):1407-11.

524. Rich E, Hook IEW, Alarcon GS, Moreland LW. Reactive arthritis in patients attending an urban sexually transmitted diseases clinic. Arthritis Rheum. 1996;39(7):1172-7.

525. Richardson D, Maple K, Perry N, Ambler E, Jurd C, Fisher M. A pilot qualitative analysis of the psychosocial factors which drive young people to decline chlamydia testing in the UK: implications for health promotion and screening. Int J STD AIDS. 2010;21(3):187-90.

526. Rietmeijer CA, Alfonsi GA, Douglas JM, Lloyd LV, Richardson DB, Judson FN. Trends in clinic visits and diagnosed Chlamydia trachomatis and Neisseria gonorrhoeae infections after the introduction of a copayment in a sexually transmitted infection clinic. Sex Transm Dis. 2005;32(4):243-6.

527. Rissel C, Heywood W, de Visser RO, Simpson JM, Grulich AE, Badcock PB, et al. First vaginal intercourse and oral sex among a representative sample of Australian adults: the Second Australian Study of Health and Relationships. Sex Health. 2014;11(5):406-15.

528. Rock EM, Ireland M, Resnick MD. To know that we know what we know: perceived knowledge and adolescent sexual risk behavior. J Pediatr Adolesc Gynecol. 2003;16(6):36976.

529. Rogstad KE, Omer RMIA. Sexually transmitted infections in children and adolescents. Medicine. 2014;42(6):302-6.

530. Ronco G, Dillner J, Elfstrom KM, Tunesi S, Snijders PJ, Arbyn M, et al. Efficacy of HPVbased screening for prevention of invasive cervical cancer: follow-up of four European randomised controlled trials. Lancet. 2014;383(9916):524-32. 
531. Rose SB, Bromhead C, Lawton BA, Zhang J, Stanley J, Baker MG. Access to chlamydia testing needed for high-risk groups: patterns of testing and detection in an urban area of New Zealand. Aust N Z J Public Health. 2012;36(4):343-50.

532. Rose SB, Lawton BA, Bromhead C, MacDonald EJ, Elley CR. Poor uptake of self-sample collection kits for Chlamydia testing outside primary care. Aust N Z J Public Health. 2010;34(5):517-20.

533. Rose SB, Lawton BA, Bromhead C, Macdonald EJ, Lund KA. Self-obtained vaginal swabs for PCR chlamydia testing: a practical alternative. Aust $N$ Z J Obstet Gynaecol. 2007;47(5):415-8.

534. Rose SB, Smith MC, Lawton BA. "If everyone does it, it's not a big deal." Young people talk about chlamydia testing. N Z Med J. 2008;121(1271):33-42.

535. Rosenstock IM. Historical Origins of the Health Belief Model. Health Educ Behav. 1974;2(4):328-35.

536. Rottingen JA, Cameron DW, Garnett GP. A systematic review of the epidemiologic interactions between classic sexually transmitted diseases and HIV: how much really is known? Sex Transm Dis. 2001;28(10):579-97.

537. Rudwaleit M, van der Heijde D, Landewe R, Akkoc N, Brandt J, Chou CT, et al. The Assessment of SpondyloArthritis International Society classification criteria for peripheral spondyloarthritis and for spondyloarthritis in general. Ann Rheum Dis. 2011;70(1):25-31.

538. Rusch M, Shoveller J, Burgess S, Stancer K, Patrick D, Tyndall M. Association of sexually transmitted disease-related stigma with sexual health care among women attending a community clinic program. Sex Transm Dis. 2008;35(6):553-7.

539. Russell WC, Crawford LV. Some characteristics of the deoxyribonucleic acid from herpes simplex virus. Virology. 1963;21:353-61.

540. Sacks-Davis R, Gold J, Aitken CK, Hellard ME. Home-based chlamydia testing of young people attending a music festival--who will pee and post? BMC Public Health. 2010;10:376.

541. Sales JM, Smearman EL, Swartzendruber A, Brown JL, Brody G, DiClemente RJ. Socioeconomic-related risk and sexually transmitted infection among African-American adolescent females. J Adolesc Health. 2014;55(5):698-704.

542. Salmond GPC, Welch M. Antibiotic resistance: adaptive evolution. The Lancet. 2008;372:S97-S103.

543. Samkange-Zeeb FN, Spallek L, Zeeb H. Awareness and knowledge of sexually transmitted diseases (STDs) among school-going adolescents in Europe: a systematic review of published literature. BMC Public Health. 2011;11:727.

544. Sandelowski M. Sample size in qualitative research. Res Nurs Health. 1995;18(2):179-83. 
545. Sanmani L, Foley E, Samraj S, Rowen D, Yadegarfar G, Patel R. Patient-initiated delay at a genitourinary medicine clinic: are there public health consequences? Sex Transm Infect. 2008;84(7):560-2.

546. Saunders JB, Aasland OG, Babor TF, de la Fuente JR, Grant M. Development of the Alcohol Use Disorders Identification Test (AUDIT): WHO Collaborative Project on Early Detection of Persons with Harmful Alcohol Consumption II. Addiction. 1993;88(6):791-804.

547. Savage EJ, Marsh K, Duffell S, Ison CA, Zaman A, Hughes G. Rapid increase in gonorrhoea and syphilis diagnoses in England in 2011. Euro Surveill. 2012;17(29).

548. Sawyer RG, Moss DJ. Sexually transmitted diseases in college men: a preliminary clinical investigation. J Am Coll Health. 1993;42(3):111-5.

549. Saxton PJ, Dickson NP, Hughes AJ, Ludlam AH. Infrequent condom use with casual partners among New Zealand gay and bisexual men. N Z Med J. 2015a;128(1426):49-61.

550. Saxton PJ, Hughes AJ, Giola M. HIV prevention today: with coordinated action, we can end transmission. N Z Med J. 2015b;128(1426):8-15.

551. Scambler G. Health-related stigma. Sociol Health Illn. 2009;31(3):441-55.

552. Schoeman SA, Stewart CM, Booth RA, Smith SD, Wilcox MH, Wilson JD. Assessment of best single sample for finding chlamydia in women with and without symptoms: a diagnostic test study. BMJ. 2012;345:e8013.

553. Schreier M. Qualitative content analysis in practice: Sage, Thousand Oaks, California; 2012.

554. Schroder KE, Carey MP, Vanable PA. Methodological challenges in research on sexual risk behavior: II. Accuracy of self-reports. Ann Behav Med. 2003;26(2):104-23.

555. Scott HM, Irvin R, Wilton L, Van Tieu H, Watson C, Magnus M, et al. Sexual Behavior and Network Characteristics and Their Association with Bacterial Sexually Transmitted Infections among Black Men Who Have Sex with Men in the United States. PLoS One. 2015;10(12):e0146025.

556. Sekirime WK, Tamale J, Lule JC, Wabwire-Mangen F. Knowledge, attitude and practice about sexually transmitted diseases among university students in Kampala. Afr Health Sci. 2001;1(1):16-22.

557. Selik RM, Mokotoff ED, Branson B, Owen SM, Whitmore S, Hall HI. Revised surveillance case definition for HIV infection - United States. Centers for Disease Control and Prevention, 2014 Apr 11. Report No.: 1057-5987 Contract No.: Rr-03.

558. Sellami H, Znazen A, Sellami A, Mnif H, Louati N, Ben Zarrouk S, et al. Molecular detection of Chlamydia trachomatis and other sexually transmitted bacteria in semen of male partners of infertile couples in Tunisia: the effect on semen parameters and spermatozoa apoptosis markers. PLoS One. 2014;9(7):e98903.

559. Selmi C, Gershwin ME. Diagnosis and classification of reactive arthritis. Autoimmun Rev. 2014;13(4-5):546-9. 
560. Senior K, Helmer J, Chenhall R, Burbank V. 'Young clean and safe?' young people's perceptions of risk from sexually transmitted infections in regional, rural and remote Australia. Cult Health Sex. 2014;16(4):453-66.

561. Senn TE, Walsh JL, Carey MP. Mediators of the Relation Between Community Violence and Sexual Risk Behavior Among Adults Attending a Public Sexually Transmitted Infection Clinic. Arch Sex Behav. 2016;45(5):1069-82.

562. Serlin M, Shafer MA, Tebb K, Gyamfi AA, Moncada J, Schachter J, et al. What sexually transmitted disease screening method does the adolescent prefer? Adolescents' attitudes toward first-void urine, self-collected vaginal swab, and pelvic examination. Arch Pediatr Adolesc Med. 2002;156(6):588-91.

563. Sharpe TT, Harrison KM, Dean HD. Summary of CDC consultation to address social determinants of health for prevention of disparities in HIV/AIDS, viral hepatitis, sexually transmitted diseases, and tuberculosis. December 9-10, 2008. Public Health Rep. 2010;125 Suppl 4:11-5.

564. Sherman SM, Nailer E, Minshall C, Coombes R, Cooper J, Redman CW. Awareness and knowledge of HPV and cervical cancer in female students: A survey (with a cautionary note). J Obstet Gynaecol. 2016;36(1):76-80.

565. Shih SL, Graseck AS, Secura GM, Peipert JF. Screening for sexually transmitted infections at home or in the clinic? Curr Opin Infect Dis. 2011;24(1):78-84.

566. Shikary T, Bernstein DI, Jin Y, Zimet GD, Rosenthal SL, Kahn JA. Epidemiology and risk factors for human papillomavirus infection in a diverse sample of low-income young women. J Clin Virol. 2009;46(2):107-11.

567. Shipitsyna E, Krasnoselskikh T, Zolotoverkhaya E, Savicheva A, Krotin P, Domeika M, et al. Sexual behaviours, knowledge and attitudes regarding safe sex, and prevalence of non-viral sexually transmitted infections among attendees of youth clinics in St. Petersburg, Russia. $J$ Eur Acad Dermatol Venereol. 2013;27(1):e75-84.

568. Shoveller JA, Knight R, Johnson J, Oliffe JL, Goldenberg S. 'Not the swab!' Young men's experiences with STI testing. Sociol Health Illn. 2010;32(1):57-73.

569. Shuper PA, Neuman M, Kanteres F, Baliunas D, Joharchi N, Rehm J. Causal considerations on alcohol and HIV/AIDS - a systematic review. Alcohol Alcohol. 2010;45(2):159-66.

570. Siettos CI, Russo L. Mathematical modeling of infectious disease dynamics. Virulence. 2013;4(4):295-306.

571. Silva MJ, Florencio GL, Gabiatti JR, Amaral RL, Eleuterio Junior J, Goncalves AK. Perinatal morbidity and mortality associated with chlamydial infection: a meta-analysis study. Braz $J$ Infect Dis. 2011;15(6):533-9.

572. Silver BJ, Guy RJ, Kaldor JM, Jamil MS, Rumbold AR. Trichomonas vaginalis as a cause of perinatal morbidity: a systematic review and meta-analysis. Sex Transm Dis. 2014;41(6):36976. 
573. Silver BJ, Guy RJ, Wand H, Ward J, Rumbold AR, Fairley CK, et al. Incidence of curable sexually transmissible infections among adolescents and young adults in remote Australian Aboriginal communities: analysis of longitudinal clinical service data. Sex Transm Infect. 2015;91(2):135-41.

574. Silverman J. Hidden agendas and how to uncover them. Medicine. 2005;33(2):27-9.

575. Simms I, Fenton KA, Ashton M, Turner KM, Crawley-Boevey EE, Gorton R, et al. The reemergence of syphilis in the United Kingdom: the new epidemic phases. Sex Transm Dis. 2005;32(4):220-6.

576. Simpson S, Clifford C, Ross K, Sefton N, Owen L, Blizzard L, et al. Sexual health literacy of the student population of the University of Tasmania: results of the RUSSL Study. Sex Health. 2015;12(3):207-16.

577. Singh A, Karrar S. The role of intracellular organisms in the pathogenesis of inflammatory arthritis. Int J Inflam. 2014;2014:158793.

578. Singh AE, Romanowski B. Syphilis: review with emphasis on clinical, epidemiologic, and some biologic features. Clin Microbiol Rev. 1999;12(2):187-209.

579. Skerlev M, Culav-Koscak I. Gonorrhea: new challenges. Clin Dermatol. 2014;32(2):275-81.

580. Slater C, Robinson AJ. Sexual health in adolescents. Clin Dermatol. 2014;32(2):189-95.

581. Smith A, Agius PA, Mitchell A, Barrett C, Pitts M. Secondary Students and Sexual Health 2008, Monograph Series No. 70 Australian Research Centre in Sex, Health \& Society, La Trobe University. Melbourne, Australia: 2009.

582. Smith AM, Rissel CE, Richters J, Grulich AE, de Visser RO. Sex in Australia: the rationale and methods of the Australian Study of Health and Relationships. Aust N Z J Public Health. 2003a;27(2):106-17.

583. Smith AMA, Rissel CE, Richters J, Grulich AE, de Visser RO. Sex in Australia: Sexual identity, sexual attraction and sexual experience among a representative sample of adults. Aust N Z J Public Health. 2003b;27(2):138-45.

584. Smith DL, Battle KE, Hay SI, Barker CM, Scott TW, McKenzie FE. Ross, macdonald, and a theory for the dynamics and control of mosquito-transmitted pathogens. PLoS Pathog. 2012;8(4):e1002588.

585. Sniehotta FF, Presseau J, Araujo-Soares V. Time to retire the theory of planned behaviour. Health Psychol Rev. 2014;8(1):1-7.

586. Snipes DJ, Benotsch EG. High-risk cocktails and high-risk sex: examining the relation between alcohol mixed with energy drink consumption, sexual behavior, and drug use in college students. Addict Behav. 2013;38(1):1418-23.

587. Song A, Richters J, Crawford J, Kippax S. HIV and sexual health knowledge and sexual experience among Australian-born and overseas-born students in Sydney. J Adolesc Health. 2005;37(3):243. 
588. Sparrow M, Lewis H, Brown P, Bromhead C, Fernando D, Maitra A. Chlamydia screening in Wellington Family Planning Association (FPA) clinics: a demonstration project. $N Z$ Med J. 2007;120(1252):U2490.

589. Spauwen LW, Niekamp AM, Hoebe CJ, Dukers-Muijrers NH. Drug use, sexual risk behaviour and sexually transmitted infections among swingers: a cross-sectional study in The Netherlands. Sex Transm Infect. 2015;91(1):31-6.

590. Spleen AM, Lengerich EJ, Camacho FT, Vanderpool RC. Health care avoidance among rural populations: results from a nationally representative survey. J Rural Health. 2014;30(1):7988 .

591. Stajano C. La reaccion frenica en ginecologica. Sem Med Mex. 1920;27:243-8.

592. Stanaway JD, Wald A, Martin ET, Gottlieb SL, Magaret AS. Case-crossover analysis of condom use and herpes simplex virus type 2 acquisition. Sex Transm Dis. 2012;39(5):388-93.

593. Starrs A. A Lancet Commission on sexual and reproductive health and rights: going beyond the Sustainable Development Goals. Lancet. 2015;386(9999):1111-2.

594. Steen R, Wi TE, Kamali A, Ndowa F. Control of sexually transmitted infections and prevention of HIV transmission: mending a fractured paradigm. Bull World Health Organ. 2009;87(11):858-65.

595. Steenbeek A, Tyndall M, Rothenberg R, Sheps S. Determinants of sexually transmitted infections among Canadian Inuit adolescent populations. Public Health Nurs. 2006;23(6):531-4.

596. Steinau M, Hariri S, Gillison ML, Broutian TR, Dunne EF, Tong ZY, et al. Prevalence of cervical and oral human papillomavirus infections among US women. $J$ Infect Dis. 2014;209(11):1739-43.

597. Stephenson J, Carder C, Copas A, Robinson A, Ridgway G, Haines A. Home screening for chlamydial genital infection: is it acceptable to young men and women? Sex Transm Infect. 2000;76(1):25-7.

598. Stewart CM, Schoeman SA, Booth RA, Smith SD, Wilcox MH, Wilson JD. Assessment of self taken swabs versus clinician taken swab cultures for diagnosing gonorrhoea in women: single centre, diagnostic accuracy study. BMJ. 2012;345:e8107.

599. Stigum H, Magnus P, Bakketeig LS. Effect of changing partnership formation rates on the spread of sexually transmitted diseases and human immunodeficiency virus. Am J Epidemiol. 1997;145(7):644-52.

600. Storey GO, Scott, DL. Arthritis associated with venereal disease in nineteenth century London. Clin Rheumatol. 1998;17(6):500-4.

601. Sudenga SL, Ingles DJ, Pierce Campbell CM, Lin H-Y, Fulp WJ, Messina JL, et al. Genital Human Papillomavirus Infection Progression to External Genital Lesions: The HIM Study. Eur Urol. 2016;69(1):166-73. 
602. Tabrizi SN, Chen S, Borg AJ, Lees MI, Fairley CK, Jackson HD, et al. Patient-administered tampon-collected genital cells in the assessment of Chlamydia trachomatis infection using polymerase chain reaction. Sex Transm Dis. 1996;23(6):494-7.

603. Tampa M, Sarbu I, Matei C, Benea V, Georgescu SR. Brief history of syphilis. J Med Life. 2014;7(1):4-10.

604. Tarkowski TA, Koumans EH, Sawyer M, Pierce A, Black CM, Papp JR, et al. Epidemiology of human papillomavirus infection and abnormal cytologic test results in an urban adolescent population. J Infect Dis. 2004;189(1):46-50.

605. Theunissen KA, Bos AE, Hoebe CJ, Kok G, Vluggen S, Crutzen R, et al. Chlamydia trachomatis testing among young people: what is the role of stigma? BMC Public Health. 2015;15:651.

606. Thi Thu H, Ziersch A, Hart G. Healthcare-seeking behaviours for sexually transmitted infections among women attending the National Institute of Dermatology and Venereology in Vietnam. Sex Transm Infect. 2007;83(5):406-10.

607. Thomas JC, Tucker MJ. The development and use of the concept of a sexually transmitted disease core. J Infect Dis. 1996;174 Suppl 2:S134-43.

608. Thomas MG, Smith AJ, Tilyard M. Rising antimicrobial resistance: a strong reason to reduce excessive antimicrobial consumption in New Zealand. N Z Med J. 2014;127(1394):72-84.

609. Thomson G, Thomson B, Inman R. Validation of a screening questionnaire for epidemic reactive arthritis (QUEST-2). Arthritis Rheum. 1993;36(9).

610. Tilson EC, Sanchez V, Ford CL, Smurzynski M, Leone PA, Fox KK, et al. Barriers to asymptomatic screening and other STD services for adolescents and young adults: focus group discussions. BMC Public Health. 2004;4:21.

611. Townes JM. Reactive arthritis after enteric infections in the United States: the problem of definition. Clin Infect Dis. 2010;50(2):247-54.

612. Townes JM, Deodhar AA, Laine ES, Smith K, Krug HE, Barkhuizen A, et al. Reactive arthritis following culture-confirmed infections with bacterial enteric pathogens in Minnesota and Oregon: a population-based study. Ann Rheum Dis. 2008;67(12):1689-96.

613. Tronstein E, Johnston C, Huang ML, Selke S, Magaret A, Warren T, et al. Genital shedding of herpes simplex virus among symptomatic and asymptomatic persons with HSV-2 infection. JAMA. 2011;305(14):1441-9.

614. Turner CF, Miller HG. Zenilman's anomaly reconsidered: fallible reports, ceteris paribus, and other hypotheses. Sex Transm Dis. 1997;24(9):522-7.

615. Turner KM, Round J, Horner P, Macleod J, Goldenberg S, Deol A, et al. An early evaluation of clinical and economic costs and benefits of implementing point of care NAAT tests for Chlamydia trachomatis and Neisseria gonorrhoea in genitourinary medicine clinics in England. Sex Transm Infect. 2014;90(2):104-11. 
616. Tyden T, Bergholm M, Hallen A, Odlind V, Olsson SE, Sjoden PO, et al. Evaluation of an STD-prevention program for Swedish university students. J Am Coll Health. 1998;47(2):705.

617. Tyden T, Bjorkelund C, Odlind V, Olsson SE, Strand A. Effects of specially tailored information on Swedish university students' sexual behavior. J Am Coll Health. 1994;43(2):75-9.

618. Unemo M, Shafer WM. Antimicrobial resistance in Neisseria gonorrhoeae in the $21 \mathrm{st}$ century: past, evolution, and future. Clin Microbiol Rev. 2014;27(3):587-613.

619. UNESCO International Technical Guidance on Sexuality Education. An evidence-informed approach for schools, teachers and health educators. Paris, France: The United Nations Educational, Scientific and Cultural Organization, 2009.

620. Vaccarella S, Franceschi S, Engholm G, Lonnberg S, Khan S, Bray F. 50 years of screening in the Nordic countries: quantifying the effects on cervical cancer incidence. Br J Cancer. 2014;111(5):965-9.

621. van den Brule AJ, Munk C, Winther JF, Kjaer SK, Jorgensen HO, Meijer CJ, et al. Prevalence and persistence of asymptomatic Chlamydia trachomatis infections in urine specimens from Danish male military recruits. Int J STD AIDS. 2002;13 Suppl 2:19-22.

622. Van der Pol B. Trichomonas vaginalis infection: the most prevalent nonviral sexually transmitted infection receives the least public health attention. Clin Infect Dis. 2007;44(1):235.

623. Van Dyck E, Ieven M, Pattyn S, Van Damme L, Laga M. Detection of Chlamydia trachomatis and Neisseria gonorrhoeae by enzyme immunoassay, culture, and three nucleic acid amplification tests. J Clin Microbiol. 2001;39(5):1751-6.

624. van Rooijen MS, Koekenbier RH, Hendriks A, de Vries HJC, van Leeuwen P, van Veen MG. Young Low-Risk Heterosexual Clients Prefer a Chlamydia Home Collection Test to a Sexually Transmitted Infection Clinic Visit in Amsterdam, the Netherlands, A CrossSectional Study. Sex Transm Dis. 2016;43(11):710-6.

625. Vaughan D, O'Connell E, Cormican M, Brugha R, Faherty C, Balfe M, et al. "Pee-in-a-Pot": acceptability and uptake of on-site chlamydia screening in a student population in the Republic of Ireland. BMC Infect Dis. 2010;10:325.

626. Venkatesh KK, Cu-Uvin S. Anatomic and hormonal changes in the female reproductive tract immune environment during the life cycle: implications for HIV/STI prevention research. Am J Reprod Immunol. 2014;71(6):495-504.

627. Verhoeven V, Bovijn K, Helder A, Peremans L, Hermann I, Van Royen P, et al. Discussing STIs: doctors are from Mars, patients from Venus. Fam Pract. 2003;20(1):11-5.

628. Vijayakumar G, Mabude Z, Smit J, Beksinska M, Lurie M. A review of female-condom effectiveness: patterns of use and impact on protected sex acts and STI incidence. Int J STD AIDS. 2006;17(10):652-9. 
629. Viswanathan M, Berkman ND. Development of the RTI item bank on risk of bias and precision of observational studies. J Clin Epidemiol. 2012;65(2):163-78.

630. Vivancos R, Abubakar I, Phillips-Howard P, Hunter PR. School-based sex education is associated with reduced risky sexual behaviour and sexually transmitted infections in young adults. Public Health. 2013;127(1):53-7.

631. Voeten HA, O'Hara H B, Kusimba J, Otido JM, Ndinya-Achola JO, Bwayo JJ, et al. Gender differences in health care-seeking behavior for sexually transmitted diseases: a populationbased study in Nairobi, Kenya. Sex Transm Dis. 2004;31(5):265-72.

632. von Elm E, Altman DG, Egger M, Pocock SJ, Gotzsche PC, Vandenbroucke JP. The Strengthening the Reporting of Observational Studies in Epidemiology (STROBE) statement: guidelines for reporting observational studies. J Clin Epidemiol. 2008;61(4):344-9.

633. Waight MT, Rahman MM, Soto P, Tran T. Sexually transmitted diseases during pregnancy in Louisiana, 2007-2009: high-risk populations and adverse newborn outcomes. J La State Med Soc. 2013;165(4):219-26.

634. Walsh A, Rourke FO, Crowley B. Molecular detection and confirmation of Neisseria gonorrhoeae in urogenital and extragenital specimens using the Abbott CT/NG RealTime assay and an in-house assay targeting the porA pseudogene. Eur J Clin Microbiol Infect Dis. 2011;30(4):561-7.

635. Ward H, Mertens TE, Thomas C. Health seeking behaviour and the control of sexually transmitted disease. Health Policy Plan. 1997;12(1):19-28.

636. Ward H, Ronn M. Contribution of sexually transmitted infections to the sexual transmission of HIV. Curr Opin HIV AIDS. 2010;5(4):305-10.

637. Ward J, McGregor S, Guy RJ, Rumbold AR, Garton L, Silver BJ, et al. STI in remote communities: improved and enhanced primary health care (STRIVE) study protocol: a cluster randomised controlled trial comparing 'usual practice' STI care to enhanced care in remote primary health care services in Australia. BMC Infect Dis. 2013;13:425.

638. Ward J, Wand H, Bryant J, Delaney-Thiele D, Worth H, Pitts M, et al. Prevalence and Correlates of a Diagnosis of Sexually Transmitted Infection Among Young Aboriginal and Torres Strait Islander People: A National Survey. Sex Transm Dis. 2016;43(3):177-84.

639. Warner L, Stone KM, Macaluso M, Buehler JW, Austin HD. Condom use and risk of gonorrhea and Chlamydia: a systematic review of design and measurement factors assessed in epidemiologic studies. Sex Transm Dis. 2006;33(1):36-51.

640. Weinhardt LS, Carey MP. Does alcohol lead to sexual risk behavior? Findings from eventlevel research. Annu Rev Sex Res. 2000;11:125-57.

641. Wellings K, Field J, Wadsworth J, Johnson AM, Anderson RM, Bradshaw SA. Sexual lifestyles under scrutiny. Nature. 1990;348(6299):276-8.

642. Welsh DP, Grello CM, Harper MS. No strings attached: the nature of casual sex in college students. J Sex Res. 2006;43(3):255-67. 
643. Westrom L, Joesoef R, Reynolds G, Hagdu A, Thompson SE. Pelvic inflammatory disease and fertility. A cohort study of 1,844 women with laparoscopically verified disease and 657 control women with normal laparoscopic results. Sex Transm Dis. 1992;19(4):185-92.

644. Wetmore CM, Manhart LE, Wasserheit JN. Randomized controlled trials of interventions to prevent sexually transmitted infections: learning from the past to plan for the future. Epidemiol Rev. 2010;32:121-36.

645. Whiley DM, Tapsall JW, Sloots TP. Nucleic acid amplification testing for Neisseria gonorrhoeae: an ongoing challenge. J Mol Diagn. 2006;8(1):3-15.

646. Whincup PH, Kaye SJ, Owen CG, Huxley R, Cook DG, Anazawa S, et al. Birth weight and risk of type 2 diabetes: a systematic review. JAMA. 2008;300(24):2886-97.

647. White RM. Unraveling the Tuskegee Study of Untreated Syphilis. Arch Intern Med. 2000;160(5):585-98.

648. Wind CM, Schim van der Loeff MF, Unemo M, Schuurman R, van Dam AP, de Vries HJ. Test of Cure for Anogenital Gonorrhoea Using Modern RNA-Based and DNA-Based Nucleic Acid Amplification Tests: A Prospective Cohort Study. Clin Infect Dis. 2016;62(11):1348-55.

649. Winscott M, Taylor M, Kenney K. Sexually transmitted diseases among American Indians in Arizona: an important public health disparity. Public Health Rep. 2010;125 Suppl 4:51-60.

650. Wira CR, Fahey JV, Rodriguez-Garcia M, Shen Z, Patel MV. Regulation of mucosal immunity in the female reproductive tract: the role of sex hormones in immune protection against sexually transmitted pathogens. Am J Reprod Immunol. 2014;72(2):236-58.

651. Wolfers M, de Zwart O, Kok G. Adolescents in The Netherlands underestimate risk for sexually transmitted infections and deny the need for sexually transmitted infection testing. AIDS Patient Care STDS. 2011;25(5):311-9.

652. Wong ML, Chan RK, Tan HH, Sen P, Chio M, Koh D. Gender differences in partner influences and barriers to condom use among heterosexual adolescents attending a public sexually transmitted infection clinic in Singapore. J Pediatr. 2013;162(3):574-80.

653. Woodbridge MR, Dowell AC, Gray L. 'He said he had been out doing the traffic': general practitioner perceptions of sexually transmitted infection and HIV testing strategies for men. J Prim Health Care. 2015;7(1):50-6.

654. World Health Organization. Born Too Soon. The Global Action Report on Preterm Birth. March of Dimes, PMNCH, Save the Children. Geneva, Switzerland: 2012a.

655. World Health Organization. Brief sexuality-related communication. Recommendations for a public health approach. Geneva, Switzerland: 2015.

656. World Health Organization. Defining sexual health: Report of a technical consultation on sexual health, 28-31 January 2002. Geneva, Switzerland: 2002.

657. World Health Organization. Draft global health sector strategies. Sexually transmitted infections, 2016-2021. Geneva, Switzerland: 2016a. 
658. World Health Organization. Ebola haemorrhagic fever in Sudan, 1976. Report of a WHO/International Study Team. Bulletin of the World Health Organization. 1978a.

659. World Health Organization. Ebola haemorrhagic fever in Zaire, 1976. Report of an International Convention. Bulletin of the World Health Organization. 1978b.

660. World Health Organization. Fact sheet $\mathrm{N}^{\circ} 110$ - Sexually transmitted infections. WHO, 2013 Contract No.: February 2015.

661. World Health Organization. Fact sheet $\mathrm{N}^{\circ} 194$ - Antimicrobial Resistance. 2011a.

662. World Health Organization. Fact sheet $\mathrm{N}^{\circ} 380$ - Human papillomavirus (HPV) and cervical cancer. WHO, $2016 \mathrm{~b}$.

663. World Health Organization. Global incidence and prevalence of selected curable sexually transmitted infections 2008. 2012b.

664. World Health Organization. Global incidence and prevalence of selected sexually transmitted infections 2008. Geneva, Switzerland: 2012c.

665. World Health Organization. Global prevalence and incidence of selected curable sexually transmitted infections: overview and estimates. Geneva, Switzerland: 2001.

666. World Health Organization. Global strategy for the prevention and control of sexually transmitted infections: 2006-2015. Breaking the chain of transmission. Geneva, Switzerland: 2007.

667. World Health Organization. Prevalence and incidence of selected sexually transmitted infections, Chlamydia trachomatis, Neisseria gonorrhoeae, syphilis, and Trichomonas vaginalis: methods and results used by WHO to generate 2005 estimates. Geneva, Switzerland: $2011 b$.

668. Wright RJ, Stringer JS. Rapid testing strategies for HIV-1 serodiagnosis in high-prevalence African settings. Am J Prev Med. 2004;27(1):42-8.

669. Wright TC, Stoler MH, Behrens CM, Sharma A, Zhang G, Wright TL. Primary cervical cancer screening with human papillomavirus: end of study results from the ATHENA study using HPV as the first-line screening test. Gynecol Oncol. 2015;136(2):189-97.

670. Xiao PL, Zhou YB, Chen Y, Yang MX, Song XX, Shi Y, et al. Association between maternal HIV infection and low birth weight and prematurity: a meta-analysis of cohort studies. BMC Pregnancy Childbirth. 2015;15:246.

671. Yeung A, Temple-Smith M, Fairley C, Hocking J. Narrative review of the barriers and facilitators to chlamydia testing in general practice. Aust J Prim Health. 2015;21(2):139-47.

672. Yorke JA, Hethcote HW, Nold A. Dynamics and control of the transmission of gonorrhea. Sex Transm Dis. 1978;5(2):51-6.

673. Yu DT. Reactive arthritis. UpToDate, 2013. 
674. Zachariah R, Nkhoma W, Harries AD, Arendt V, Chantulo A, Spielmann MP, et al. Health seeking and sexual behaviour in patients with sexually transmitted infections: the importance of traditional healers in Thyolo, Malawi. Sex Transm Infect. 2002;78(2):127-9.

675. Zakher B, Kang M. Attitudes to chlamydia screening in general practice among Australian university students: a pilot study. Sex Health. 2008;5(4):359-63.

676. Zeidler H, Amor B. The Assessment in Spondyloarthritis International Society (ASAS) classification criteria for peripheral spondyloarthritis and for spondyloarthritis in general: the spondyloarthritis concept in progress. Ann Rheum Dis. 2011;70(1):1-3.

677. Zeidler H, Hudson AP. Causality of Chlamydiae in Arthritis and Spondyloarthritis: a Plea for Increased Translational Research. Curr Rheumatol Rep. 2016;18(2):9.

678. Zhan W, Krasnoselskikh TV, Golovanov S, Kozlov AP, Abdala N. Gap between consecutive sexual partnerships and sexually transmitted infections among STI clinic patients in St Petersburg, Russia. AIDS Behav. 2012;16(2):334-9.

679. zur Hausen H. Papillomaviruses in human cancers. Proc Assoc Am Physicians. 1999;111(6):581-7. 